FELIPE S. MAFFEI

Fluid Topology optimization applied to laminar jet pumps

Dissertação apresentada à Escola Politécnica da Universidade de São Paulo para obtenção do titulo de Mestre em Ciências. 


\title{
Fluid Topology optimization applied to laminar jet pumps
}

\author{
Versão Corrigida
}

Dissertação apresentada à Escola Politécnica da Universidade de São Paulo para obtenção do titulo de Mestre em Ciências.

Área de Concentração :

Engenharia de Energia e Fluidos

Orientador:

Prof. Dr. Julio Romano Meneghini

Coorientador:

Dr. Ivan Korkischko 
Autorizo a reprodução e divulgação total ou parcial deste trabalho, por qualquer meio convencional ou eletrônico, para fins de estudo e pesquisa, desde que citada a fonte.

Este exemplar foi revisado e corrigido em relação à versão original, sob responsabilidade única do autor e com a anuência de seu orientador.

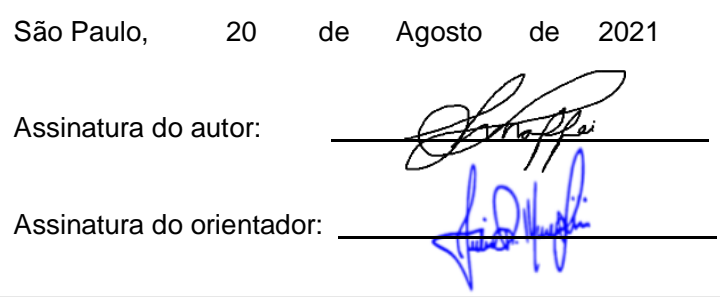

Catalogação-na-publicação

Silva Maffei, Felipe

Otimização topológica de fluidos aplicada em ejetores laminares / F. Silva Maffei -- versão corr. -- São Paulo, 2021. $124 \mathrm{p}$.

Dissertação (Mestrado) - Escola Politécnica da Universidade de São Paulo. Departamento de Engenharia Mecânica.

1.Otimização topológica 2.Ejetores 3.Mudança climática I.Universidade de São Paulo. Escola Politécnica. Departamento de Engenharia Mecânica II.t. 
Dedico esse trabalho a todos que veem na ciência um sentido para a vida. 
"If you're going to try, go all the way.

Otherwise, don't even start.

If you're going to try, go all the way.

This could mean losing girlfriends, wives, relatives, jobs and maybe your mind.

Go all the way.

It could mean not eating for 3 or 4 days.

It could mean freezing on a park bench.

It could mean jail, it could mean derision, mockery, isolation.

Isolation is the gift, all the others are a test of your endurance, of how much you really want to do it.

And you'll do it.

Despite rejection and the worst odds.

And it will be better than anything else you can imagine.

If you're going to try, go all the way.

There is no other feeling like that.

You will be alone with the gods and the nights will flame with fire.

Do it, do it, do it.

Do it.

All the way, all the way. You will ride life straight to perfect laughter, its the only good fight there is." - Charles Bukowski

"Não existe sentido em se guardar o seu conhecimento só para si. Ele sempre precisará ser repassado a outros, senão não terá qualquer valor" - Walter Edgard Maffei 


\section{ACKNOWLEDGEMENT}

I dedicate this work to my relatives Marcia Soares da Silva Maffei, Marcel Maffei and Victor Hugo Silva Maffei. Especially, I would like to thank my great-aunt Lucy Maffei Hutter. Without their support, my graduate and undergraduate studies would be unviable.

I could not forget my childhood friends. Their support and company were vital to keep on moving forward regardless of the difficulties, and my laboratory and project friends who helped me a lot several times at problems that seemed unsolvable.

I take the opportunity to thank my undergraduate professor, Dr. Edvaldo Angelo. His dedication and brilliance make up the best classes that I had the chance to attend. An exceptional professor and researcher that inspired me to follow the academic career.

I am grateful for the support of Fundação de Apoio à Universidade de São Paulo (FUSP) for the financial support. Without public funding agencies, science in Brazil is simply impracticable, and consequently, our future would be uncertain. We live in times where the value of science are put in check. However, I am convinced that science will prevail and reaffirm its value. I think that this paragraph can be summarized by the speech of Kofi Annan: "Knowledge is power. Information is liberating. Education is the premise of progress".

I would like to acknowledge Dr Julio Romano Meneghini and Dr Ivan Korkischko for accepting to be my advisor and co-advisor, respectively. I can surely say that their knowledge and advice were very useful to my development as a researcher. 


\begin{abstract}
The continuous growth of environmental awareness by the public, industrial and political sectors create conditions for scientific advancements in technologies that contribute to confront climate change. In this context, ejector (or jet pumps) could be a promising device to reduce cost, complexity and energy consumption in processes where vacuum pumps, regular pumps or regular compressors are currently employed. However, despite several scientific publications since its conception, the low efficiency of jet pumps still is a limit factor to a broad range of applications. In the field of optimization, the relatively new branch of topology optimization is a powerful methodology that makes it possible to obtain novel designs without an initial guess. In the present work, the operation condition of a conventional jet pump was simplified, the resulting boundary conditions obtained from this simplification were submitted to topology optimization. The optimization methodology used was based in the literature. However, the number of iterations was increased when compared with the reference. The first round of results (with the same objective function and restriction of base article) pointed that, in some cases, the efficiency was benefited when the final geometry resembled a conventional ejector. In the face of the first-round results and the similarity with the heat sink optimization problem, the objective functions were enhanced with a second objective (multi-objective function) to capture the ejector physics completely. The results pointed that it is possible to increase in $102 \%$ the efficiency, compared to the single objective function results, with the correct parameters. Still, an alternative approach with different objective function and constraints was tested where the results indicated that both approaches were equivalent. Although the working conditions of the simulated ejector did not reflect the usual conditions, it was possible to investigate the objective functions and to establish an optimization methodology to the jet pump field. This way, the results obtained can be used as a basis from which future works in the jet pump field can take advantage.
\end{abstract}

Keywords: Jet pump. Topology optimization. Climate change. 


\section{RESUMO}

O constante crescimento da consciência ambiental por parte da população, da industrial e das lideranças políticas criam as condições necessárias para o avanço científico em tecnologias que contribuem para confrontar o processo de aquecimento global. Nesse contexto, os ejetores podem ser um dispositivo promissor para reduzir o custo, complexidade e consumo de energia em processos onde bombas de vácuo, bombas e compressores regulares são atualmente empregados. Entretanto, apesar de inúmeras publicações científicas desde sua concepção, a baixa eficiência dos ejetores ainda é um fator limitante para uma aplicação mais ampla. No campo da otimização, o relativamente novo método da otimização topológica é uma poderosa metodologia que possibilita obter novas geometrias sem que seja feito uma estimativa inicial. Neste trabalho, o regime de operação de um ejetor convencional foi simplificado, as condições de contorno que foram obtidas a partir dessa simplificação foram submetidas à otimização topológica. A metodologia de otimização usada foi baseada em dados da literatura, entretanto, o número de iterasoes foi aumentado quando comparado com a referência. O primeiro grupo de resultados (com a função objetivo e restrição semelhantes ao artigo base) apontou que, em alguns casos, a eficiência foi beneficiada quando a geometria final era semelhante a um ejetor convencional. Em face dos resultados desse primeiro grupo de simulações e da similaridade com o problema de otimização de trocadores de calor, a função objetivo foi aprimorada com um segundo objetivo (otimização multi-objetivo) para capturar completamente a física do ejetor. Os resultados dessas simulações mostraram que é possível um ganho de até $102 \%$ na eficiência, em relação aos resultados que usaram a função objetivo simples, com a configuação correta. Ainda, foi testada uma abordagem alternativa com diferentes restrições e função objetivo onde os resultados apontaram a equivalencia entre as duas abordagens. Apesar das condições de trabalho do ejetor simulado não refletirem as condições usuais, foi possível investigar o comportamento das funções objetivo e estabelecer uma metodologia de otimização para o campo dos ejetores. Dessa forma, os resultados obtidos podem ser usados como base para trabalhos futuros na área dos ejetores.

Palavras-chave: Ejetor. Otimização topológica. Mudança climática. 


\section{LIST OF FIGURES}

1 Schematic representation of a momentum pump. . . . . . . . . . . . . 3

2 Schematic representation of jet pumps applied in district heat systems. . . . . . 3

3 Comparison of water injection mode. 1 high-pressure pump, 2 throttle valve, 3 jet pump and 4 injected well. . . . . . . . . . . . . . . . . . 4

4 Schematic representation of ejector being used as a compressor in refrigerant cycle. ............................. 5

5 Schematic representation of ejector being used drying system. . . . . . . . . 5

6 A typical jet pump geometry with its main sections and parts. The drawing is out of scale. . . . . . . . . . . . . . . . . . 7

7 Schematic representation of the experimental rig used by Winoto, Li \& Shah

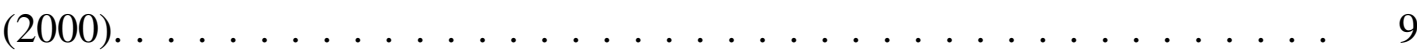

8 Pressure efficiency as a function of the flow ratio. . . . . . . . . . . . . 10

9 Pressure efficiency as a function of the flow ratio. . . . . . . . . . . . . . 10

10 The three major groups of optimization techniques. . . . . . . . . . . 12

11 Schematic representation of the dimension used in the literature review. . . . . 15

12 Schematic representation of the different types of nozzles tested in the literature. 17

13 Schematic representation of jet pump angles. . . . . . . . . . . . . 18

14 (A) Sketch of an inertia driven dosing unit. Left boundary is primary inflow. Right boundary is outlet. The secondary fluid enters from the lower port driven by the low static pressure inside the domain. (B) Result of the topology optimization conducted by Andreasen (2017). . . . . . . . . . . . . . . . . 20

15 Schematic representation of the . . . . . . . . . . . . . . 21

16 Infinitesimal element used to the derivation with the flow variables stored at the

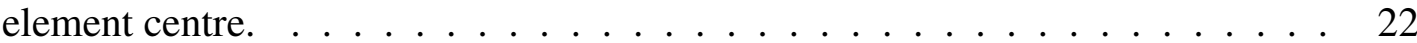


17 Mass flow at the boundaries of the domain. . . . . . . . . . . 23

18 Forces acting on the boundaries of the element. . . . . . . . . . . 25

19 Infinitesimal domain moving with the stream where the property $\varphi$ is analyzed. 27

20 First geometry with 2 D planar domain. . . . . . . . . . . . . . 37

21 Second geometry with 2D axisymmetric domain with radial secondary inlet (RSI). 39

22 Third geometry with 2D axisymmetric domain with axial secondary inlet (ASI). 40

23 Colored map of the element size distribution on the geometry $1 . \ldots 41$

24 Final solid distribution for 50, 200, 400 and 600 iteration in each step. . . . . . 49

25 Flowchart of the topology optimization routine. . . . . . . . . . . . . 50

26 Computational time for each optimization iteration as function of the number of computational nodes. . . . . . . . . . . . . . . . 51

27 Comparison of the pressure field between (A) the results using the present software configuration with the same number of optimization iterations used in the base article, (B) the results of Andreasen (2017) and (C) the result using the number of optimization iteration described in the methodology section. . . . . 53

28 Comparison of the velocity field between (A) the results using the present software configuration with the same number of optimization iterations used in the base article (B) the results of Andreasen (2017) and (C) the result using the number of optimization iterations described in the methodology section. . . . . 54

29 Plot of the momentum integral over the optimization domain and geometric parameter $r_{p} / r_{t h}$ as function of parameter $\sigma$ with Reynolds equal to $1 . \ldots 56$

30 Plot of the momentum integral over the optimization domain and geometric parameter $r_{p} / r_{t h}$ as function of parameter $\sigma$ with Reynolds equal to $1 . \quad \ldots$. . . 57

31 Flow rate $M$ and average static pressure at secondary inlet as functions of the parameter $\sigma$ for axial symmetric domain with radial secondary inlet. The values of $M$ and $C_{p}$ for $\operatorname{Re} 10$ and 30 are overlapping. . . . . . . . . . 58 
32 Flow rate $M$ and average static pressure at secondary inlet as a function of the parameter $\sigma$ for axial symmetric domain with axial secondary inlet. The values are overlapping. . . . . . . . . . . . . . . . . .

33 Comparison between the $J_{2}$ value of the filtered and the optimized domain that used the density approach to the radial and axial secondary inlet. . . . . . . . . 62

34 The efficiency $(\eta)$ and pressure efficiency $(N)$ as a function of parameter $\sigma$ for each Reynolds number simulated. . . . . . . . . . . . . . . . . . 64

35 Optimized geometry obtained for Reynolds equal to 1 and $\sigma$ equal to 5 plotted with its main dimensions. . . . . . . . . . . . . . . . . . . . . 66

36 Optimized geometry obtained for Reynolds equal to 10 and $\sigma$ equal to 5 plotted with its main dimensions. . . . . . . . . . . . . . . . . . . . . . 66

37 Optimized geometry obtained for Reynolds equal to 30 and $\sigma$ equal to 5 plotted with its main dimensions and, in red, reference lines. . . . . . . . . . . . 67

38 Plot of the normalized static pressure $\left(p_{i} *\right)$ measured along the non dimensional probe line 1 and $2\left(z_{i} *\right)$ for Reynolds number equal to 30 and $\sigma$ equal to 5,10 and 30. The reference static pressure to $p_{1} *$ normalization was $26.45 \mathrm{~Pa}$ and to $p_{2} *$ was $11.63 \mathrm{~Pa} \ldots \ldots \ldots \ldots \ldots \ldots$

39 Plot of the metrics $M, N$ and $\eta$ as function of the weight $w \ldots \ldots \ldots$

40 Geometric comparison between the two approaches. . . . . . . . . . . 75

41 Comparison between the stream lines (black seeded from the primary inlet, red from the secondary inlet and blue at the recirculation zone) obtained by the two

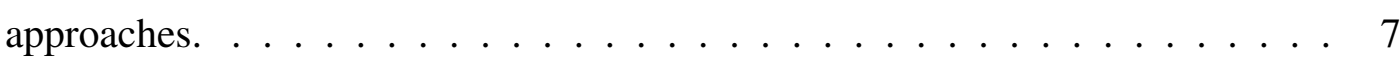

42 Comparison between the velocity field obtained by the two approaches. At the upper part, the volume constraint and, at the bottom, the pressure constraint. . . 76

43 Comparison between the pressure field obtained by the two approaches. At the upper part, the volume constraint and, at the bottom, the pressure constraint. . . 77

44 Contours of the resulting geometry using the second geometry, Reynolds equal to 1 and $\sigma$ equal to 5 . The blue and black lines matches. 
45 Contours of the resulting geometry using the second geometry, Reynolds equal to 1 and $\sigma$ equal to 10 . The blue and black lines matches. . . . . . . . . . 88

46 Contours of the resulting geometry using the second geometry, Reynolds equal to 1 and $\sigma$ equal to 30 . The blue and black lines matches. . . . . . . . . . 88

47 Contours of the resulting geometry using the second geometry, Reynolds equal to 10 and $\sigma$ equal to 5 . The blue, black and red lines matches.

48 Contours of the resulting geometry using the second geometry, Reynolds equal to 10 and $\sigma$ equal to 10 . The blue, black and red lines matches. . . . . . . .

49 Contours of the resulting geometry using the second geometry, Reynolds equal to 10 and $\sigma$ equal to 30 . The blue, black and red lines matches. . . . . . . . .

50 Contours of the resulting geometry using the second geometry, Reynolds equal to 30 and $\sigma$ equal to 5 . The blue, black and red lines matches.

51 Contours of the resulting geometry using the second geometry, Reynolds equal to 30 and $\sigma$ equal to 10 . The blue, black and red lines matches. . . . . . . . . . 90

52 Contours of the resulting geometry using the second geometry, Reynolds equal to 30 and $\sigma$ equal to 30 . The blue, black and red lines matches.

53 Contours of the resulting geometry using the third geometry, Reynolds equal to 1 and $\sigma$ equal to 5 . The blue and black lines matches. The red line slight diverges at the base of external wall . . . . . . . . . . . . . . . 90

54 Contours of the resulting geometry using the third geometry, Reynolds equal to 1 and $\sigma$ equal to 10 . The blue and black lines matches. The red line slight diverges at the base of external wall. . . . . . . . . . . . . . .

55 Contours of the resulting geometry using the third geometry, Reynolds equal to 1 and $\sigma$ equal to 5 . The blue and black lines matches. The red line slight diverges at the base of external wall. . . . . . . . . . . . . .

56 Contours of the resulting geometry using the third geometry, Reynolds equal to 10 and $\sigma$ equal to 5 . The blue, black and red lines matches. . . . . . . . . . 91

57 Contours of the resulting geometry using the third geometry, Reynolds equal to 10 and $\sigma$ equal to 10 . The blue, black and red lines matches. . . . . . . . . 
58 Contours of the resulting geometry using the third geometry, Reynolds equal to 10 and $\sigma$ equal to 30 . The blue, black and red lines matches. . . . . . . . . . 92

59 Contours of the resulting geometry using the third geometry, Reynolds equal to 30 and $\sigma$ equal to 5. The blue, black and red lines matches. . . . . . . . . . . 92

60 Contours of the resulting geometry using the third geometry, Reynolds equal to 30 and $\sigma$ equal to 10 . The blue, black and red lines matches. . . . . . . . . . 93

61 Contours of the resulting geometry using the third geometry, Reynolds equal to 30 and $\sigma$ equal to 30. The blue, black and red lines matches. . . . . . . . . 93

62 (a) Streamlines seed from the inlet (black) and secondary inlet (red) with the solid distribution (black structures), (b) velocity colour map and (c) static pressure contours with the solid distribution (black structures). Results obtained with Reynolds number equal to 1 and $\sigma$ equal to 5. . . . . . . . . . . . . . . . 9

63 (a) Stream lines seed from the inlet (black) and secondary inlet (red) with the solid distribution (black structures), (b) velocity colour map and (c) static pressure contours with the solid distribution (black structures). Results obtained with Reynolds number equal to 1 and $\sigma$ equal to $10 \ldots \ldots \ldots$. . . . . .

64 (a) Stream lines seed from the inlet (black) and secondary inlet (red) with the solid distribution (black structures), (b) velocity colour map and (c) static pressure contours with the solid distribution (black structures). Results obtained with Reynolds number equal to 1 and $\sigma$ equal to $30 \ldots \ldots$. . . . . . . . . . . 96

65 (a) Stream lines seed from the inlet (black) and secondary inlet (red) with the solid distribution (black structures), (b) velocity colour map and (c) static pressure contours with the solid distribution (black structures). Results obtained with Reynolds number equal to 10 and $\sigma$ equal to $5 \ldots \ldots$. . . . . . . . . . 97

66 (a) Stream lines seed from the inlet (black) and secondary inlet (red) with the solid distribution (black structures), (b) velocity colour map and (c) static pressure contours with the solid distribution (black structures). Results obtained with Reynolds number equal to 10 and $\sigma$ equal to 10 . . . . . . . . . . . . . . 98 
67 (a) Stream lines seed from the inlet (black) and secondary inlet (red) with the solid distribution (black structures), (b) velocity colour map and (c) static pressure contours with the solid distribution (black structures). Results obtained with Reynolds number equal to 10 and $\sigma$ equal to $30 . \ldots$. . . . . . . . . . 999

68 (a) Stream lines seed from the inlet (black), secondary inlet (red) and recirculation zones (blue) with the solid distribution (black structures), (b) velocity colour map and (c) static pressure contours with the solid distribution (black structures). Results obtained with Reynolds number equal to 30 and $\sigma$ equal to 5.100

69 (a) Stream lines seed from the inlet (black) and secondary inlet (red) and recirculation zones (blue) with the solid distribution (black structures), (b) velocity colour map and (c) static pressure contours with the solid distribution (black structures). Results obtained with Reynolds number equal to 30 and $\sigma$ equal to 10

70 (a) Stream lines seed from the inlet (black) and secondary inlet (red) and recirculation zones (blue) with the solid distribution (black structures), (b) velocity colour map and (c) static pressure contours with the solid distribution (black structures). Results obtained with Reynolds number equal to 30 and $\sigma$ equal to

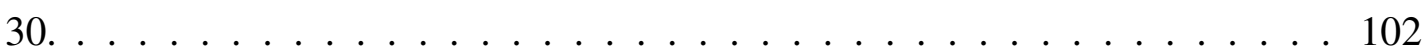

71 (a) Stream lines seed from the inlet (black) and secondary inlet (red) with the solid distribution (black structures), (b) velocity colour map and (c) static pressure contours with the solid distribution (black structures). Results obtained with Reynolds number equal to 30, $\sigma$ equal to 30 and $w$ equal to 0.0 . . . . . . . 103

72 (a) Stream lines seed from the inlet (black) and secondary inlet (red) with the solid distribution (black structures), (b) velocity colour map and (c) static pressure contours with the solid distribution (black structures). Results obtained with Reynolds number equal to 30, $\sigma$ equal to 30 and $w$ equal to 0.1 . . . . . . 104

73 (a) Stream lines seed from the inlet (black) and secondary inlet (red) with the solid distribution (black structures), (b) velocity colour map and (c) static pressure contours with the solid distribution (black structures). Results obtained with Reynolds number equal to 30, $\sigma$ equal to 30 and $w$ equal to 0.2 . . . . . . 105 
74 (a) Stream lines seed from the inlet (black) and secondary inlet (red) with the solid distribution (black structures), (b) velocity colour map and (c) static pressure contours with the solid distribution (black structures). Results obtained with Reynolds number equal to 30, $\sigma$ equal to 30 and $w$ equal to 0.3 . . . . . . 106

75 (a) Stream lines seed from the inlet (black) and secondary inlet (red) with the solid distribution (black structures), (b) velocity colour map and (c) static pressure contours with the solid distribution (black structures). Results obtained with Reynolds number equal to $30, \sigma$ equal to 30 and $w$ equal to 0.4 . . . . . . 107

76 (a) Stream lines seed from the inlet (black) and secondary inlet (red) with the solid distribution (black structures), (b) velocity colour map and (c) static pressure contours with the solid distribution (black structures). Results obtained with Reynolds number equal to $30, \sigma$ equal to 30 and $w$ equal to 0.5 . . . . . . 108

77 (a) Stream lines seed from the inlet (black) and secondary inlet (red) with the solid distribution (black structures), (b) velocity colour map and (c) static pressure contours with the solid distribution (black structures). Results obtained with Reynolds number equal to $30, \sigma$ equal to 30 and $w$ equal to 0.6 . . . . . . . 109

78 (a) Stream lines seed from the inlet (black) and secondary inlet (red) with the solid distribution (black structures), (b) velocity colour map and (c) static pressure contours with the solid distribution (black structures). Results obtained with Reynolds number equal to $30, \sigma$ equal to 30 and $w$ equal to 0.7 . . . . . . 110

79 (a) Stream lines seed from the inlet (black) and secondary inlet (red) with the solid distribution (black structures), (b) velocity colour map and (c) static pressure contours with the solid distribution (black structures). Results obtained with Reynolds number equal to $30, \sigma$ equal to 30 and $w$ equal to 0.8 . . . . . 111

80 (a) Stream lines seed from the inlet (black) and secondary inlet (red) with the solid distribution (black structures), (b) velocity colour map and (c) static pressure contours with the solid distribution (black structures). Results obtained with Reynolds number equal to $30, \sigma$ equal to 30 and $w$ equal to 0.9 . . . . . . 112 
81 (a) Stream lines seed from the inlet (black) and secondary inlet (red) with the solid distribution (black structures), (b) velocity colour map and (c) static pressure contours with the solid distribution (black structures). Results obtained with Reynolds number equal to 30, $\sigma$ equal to 30 and $w$ equal to 0.925 . . . . 113

82 (a) Stream lines seed from the inlet (black) and secondary inlet (red) with the solid distribution (black structures), (b) velocity colour map and (c) static pressure contours with the solid distribution (black structures). Results obtained with Reynolds number equal to $30, \sigma$ equal to 30 and $w$ equal to 0.95 . . . . . . 114

83 (a) Stream lines seed from the inlet (black) and secondary inlet (red) with the solid distribution (black structures), (b) velocity colour map and (c) static pressure contours with the solid distribution (black structures). Results obtained with Reynolds number equal to $30, \sigma$ equal to 30 and $w$ equal to $0.975 . \quad \ldots .115$

84 (a) Stream lines seed from the inlet (black) and secondary inlet (red) with the solid distribution (black structures), (b) velocity colour map and (c) static pressure contours with the solid distribution (black structures). Results obtained with Reynolds number equal to 30, $\sigma$ equal to 30 and $w$ equal to 0.99 . . . . . . 116

85 (a) Stream lines seed from the inlet (black) and secondary inlet (red) with the solid distribution (black structures), (b) velocity colour map and (c) static pressure contours with the solid distribution (black structures). Results obtained with Reynolds number equal to 30, $\sigma$ equal to 30 and $w$ equal to 0.995 . . . . . 11

86 (a) Stream lines seed from the inlet (black) and secondary inlet (red) with the solid distribution (black structures), (b) velocity colour map and (c) static pressure contours with the solid distribution (black structures). Results obtained with Reynolds number equal to $30, \sigma$ equal to 30 and $w$ equal to 1.0 . . . . . . 118

87 Plot of quantity $M_{u}$ in function of ratio values for geometry 1 (black), geometry 2 (green) and geometry 3 (blue). The dashed indicates the ratio of 10 used in the course mesh. . . . . . . . . . . . . . . . . . . . . . 119

88 Plot of external contours of solid distribution taken from results obtained with the fine mesh and course mesh for geometry 1 . The Reynolds number was set up equal to 20 and $\sigma$ equal to $10 \ldots \ldots \ldots \ldots$. . . . . . . . . . . 120 
89 Plot of external contours of solid distribution taken from results obtained with the fine mesh and course mesh for geometry 2 . The Reynolds number was set up equal to 30 and $\sigma$ equal to $30 \ldots \ldots$. . . . . . . . . . . . . . 120

90 Plot of external contours of solid distribution taken from results obtained with the fine mesh and course mesh for geometry 3. The Reynolds number was set up equal to 30 and $\sigma$ equal to $30 \ldots \ldots \ldots \ldots \ldots$. . . . . . . . . . . . . . .

91 Comparison between the solid and void distribution of the original and extended geometries. . . . . . . . . . . . . . . . . 123

92 Comparison between the velocity field of the original and extended geometries. 123

93 Comparison between the pressure field of the original and extended geometries. 124

94 Comparison between the streamlines of the original and extended geometries. . 124 


\section{LIST OF TABLES}

1 Numerical methods and its respective weights. . . . . . . . . . . . . . 30

$2 \quad \kappa$ variable values with respect to the geometry for $J_{2}$ objective function. . . . . 43

3 Relative difference between the values of $C p$ evaluated from the optimized domains obtained by each objective function to the radial secondary inlet. The values of domain calculated with $J_{3}$ was set up as reference All the used data is in percentage. ..................... 59

4 Relative difference between the values of $M$ evaluated from the optimized domains obtained by each objective function to the radial secondary inlet. The values of domain calculated with $J_{3}$ was set up as reference All the used data is

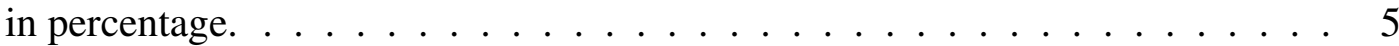

5 Relative difference between the values of $C p$ evaluated from the optimized domains obtained by each objective function to the axial secondary inlet. The values of domain calculated with $J_{3}$ was set up as reference All the used data is in percentage. . . . . . . . . . . . . . . .

6 Table of relative difference between the values of $M$ evaluated from the optimized domains obtained by each objective function to the axial secondary inlet. The values of the domain calculated with $J_{3}$ was set up as a reference. All the used data was in percentage.

7 Table of relative error between the quantities measured in the verification and density optimization simulations of $J_{2}$ objective function for radial secondary inlet and axial secondary inlet geometries. All the used data is in percentage. .

8 Table of relative difference between two consecutive total pressure efficiency $N$ values. All used data are in percentage. . . . . . . . . . . . . . . . . . . . . . 67

9 Geometrical trend during the parameter exploration. . . . . . . . . . . . . . 69

10 Table of relative difference between two consecutive $N$ values. All used data are in percentage. . . . . . . . . . . . . . . . . 12 


\section{LIST OF ABBREVIATIONS}

AM Adjoint method

ASI Axial secondary inlet

AV Average value

BWR Boiler water reactors

CFD Computational Fuid Dynamics

DD Direct Differentiation

FD Finite difference

FDM Finite difference method

FEM Finite element method

FVM Finite volume method

GBM Gradient based methods

GCMMA Globally converged method of moving asymptotes

GLS Galerkin/least squares

MMA Method of moving asymptotes

MOF Multi-objective function

MUMPS Multifrontal massively parallel sparse direct solver

NSE Navier-Stokes equations

RCGI Research Center for Gas Innovation

RSI Radial secondary inlet

SOF Single objective function

USP Universidade de São Paulo 


\section{CONTENTS}

1 Introduction 1

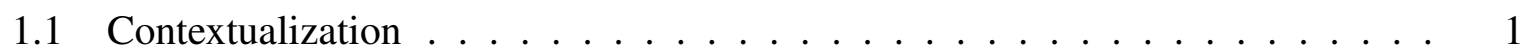

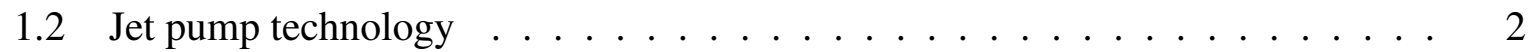

1.3 Jet pump efficiency . . . . . . . . . . . . . . . . . . 8

1.4 Topology optimization $\ldots \ldots \ldots \ldots \ldots \ldots \ldots$

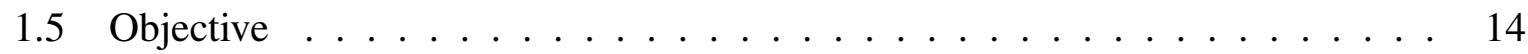

2 Literature review $\quad 15$

2.1 Search considerations . . . . . . . . . . . . . . . . . . 15

2.2 Literature review - jet pumps performance enhancing . . . . . . . . . 15

3 Methodology 22

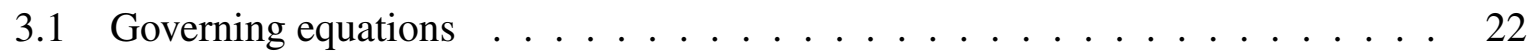

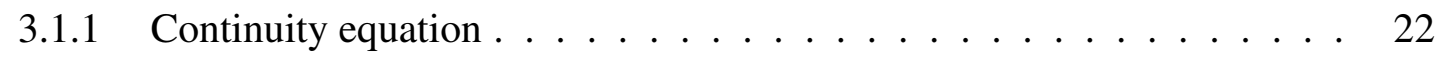

3.1.2 Momentum equation . . . . . . . . . . . . . . . . 24

3.2 Numerical approach . . . . . . . . . . . . . . . . . . 28

3.2.1 Finite element method . . . . . . . . . . . . . . 28

3.2.2 Numerical solution of the Navier-Stokes equation . . . . . . . . . . . 30

3.3 Topology optimization - The density approach . . . . . . . . . . . 33

3.4 Solver - Fluid flow problem . . . . . . . . . . . . . . . . 35

4 Geometry and mesh $\quad 37$

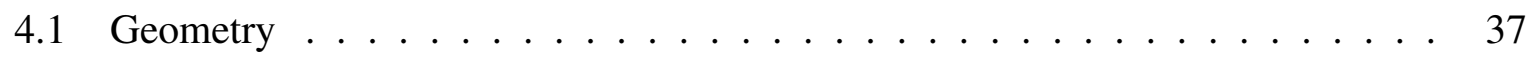

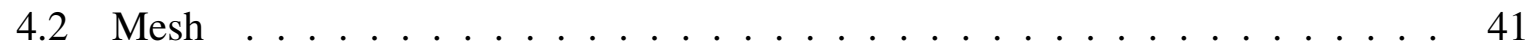


5 Optimization problem $\quad 42$

5.1 Sensitivity evaluation . . . . . . . . . . . . . . . 43

5.2 Optimization algorithm $\ldots \ldots \ldots \ldots \ldots \ldots$

5.3 Optimization strategy $\ldots \ldots \ldots \ldots \ldots \ldots$

6 Results $\quad 51$

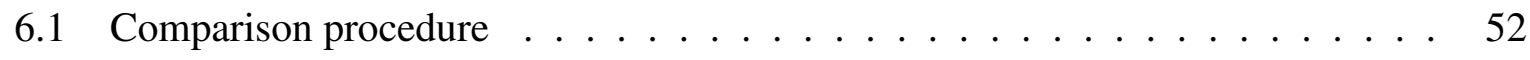

6.2 Objective functions analyses and comparison $\ldots \ldots \ldots 5$

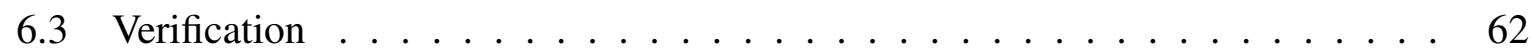

6.4 Flow analysis . . . . . . . . . . . . . . . . . 64

6.5 Multi-objective approach . . . . . . . . . . . . . . . . 70

6.5 .1 Multi-objective results . . . . . . . . . . . . . 70

6.6 Alternative approach - single objective function $\ldots \ldots . \ldots 73$

6.6.1 Results of the alternative approach (single objective function) $\ldots . . .74$

$\begin{array}{lll}7 & \text { Conclusion } & 78\end{array}$

$\begin{array}{lr}\text { References } & 80\end{array}$

$\begin{array}{ll}\text { Appendix A - Geometry comparison } & 87\end{array}$

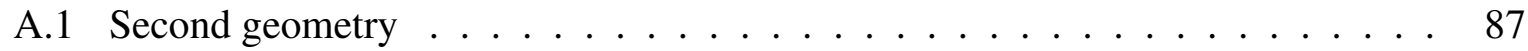

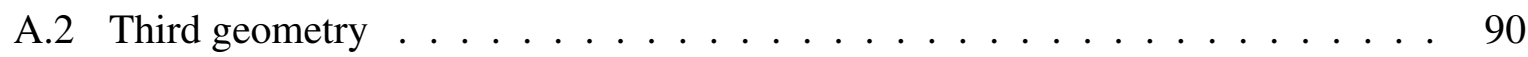

Appendix B - Flow field $\quad 94$

$\begin{array}{ll}\text { Appendix C - Flow field } & 103\end{array}$

Appendix D - Mesh convergence $\quad 119$ 


\section{INTRODUCTION}

\subsection{Contextualization}

Unrestricted human activities around the globe are continuously affecting the environment (YIN et al., 2020). Around $97 \%$ of the scientific community agrees that the rise in the average global temperature, beyond its natural heating, is clear evidence of these activities (COOK et al., 2013). Consequently, events like droughts, floods, and heatwaves become more severe and frequent (IPCC, 2014), resulting in several damages, not fully understood, in all countries' social and economic sectors. For this reason, environmental awareness by public, industrial and political sectors are in constant growth, as well as the scientific community concerned about it. All this attention drives a number of innovations in techniques, devices and methodologies to reduce the consumption of natural resources and the consequent human impact in nature.

The time scale related to the climate changes in scientific studies are around fifty to one hundred years (URBAN et al., 2016). Such scale of time is higher than those felt by the anthropogenic systems, making complex the projection of the human activity in such time interval. Therefore, besides the rise of the negation of the phenomena, improvement in human activity could not happen at a sufficient rate. An example of it can be seen at the coronavirus outbreak that, despite warnings of the scientific community for the human contact with wild animals (FAN et al., 2019; JONES et al., 2008), the practice continues, generating the favourable scenario for the rising of a new virus. Once the outbreak was established, the countries needed to adopt serious measures to effectively contain the virus (ATALAN, 2020; HYAFIL; MORIÑA, 2020). The direct consequences of this were damages to several sectors of the affected countries (PAKENHAM et al., 2020; KANITKAR, 2020) and a slow recovery of the normality. Similarly, climate change can devolve into the same behaviour. It comes slowly, but once its consequences are no longer negligible, a considerable endeavour will be needed to control it. Furthermore, the inertia associated with this event can worsen the situation even after all this endeavour (CHAPMAN, 2007). Comparably to the Sars-CoV-2 outbreak, the endeavour and cost could be more significant than if actions were taken beforehand (DOBSON et al., 2020). In this context, it is vital to investigate all the technology that can save energy or resources and its implementation viability assessed. Devices like momentum pumps (also called jet pumps, ejector or injectors) could be a promising technology to this end, reducing the energy consump- 
tion of systems (e.g. district heat systems or oil production) or reducing the operational cost of technologies that mitigate the human impact on nature.

The latter is the scope of project number 38 of the Research Center for Gas Innovation (RCGI). The center has the objective of being a world reference center for fundamental and applied research related to natural gas, biogas, hydrogen and $\mathrm{CO} 2$ management, transport and storage, and its contributions to global sustainability in the 21 st century. Specifically, the quoted project study the efficiency argumentation of an ejector that uses water as motive fluid and CO2 as secondary fluid to be applied in the compression stage of the Carbon, Capture and Storage (CCS) technology. Besides the operational cost reduction, ejectors can reduce the leakage of $\mathrm{CO} 2$ in CCS systems, once the labyrinth seals used in (inherent characteristic turbo machinery devices that use rotors) can not completely block the flow.

\subsection{Jet pump technology}

Jet pumps, ejector and injector are names that can be assigned to the momentum pump (ENGINEERING SCIENCES DATA UNIT, 1985). However, the names are usually assigned depending on the field, and fluid of the application (MEHTA, 1968). For example, the literature often reported ejectors as devices that work with compressible fluids (RUANGTRAKOON; THONGTIP, 2020; DONG et al., 2020) whereas jet pumps are often related to the application of incompressible fluids (SILVESTER, 1960; MUELLER, 1964). In this work, the devices that work with incompressible fluids will receive the name jet pumps or ejectors. The next paragraph the was written based on the Figure 1.

They use the Venturi effect promoted by the jet (created due to the nozzle shape) to suction a second fluid (blue region). The momentum of the motive fluid (red region) is then gradually transferred to the suctioned fluid in the throat. The diffuser decelerated the flow at the end of the device, recovering the static pressure. At the jet pump outlet, the mixed fluid (yellow region) has a higher pressure than the suctioned fluid and lower than the motive fluid. In other words, the secondary fluid is pumped.

As depicted in Figure 1, this hydraulic device has four principal parts: nozzle, suction chamber, throat and diffuser (TROUT; KEMPER; AUST, 1980). Its structural simplicity gives rise to attractive advantages like no moving parts, absence of seals, ease to install, low maintenance cost, and high reliability (SANGER, 1968). These advantages result in the widespread 
Figure 1 - Schematic representation of a momentum pump.

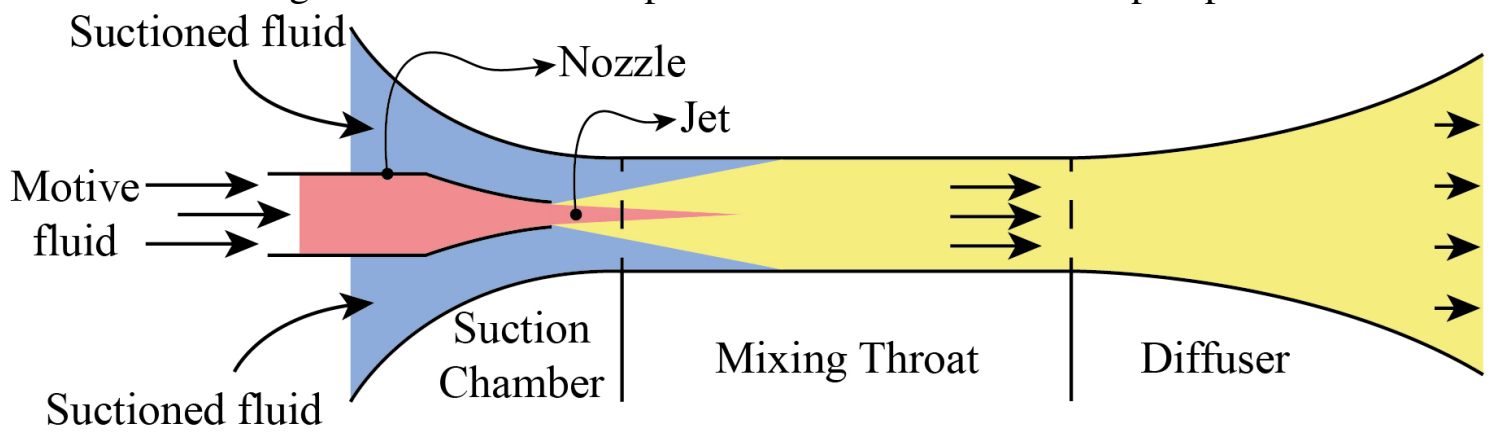

Source: Author.

use of ejectors in the industry. However, the jet pumps have low efficiency when compared to another flux machines that can replace it and narrow operational point, that is, small deviations from the operational point drastically drops the efficiency (REIS; GIORIA, 2021).

In district heat or cooling systems, the jet pumps replace the valves used to balance the hydraulic system of cooling or heating water and take advantage of the suction of the secondary fluid to reduce the flow rate in the high-pressure pipeline. This way, the global benefit has a cubic behaviour because both the flow rate of the circulation pump (see Figure 2) and the head loss in the high-pressure pipeline decrease. (WANG; WANG, 2018; LIU; ZHAN; LIA, 2017; LIU; LI; ZENG, 2017).

Figure 2 - Schematic representation of jet pumps applied in district heat systems.

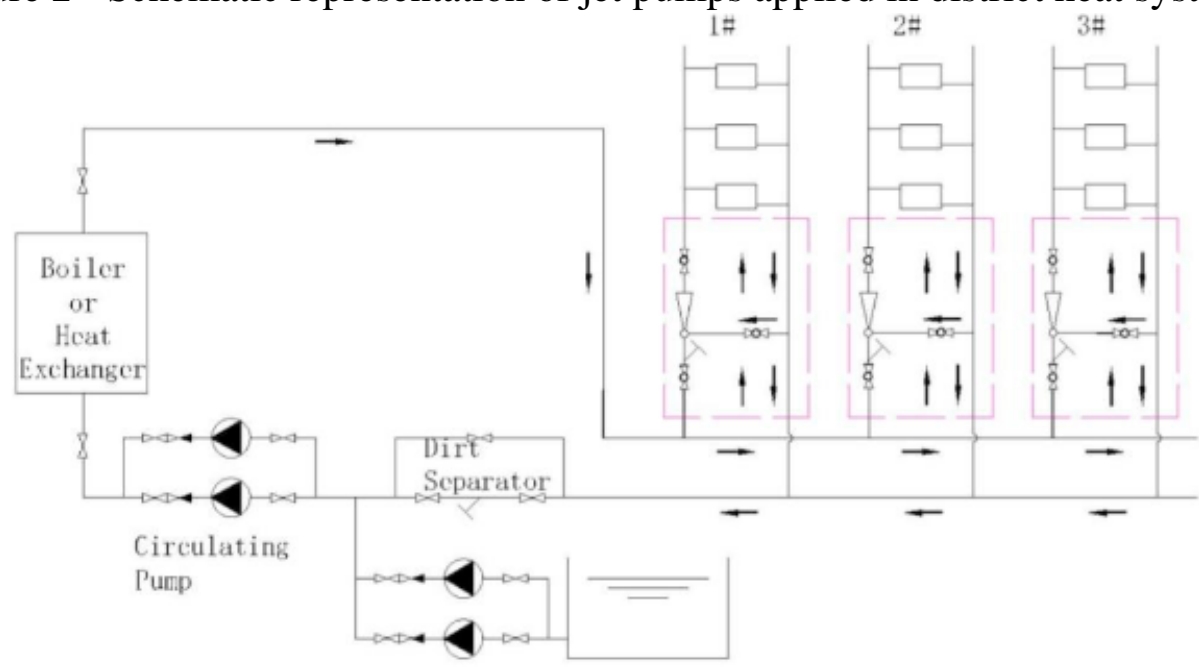

Feed water punp

Source: (LIU; ZHAN; LIA, 2017).

In the offshore oil platforms, jet pumps can replace the valves used to balance the oil wells during the application of artificial lifting technique, reducing the flow rate at the high pressure 
pumps (see Figure 3) and, hence, power consumption. Yan et al. (2018) studied this application; they concluded that the use of jet pumps could save 1.15 million $\mathrm{kW} \cdot \mathrm{h}$ in one year.

Figure 3 - Comparison of water injection mode. 1 high-pressure pump, 2 throttle valve, 3 jet pump and 4 injected well.
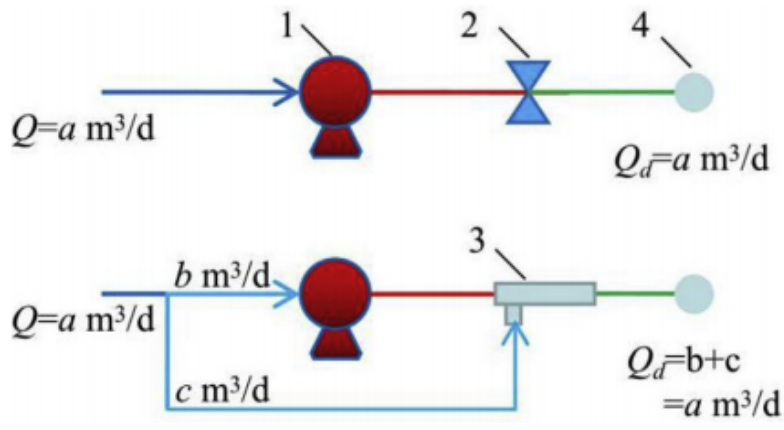

Source: (YAN et al., 2018).

In the field of pisciculture, jet pumps can be used at the critical stage of fish transportation, where, between the alternatives, the annular ejectors (an alternative design where the high pressure flow enters through the secondary inlet) can minimize the mortality rate after the transport (LONG et al., 2019; XU et al., 2019).

In most hydraulic devices, cavitation is not desirable because it can cause a reduction of their lifespan and operational problems. With jet pumps, this phenomenon is more susceptible to occur because of the characteristic high velocity and shear stress during operation; however, in some applications, the instantaneous properties caused by the collapse of the cavitation bubbles can be beneficial. For example, sewage treatment, food engineering, and chemical engineering can take advantage of this phenomena (LONG et al., 2018; WANG et al., 2018).

The application of jet pumps inside boiler water reactors (BWR) is an important unique feature of this kind of reactors, increasing its safety and reliability (THERIAULT, 2016). Even though BWR is a consolidated technology, it still is an active area of research (MANGIALARDO et al., 2014; CUAMATZI-MELENDEZ; FLORES-CUAMATZI, 2020).

Still, ejectors can be applied at deep water wells (STEPANOFF, 1957), in hydroelectric plants (SILVESTER, 1960), siphons and dredging (MUELLER, 1964).

The other variants of ejectors can work with any combination of primary fluid and secondary fluid, like: gas-liquid (CHEN et al., 2018), gas-gas (CHEN et al., 2020) and liquid-gas (ISMAGILOV; SPIRIDONOV; BELKINA, 2017), respectively. This flexibility makes possible the applications of ejectors in many other industrial sectors in place of compressors (BEN- 
CHARIF et al., 2020) (e.g. refrigeration cycle (SUN, 1999) as depicted in Figure 4), and vacuum pumps (e.g. vacuum drying method applied to solid products that are heat sensitive (SURYANTO; HAMZAH; TAUFIK, 2021) as shown in Figure 5) (EBDALE, 1978).

Figure 4 - Schematic representation of ejector being used as a compressor in refrigerant cycle.

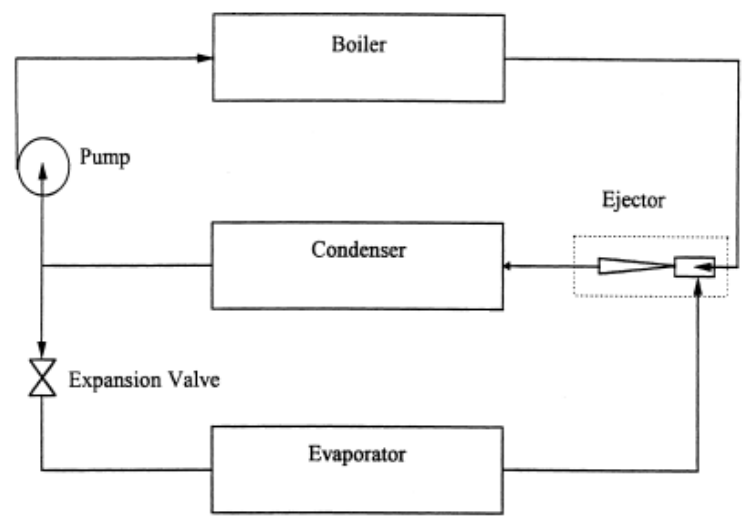

Source: (SUN, 1999).

Figure 5 - Schematic representation of ejector being used drying system.

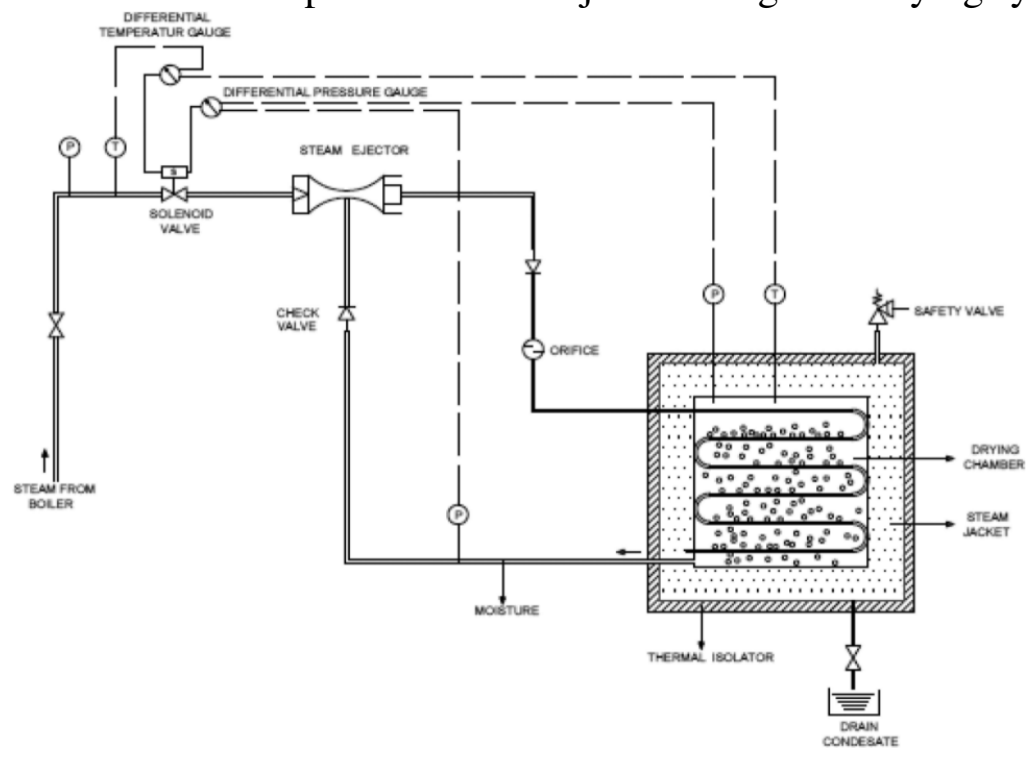

Source: (SURYANTO; HAMZAH; TAUFIK, 2021).

Among the kinds of ejectors quoted above, the liquid-liquid case are relatively easy to model, simulate, and test, so the implementation of new methodologies to enhance the ejector technology can be easier assessed in such flows. The results obtained with simpler flows could be then used as starting point (i.e. using the methodology and know-how) to ejectors with a more complex fluid flow (e.g. biphasic ejector). 
To further explaining the operation principle of jet pumps: the nozzle is responsible for converting the pressure energy of the flow fed by an external source into a high-velocity jet. The jet creates a low-pressure zone that induces the secondary liquid towards it. Starting from the nozzle tip, a high turbulent shear stress layer starts to form and transfer momentum from the jet core to the secondary liquid. As the flow moves downstream, the thickness of the layer grows until the entire jet core is mixed and, hence, the velocity profile is normalized. This process occurs mainly inside the mixing throat, and it is also responsible for recovering part of the static pressure. Finally, the flow reaches the diffuser, where it slowly decelerates, and the static pressure is recovered (SANGER, 1968). Usually, to the central jet pump working with incompressible fluids, the fluid that comes from the high-pressure source is called primary fluid or working fluid, and the fluid that is sucked is called secondary fluid or entrained fluid. One can observe that the secondary fluid has its head pressure increased whereas the primary fluid has its head pressure decreased and the outflow, an intermediate value. Figure 6 depicts a schematic representation of the device described above.

From Figure 6 it is possible to define some relations that are frequently used to describe the ejector geometry, like the proportion between the areas of outlet nozzle section $\left(A_{\text {Out }}\right)$ and throat $\left(A_{T h}\right)$ defined by the relation.

$$
b=\frac{A_{N O u t}}{A_{T h}}=\frac{\frac{\pi d_{N O u t}^{2}}{4}}{\frac{\pi d_{T h}^{2}}{4}}=\left(\frac{d_{N O u t}}{d_{T h}}\right)^{2}
$$

Proportion between the distance NXP (distance between the nozzle outlet section and throat inlet section) and the diameter of the outlet nozzle section $\left(d_{N O u t}\right)$

$$
S=\frac{N X P}{d_{\text {NOut }}}
$$

The diffuser area ratio

$$
c=\frac{A_{\text {Outlet }}}{A_{T h}}=\frac{\frac{\pi d_{\text {Outlet }}^{2}}{4}}{\frac{\pi d_{T h}^{2}}{4}}=\left(\frac{d_{\text {Outlet }}}{d_{T h}}\right)^{2}
$$

Where $d_{\text {Outlet }}$ is the outlet section diameter and $d_{T h}$ is the throat section diameter. The proportion between the throat length $L_{T h}$ and throat diameter

$$
l=\frac{L_{T h}}{d_{T h}}
$$


Figure 6 - A typical jet pump geometry with its main sections and parts. The drawing is out of scale.

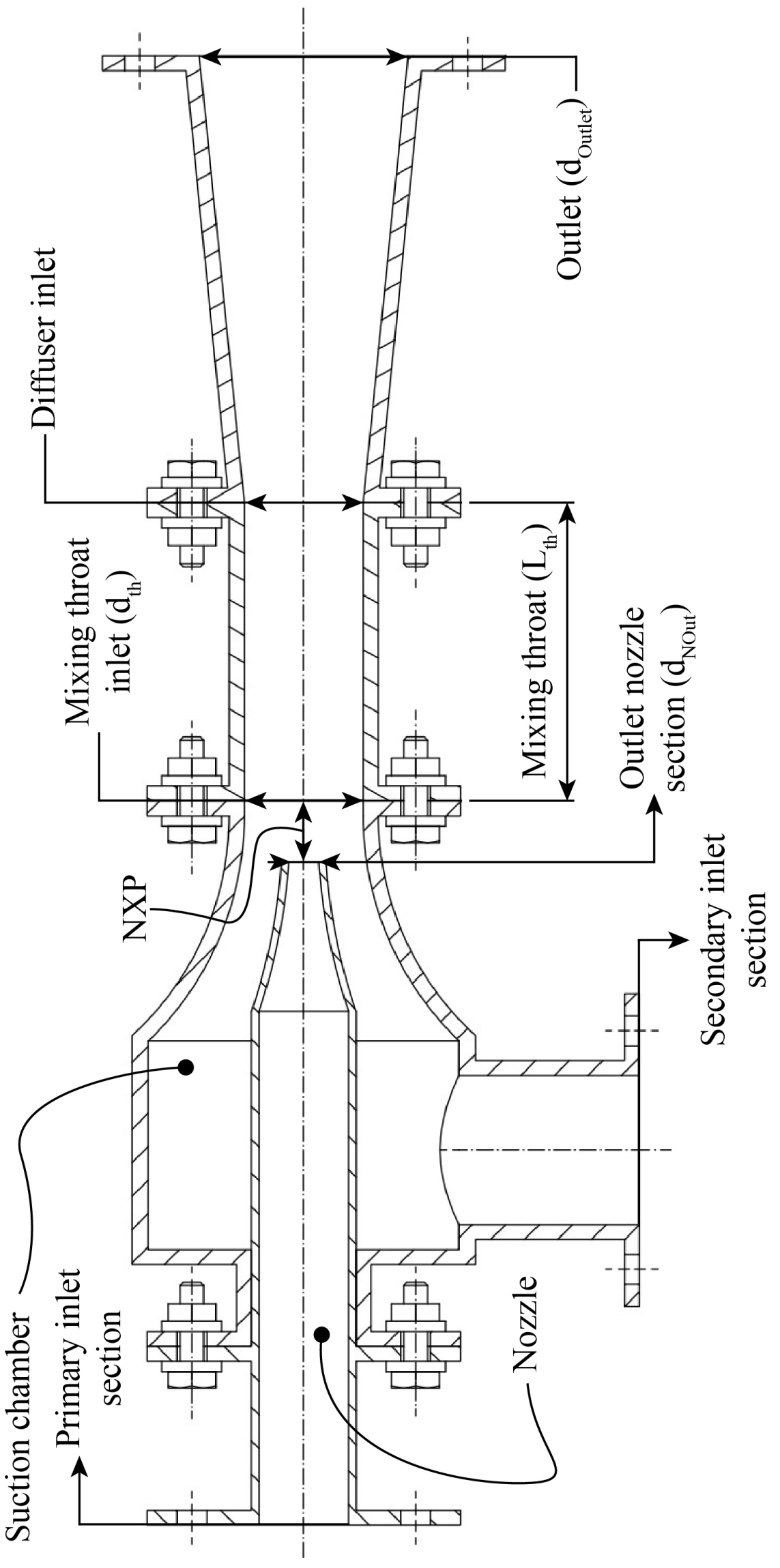

Source: Author. 
Despite the advantages and the broad field of application of ejectors, the low efficiency still an issue in the field of jet pumps (YAPICI; ALDAS, 2013; NARABAYASHI et al., 2006). Other important features with respect to the practical application of jet pumps are the narrow optimum region of operation, that is, small deviations of the designed operation point, drastically affect the efficiency (YAPICI; ALDAS, 2013) and cavitation, which has a significant influence in its operation (BROWN, 1970).

The three known ways to evaluate the ejector efficiency are (i) experimental, (ii) theoretical and (iii) using computational fluid dynamics (CFD). Each of them has pros and cons, which can be evidenced during the usual design process of jet pumps. The theoretical approach is frequently used to estimate the diameter of its sections. However, this approach cannot give the lengths and shapes of these sections. Therefore, it is necessary to use experimental data to evaluate correlations that give optimized lengths and shapes (TOTEFF; TOVAR, 2018). Furthermore, the theoretical method is limited to a number of simplifying assumptions that fail in some designs conditions (like at high values of NXP distances), remaining the experimental and CFD techniques. CFD is a powerful tool to design jet pumps because it is possible to evaluate several geometries and apply optimization techniques to enhance ejector efficiency cheaply. Nevertheless, numerical models used in this process must be validated by experimental data. Since each part of the ejector needs to be manufactured, the experimental rig mounted and equipped with sensors, the conduction of such experiments is expensive. Currently, most of the literature uses CFD and theoretical analysis to jet pump studies, limiting the experiments to the validation step.

\subsection{Jet pump efficiency}

To define the jet pumps efficiency that works with incompressible fluids and same properties at secondary and primary inlets, it is often used the ratio of the work done by the ejector and the work provided to the ejector (eq. 1.5) (MUELLER, 1964):

$$
\eta=\frac{\dot{W}_{\text {Done }}}{\dot{W}_{\text {Input }}}
$$

Where $\eta$ is the efficiency and $\dot{W}$ is the work. The work done by the ejector is the multiplication of secondary inlet mass flow and the total pressure difference between the secondary inlet section and the outlet section. The work provided to the system is the multiplication of the 
primary inlet mass flow and the total pressure difference between the primary inlet section and the outlet section. Hence,

$$
\eta=\overbrace{\frac{\dot{m}_{\text {secondary }}}{\dot{m}_{\text {primary }}}}^{M} \cdot \overbrace{\frac{\left(P_{\text {outlet }}-P_{\text {secondary }}\right)}{\left(P_{\text {primary }}-P_{\text {outlet }}\right)}}^{N}
$$

In liquid jet pumps without a significant difference of temperature between the inlets, it is possible to vanish the implicit density present in the mass flow and simplify the ratio $M$. This term receives the symbol $M$. The ratio $N$ is called pressure efficiency and has the symbol $N$ assigned to it. Therefore,

$$
\eta=M \cdot N
$$

In the literature (STEPANOFF, 1957), it is often reported two kinds of curves to represent the behaviour of the ejector efficiency in different operating conditions. One of them is the plot of the total pressure efficiency $(N)$ as a function of the flow ratio $(M)$ and, the other one is the plot of the efficiency $(\eta)$ as a function of the flow ratio $(M)$. Figures 8 and 9 show the results obtained by Winoto, Li \& Shah (2000) of the curves quoted above for a jet pump tested with water at secondary and primary inlet, where is possible to observe good agreement between theoretical and experimental approaches. The experimental rig used by Winoto, Li \& Shah (2000) has a centrifugal pump to supply the high-pressure primary fluid, the secondary fluid was sucked from a large water tank, the pressures were measured by pressure gauges at the inlets and outlet, and gate valves control the fluxes at same locations. Figure 7 shown such experimental rig.

Figure 7 - Schematic representation of the experimental rig used by Winoto, Li \& Shah (2000).

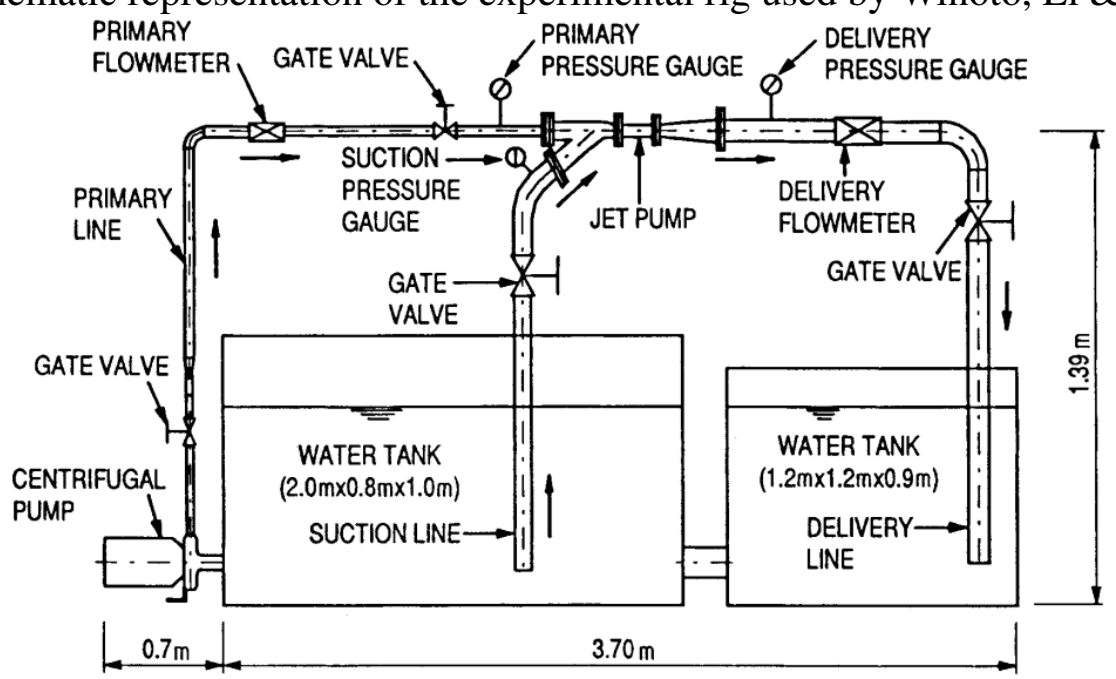

Source: (WINOTO; LI; SHAH, 2000). 
Figure 8 - Pressure efficiency as a function of the flow ratio.

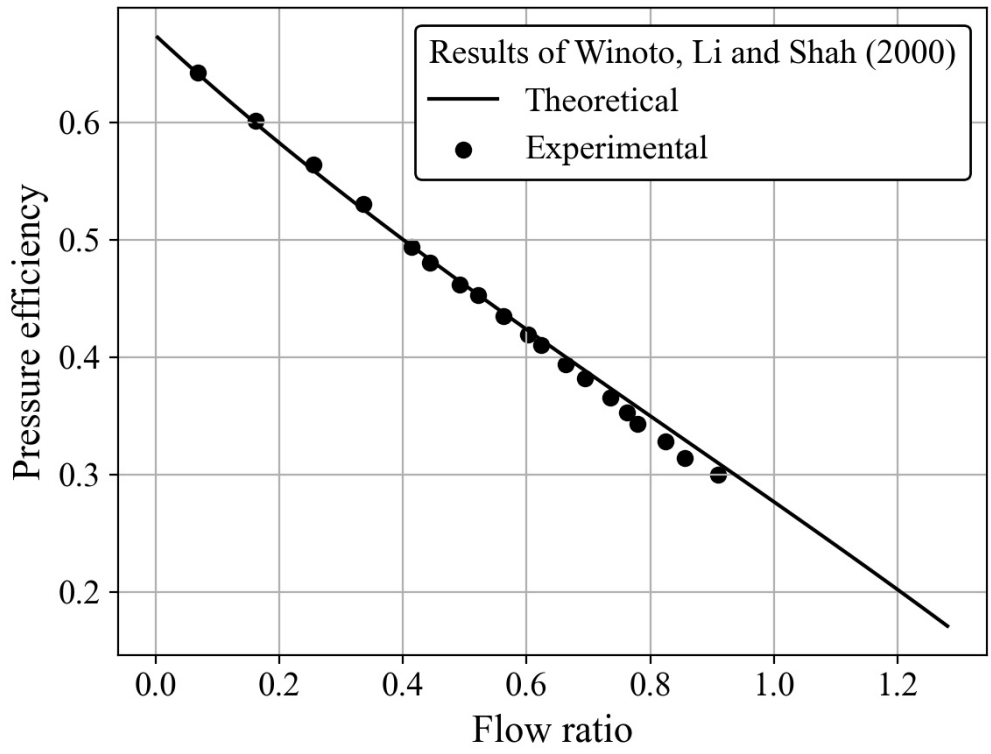

Source: Author.

Figure 9 - Pressure efficiency as a function of the flow ratio.

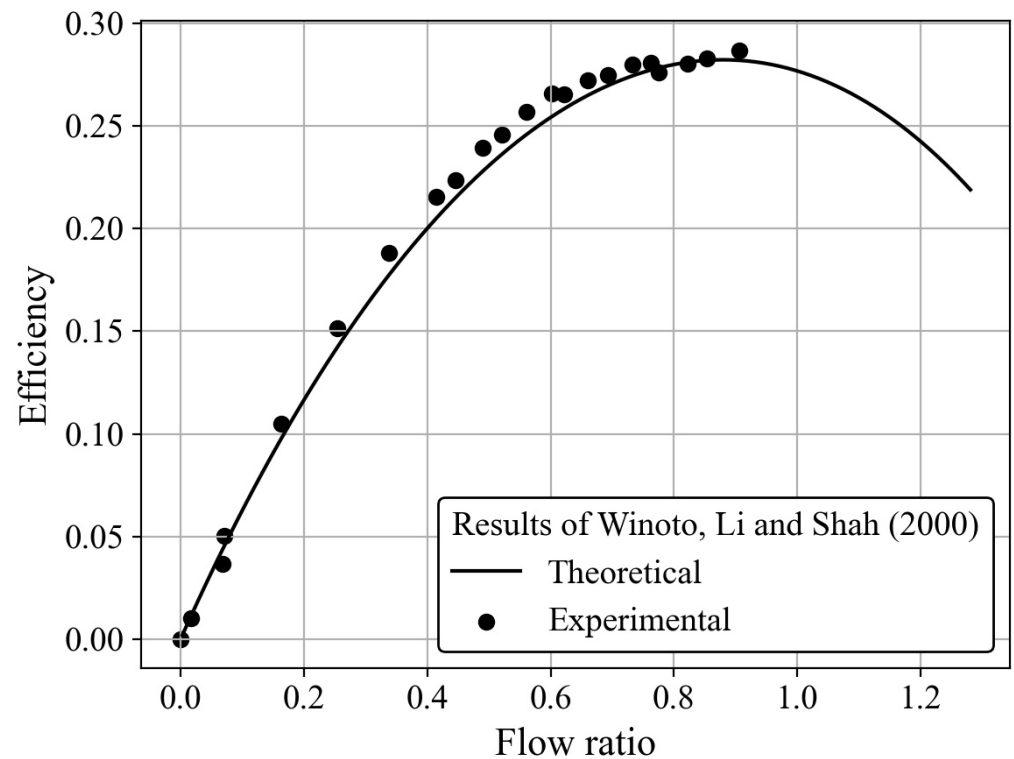

Source: Author.

In Figure 9, it is possible to see the typical parabolic behaviour of jet pumps. It can be explained since both the values of $N$ and $\eta$ are functions of the flow ratio. Additionally, losses at the main parts of the jet pump also exhibit a quadratic behaviour (SANGER, 1968). One can observe that the total pressure efficiency (Figure 8) curve can be approximated as a straight 
line. This approximation is valid for almost all jet pumps. However, depending on the operating conditions and the geometry, the curve can diverge from this behaviour. The decreasing aspect of the total pressure ratio is intuitive. As the flow ratio $(M)$ increases, the amount of energy given by the jet core is further dissipated (since the primary flow rate is kept constant and the secondary flow rate increases). Therefore the final specific energy is lower.

Moreover, it is essential to remember that all behaviour quoted so far is about incompressible and same fluids with the same properties at the primary and secondary inlet. The behavior of efficiency curves for Jet pumps that work with different fluids (but incompressible) at secondary and primary had the same behaviour (ABDOU; MIKHAIL; ABOU-ELLAIL, 2006).

For ejectors that work with biphasic flows, the efficiency curves also present the same behaviour. As the focus of the present dissertation is in the incompressible field, it is suggested to the reader the work of Cunningham \& Dopkin (1974) to further information about the biphasic ejectors.

\subsection{Topology optimization}

The optimization of engineering devices can be classified into three groups depending on these characteristics. Parametric optimization is the procedure that tries to maximize or minimize an objective function by changing the size of predetermined geometry without changing its form (1 in Figure 10). The shape optimization is made when the movement of the boundaries is allowed, the freedom to change the geometry is greater than the parametric. However, the final result still depends on an initial estimate ( 2 in Figure 10). The absence of an initial estimate characterizes the topology optimization that, to obtain a maximum or minimum of an objective function, only requires the boundary conditions, constraints, and the physics that rule the system. From these characteristics, the latter approach can produce non-intuitive geometries (3 in Figure 10). 
Figure 10 - The three major groups of optimization techniques.

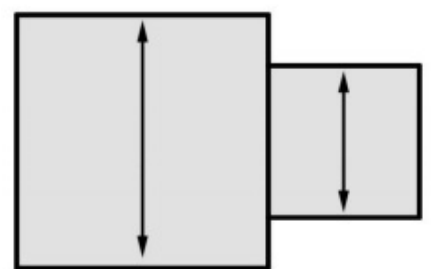

1) Parametric optimization

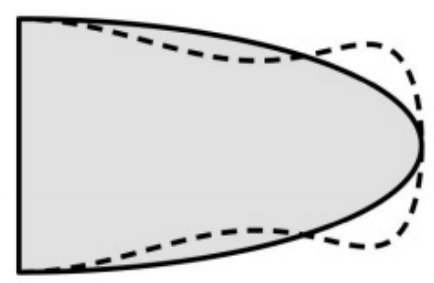

2) Shape optimization

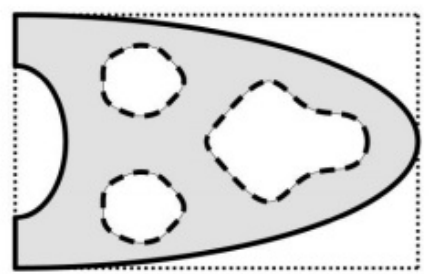

3) Topology optimization

Source: (ALLAIRE et al., 2019).

In the last decades, many techniques were developed to optimize several kinds of systems. Among them, gradient-based methods (GBM) and stochastic methods can be quoted as the most used (MITROPOULOU et al., 2013). In topology optimization, the related literature often uses the gradient methodology.

The stochastic, on the other hand, is frequently used in cases where the evaluation of derivatives is not possible, e.g. modeling of failures (ERMOLIEV; GAIVORONSKI, 1991). One example of this methodology is the class of genetic algorithm (HOLLAND et al., 1992), which mimics the nature with respect to genetic evolution and prioritizes the samples that generate the better results concerning objective functions to create other samples. However, the need to evaluate several system models makes this approach very costly depending on the complexity of the equations that rule the system.

The central core of all GBM methods is sensitivity analysis. It is a robust methodology to understand how the variation of the selected design parameters can influence an objective function that describes, somehow, the efficiency of the system (CACUCI et al., 1980). To this end, there are three significant approaches (PAPOUTSIS-KIACHAGIAS; GIANNAKOGLOU, 2016):

- Finite Difference (FD): The gradient is calculated simulating the whole system of governing equations with small differences in each design variables at a time. Despite the straightforward implementation, the computational cost grows with the number of design variables, so, in complex systems with a number of design variables, FD is prohibitive. Besides this, the results are dependent on how small the difference is (JAMESON, 1995; KIM; ALONSO; JAMESON, 1999).

- Direct Differentiation (DD): In this approach, the gradient is evaluated continuously or 
discretely. First, the total derivative of the objective function is derived, then the resulting system of equation is solved. This technique does not depend on the disturbance, but it still depends on the number of design variables (GIANNAKOGLOU; PAPADIMITRIOU, 2008).

- Adjoint method (AM): As the cost to evaluate the gradients using this approach does not depend on the number of variables, it is frequently used in the topology, and shape optimization (SUN; LIEBERSBACH; QIAN, 2020; YOON, 2020). Here, another system of equations, called adjoint equations, is solved, and the resulting variables are used to calculate the gradient. Depending on how this set of equations is derived, a different name is specified. If the equations are obtained from the continuous Navier-Stokes equations (NSE), the method is called the continuous adjoint method. On the other hand, if the equations are obtained from the discrete form of NSE, the method receives the name discrete adjoint method. (DILGEN et al., 2018a; GILES; PIERCE, 2000).

In the face of the number of design variables and the literature recommendation, the adjoint method was chosen to calculate the gradients in the present study, whose discrete type is

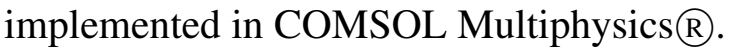

The topology optimization in fluid dynamics problems was first introduced by the pioneer work of Borrvall \& Petersson (2003). The authors proposed introducing a penalization parameter in the NSE to drive the flow variables for minimization or maximization of an objective function. The penalization parameter was defined in function of a design variable $\xi$. In that fashion, the node can be a solid domain when the $\xi$ value is equal to zero or a fluid domain when the $\xi$ value is equal to one. Nonetheless, in this methodology, the $\xi$ is relaxed by defining the variable between zero and one. This approach receives the name "density method".

The density method has some drawbacks like the intermediate values (also called grey elements) that have no physical meaning, spurious pressure inside the "solid" domain (since the penalization acts only on the velocity), and the loss of energy due to the flow in the intermediary values (CHALLIS; GUEST, 2009). Nevertheless, topology optimization using the density method has a powerful potential in the optimization field.

From the work of Borrvall \& Petersson (2003), which uses the Stokes flow (very low Reynolds), the technology advances to the full Navier-Stokes (i.e. with inertia forces) (GERSBORG-HANSEN; SIGMUND; HABER, 2005) and, currently, to problems involving 
turbulent flows (DILGEN et al., 2018a) and heat transfer (DILGEN et al., 2018b). Nonetheless, the closest application related to the scope of this study was made by Andreasen (2017), which used the 2D planar space and laminar flow assumptions to carry out the topology optimization of a device called by the author as "inertia-driven dosing units". Since the operational principle of this type of device and pressure field generated by the 2D planar assumption only resembles jet pumps, the latter has been barely exploited. This way, the present work has the objective to advance in the area of topology optimization applied to ejectors.

\subsection{Objective}

The Research Center for Gas Innovation (RCGI) supports the project "High-Efficiency Ejector for Gas Compression" (project number 38) where the present work is inserted. The project's goal is to enhance the efficiency of the biphasic ejectors up to their usage becomes more advantageous than the usual method applied to carbon dioxide compression. This way, despite the project goal to be a biphasic ejector, the present study will treat the jet pump flow as incompressible, laminar, adiabatic, without body forces and in steady state. Moreover, the same fluid and temperature was considered at primary and secondary inlet. This choice was based on the fact that this study proposes to advance in the new field of topology optimization applied to ejector technology, which has a number of gaps, i.e. lack of works involving optimization algorithms in the jet pump field. Thus, it was preferred to limit this study's scope, avoiding gathering too much complexity and its potential problems in only one work. Indeed, the application of this kind of device is limited to microfluidics application (e.g. mixing process). However, it can also be seen as a proof of concept that further studies (e.g., turbulent flow) can take advantage of. To summarize, the objectives of the present works are:

1. To conduct topology optimization of a laminar jet pump devices using as base the work of Andreasen (2017) and verify the limitations of the methodology used by this author (it is expected due to different goals between the device simulated in the base article and jet pumps). The simulations used up to this point will be called of "first group" or "first round".

2. Once found the methodology limitations by analysing the first round of simulations. Propose a enhancement of the optimization problem. This can be done introducing more restrictions or using a multi-objective function. 


\section{LITERATURE REVIEW}

\subsection{Search considerations}

For the literature survey, the term "jet pump" was chosen to search the references in the scientific repositories. Also, terms like "CFD” and "optimization" were eventually attached to the "jet pump" to filter the results to the main scope of this work (optimization of jet pumps using algorithms and CFD). However, it should be said that very few studies were found in the proposed field of the present work. Moreover, some references about the historical development cannot be reached either because it is in another language (e.g. Japanese and Russian) or because of the reference age.

\subsection{Literature review - jet pumps performance enhancing}

Some authors (LIU; ZHAN; LIA, 2017; PENG et al., 2017) mention a German scholar called G. Zehner as the first to study the physics involved in the ejector operation. In contrast, the first practical application of such a device was reported by Thomson (1852) to remove water from the pits of submerged water wells. Before the development of CFD codes, studies of jet pumps found in the literature were only related to experimental and theoretical analysis based on the work done by Rankine (1871). As the last approach cannot be used for optimization purposes, because the simplifying assumptions are linked to a previously determined design and cannot predict considerable changes in the geometry (SANGER, 1968), it is possible to consider the experimental research as the first attempts to optimize the jet pumps geometry. Figure 11 was built to help the interpretation of the subsequent discussion.

Figure 11 - Schematic representation of the dimension used in the literature review.

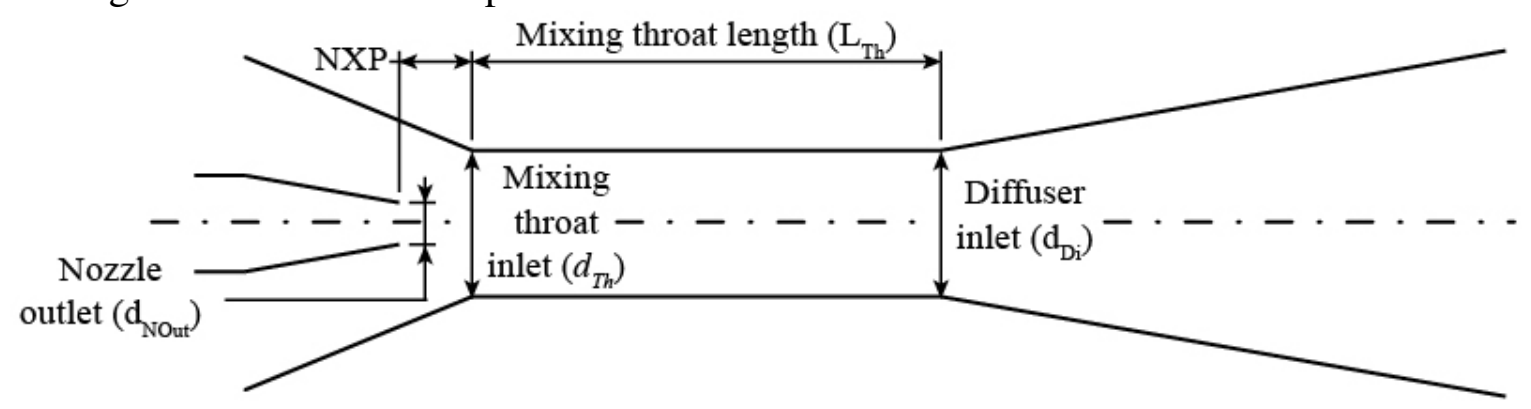

Source: Author. 
Cunningham (1957) performed experimental and theoretical analysis on jet pumps for oil lubrication systems in the aeronautical field. With the experimental data, it was possible to establish a correlation that gives the best $b$ (area ratio between the nozzle diameter and throat diameter) for each flow ratio $(M)$. The maximum efficiency of $29.8 \%$ was verified for values of $b$ equal to 0.3 and flow ratio equal of 0.8 . To the relation $S$, it was reported that each area ratio between the nozzle diameter and throat diameter has a range of optimum values that increase as the flow ratio $(M)$ increases and decrease when $b$ increases. Moreover, it was created an empirical correlation to determine $S$ as a function of $b$. The elliptical throat entrance profile produced greater values of efficiency. Nonetheless, the study does not entirely survey the influence of the throat lengths in the jet pump efficiency, from where other design relations and better efficiency could be obtained. Remembering that

$$
\begin{gathered}
b=\left(\frac{d_{\text {NOut }}}{d_{T h}}\right)^{2} \\
S=\frac{N X P}{d_{\text {NOut }}} \\
l=\frac{L_{T h}}{d_{T h}}
\end{gathered}
$$

Schulz \& Fasol (1958) found that the utilization of multiple nozzles (nozzle (D) in Figure 12) can reduce the optimum mixing length section up to 3.5 times the diameter of the same section. However, the maximum efficiency reduces, reaching maximum values around $30 \%$. When a single nozzle was utilized, the optimum efficiency reached was around $37 \%$ with mixing length around 7.5 mixing throat diameters.

Mueller (1964) conducted an experimental analysis to calibrate his analytical model that determines the efficiency of jet pumps. Further, it was investigated how the geometry dimensions change the ejector characteristics and efficiency. The results from his experiments pointed that (i) the $S$ dimension is not well defined, being the optimum value, indeed, defined in a range. However, the mean value of $S$ equal to one was recommended. (ii) with respect to the area ratio $(b)$, it was concluded that despite each area ratio has its efficiency as a function of flow ratio, the maximum values are reached when $b$ was between 0.44 and 0.55 . (iii) The optimum mixing chamber length was also defined in a range, and interdependence with the diffuser angle 
was established. The author summarized that, for an angle of five degrees (angle with better results), the optimum obtained relation was 7.5 times the diameter of the mixing section. (iv) bell-mouth section to the entrance and internal nozzle region presents the highest performance. The maximum efficiency record was $36.9 \%$.

Figure 12 - Schematic representation of the different types of nozzles tested in the literature.

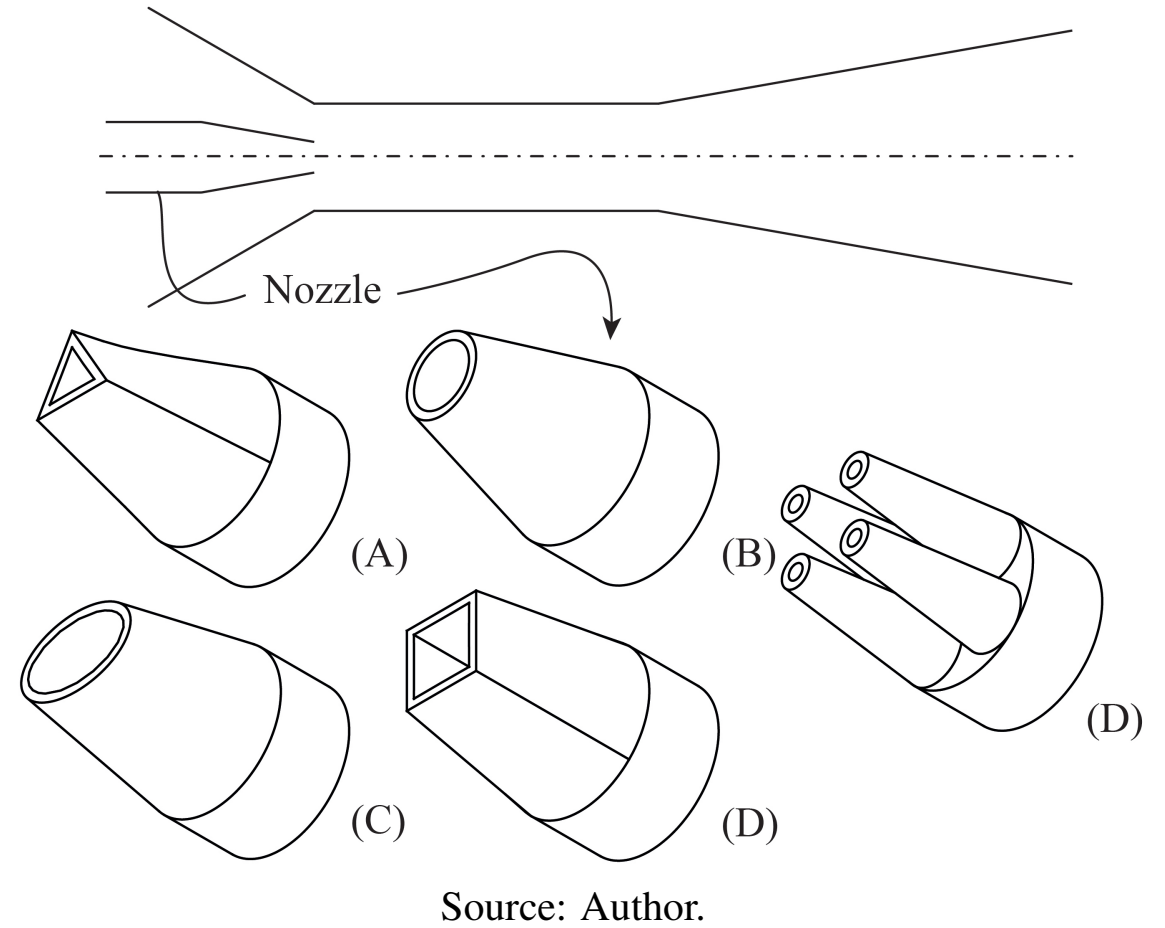

With an experimental rig, Reddy \& Kar (1968) tested several geometric configurations of separated components and assembled jet pumps. In face of the results, it was determined experimental coefficients used in their theoretical model and recommendations for optimum performance. As concluded by the author, this condition occurred when the driving nozzle with a geometry of a semicone had an angle between $8^{\circ}$ and $10^{\circ}$ (angle $\chi$ in Figure 13), the area ratio $(b)$ for jet pumps with fixed $S$ had values between 0.33 and 0.543 , the area ratio $(b)$ for jet pumps with variable $S$ had a value about 0.205 , the mixing throat length had 18 times the nozzle diameter, the angles of the diffuser had $5^{\circ}$ (angle $\varsigma$ in Figure 13) and the suction section had a cone angle between $20^{\circ}$ and $24^{\circ}$ (angle $\omega$ in Figure 13). The maximum efficiency reached in the article was $39.8 \%$. However, the authors stated that the value of $42 \%$ could be reached with proper material selection.

Cairns \& $\mathrm{Na}$ (1969) summarized extensive experimental investigation of optimum jet pump dimension and proposed a methodology to design optimum jet pumps dimensions for a given application. The maximum efficiency of $42 \%$ was obtained with the dimension of $b$ equal 
to 0.54 , throat length equal to 4.1 times the throat diameter, entrance angle equal to $40^{\circ}$ and diffuser angle equal to $6.5^{\circ}$. The author also reported that these values might vary in ranges that do not modify the efficiency significantly.

In face of jet literature results, Guillaume \& Judge (1999) tested jet pumps with elliptical nozzle cross-section (Figure 12 - (C)). Their results pointed that the change was significantly increased (reaching the maximum of six times the efficiency of conventional design). These results evidence that unusual geometries are a potential field to improve ejector efficiency.

Figure 13 - Schematic representation of jet pump angles.

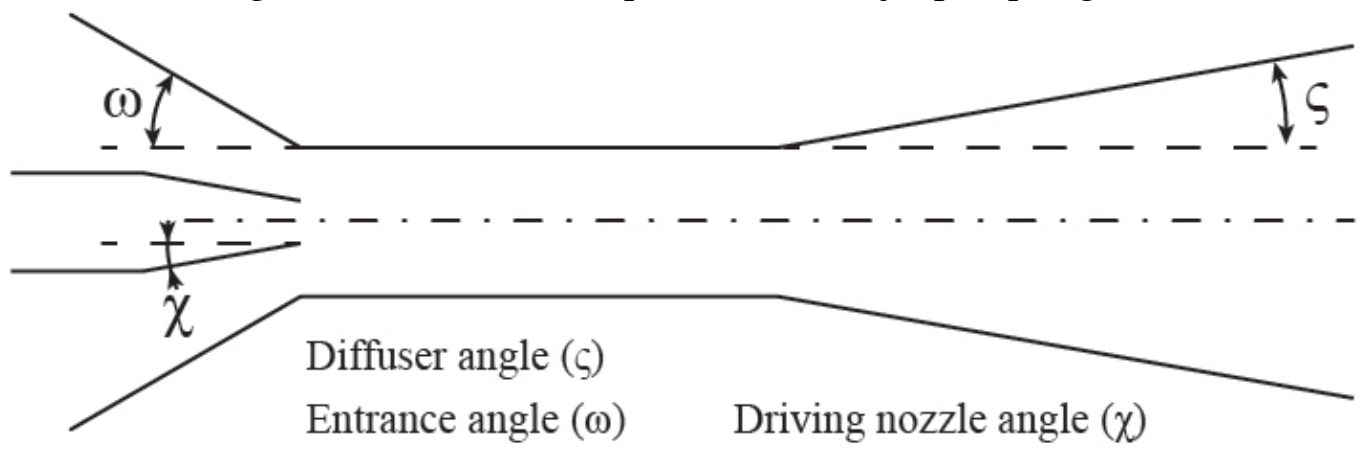

Source: Author.

Winoto, Li \& Shah (2000) experimentally investigated different sections of nozzle (e.g. triangular (A), circular (B), and squared (D) in Figure 12). The authors concluded that the circular one was the section that produces the lowest head losses, recommending this geometry for optimum ejector design.

Guillaume \& Judge (2004) introduced a swirling motion in the primary jet. The authors observed that it was possible to increase by up to $5 \%$ the ejector efficiency with this technique.

Narabayashi et al. (2006) investigated the effect of multiple nozzle ((D) nozzle in Figure 12) and tapered mixing throat $\left(d_{T h} \neq d_{D i}\right)$ section design with the conventional design of jet pumps. The simulations evidenced better performance when using the tapered mixing section and single nozzle. Moreover, the study showed that the ejectors are affected by the scale. The maximum efficiency with a $1 / 5$ model was about $27 \%$ to $32 \%$ for the $1 / 1$ model. Further, it was pointed that the jet pumps are considerably affected by the internal roughness, varying up to $4 \%$ of only due to its efficiency.

Long, Han \& Chen (2008) numerically investigated the interference of the thickness of the nozzle exit. The conclusion was that the variations of jet pump performance for the thickness were too slight (maximum of $1.3 \%$ ), and this variable can be neglected during the design 
process.

Yapici \& Aldas (2013) reported the lack of CFD studies in optimization involving jet pumps. To fill this gap in the literature, the authors conducted CFD simulation (validated by experimental data) using the finite volume method and turbulence models to investigate the mixing throat length of a jet pump with water at secondary and primary inlets, the $S$ distance and area ratio. The optimum relation found to the mixing length was the proportion of 7.4 times the throat diameter when $b$ equal to 0.13 . To the nozzle position, values of $S$ equal to 0.74 reached better values of efficiency for $b$ equal to 0.13 and optimum mixing throat length. Recommended value to $b$ equal to 0.22 was found. The maximum efficiency reached in the paper was $34.6 \%$. Additionally, the authors report the scale influence in the jet pump efficiency when they compared their results with other references.

As it is possible to observe, the results found in the literature do not converge to an optimum interval or specific value. Experiments show, at least, slightly different optimum values. Indeed, all the optimization studies quoted above have identical drawback. When the experiments or the simulation investigated any dimension, this dimension was the only change in the process, whereas the other ones were kept constant. This way, possible second-order interference behaviour and interdependence are not taken into account (SANGER, 1968). An optimization algorithm is a powerful approach to carry out a broad and more precisely search in the ejector parameters and overcome this drawback. However, the literature related to optimization algorithm applied to jet pump technology aided by CFD is scarce.

Dvorák (2006) conducted a shape optimization on the mixing, diffuser and inlet section of a jet pump. The simulations evidenced that the area ratio and diffuser section significantly impact the flow ratio (the objective function used in the study). The shape optimization smoothens the transition between the ejector parts, especially between the mixing and diffuser section. The simulation conducts the geometry to the tapered section. This result converges to the conclusion obtained by Narabayashi et al. (2006). The maximum efficiency of $30 \%$ was reached at $b$ equal to 0.3 ( $10 \%$ more than the conventional jet pump).

Liknes (2013) used the interior-point optimizer algorithm to optimize a jet pump used in the artificial lift technique. The design parameters chosen were primary pressure, primary density, primary flow rate $(M)$ and area ratio $(b)$. The results pointed that the optimized jet pump had better oil production than the base case without the jet pump and the case with a non-optimized 
jet pump. Further, the motive fluid rate consumption increases. The optimum area ratio found was 0.2. Nonetheless, the first three design parameters were operational ones, limiting the search for new geometry design-oriented algorithms.

Figure 14 - (A) Sketch of an inertia driven dosing unit. Left boundary is primary inflow. Right boundary is outlet. The secondary fluid enters from the lower port driven by the low static pressure inside the domain. (B) Result of the topology optimization conducted by Andreasen (2017).

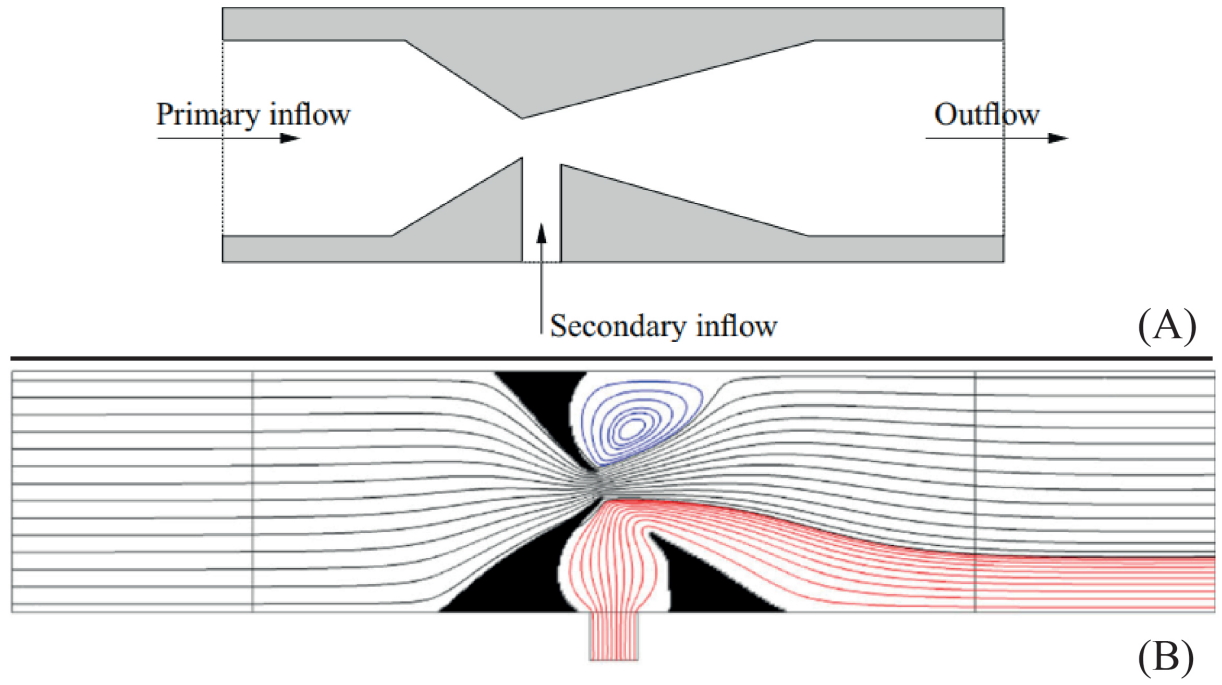

Source: Andreasen (2017).

Andreasen (2017) successfully developed a methodology for topology optimization on inertia driving dosing units (devices similar to the conventional jet pumps, however without throat and, as proposed by the author, with its secondary inlet positioned perpendicular to the primary inlet) in a laminar regime. In Figure 14, item (A) shows such a device. In his paper, the author presented how the geometry change with the variation of the constraint relaxation and pointed that, for some Reynolds number, the objective function do not produced satisfactory results. Although the author defined the domain in the 2D planar space, the paper had remarkable results ((B) in Figure 14), applicable optimization methodology and objective functions that can be used in the axisymmetric domain (closer to the jet pump technology). Because of it, the article was used as the basis for the development of the proposed study.

Toteff \& Tovar (2018) carried out the simulations of more than 400 jet pump models using the CFD software Ansys ${ }^{\circledR}$ aided by the optimization software Pipe-it ${ }^{\circledR}$ to enhance the performance of jet pumps applied in oil pipeline loops. The selected design variables were the nozzle diameter, throat length, throat diameter, NXP and pressure inlet. The results have shown the same behaviour pointed out by other authors for the design variables near the optimum values. 
In this region, several design variables have near the same performance with slight variation in their values. The optimized jet pump presents $17 \%$ better performance than the traditional design for the same parameter of consumed energy. In this case, the authors used the equation 2.4 as objective function to the optimization routine. Thus, as emphasized in Toteff \& Tovar (2018), they focus in uniform distribution of the flow in the two pipelines in parallel (1-3 line and 2-4 line as depicted in Figure 15) and not in the jet pump's efficiency.

$$
X=\left|\frac{\left|50-Q_{d e r}^{*}\right|}{50}-1\right| \cdot \frac{Q_{2}}{Q_{1}}
$$

With,

$$
Q_{d e r}^{*}=100 \cdot \frac{Q_{3}}{Q_{2}}
$$

Where $Q_{i}$ is the flow rate at the 1,2, 3 and 4 section of the system showed in Figure 15 and $X$ the objective function.

Figure 15 - Schematic representation of the .

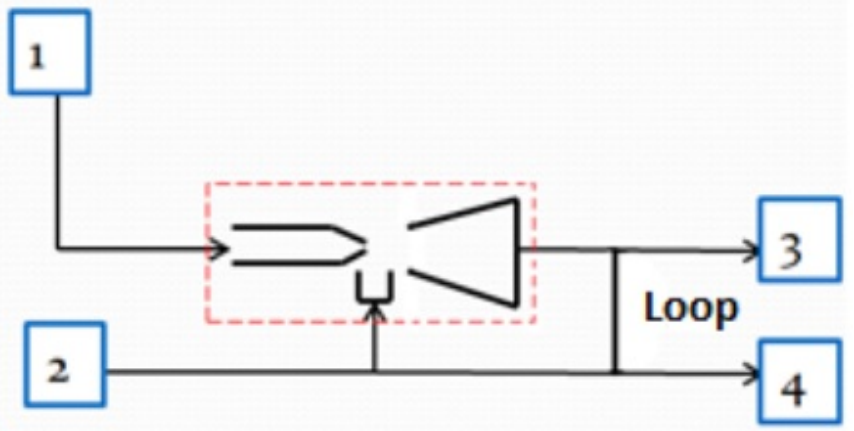

Source: Toteff \& Tovar (2018).

Hence, despite of the article treat about optimization of ejectors using a optimization algorithm and the design tends to have the same behaviour pointed by other authors, the efficiency neither was the main objective calculation non was presented. Therefore, the comparison with the other papers is not possible and optimized performance value given by Toteff \& Tovar (2018) are not show here. 


\section{METHODOLOGY}

\subsection{Governing equations}

\subsubsection{Continuity equation}

In the face of the assumption that the flow in this study was adiabatic, incompressible and fluid with same properties at both inlets, only the pressure and velocity fields were considered during the derivation of the governing equations. Following the methodology presented in Versteeg \& Malalasekera (2007), the analysis starts with a 2D infinitesimal element (Figure 16) with dimensions $2 d x$ and $2 d y$ and the flow variables stored at the centre of the element (where all the flow variables are functions of $x, y$ and $t$ ).

Figure 16 - Infinitesimal element used to the derivation with the flow variables stored at the element centre.

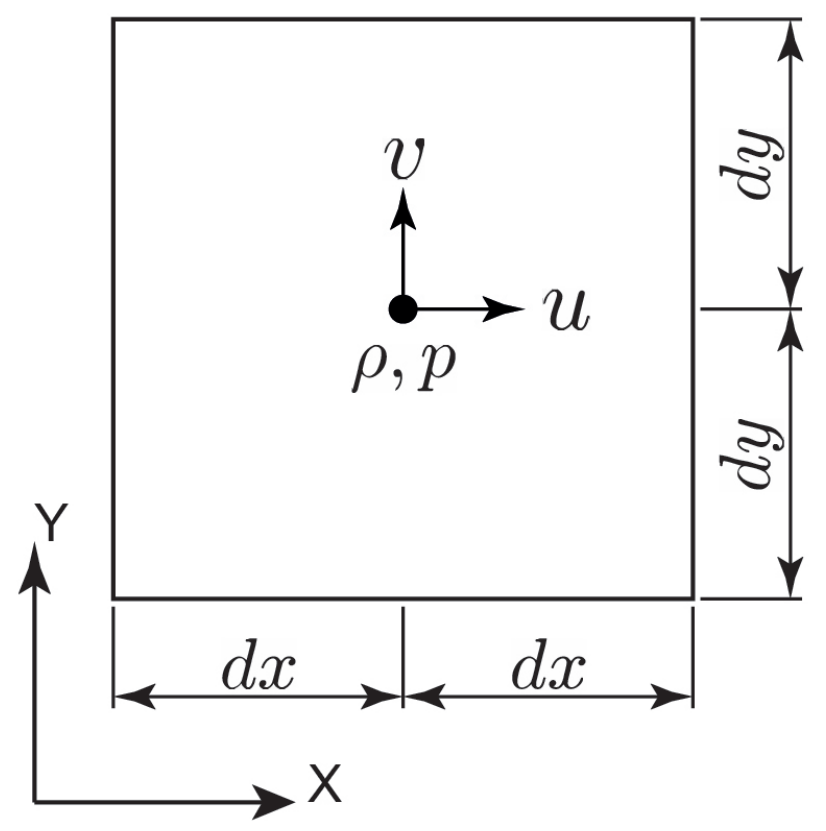

Source: Author.

On this element, the principles of mass and momentum conservation are applied. To this end, the flow variables stored in the centre of the element must be extrapolated to its boundaries. A way to do this is using the Taylor series, and, as the distances are infinitesimal, only the first order of the series can be used without error concern. 
Applying it to the principle of mass conservation

$\{$ Inlet mass flow ratio $\}-\{$ Outlet mass flow ratio $\}=\left\{\begin{array}{c}\text { Rate of mass increase } \\ \text { in the domain }\end{array}\right\}$

It is obtained the condition depicted in Figure 17.

Figure 17 - Mass flow at the boundaries of the domain.

$$
(v \rho) d x+\frac{1}{2} \frac{\partial(v \rho)}{\partial y} d x d y
$$

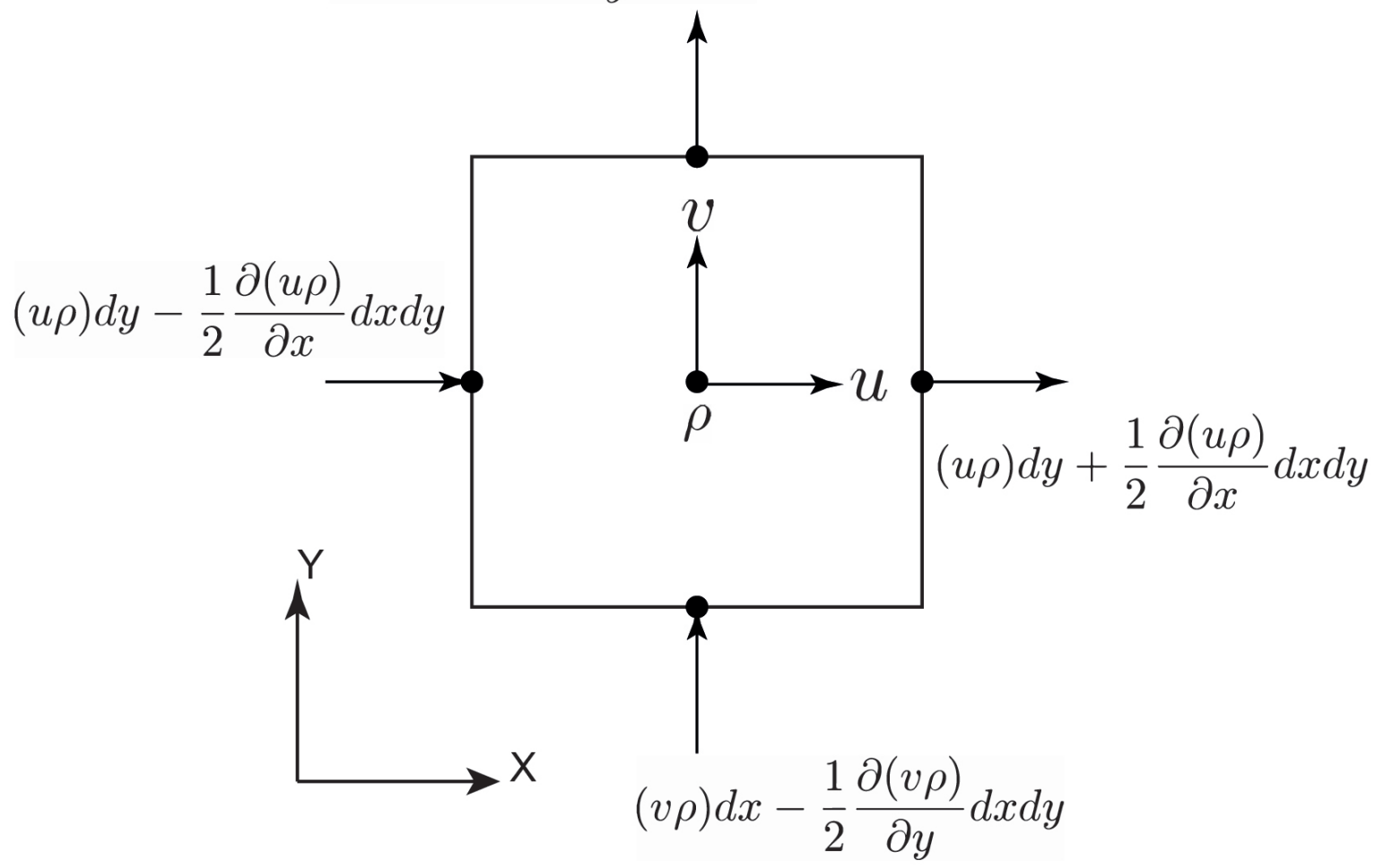

Source: Author.

Where $u$ is the velocity in the $x$-direction, $v$ is the velocity in the $y$ direction, and $\rho$ is the density. The minus sign is assigned to the flow that enters the domain and the plus sign to the outflow by convention. Therefore,

$$
\begin{array}{r}
(u \rho) d y+\frac{1}{2} \frac{\partial(u \rho)}{\partial x} d x d y-\left[(u \rho) d y-\frac{1}{2} \frac{\partial(u \rho)}{\partial x} d x d y\right]+(v \rho) d x+\frac{1}{2} \frac{\partial(v \rho)}{\partial y} d y d x- \\
{\left[(v \rho) d x-\frac{1}{2} \frac{\partial(v \rho)}{\partial y} d y d x\right]=\frac{\partial(\rho d x d y)}{\partial t}}
\end{array}
$$

Eliminating terms with opposite signs,

$$
\left.\left[\frac{1}{2} \frac{\partial(u \rho)}{\partial x}+\frac{1}{2} \frac{\partial(u \rho)}{\partial x}\right]+\frac{1}{2} \frac{\partial(v \rho)}{\partial y}+\frac{1}{2} \frac{\partial(v \rho)}{\partial y}\right] \cdot d x d y=\frac{\partial(\rho)}{\partial t} d x d y
$$


Then, rearranging the ones left, the continuity equation 3.4 is obtained. It is worth saying that the procedure presented above in $2 \mathrm{D}$ case can be extended to the $3 \mathrm{D}$ case.

$$
\frac{\partial(u \rho)}{\partial x}+\frac{\partial(v \rho)}{\partial y}=\frac{\partial(\rho)}{\partial t}
$$

Or, in symbolic representation

$$
\frac{\partial \rho}{\partial t}+\nabla \cdot(\rho \vec{u})=0
$$

With $\overrightarrow{\mathbf{u}}$ being the velocity vector. The density becomes constant with respect to the spatial coordinates and time using the incompressible, adiabatic and same fluid properties for both inlets flow field assumption. Thus, further possible simplifications result in the equation 3.6.

$$
\nabla \cdot \vec{u}=0
$$

Or, in the index notation:

$$
\partial_{i} u_{i}=0
$$

\subsubsection{Momentum equation}

Where $e_{i}$ is the basis vector elements. Also following the methodology presented in Versteeg \& Malalasekera (2007) and using the last property transported by the flow field proposed in this study, the momentum equation is derived. It is possible to determine the flow velocity field by applying the conservation principle of this quantity to the element. This way, using Newton's second law that states:

$\{$ Rate of momentum increase $\}=\{$ S ummation of the forces acting on the particle $\}$

In the same way, made for the continuity equation, the forces must be extrapolated from the centre of the element to its boundaries (such as depicted in Figure 18 to the force in $x$-direction). 
Figure 18 - Forces acting on the boundaries of the element.

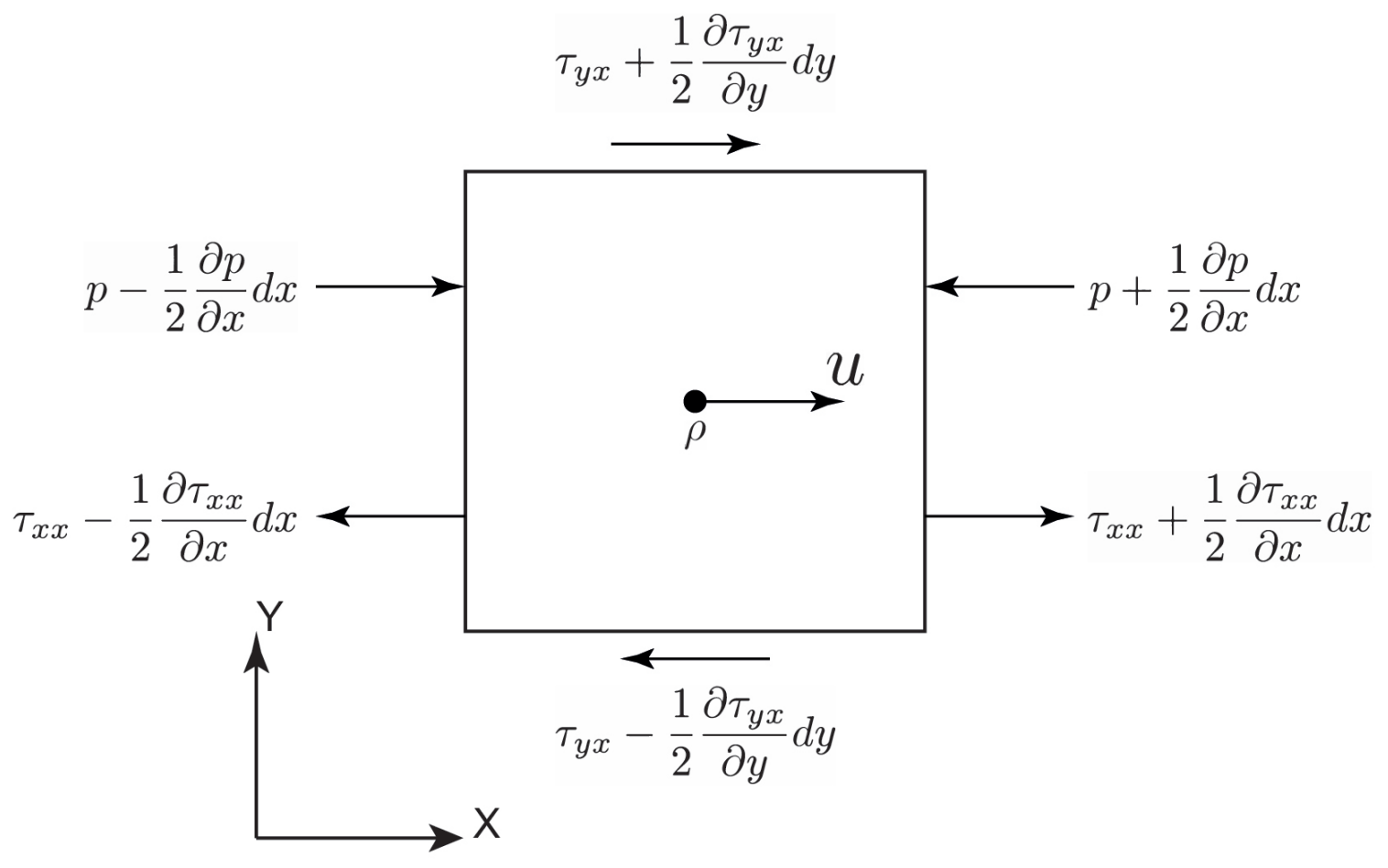

Source: Author.

Where $p$ is the static pressure, $\tau_{x x}$ the normal stress, $t$ the time and $\tau_{y x}$ the shear stress. From the Figure above, it is possible to see that no body forces were incorporated in the element. The reason for it comes from the simplifying assumptions where the absence of this kind of force was stated. Hence, taking place the summation of the forces:

$$
\begin{array}{r}
p d y-\frac{1}{2} \frac{\partial p}{\partial x} d x d y-\left[p d y+\frac{1}{2} \frac{\partial p}{\partial x} d x d y\right]-\left[\tau_{y x} d x-\frac{1}{2} \frac{\partial \tau_{y x}}{\partial y} d y d x\right]+\tau_{y x} d x+\frac{1}{2} \frac{\partial \tau_{y x}}{\partial y} d y d x+ \\
\tau_{x x} d y+\frac{1}{2} \frac{\partial \tau_{x x}}{\partial x} d x d y-\left[\tau_{x x} d y-\frac{1}{2} \frac{\partial \tau_{x x}}{\partial x} d x d y\right]=d x d y \rho \frac{D u_{1}}{D t}
\end{array}
$$

Eliminating terms with opposite signs

$$
\left[\frac{1}{2} \frac{\partial p}{\partial x}+\frac{1}{2} \frac{\partial p}{\partial x}+\frac{1}{2} \frac{\partial \tau_{y x}}{\partial y}+\frac{1}{2} \frac{\partial \tau_{y x}}{\partial y}+\frac{1}{2} \frac{\partial \tau_{x x}}{\partial x}+\frac{1}{2} \frac{\partial \tau_{x x}}{\partial x}\right] \cdot d x d y=d x d y \rho \frac{D u_{1}}{D t}
$$

And rearranging the ones left,

$$
\rho \frac{D u}{D t}=-\frac{\partial p}{\partial x}+\frac{\partial \tau_{y x}}{\partial y}+\frac{\partial \tau_{x x}}{\partial x}
$$

Similarly for the $y$ axis:

$$
\rho \frac{D v}{D t}=-\frac{\partial p}{\partial y}+\frac{\partial \tau_{x y}}{\partial x}+\frac{\partial \tau_{y y}}{\partial y}
$$


The shear stress terms were modelled as a function of velocity by Stokes in 1845 for Newtonian fluids (ANDERSON, 1995). Equations 3.13 to 3.15 present this model for incompressible fluids.

$$
\begin{gathered}
\tau_{x x}=2 \mu \frac{\partial u}{\partial x} \\
\tau_{y y}=2 \mu \frac{\partial v}{\partial y} \\
\tau_{x y}=\tau_{y x}=\mu\left[\frac{\partial v}{\partial x}+\frac{\partial u}{\partial y}\right]
\end{gathered}
$$

Where $\mu$ is the dynamic viscosity. Substituting the equations 3.13 to 3.15 in the equation 3.12 and using the continuity equation 3.6, it is obtained (in the vector form):

$$
\rho \frac{D \vec{u}}{D t}=-\nabla p+\mu \Delta \vec{u}
$$

It is worth saying that the procedure presented above in $2 \mathrm{D}$ case can be extended to the $3 \mathrm{D}$ case. The term $D \vec{u} / D t$ from the left side of the equation 3.6 is called Lagrangian derivative (or material derivative), in this case, of velocity, which means that the derivative is computed tracking the movement of the infinitesimal element as it moves with the stream. However, to describe all the possible paths, it would be needed a prohibitive number of equations. Alternatively, it is possible to use the Eulerian description of the continuum. This approach considers that the infinitesimal domain is fixed in the space, and the properties change as the flow pass through it. The name conservative form can be used to the equations that are fixed (Eulerian description) in the space and non-conservative to those that are moving with the stream (Lagrangian description) (ANDERSON, 1995).

The equivalence of these descriptions can be demonstrated by analysing an infinitesimal domain moving in the space transporting a generic quantity $\varphi$ as shown in Figure 19.

Expanding the point zero with the first order of the Taylor series and neglecting the high order terms:

$$
\varphi_{1}=\varphi_{0}+\left(\frac{\partial \varphi}{\partial x}\right)_{1}\left(x_{1}-x_{0}\right)+\left(\frac{\partial \varphi}{\partial y}\right)_{1}\left(y_{1}-y_{0}\right)+\left(\frac{\partial \varphi}{\partial t}\right)_{1}\left(t_{1}-t_{0}\right)
$$


Figure 19 - Infinitesimal domain moving with the stream where the property $\varphi$ is analyzed.

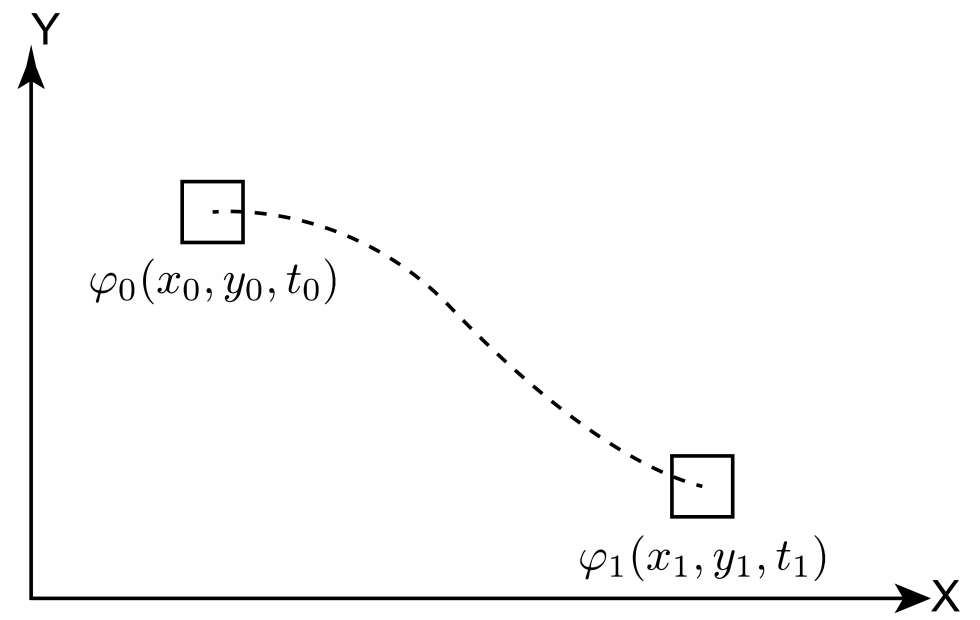

Source: Author.

Rearranging the terms, dividing the equation by $t_{2}-t_{1}$ and applying the limit:

$$
\lim _{t_{1} \rightarrow t_{0}} \frac{\varphi_{1}-\varphi_{0}}{t_{1}-t_{0}}=\left(\frac{\partial \varphi}{\partial x}\right)_{1} \lim _{t_{1} \rightarrow t_{0}} \frac{x_{1}-x_{0}}{t_{1}-t_{0}}+\left(\frac{\partial \varphi}{\partial y}\right)_{1} \lim _{t_{1} \rightarrow t_{0}} \frac{y_{1}-y_{0}}{t_{1}-t_{0}}+\left(\frac{\partial \varphi}{\partial t}\right)_{1}
$$

Hence,

$$
\frac{D \varphi}{D t}=\left(\frac{\partial \varphi}{\partial x}\right) u+\left(\frac{\partial \varphi}{\partial y}\right) v+\frac{\partial \varphi}{\partial t}
$$

Or,

$$
\frac{D \varphi}{D t}=\frac{\partial \varphi}{\partial t}+\vec{u} \cdot \nabla(\varphi)
$$

In equation 3.20, the first term of the right-hand side is the change of the variable $\varphi$ with respect to the time and, the second term, the variation of the variable due to its convection. Therefore, the velocity vector $\vec{u}$ is the velocity that transports the quantity $\varphi$. This conclusion is clear to the 3.20. Nevertheless, to the momentum equation, the transported property is another velocity vector, and the difference becomes less clear.

Substituting equation 3.20 into equation 3.16, after substituting the $\varphi$ variable by $\overrightarrow{\mathbf{u}}$ and assuming the steady state of the flow field, it is obtained the final form of the momentum equation (equation 3.21).

$$
\vec{u} \cdot \nabla \vec{u}=-\nabla p+\mu \Delta \vec{u}
$$

Or in the index notation,

$$
\rho u_{j} \partial_{j} u_{i} e_{i}=-\partial_{i} p e_{i}+\mu \partial_{j} \partial_{j} u_{i} e_{i}
$$


Where $e_{i}$ is the basis vector elements. Equation 3.22 is called the Navier-Stokes equation. However, in the CFD community, the set of equations composed by equations 3.7 and 3.22 is often called Navier-Stokes equations (NSE) as well.

\subsection{Numerical approach}

In absence of an analytic and generic solution of the Navier-Stokes equations (NSE), the use of numerical methods is required (ÇENGEL; CIMBALA, 2013). The most used numerical approaches reported in the literature are the finite difference method (FDM), the finite element method (FEM) and the finite volume method (FVM). Each of them has its pros and cons, but there is no consensus in the CFD community on which is better to solve the NSE (MALISKA, 2017).

In this work, the well established in topology optimization problems (PICELLI; SIVAPURAM; XIE, 2021; ANDREASEN, 2017; ANDREASEN; GERSBORG; SIGMUND, 2009), commercial software COMSOL Multiphysics ${ }^{\circledR}$, which uses the finite element approach, was chosen to solve the Navier-Stokes equations and the subsequent optimization problem. The reason for using this software lies in the fact that the topology optimization, solution of the governing equations and optimization algorithms are all already implemented. This way, it was possible to accomplish, within the available time, what was initially proposed in this study.

\subsubsection{Finite element method}

In the FEM analysis, the initial domain is divided in $K$ elements with known dimension and spatial position with respect to a predetermined coordinate frame. In each element, it is sought for the approximate solution $h^{\delta}$ of the dependent variable $h$ of a partial deferential equation that rules the studied physics defined in $\Omega$ domain with $\Gamma$ boundary conditions (REDDY, 2004). Such approximation can be written as equation 3.23.

$$
h(x, y) \approx h^{\delta}(x, y)=\sum_{j=1}^{K} c_{j} \phi_{j}(x, y)+\phi_{0}(x, y)
$$

Where the term $\phi_{j}(x, y)$ is called the trial function, shape function or basis function, $\phi_{0}(x, y)$ is any function defined in such way that its values at the boundaries $(\Gamma)$ match with its exact value $\psi$ and $c_{j}$ is the approximated value of the dependent variable at the nodes. However, 
the trial function needs to be equal to zero at boundaries. Therefore, it is possible to write $\left.\phi_{j}(x, y)\right|_{\Gamma}=0$ and $\left.\phi_{0}(x, y)\right|_{\Gamma}=\psi($ KARNIADAKIS; SHERWIN, 2013).

Given any partial differential equation $\mathfrak{J}(h)=0$ and substituting $h$ by the approximation defined by equation 3.23, it is obtained the residual form of the $\mathfrak{J}(h)$ (equation 3.24).

$$
R\left(h^{\delta}\right) \neq 0
$$

Or,

$$
R\left(x, y, c_{j}\right) \neq 0
$$

As expected, the approximation generates solutions that are not zero. Several techniques are used to find the values of $c_{j}$ such that the results remain closer to the desired value, reducing as much as possible this difference. For each of these techniques is given a name. For example, if $R$ is made equal to zero at $\mathrm{N}$ points in the domain, the name collocation is attached to this approach (ZIENKIEWICZ; MORGAN; MORGAN, 2006).

The finite element method tries to force the zero value by applying the weighted residuals in $R$. Therefore,

$$
\int_{\Omega} w_{i}(x, y) R\left(h^{\delta}\right) d \Omega=0
$$

Where $w_{i}$ is an arbitrary, linear and independent function called the weight function or interpolation function. The equation 3.26 can also be written as the inner product of $w_{i}$ and $R\left(x, y, c_{j}\right)$ (equation 3.27).

$$
\left(w_{i}(x, y), R\left(h^{\delta}\right)\right)=0
$$

It is interesting to observe that other numerical methods can be obtained by selecting the appropriate weighted function (REDDY; GARTLING, 2010). Table 1 summarizes the option for the weight function and the names of its corresponding methods.

The COMSOL Multiphysics ${ }^{\circledR}$ uses the Continuous Galerkin method to solve the NSE (COMSOL, 2019). Thus, only this approach will be further described for the solution of the governing equations. 
Table 1 - Numerical methods and its respective weights.

\begin{tabular}{cc}
\hline Numerical method & Weighted function \\
\hline $\begin{array}{c}\text { Petrov-Galerkin } \\
\text { Galerkin }\end{array}$ & $w_{i}(x)=\psi(x) \neq \phi_{j}(x)$ \\
Least squares & $w_{i}(x)=\phi_{j}(x)$ \\
Collocation & $w_{i}(x)=\frac{\mathrm{d}}{\mathrm{d} x}\left(a(x) \frac{\mathrm{d} \phi_{i}}{\mathrm{~d} x}\right)$ \\
Finite Volumes & $w_{i}(x)=\delta\left(x-x_{i}\right)$ \\
& $w_{i}(x)=\left\{\begin{array}{l}1, \text { inside the element } \Omega^{j} \\
0, \text { outside of the element }\end{array}\right.$ \\
\hline
\end{tabular}

Source: Reddy \& Gartling (2010).

\subsubsection{Numerical solution of the Navier-Stokes equation}

Using the equations 3.7 and 3.22 and the methodology of finite element analysis, the governing equations were transformed from a set of partial differential equations into a system of algebraic equations, thus, enabling its solution. The steps to this end are described as presented by Reddy \& Gartling (2010).

Initially, it is carried out the inner product of the momentum and continuity equations with a weight function (equations 3.28 and 3.29) over a typical element $\left(\Omega^{e}\right)$ of the discretized domain.

$$
\begin{gathered}
\int_{\Omega^{e}} w_{i} \cdot\left[\rho u_{j} \partial_{j} u_{i}-\partial_{j} T_{i j}\right] d \Omega^{e}=0 \\
\int_{\Omega^{e}} Q \partial_{i} u_{i} d \Omega^{e}=0
\end{gathered}
$$

Where $Q$ is the weight function of the pressure dependent variable and $T_{i j}$ is the stress tensor (used to simplify the notation), defined for an incompressible Newtonian fluid as:

$$
T_{i j}=-p \delta_{i j}+2 \mu D_{i j}
$$

And,

$$
D_{i j}=\frac{1}{2}\left(\frac{\partial u_{i}}{\partial x_{j}}+\frac{\partial u_{j}}{\partial x_{i}}\right)
$$

Using the dimensional analysis, Reddy (2004) argues that there is a physical meaning for 
the weight functions as follows:

$$
w_{i} \sim u_{i} \quad Q \sim p
$$

The importance of such interpretation becomes apparent when the approximations of the dependent variables are imposed. This way, for the $w_{i}$, the approximations of velocity are used, whereas, for $Q$, the pressure approximation is employed. Taking the integration by parts of the momentum equation, results in the equation 3.33.

$$
\int_{\Omega^{e}}\left[w_{i} \rho u_{j} \partial_{j} u_{i}+\partial_{j} w_{i} T_{i j}\right] d \Omega^{e}=\oint_{\Gamma^{e}} T_{i j} w_{i} n_{j} d \Gamma^{e}
$$

Or yet,

$$
\begin{aligned}
\int_{\Omega^{e}}\left[w_{i} \rho u_{j} \partial_{j} u_{i}-p \partial_{i} w_{i}+\mu \partial_{j} w_{i}\left(\frac{\partial u_{i}}{\partial x_{j}}+\frac{\partial u_{j}}{\partial x_{i}}\right)\right] d \Omega^{e} & \\
& \oint_{\Gamma^{e}}\left[-\delta_{i j} p+\mu\left(\frac{\partial u_{i}}{\partial x_{j}}+\frac{\partial u_{j}}{\partial x_{i}}\right)\right] n_{j} w_{i} d \Gamma^{e}
\end{aligned}
$$

The right-hand side of equation 3.34 is called natural boundary condition, because it appears in the equation during its deduction and does not need to be imposed. In the case of NSE, the natural boundary corresponds to a prescribed tension.

In the continuity equation, it is inserted the minus signal to force the symmetry of the finite element model (ZIENKIEWICZ; MORGAN; MORGAN, 2006).

$$
-\int_{\Omega^{e}} Q \partial_{i} u_{i} d \Omega^{e}=0
$$

As it is possible to see in the equations 3.33 , the procedure is only conducted when it is possible to take advantage of the results, that is, the boundary term that gives rise after the integration by parts has a physical meaning and the derivative order of the dependent variable reduces (REDDY; GARTLING, 2010). The equations 3.34 and 3.35 are called the weak form of the Navier-Stokes equation.

Since the Galerkin method was chosen, the weight function is constrained to the same function selected for the dependent variables.

$$
u_{i}(\mathbf{X}) \approx \sum_{n=1}^{P_{p}} \hat{u}_{i}^{n} \psi_{n}(\mathbf{X})=\boldsymbol{\Psi}^{T} \hat{\mathbf{u}}_{i}
$$




$$
p(\mathbf{X}) \approx \sum_{n=1}^{Q_{p}} \hat{p}_{n} \phi_{n}(\mathbf{X})=\mathbf{\Phi}^{T} \hat{\mathbf{p}}
$$

Where $P_{p}$ is the polynomial order of the test and weight functions of velocity approximation, $Q_{p}$ is the polynomial order of the test and weight functions of pressure approximation, $\hat{u}_{i}$ is the nodal values of velocity in $i$ direction and $\hat{p}$ is the nodal values of pressure. The last terms of equations 3.36 and 3.37 are the vector notation used to simplify the handling of equations 3.34 and 3.35. Replacing the approximations written in the equations 3.36 and 3.37 in the dependent variable and weight functions of NSE's weak form, it is obtained:

$$
-\left[\int_{\Omega^{e}} \boldsymbol{\Phi} \partial_{i} \Psi^{T} d \mathbf{X}\right] \hat{\mathbf{u}}_{i}=0
$$

Where it is important to note that the pressure in the continuity is present inside the $\boldsymbol{\Phi}$ term.

$$
\begin{array}{r}
{\left[\int_{\Omega^{e}} \rho \boldsymbol{\Psi}\left(\boldsymbol{\Psi}^{T} \hat{\mathbf{u}}_{j}\right) \partial_{j} \boldsymbol{\Psi}^{T} d \mathbf{X}\right] \hat{\mathbf{u}}_{i}+\left[\int_{\Omega^{e}} \mu \partial_{j} \boldsymbol{\Psi} \partial_{j} \boldsymbol{\Psi}^{T} d \mathbf{X}\right] \hat{\mathbf{u}}_{i}+\left[\int_{\Omega^{e}} \mu \partial_{j} \boldsymbol{\Psi} \partial_{i} \boldsymbol{\Psi}^{T} d \mathbf{X}\right] \hat{\mathbf{u}}_{j}-} \\
{\left[\int_{\Omega^{e}} \partial_{i} \boldsymbol{\Psi} \boldsymbol{\Phi}^{T} d \mathbf{X}\right] \mathbf{p}=\left\{\oint_{\Gamma^{e}} \tau_{i} \boldsymbol{\Psi} d s\right\}}
\end{array}
$$

Where the term $\tau_{i}$ summarize the input parameter that represents a prescribed tension (left side of equation 3.34), moreover, it is possible to gather equations 3.38 and 3.39 in the matrix form, which is used to perform the calculations.

$$
\left[\begin{array}{ccc}
\mathbf{C}(\hat{\mathbf{u}}) & \mathbf{0} & \mathbf{0} \\
\mathbf{0} & \mathbf{C}(\hat{\mathbf{u}}) & \mathbf{0} \\
\mathbf{0} & \mathbf{0} & \mathbf{0}
\end{array}\right]\left\{\begin{array}{l}
\hat{\mathbf{u}}_{\mathbf{1}} \\
\hat{\mathbf{u}}_{\mathbf{2}} \\
\mathbf{p}
\end{array}\right\}+\left[\begin{array}{ccc}
2 \mathbf{K}_{11}+\mathbf{K}_{22} & \mathbf{K}_{12} & -\mathbf{Q}_{\mathbf{1}} \\
\mathbf{K}_{21} & \mathbf{K}_{11}+2 \mathbf{K}_{22} & -\mathbf{Q}_{2} \\
-\mathbf{Q}_{1}^{T} & -\mathbf{Q}_{2}^{T} & \mathbf{0}
\end{array}\right]\left\{\begin{array}{l}
\hat{\mathbf{u}}_{\mathbf{1}} \\
\hat{\mathbf{u}}_{\mathbf{2}} \\
\mathbf{p}
\end{array}\right\}=\left\{\begin{array}{l}
\mathbf{F}_{1} \\
\mathbf{F}_{2} \\
\mathbf{0}
\end{array}\right\}
$$

With,

$$
\begin{array}{cc}
C(u)=\int_{\Omega^{e}} \rho \boldsymbol{\Psi}\left(\boldsymbol{\Psi}^{T} \hat{\mathbf{u}}_{j}\right) \partial_{j} \boldsymbol{\Psi}^{T} d \mathbf{X} & K_{i j}=\int_{\Omega^{e}} \mu \partial_{i} \boldsymbol{\Psi} \partial_{j} \boldsymbol{\Psi}^{T} d \mathbf{X} \\
Q_{i}=\int_{\Omega^{e}} \partial_{i} \boldsymbol{\Psi} \boldsymbol{\Phi}^{T} d \mathbf{X} & F_{i}=\oint_{\Gamma^{e}} \tau_{i} \boldsymbol{\Psi} d s
\end{array}
$$

The selection of the interpolation functions needs special attention. Because the weight function applied in the weak form of continuity equation has no integral boundary terms, it is inferred that the pressure is a secondary variable. Hence, the variable $p$ must not be continuous. This behaviour is related to the Ladyzhenskaya-Babuška-Brezzi condition (LADYZHENSKAYA, 1969) which states the necessity of the interpolation function of the pressure variable be one order less than used for the velocity variable. Nonetheless, the COMSOL 
Multiphysics ${ }^{\circledR}$ uses the Galerkin/least squares (GLS) (HAUKE; HUGHES, 1994) stabilization on the weak form of the equations, what makes possible to use a linear interpolation function to the velocity and pressure variables. In FEM, it is usual to compactly represents the order (n) of the polynomials (P) by "Pn". For the case of NSE, the information of velocity polynomial goes first followed by the pressure polynomial information, so, for $\mathrm{P} 1+\mathrm{P} 1$, it means that a linear polynomial was used for both velocity and pressure approximations.

\subsection{Topology optimization - The density approach}

Before treating the solution of the equation, the flow field must be parametrized to make possible the optimization process. The method adopted to this end was first introduced by Borrvall \& Petersson (2003) where it is used a source term called permeability ( $\alpha$ ) (which can be interpreted as the opposite of Darcy porosity), written as a function of the design variables $\xi$, that acts on the velocity fluid. This way, when the design parameter is equal to one, the permeability assume its maximum value $\left(\alpha_{\max }\right)$ meaning that this region is a fluid domain and, when the design parameter is equal to zero, the permeability assume its minimum value $\left(\alpha_{\min }\right)$ meaning that the region is a solid domain. From equation 3.22 and inserting the design parameter, it is obtained:

$$
\rho u_{j} \partial_{j} u_{i} e_{i}=-\partial_{i} p e_{i}+\mu \partial_{j} \partial_{j} u_{i} e_{i}-\alpha(\xi) u_{i}
$$

In theory, the permeability ( $\alpha$ ) must have a infinity value when $\xi$ is equal to one. However, due to numerical issues, a finite value is used. The interpolation between the maximum and minimum values of $\alpha$ is given by equation 3.43 (BORRVALL; PETERSSON, 2003).

$$
\alpha(\xi)=\alpha_{\max }+\left(\alpha_{\min }-\alpha_{\max }\right) \xi \frac{1+q}{\xi+q}
$$

The presence of intermediary values of the design variable $\xi$ has no physical meaning. So, the penalization parameter $q$ is used in equation 3.43. For large value of $q$ (e.g. 100), the intermediaries values of $\xi$ are very penalized and the resultant geometry tends to converge to discrete values of $\xi$ (i.e. 0 and 1). Conversely, if a small value of $q$ is used (e.g. 0.001), the intermediaries values of $\xi$ are not so penalized and the optimized geometry tends do have these elements. The minimum value of alpha $\alpha_{\min }$ was set up equal to zero and its maximum value $\alpha_{\text {max }}$ equal to $10^{6} \mathrm{~Pa} \cdot \mathrm{s} / \mathrm{m}^{2}$. The hypothetical fluid used in the simulations has dynamic viscosity equal to $1.5 \mathrm{~Pa} \cdot \mathrm{s}$ and density equal to $30 \mathrm{~kg} / \mathrm{m}^{3}$. 
And the linear term of the matrix system 3.40 becomes:

$$
\left[\begin{array}{ccc}
\mathbf{C}(\hat{\mathbf{u}}) & \mathbf{0} & \mathbf{0} \\
\mathbf{0} & \mathbf{C}(\hat{\mathbf{u}}) & \mathbf{0} \\
\mathbf{0} & \mathbf{0} & \mathbf{0}
\end{array}\right]\left\{\begin{array}{l}
\hat{\mathbf{u}}_{\mathbf{1}} \\
\hat{\mathbf{u}}_{\mathbf{2}} \\
\mathbf{p}
\end{array}\right\}+\left[\begin{array}{ccc}
2 \mathbf{K}_{11}+\mathbf{K}_{22}+\mathbf{A}_{11} & \mathbf{K}_{12} & -\mathbf{Q}_{1} \\
\mathbf{K}_{21} & \mathbf{K}_{11}+2 \mathbf{K}_{22}+\mathbf{A}_{22} & -\mathbf{Q}_{2} \\
-\mathbf{Q}_{1}^{T} & -\mathbf{Q}_{2}^{T} & \mathbf{0}
\end{array}\right]\left\{\begin{array}{l}
\hat{\mathbf{u}}_{1} \\
\hat{\mathbf{u}}_{2} \\
\mathbf{p}
\end{array}\right\}=\left\{\begin{array}{l}
\mathbf{F}_{1} \\
\mathbf{F}_{2} \\
\mathbf{0}
\end{array}\right\}
$$

With,

$$
A_{i j}=\int_{\Omega^{e}} \boldsymbol{\Psi}^{T} \boldsymbol{\Psi} \alpha(\xi) d \mathbf{X}
$$

The results from the straight use of the design variable $\xi$ are susceptible to be mesh dependent so that the mesh refinement can give rise to new geometries instead of enhancing the numerical results of a coarser mesh. To handle it, the Helmholtz filter proposed by (LAZAROV; SIGMUND, 2011) was used (defined by the equation 3.47), which introduces a minimum length scale in the design domain.

$$
-r^{2} \nabla^{2} \hat{\xi}+\hat{\xi}=\xi
$$

The filter can be interpreted as a diffusion of the design variable so that it becomes defined in a region and no more in the discrete points. The intensity of diffusion is controlled by the term $r$. However, as a consequence of applying the filter, the region with intermediary design variables is further extended. This problem can be mitigated by applying the hyperbolic projection (WANG; LAZAROV; SIGMUND, 2011)

$$
\breve{\xi}=\frac{\tanh (\beta \lambda)+\tanh (\beta(\hat{\xi}-\lambda))}{\tanh (\beta \lambda)+\tanh (\beta(1-\lambda))}
$$

Then,

$$
\alpha(\breve{\xi})=\alpha_{\max }+\left(\alpha_{\min }-\alpha_{\max }\right) \breve{\xi} \frac{1+q}{\breve{\xi}+q}
$$

Where the term $\beta$ controls how sharp is the transition between the solid and fluid regions, the parameter $\lambda$ is the threshold of the projection, that, in all simulations, it was set up the value of 0.5 . Therefore, it is possible to rewrite the linear term 3.45 as:

$$
A_{i j}=\int_{\Omega^{e}} \boldsymbol{\Psi}^{T} \boldsymbol{\Psi} \alpha(\breve{\xi}) d \mathbf{X}
$$




\subsection{Solver - Fluid flow problem}

To solve the discretized Navier-Stokes equations in the steady-state and laminar flow, the commercial software COMSOL Multiphysics ${ }^{\circledR}$ uses the fully coupled Newton method with damping factor (COMSOL, 2019). The method is described as follows (REDDY; GARTLING, 2010). Given a flow problem, the discretized set of NSE (matrices in 3.45) can be written in compact form (equation 3.50).

$$
\widetilde{\mathbf{K}}(\mathbf{U}) \mathbf{U}=\widetilde{\mathbf{F}}(\mathbf{U})
$$

Where $\mathbf{U}$ is the vector that contains all the flow variables, $\widetilde{K}$ contains the terms of diffusion, advection, optimization variable and the incompressibility constraint. The term $\widetilde{F}$, at the righthand side of equation 3.50, has the boundary conditions. Further, defining the residual equation $R(U)$ by the equation 3.51 .

$$
\mathbf{R}(\mathbf{U})=\widetilde{\mathbf{K}}(\mathbf{U}) \mathbf{U}-\widetilde{\mathbf{F}}(\mathbf{U})=0
$$

Next, the Taylor expansion is applied in $R(U)$ about the known solution $U^{n}$. Assuming that the known solution has residue different from zero and the solution with $U^{n+1}$ has no residue, it is possible to derive the relation 3.52 .

$$
\begin{aligned}
& \mathbf{R}\left(\mathbf{U}^{n+1}\right)=\mathbf{R}\left(\mathbf{U}^{n}\right)+\left(\frac{\partial \mathbf{R}}{\partial \mathbf{U}}\right)_{\mathbf{U}^{n}} \Delta \mathbf{U}+O(\Delta \mathbf{U})^{2} \\
& 0=\mathbf{R}\left(\mathbf{U}^{n}\right)+\left(\frac{\partial \mathbf{R}}{\partial \mathbf{U}}\right)_{\mathbf{U}^{n}} \Delta \mathbf{U}+O(\Delta \mathbf{U})^{2}
\end{aligned}
$$

Carrying out the truncation of high order terms, expanding the term $\Delta U$ and rearranging them, it is obtained the approximation to $U^{n+1}$ given by the equation 3.53.

$$
\mathbf{U}^{n+1}=\mathbf{U}^{n}-\left(\frac{\partial \mathbf{R}}{\partial \mathbf{U}}\right)_{\mathbf{U}^{n}}^{-1} \mathbf{R}\left(\mathbf{U}^{n}\right)
$$

Where the term $\partial \mathbf{R} / \partial \mathbf{U}$ is called Jacobian matrix represented by the symbol $J$, with the introduction of the damping factor $\gamma$, it is obtained the final form of Newton's method (equation $3.54)$.

$$
\mathbf{U}^{n+1}=\mathbf{U}^{n}-\gamma J^{-1}\left(\mathbf{U}^{n}\right) \mathbf{R}\left(\mathbf{U}^{n}\right)
$$


With the Jacobian given by the matrix 3.55.

$$
J=\left[\begin{array}{lll}
\partial \mathbf{R}_{1} / \partial \mathbf{u}_{1} & \partial \mathbf{R}_{1} / \partial \mathbf{u}_{2} & \partial \mathbf{R}_{1} / \partial \mathbf{p} \\
\partial \mathbf{R}_{2} / \partial \mathbf{u}_{1} & \partial \mathbf{R}_{2} / \partial \mathbf{u}_{2} & \partial \mathbf{R}_{2} / \partial \mathbf{p} \\
\partial \mathbf{R}_{3} / \partial \mathbf{u}_{1} & \partial \mathbf{R}_{3} / \partial \mathbf{u}_{1} & \partial \mathbf{R}_{1} / \partial \mathbf{p}
\end{array}\right]
$$

And its terms by the equations 3.56 to 3.64 .

$$
\begin{gathered}
\frac{\partial \mathbf{R}_{1}}{\partial \mathbf{u}_{1}}=\mathbf{C}_{1}\left(\mathbf{u}_{1}\right)+\mathbf{C}_{1}(1) \mathbf{u}_{1}+\mathbf{C}_{2}\left(\mathbf{u}_{2}\right)+2 \mathbf{K}_{11}+\mathbf{K}_{22} \\
\frac{\partial \mathbf{R}_{1}}{\partial \mathbf{u}_{2}}=\mathbf{C}_{2}(1) \mathbf{u}_{1} \\
\frac{\partial \mathbf{R}_{1}}{\partial \mathbf{p}}=-\mathbf{Q}_{1} \\
\frac{\partial \mathbf{R}_{2}}{\partial \mathbf{u}_{1}}=\mathbf{C}_{1}(1) \mathbf{u}_{2}+\mathbf{K}_{21} \\
\frac{\partial \mathbf{R}_{2}}{\partial \mathbf{u}_{2}}=\mathbf{C}_{1}\left(\mathbf{u}_{1}\right)+\mathbf{C}_{2}\left(\mathbf{u}_{2}\right)+\mathbf{C}_{2}(1) \mathbf{u}_{2}+\mathbf{K}_{11}+2 \mathbf{K}_{22} \\
\frac{\partial \mathbf{R}_{2}}{\partial \mathbf{p}}=-\mathbf{Q}_{2} \\
\frac{\partial \mathbf{R}_{3}}{\partial \mathbf{u}_{1}}=-\mathbf{Q}_{1}^{T} \\
\frac{\partial \mathbf{p}}{2}=0 \\
\\
\frac{\mathbf{R}_{2}}{\mathbf{R}_{2}} \\
\end{gathered}
$$

The resulting system was solved using the multifrontal massively parallel sparse direct solver (MUMPS) (AMESTOY et al., 2000). 


\section{GEOMETRY AND MESH}

\subsection{Geometry}

Overall, three domains were constructed, all of them were divided in two regions. The hatched regions (or design domain), were topology optimization was able to change the solid and void distribution. And the no hatched regions, were only the NSE were solved without the influence of topology optimization. The flow was able to flow freely between the interfaces of the of hatched and no hatched regions and all boundary conditions were applied on the outer geometry contours.

The first was chosen so that the simulation results obtained using the proposed optimization methodology can be compered with the work of Andreasen (2017). Figure 20 depicts the first geometry (or geometry 1).

Figure 20 - First geometry with 2D planar domain.

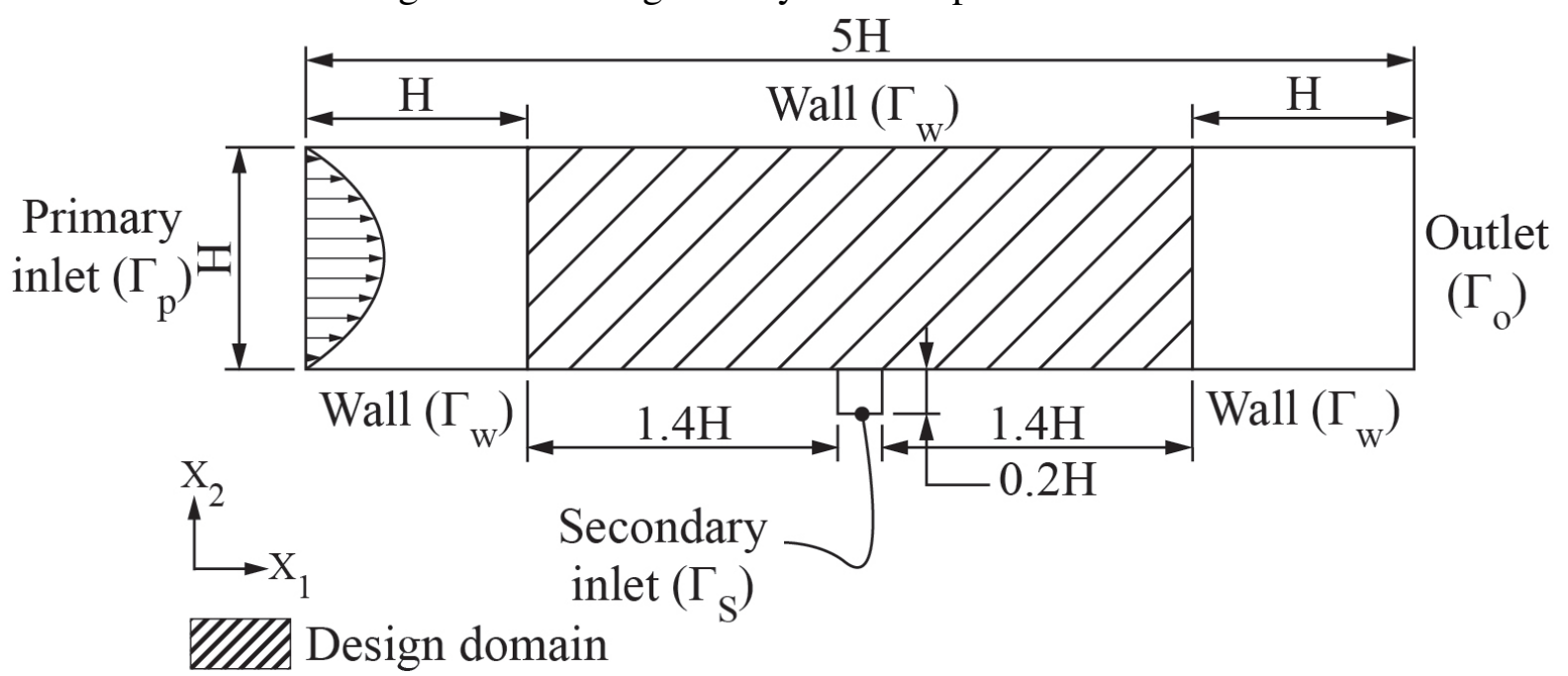

Source: Author.

For the inlet boundary condition, the velocity was imposed by the profile given in the equation 4.1 .

$$
V(y)=\frac{6 y(H-y) \bar{U}}{H^{2}}
$$

where $\bar{U}$ is the averaged velocity at inlet region. And for the secondary inlet $\left(\Gamma_{\mathrm{p}}\right)$ and outlet $\left(\Gamma_{\mathrm{O}}\right)$ regions, the stress free boundary conditions was set up. This means that the natural boundary condition (right hand side of equation 3.33) was forced to be equal to zero. The 
equations 4.2 and 5.1 show the expanded natural boundary condition (over $i$ and $j$ of index notation)

- Secondary inlet:

$$
\oint_{\Gamma_{S}}\left[-p+2 \mu\left(\frac{\partial u_{2}}{\partial x_{2}}\right)\right] w_{2} d \Gamma=0
$$

- Outlet:

$$
\oint_{\Gamma_{O}}\left[-p+2 \mu\left(\frac{\partial u_{1}}{\partial x_{1}}\right)\right] w_{1} d \Gamma=0
$$

Where $u_{1}$ and $u_{2}$ are, respectively, the velocity in $x_{1}$ and $x_{2}$ Cartesian direction and $w_{1}$ and $w_{2}$ are, respectively, the weight function of $u_{1}$ and $u_{2}$ velocity components. With the imposition of this kind of boundary conditions, the pressure, at the same boundary, is implicitly constrained by (REDDY; GARTLING, 2010).

$$
\overbrace{p=2 \mu \frac{\partial u_{1}}{\partial x_{1}}}^{\text {at } \Gamma_{\mathrm{O}}} \text { and } \overbrace{p=2 \mu \frac{\partial u_{2}}{\partial x_{2}}}^{\text {at }}
$$

Once, the only way to make the natural term of weak form of momentum equation 3.33 goes to zero, is to ensure that the terms inside the brackets goes to zero.

The second geometry, geometry 2 or radial secondary inlet (RSI) geometry was constructed similarly to the first. This way, the modification in the domain was gradually introduced. However, the axisymmetric boundary condition was inserted, and the secondary inlet enlarged (making the proportions between the primary and secondary inlet closer to a jet pump), as shown by the Figure 21.

For this geometry, the imposed boundary conditions at inlet was,

$$
V(r)=2 \bar{U}\left(1-\frac{r^{2}}{R^{2}}\right)
$$

Where the $R$ is the radius of the primary inlet. And for the secondary inlet $\left(\Gamma_{\mathrm{p}}\right)$ and outlet $\left(\Gamma_{\mathrm{O}}\right)$ regions, the stress free boundary was imposed

- Secondary inlet:

$$
\oint_{\Gamma_{S}}\left[-p+2 \mu\left(\frac{\partial u_{r}}{\partial r}\right)\right] w_{r} d \Gamma=0
$$

- Outlet:

$$
\oint_{\Gamma_{O}}\left[-p+2 \mu\left(\frac{\partial u_{z}}{\partial z}\right)\right] w_{z} d \Gamma=0
$$


Figure 21 - Second geometry with 2D axisymmetric domain with radial secondary inlet (RSI).

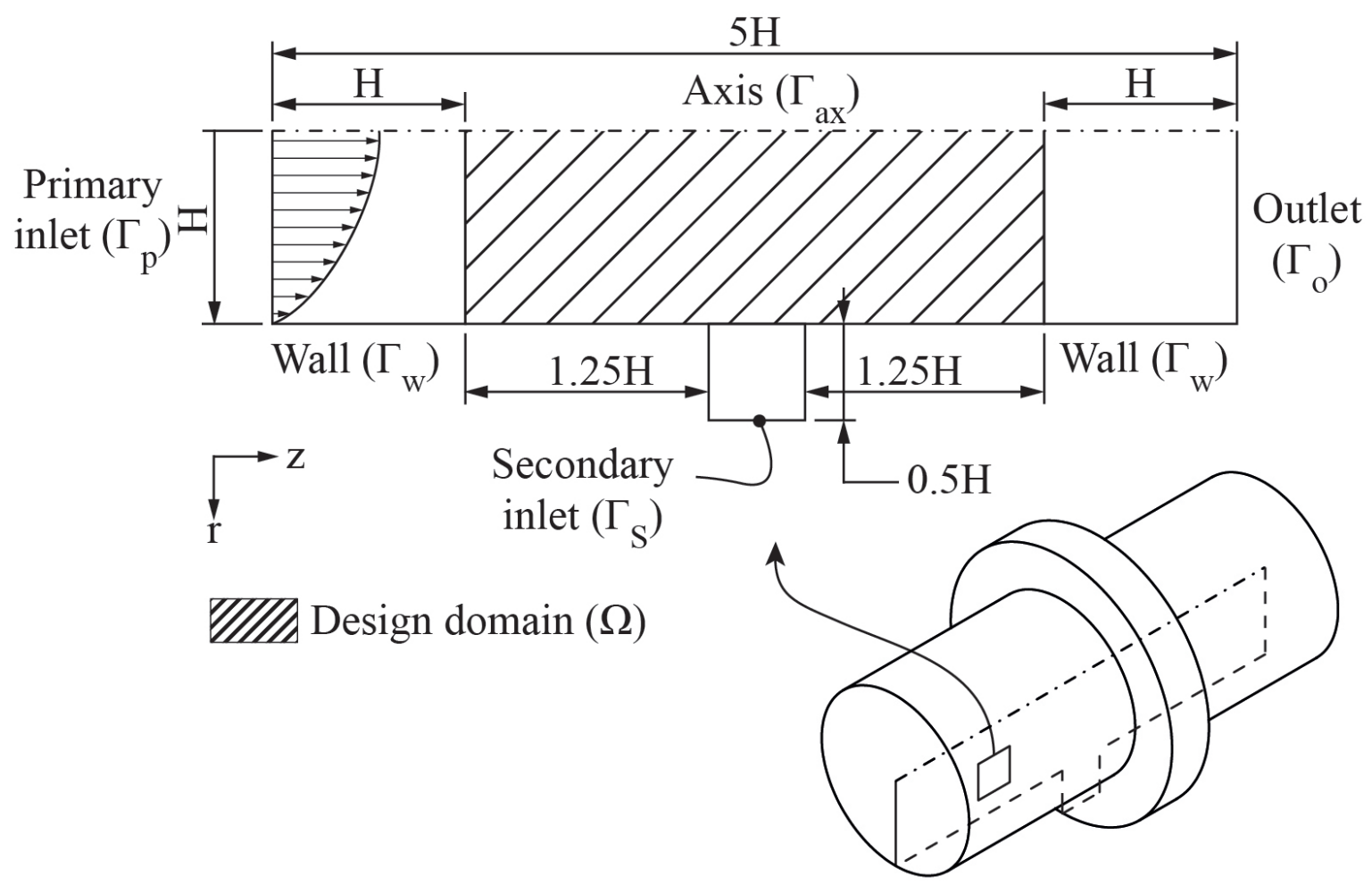

Source: Author.

And the implicit pressure constraint

$$
\overbrace{p=2 \mu \frac{\partial u_{z}}{\partial x_{z}}}^{\text {at }} \text { and } \overbrace{p=2 \mu \frac{\partial u_{r}}{\partial x_{r}}}^{\Gamma_{\mathrm{O}}}
$$

Finally, the third and last domain, geometry 3 or axial secondary inlet (ASI) geometry was built with its boundaries at the same position that a conventional jet pump as depicted by Figure 22.

The boundary condition set up to the inlet region of geometry 3 was given by the velocity profile

$$
V(r)=2 \bar{U}\left(1-\frac{r^{2}}{R^{2}}\right)
$$

And for the secondary inlet $\left(\Gamma_{\mathrm{p}}\right)$ and outlet $\left(\Gamma_{\mathrm{O}}\right)$ regions, the stress free boundary was imposed

- Secondary inlet:

$$
\oint_{\Gamma_{S}}\left[-p+2 \mu\left(\frac{\partial u_{z}}{\partial z}\right)\right] w_{z} d \Gamma=0
$$


Figure 22 - Third geometry with 2D axisymmetric domain with axial secondary inlet (ASI).

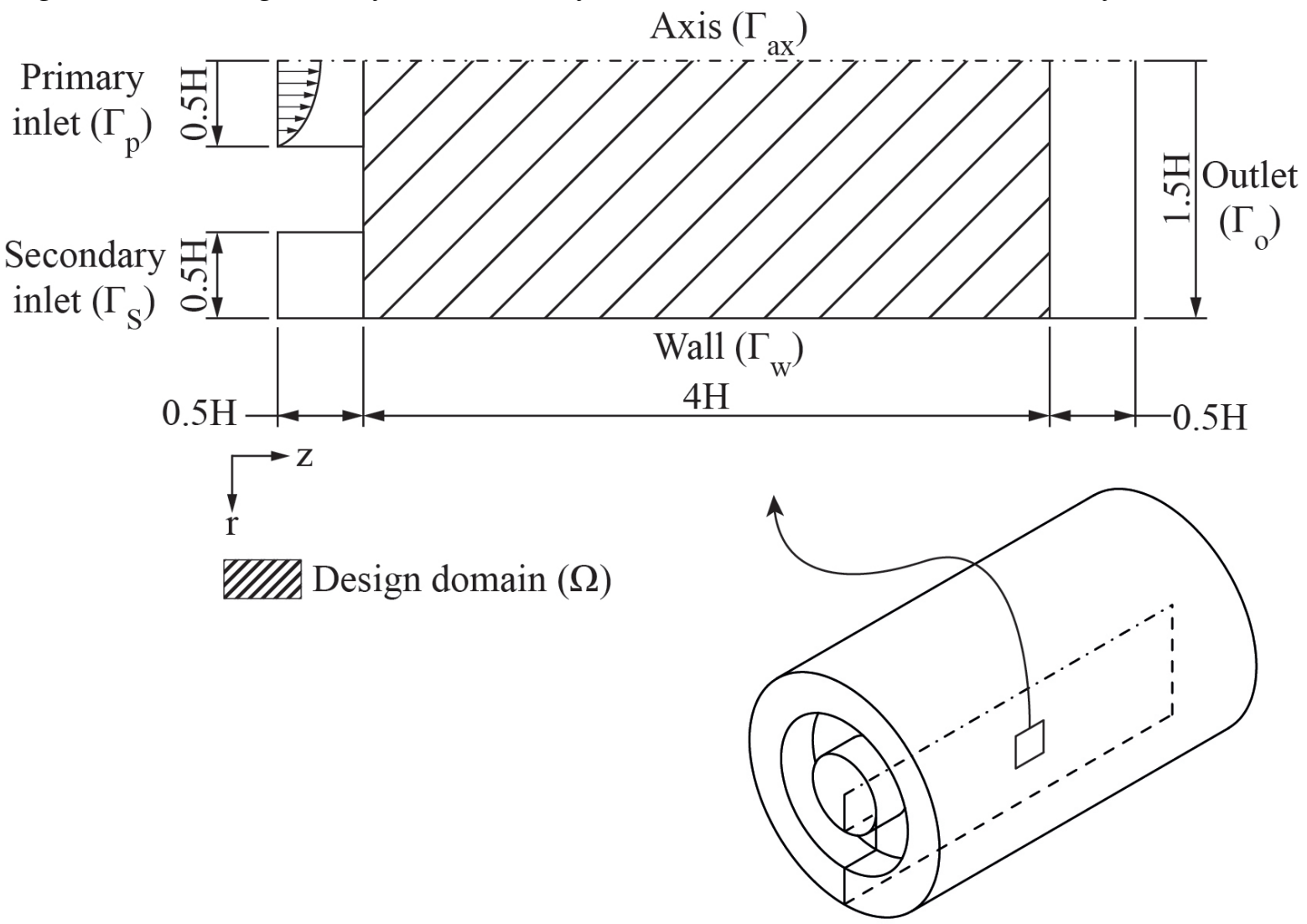

Source: Author.

- Outlet:

$$
\oint_{\Gamma_{O}}\left[-p+2 \mu\left(\frac{\partial u_{z}}{\partial z}\right)\right] w_{z} d \Gamma=0
$$

And the implicit pressure constraint

$$
\overbrace{p=2 \mu \frac{\partial u_{z}}{\partial x_{z}}}^{\text {at }} \Gamma_{0} \quad \text { and } \overbrace{p=2 \mu \frac{\partial u_{z}}{\partial x_{z}}}^{\text {at }}
$$

The $\bar{U}$ present in the velocity profile equations was calculated based on the dimensionless Reynolds number, that in the case of the study, is given by the equation 4.13.

$$
R e=\frac{\rho \bar{U} H a}{\mu}
$$

The parameter $a$ in the equation 4.13 was inserted to adjust the characteristic length $H$ in the cases like in the Figure 22 that the inlet dimension is smaller than the $H$. Thus, its values are equal to one in the first two domains and 0.5 in the last domain. The reference pressure of the simulations was of $101325 \mathrm{~Pa}$, in such way that the gauge pressure was obtained in the end 
of simulations.

\subsection{Mesh}

The mesh selected for the simulation was unstructured with two different values of element density. The region with smaller elements was located where the topology optimization was carried out (hatched in Figures 20 to 22), with triangles elements with maximum element size (the length of the longest edge of the element (COMSOL, 2019)) equal to $H / 100$. The remaining domain (extensions built to ensure that the boundary conditions do not interfere in the optimization) was filled with coarser triangular elements with their maximum size equal to $H / 10$ (a discussion about the mesh convergence was made in appendix D). A constant growth ratio smoothed the transition between the two regions, and all elements were connected by its nodes; thus, there is no need to control the flux between the cells. Figure 23 presets the mesh density distribution to geometry 1 , being that the same distribution and mesh behaviour was observed in the other geometries.

Figure 23 - Colored map of the element size distribution on the geometry 1.

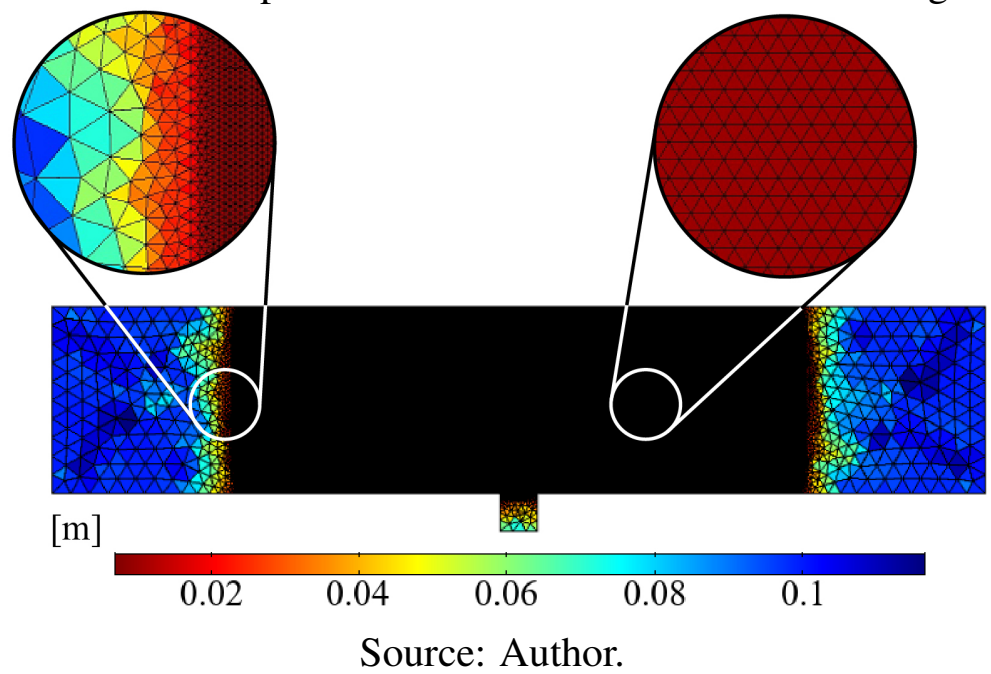

The value of the characteristic length $\mathrm{H}$ was chosen equal to $1 \mathrm{~m}$. Therefore, the number of elements (hatched area in parentheses) of geometries 1,2 and 3 were, respectively, 77359 (74512), 79668 (37648) and 153760 (149018). 


\section{OPTIMIZATION PROBLEM}

Mathematical model For the optimization process, it is essential to define the objective problem. The definition must indicate the objective function and its constraint and if the goal of the optimization is the maximization or minimization of the given objective function. The following definition was found considering the problem described in this work,

$$
\text { Minimize : } \quad J_{i}(N(\breve{\xi}), \breve{\xi}) \text { for } i=1,2,3
$$

$$
\begin{array}{ll}
\text { Subjected to: } & \text { 1) } \\
& g(p) \leq 0 \\
\text { 2) } & 0 \leq \breve{\xi} \leq 1
\end{array}
$$

The three objective functions (and constraints) selected for the optimization were taken from the work done by Andreasen (2017). The first measures the pressure force at the secondary inlet $\left(J_{1}\right)$ :

$$
J_{1}=\oint_{\Gamma_{S}} p d \Gamma
$$

The second objective function $\left(J_{2}\right)$ measures the flow rate that enters the domain crossing the secondary inlet boundary. It means that $J_{2}$ objective function sine is dependent on the scalar product between the velocity vector and the secondary inlet normal vector, which can be negative or positive depending on the direction of these vectors since each geometry has a different position of secondary inlet or coordinate frame. This behaviour directly affects the definition of the nested problem 5.1, which would need to change the objective of minimization to maximization, or vice versa, to match with the objective of increase the flow rate towards device interior. Alternatively, it was decided to change the signal of the objective $J_{2}$ (as a function of the geometry) to keep the goal of minimization. To this end, the variable $\kappa$ (assuming values of -1 or 1 ) was introduced in the $J_{2}$ to keep the condition described above. Table 2 summarizes the values of $\kappa$ used for each geometry.

Where the flow rate at secondary inlet region that enters the domain is given by

$$
J_{2}=\kappa \oint_{\Gamma_{S}} u_{n} d \Gamma
$$

The third objective function $\left(J_{3}\right)$ measures the flow rate that leaves the domain crossing the 
Table $2-\kappa$ variable values with respect to the geometry for $J_{2}$ objective function.

\begin{tabular}{cc}
\hline Geometry & $\kappa$ \\
\hline Type 1 - Figure 20 & -1 \\
\hline Type 2 - Figure 21 & 1 \\
\hline Type 3 - Figure 22 & -1 \\
\hline
\end{tabular}

Source: Author.

outlet given by

$$
J_{3}=-\oint_{\Gamma_{0}} u_{n} d \Gamma
$$

The minus sign was inserted to adjust the objective function with the optimization goal.

The constraint $g(p)$ controls the static pressure loss inside the domain assessing the ratio of the static pressure integral at inlet in each optimization step $(p)$ by a reference value $\left(p_{r e f}\right)$ times the parameter $\sigma$. The reference value is the pressure obtained by carrying out simulations without the optimization routine, in such way that each geometry has its own $p_{\text {ref }}$. The variable $\sigma$ is a arbitrary positive real number that works as a tuning parameter, controlling how much the optimized domain can demand a higher pressure at the inlet than the reference domain. Exemplifying, if a value of 50 is chosen to $\sigma$, the constraint admits that the pressure integral at the primary inlet during the optimization $(p)$ is, at most, equal to 50 times higher than the pressure integral at primary inlet of same geometry without optimization routine $\left(p_{\text {ref }}\right)$.

$$
g(p) \equiv \frac{\oint_{\prod_{P}} p d \Gamma}{\sigma \oint_{\Gamma_{P}} p_{r e f} d \Gamma}-1
$$

Also, this constraint indirectly controls the amount of mass inside the optimized domain once every solid region interferes in the pressure at the inlet boundary region.

\subsection{Sensitivity evaluation}

As presented in the introduction section, the discrete adjoint method was chosen to calculate the objective function gradients with respect to the design variables. To this end, given a generic objective function (equation 5.6). 
Where $\mathbf{U}$ is the vector that contains all the flow variables. The objective function variables must respect the discretized Navier-Stokes equation represented by equation 5.7.

$$
\mathbf{N}(\mathbf{U}(\boldsymbol{\xi}), \boldsymbol{\xi})=0
$$

Taking place the derivative of the objective function (Eq. 5.6) with respect to the design variables it is obtained the desired gradient:

$$
\frac{d \mathbf{J}}{d \boldsymbol{\xi}}=\frac{\partial \mathbf{J}}{\partial \mathbf{U}} \frac{d \mathbf{U}}{d \boldsymbol{\xi}}+\frac{\partial \mathbf{J}}{\partial \boldsymbol{\xi}}
$$

Where the term $\mathrm{d} \mathbf{U} / \mathrm{d} \boldsymbol{\xi}$ can be obtained by the linearized NSE (equation 5.9), such that:

$$
\frac{\partial \mathbf{N}}{\partial \mathbf{U}} \frac{d \mathbf{U}}{d \boldsymbol{\xi}}+\frac{\partial \mathbf{N}}{\partial \boldsymbol{\xi}}=0
$$

Where the term $\partial \mathbf{N} / \partial \mathbf{U}$ is the linearization of the flow residual with respect to the flow variables and $\partial \mathbf{N} / \partial \boldsymbol{\xi}$ is the linearization of the flow residual with respect to the design variables. This way, defining:

$$
\begin{array}{cc}
\mathbf{z} \equiv d \mathbf{U} / d \boldsymbol{\xi} & \mathbf{A} \equiv \partial \mathbf{N} / \partial \mathbf{U} \\
\mathbf{e}^{T} \equiv \partial \mathbf{J} / \partial \mathbf{U} & \mathbf{f} \equiv-\partial \mathbf{N} / \partial \boldsymbol{\xi}
\end{array}
$$

It is possible to write,

$$
\frac{d \mathbf{J}}{d \boldsymbol{\xi}}=\mathbf{e}^{T} \mathbf{z}+\frac{\partial \mathbf{J}}{\partial \boldsymbol{\xi}}
$$

With,

$$
\mathbf{A z}=\mathbf{f}
$$

To the adjoint approach is stated,

$$
\mathbf{A}^{T} \mathbf{b}=\mathbf{e}
$$

Where $\mathbf{b}$ are the adjoint variables that satisfy the linear system 5.13. This way, the first term 
of the right-hand side of equation 5.11 can be replaced by,

$$
\begin{aligned}
& \mathbf{f}=\mathbf{A z} \\
& \mathbf{b}^{T} \mathbf{f}=\mathbf{b}^{T} \mathbf{A z} \\
& \mathbf{b}^{T} \mathbf{f}=\left(\mathbf{A}^{T} \mathbf{b}\right)^{T} \mathbf{z} \\
& \mathbf{b}^{T} \mathbf{f}=\mathbf{e}^{T} \mathbf{z}
\end{aligned}
$$

Solving the equation 5.11, the computational cost increases as a function of the number of design variables because each of them will have its term $\mathbf{f}$. The utilization of such an approach receives the name of direct differentiation (DD). Alternatively, it is possible to calculate only one time the equivalent adjoint problem using the relation 5.14. This way, because each problem has only one $\mathbf{e}$, the computational cost does not increase in function of the design variables.

The development of this section was based in the introduction work done by Giles \& Pierce (2000).

\subsection{Optimization algorithm}

Among the algorithms available to conduct the topology optimization, the method of moving asymptotes (SVANBERG, 1987) (MMA) is frequently used in the literature (DEHGHANI et al., 2020; SUN; LIEBERSBACH; QIAN, 2020). This method is especially attractive because the typical set up of a topology optimization problem matches the recommendations for its use. The evaluation of the gradients is much more expensive than the solution of a subproblem involving nonlinear optimization, and its design vector has a large number of variables with few constraints (HÖRNLEIN; SCHNITTKOWSKI, 2013). Moreover, the variation of the MMA method, the globally converged MMA (GCMMA) (SVANBERG, 2002), is already implemented in the COMSOL Multiphysics ${ }^{\circledR}$ software.

The MMA method needs to be presented before the GCMMA method. The reason for that 
will be clear at the end of this section. Given a generic problem of optimization 5.15,

$P$ : Minimize

$$
f_{0}(\mathbf{x})
$$

Subject to

$$
\begin{array}{cc}
f_{i} \leqslant \hat{f}_{i} & \text { for } i=1, \ldots, m \\
x_{\text {min }} \leqslant x_{j} \leqslant x_{\text {max }} & \text { for } j=1, \ldots, n
\end{array}
$$

Where $\mathbf{x}$ is the vector of design variables. Svanberg (1987) summarizes in four steps the MMA method.

1. Choose a starting value of the design variables.

2. Evaluate the objective function, the restrictions and the gradients for all $i=0,1, \ldots, m$.

3. Create a convex subproblem $P^{k}$ replacing the function $f_{i}$ by an explicit function $f_{i}^{(k)}$, using the gradients calculated in step 1.

4. Solve the subproblem, then set this solution as the new values of design variables. Return to step 2 until the convergence criteria are obtained.

Where the subproblem in the MMA method is defined by first-order approximation $P^{k}:$ Minimize

$$
\sum_{j=1}^{n}\left(\frac{p_{0 j}^{k}}{U_{j}^{(k)}-x_{j}}+\frac{q_{0 j}^{k}}{x_{j}-L_{j}^{(k)}}\right)+r_{0}^{(k)}
$$

Subject to

$$
\begin{aligned}
& \sum_{j=1}^{n}\left(\frac{p_{i j}^{k}}{U_{j}^{(k)}-x_{j}}+\frac{q_{i j}^{k}}{x_{j}-L_{j}^{(k)}}\right)+r_{i}^{(k)} \leqslant \hat{f_{i}} \quad \text { for } i=1, \ldots, m \\
& \max \left(x_{\min }, \alpha_{j}^{(k)}\right) \leqslant x_{j} \leqslant \min \left(x_{\max }, \beta_{j}^{(k)}\right) \text { for } j=1, \ldots, n
\end{aligned}
$$

With,

$$
\begin{gathered}
p_{i j}(k)=\left\{\begin{array}{cc}
\left(U_{j}^{(k)}-x_{j}^{(k)}\right)^{2} \partial f_{i} / \partial x_{j}, & \text { if } \partial f_{i} / \partial x_{j}>0 \\
0, & \text { if } \partial f_{i} / \partial x_{j} \leqslant 0
\end{array}\right. \\
q_{i j}(k)=\left\{\begin{array}{cc}
0, & \text { if } \partial f_{i} / \partial x_{j} \geqslant 0 \\
-\left(x_{j}^{(k)}-L_{j}^{(k)}\right)^{2} \partial f_{i} / \partial x_{j}, & \text { if } \partial f_{i} / \partial x_{j}<0
\end{array}\right. \\
r_{i}^{(k)}=f_{i}\left(\mathbf{x}^{(k)}\right)-\sum_{j=1}^{n}\left(\frac{p_{i j}^{(k)}}{\left(U_{j}^{(k)}-x_{j}^{(k)}\right)}+\frac{q_{i j}^{(k)}}{\left(x_{j}^{(k)}-L_{j}^{(k)}\right)}\right)
\end{gathered}
$$


Where $\alpha_{j}^{(k)}$ and $\beta_{j}^{(k)}$ are moving parameters introduced in the problem to avoid problems during the calculation of dividing by zero.

$$
\frac{\partial^{2} f_{i}^{(k)}}{\partial x_{j}^{2}}=\frac{2 p_{i j}^{(k)}}{\left(U_{j}^{(k)}-x_{j}\right)^{3}}+\frac{2 q_{i j}^{(k)}}{\left(x_{j}-L_{j}^{(k)}\right)^{3}}
$$

And, if $i \neq j$

$$
\frac{\partial^{2} f_{i}^{(k)}}{\partial x_{j} \partial x_{i}}=0
$$

From equation 5.20, it is clear to see that the parameters $U_{j}^{(k)}$ and $L_{j}^{(k)}$ (also called asymptotes) can change the value of the second derivative. With values of the asymptotes parameters closer to the value of $x_{j}$, the larger the second derivative becomes. As a consequence, more curvature is given to the equation $f_{i}^{(k)}$ and more conservative is the subproblem. Conversely, if the value of the asymptotes parameters away from the $x_{j}$, smaller will be the second derivative. Therefore, less curvature and less conservative is the subproblem. In general, the values of $U_{j}^{(k)}$ and $L_{j}^{(k)}$ can be changed between the iteration. For example, (i) if the solutions are oscillating, the stabilization can be created by moving the asymptotes closer to the current design point and (ii) if the solution is converging too slowly, the process can be accelerated by moving the asymptotes away from the current design point. Because of this behaviour, these parameters receive the name of moving asymptotes that name the method. The choice of the $U_{j}^{(k)}$ and $L_{j}^{(k)}$ must satisfy the requirements 5.22 .

$$
L_{j}^{(k)}<x_{j}^{(k)}<U_{j}^{(k)}
$$

However, Svanberg (1987) highlights that there is not a rule to choose the values of the $U_{j}^{(k)}$ and $L_{j}^{(k)}$ as well as for the parameters $\alpha_{j}^{(k)}$ and $\beta_{j}^{(k)}$. This way, each code and problem can have its own set of parameters. Therefore, as it was not possible to access the COMSOL Multiphysics ${ }^{\circledR}$ source code, the values of these parameters are not indicated here.

The GCMMA method executes the MMA method (outer iteration) as described above. However, another step is introduced. The solution of the subproblem $\mathbf{x}$ needs to satisfy the condition given by the inequality 5.23 .

$$
f_{i}^{(k, v)}\left(\mathbf{x}^{(\mathbf{k}, \mathbf{v})}\right) \geqslant f_{i}\left(\mathbf{x}^{(\mathbf{k}, \mathbf{v})}\right) \quad \text { for all } i
$$

If these conditions are not satisfied, another inner iteration $v$, more conservative, is evaluated 
and solved for those design variables that violate the inequality. The results are then tested again and, if the new values satisfy the restriction, the next steps of MMA (described above) are conducted. Otherwise, another inner iteration takes place. The process continues until the convergence is achieved. The additional superscript term indicates the inner iteration step.

The utilization of GCMMA is preferred rather than MMA because the last one can not guarantee convergence, which, in practice, leads to unsatisfactory results (ZILLOBER, 1993; SVANBERG, 2002). Hereafter, the word "iteration" will refer to the outer optimization iteration (see Figure 25). Other iterations will be specified if needed.

\subsection{Optimization strategy}

The central core of the optimization strategy was based on the work of Andreasen (2017). In his work, the optimization used a continuation technique to the variable $\beta$, which controls how sharp is the transition between the void and solid region in the hyperbolic filter (equation 3.47). In each topology optimization simulation, the $\beta$ variable was modified starting from 1 and doubles until it reaches the value of 64, where a group of iteration (Figure 25) with a certain value of $\beta$ is called a step (in total, 7 steps). A step ends or if the maximum number of iteration of optimization algorithm is reached or if a certain criteria is reached. In Andreasen (2017), a step ends or if the total number of MMA algorithm iterations reaches 1000 (40 iterations in the

first six steps and, in the last step, iterations 760) or in the case of $\left\|\breve{\xi}^{\left(k_{o}\right)}-\breve{\xi}^{\left(k_{o}-1\right)}\right\|<0.01$, where $k_{o}$ is the iteration counter.

Herein, two modifications were made in the steps. The first one was the utilization of the same number of optimization in each step. And the other one, the increase in the number of iteration in each step. To determine the best number of iterations, simulations was carried out using $J_{1}$ objective function and modifying the maximum number of iteration in each step assuming the values of 40, 200, 400 and 600 GCMMA iterations, counting inner (limited to 10) and outer, for the case of geometry 1, the results were plotted in Figure 25.

One can observe that between the 400 to 600 iterations, the difference between the solid distribution was small enough to infer that the convergence was reached; hence, the 600 iteration was used. Moreover, the second criteria to the step ends was set up to if the relative difference between two consecutive objective function evaluation be less than $0.1 \%$. It is important to say that, despite of the difference of in the optimization algorithm used and in the termination 
Figure 24 - Final solid distribution for 50, 200, 400 and 600 iteration in each step.
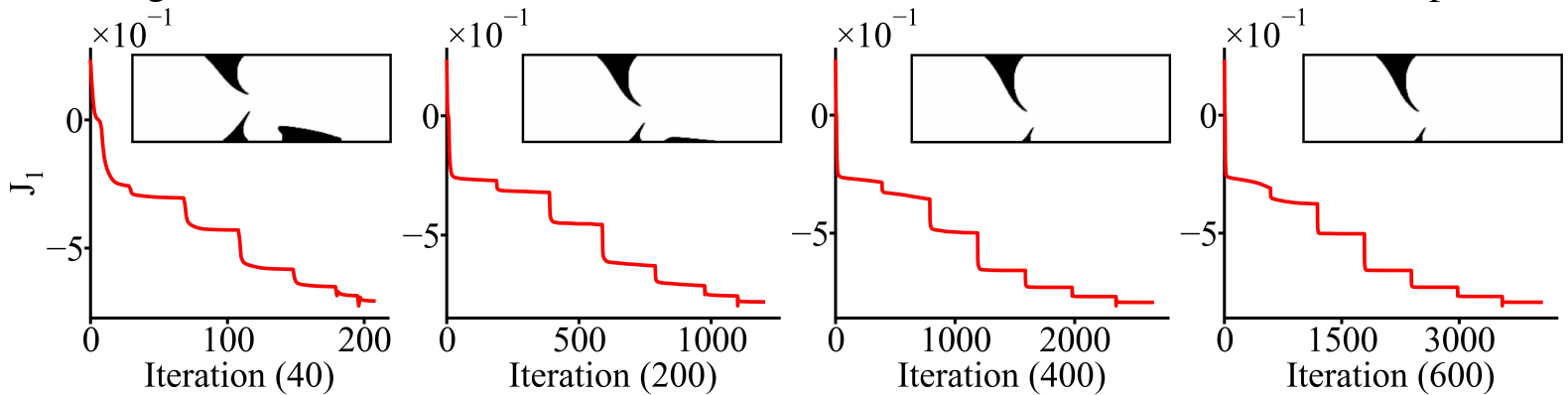

Source: Author.

criteria, the same minimum in the optimization problem is expected, once a strong minimum was found. However, if the simulations ran less step than needed, differences are expected (as evidenced by Figure 24).

Additionally, previous simulation pointed that if the number of iterations was further increased, the simulation starts to converge to the minimum associated with the lowest values of $\beta$, which shows to be worst than the minimum of higher $\beta$ and starting with higher values of $\beta$, the simulations are more restrictive and tend to converge to solutions where the secondary inlet was blocked.

As well as in Andreasen (2017), all steps were programmed with filter parameter $r$ equal to 0.0289 and the penalization parameter $q$ equal to $10^{-2}$. 
Figure 25 - Flowchart of the topology optimization routine.

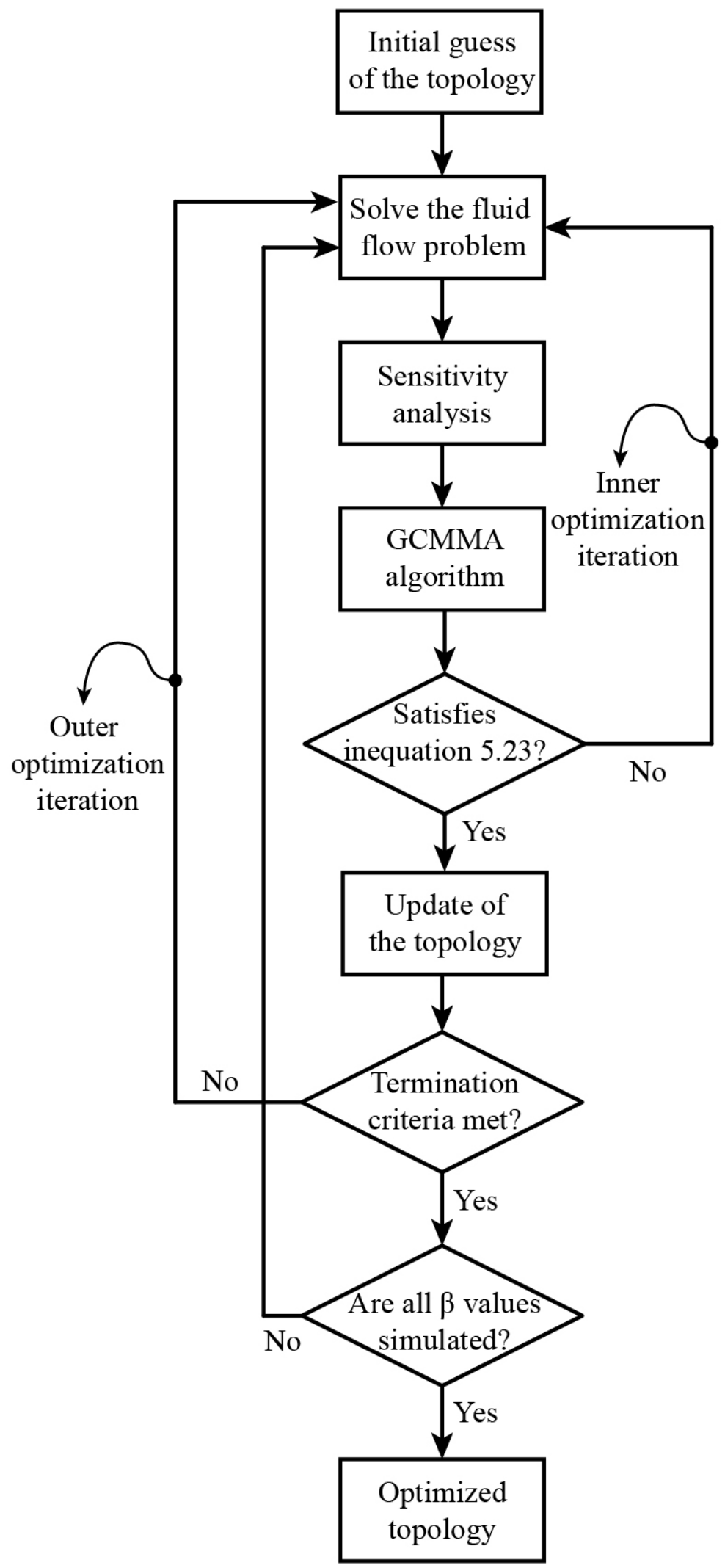

Source: Author. 


\section{RESULTS}

From the methodology chapter 3 , it is possible to conclude that there are extensive possibilities to set up the constraints, flow and geometric parameters. For example, considering only the inlet positions, Reynolds number and loss constraint, if each could assume at least ten values, it is possible to calculate the number of possible combinations using the combinatorial analysis (equation 6.1).

$$
P=10 \cdot 10 \cdot 10=1000
$$

Therefore, as the total number of parameters to be set up and possible values are higher than shown in the example above, it was necessary to consider only a limited range and parameter combinations. Moreover, it was verified that the modules of the software used in this study did not benefit from scalability in parallel computing as depicted by Figure 26.

Figure 26 - Computational time for each optimization iteration as function of the number of computational nodes.

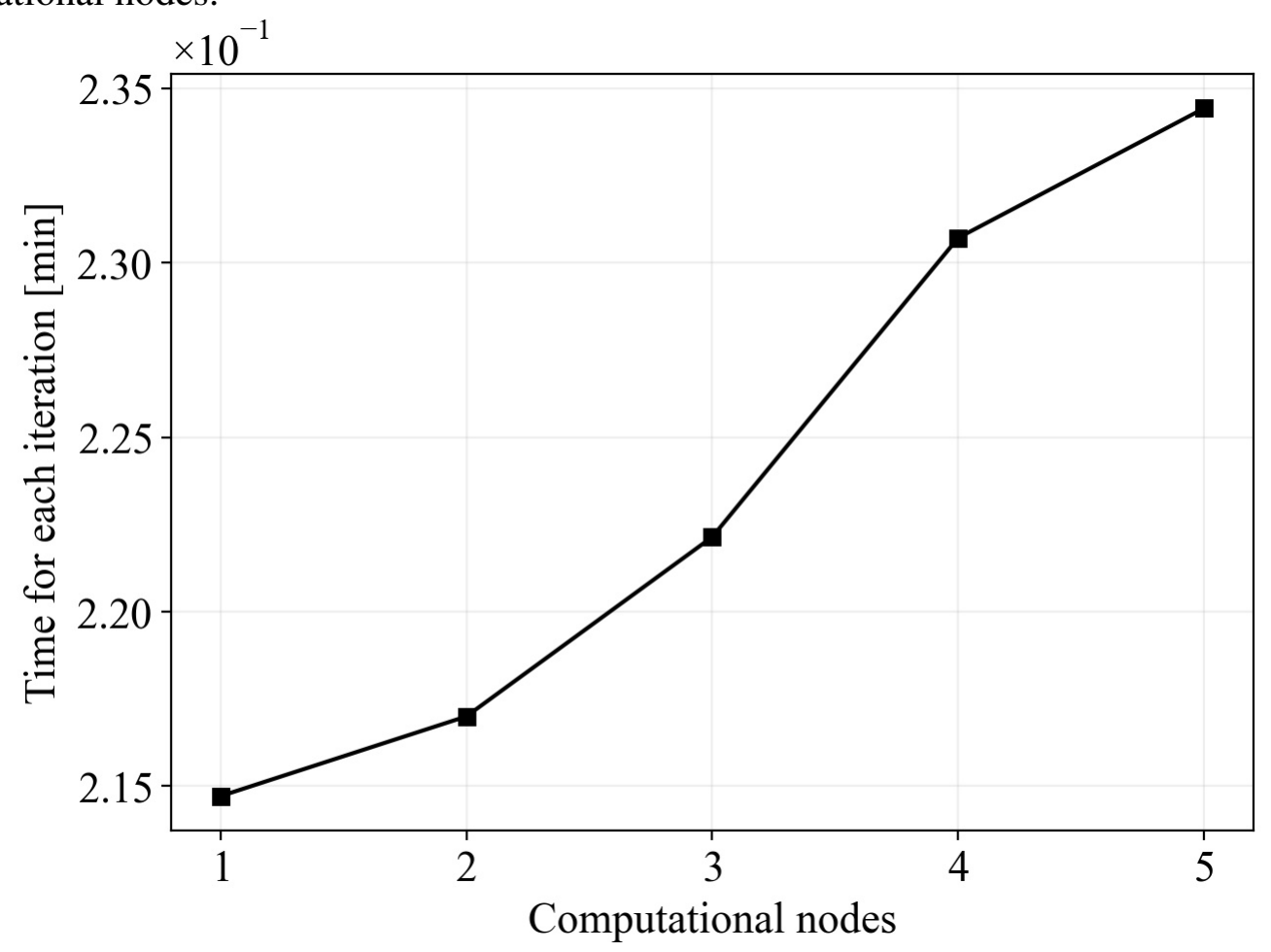

Source: Author.

The test was conducted in the Mintrop cluster of Universidade de São Paulo. Each node has two processors Xeon Gold 6148, with 40 cores and 192 GB of RAM. Such characteristic reinforces the necessity to simulate a reduced number of models. 


\subsection{Comparison procedure}

The first simulation was carried out to compare the result obtained with the methodology proposed in the present work and the results obtained by Andreasen (2017). So, the geometry 1 (Figure 20) was simulated using the same conditions of one case present in his work. The selected case was for Reynolds number equal to 20, sigma equal to 10 and objective function $J_{1}$. As the number of optimization iterations was increased with relation to the number used by Andreasen (2017), the case was simulated twice. One with the same number of optimization iterations and another with the number of optimizations iteration proposed in the current work.

Moreover, it was found that the usual process to run non-dimensional flows, proposed in the user guide of the COMSOL Multiphysics $₫$, presented a convergence problem. This way, differently from the base article, it was used the international system of units and a hypothetical material (with properties described in the methodology section).

The utilization of GCMMA, instead of just MMA (or GCMMA with one inner iteration), was necessary once the utilization of the MMA algorithm also presented a convergence problem (see section 5.2). In both cases, the impossibility of verifying the matrices and optimization algorithm during the evaluations make it impossible to investigate the reason for such behaviours further.

The solid distribution (black regions) and fluid regions (white regions) of present implementation with Andreasen (2017) number of optimization iterations (“A” in Figures 28 and 27), or PIAI, the results extracted from Andreasen (2017) ("B" in Figures 28 and 27), or AR, and present implementation with increased number of optimization iterations ("CC" in Figures 28 and 27), or PIII, were qualitatively compered. When the simulations used the same number of optimization iterations of Andreasen (2017), the optimized topology was closer to that obtained in the base article ("B" in Figures 28 and 27). The main differences were noted in the size and position of the structures, especially the result of the base paper has its jet positioned near the centre of the domain whereas, in the PIAI simulation, the jet was slightly shifted in direction to the secondary inlet. Still, the top structure had its size increased, the bottom decreased, and the right structure, positioned after the secondary inlet, shrunk. 
Figure 27 - Comparison of the pressure field between (A) the results using the present software configuration with the same number of optimization iterations used in the base article, (B) the results of Andreasen (2017) and (C) the result using the number of optimization iteration described in the methodology section.

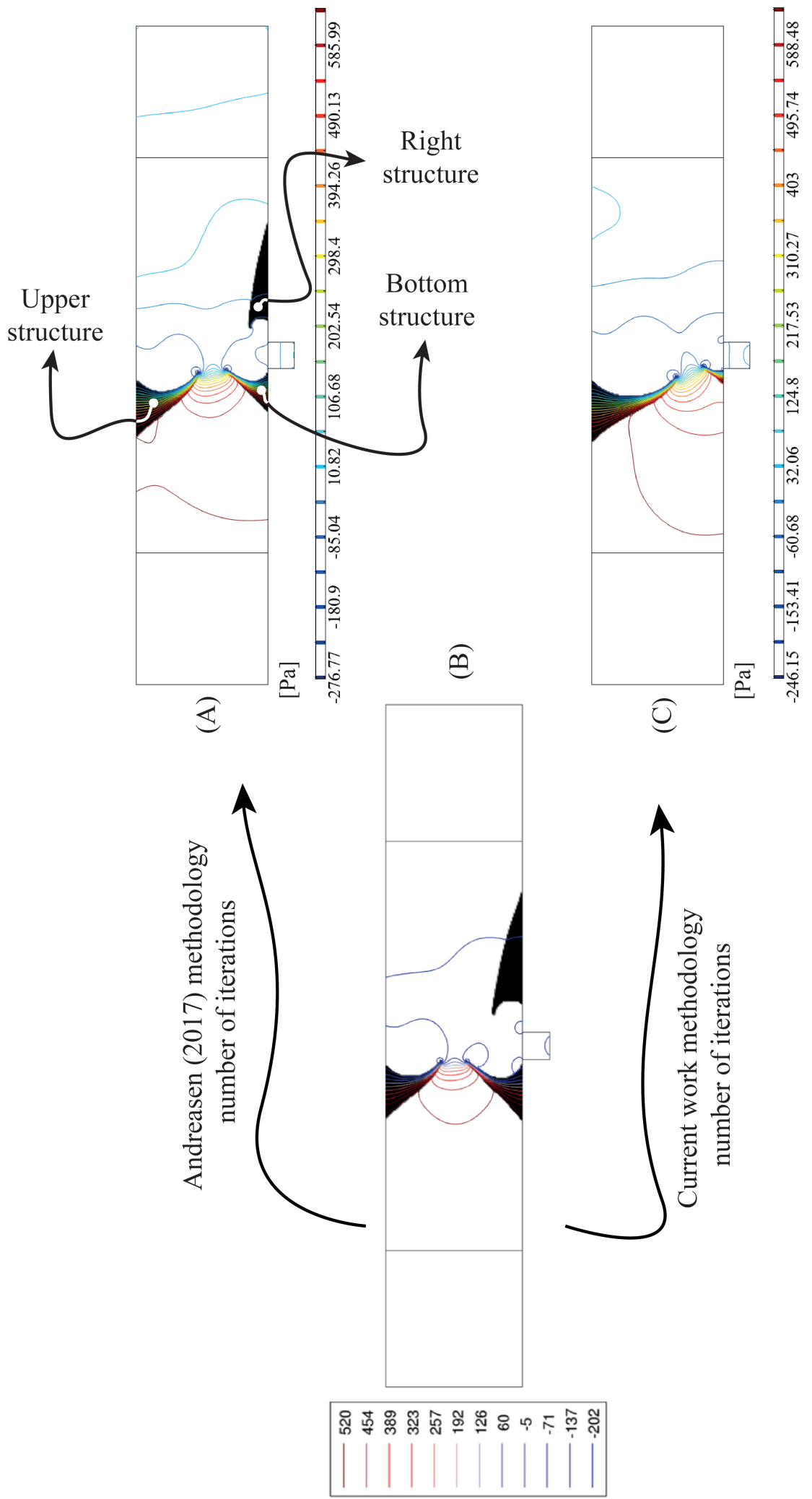

Source: Author. 
Figure 28 - Comparison of the velocity field between (A) the results using the present software configuration with the same number of optimization iterations used in the base article (B) the results of Andreasen (2017) and (C) the result using the number of optimization iterations described in the methodology section.

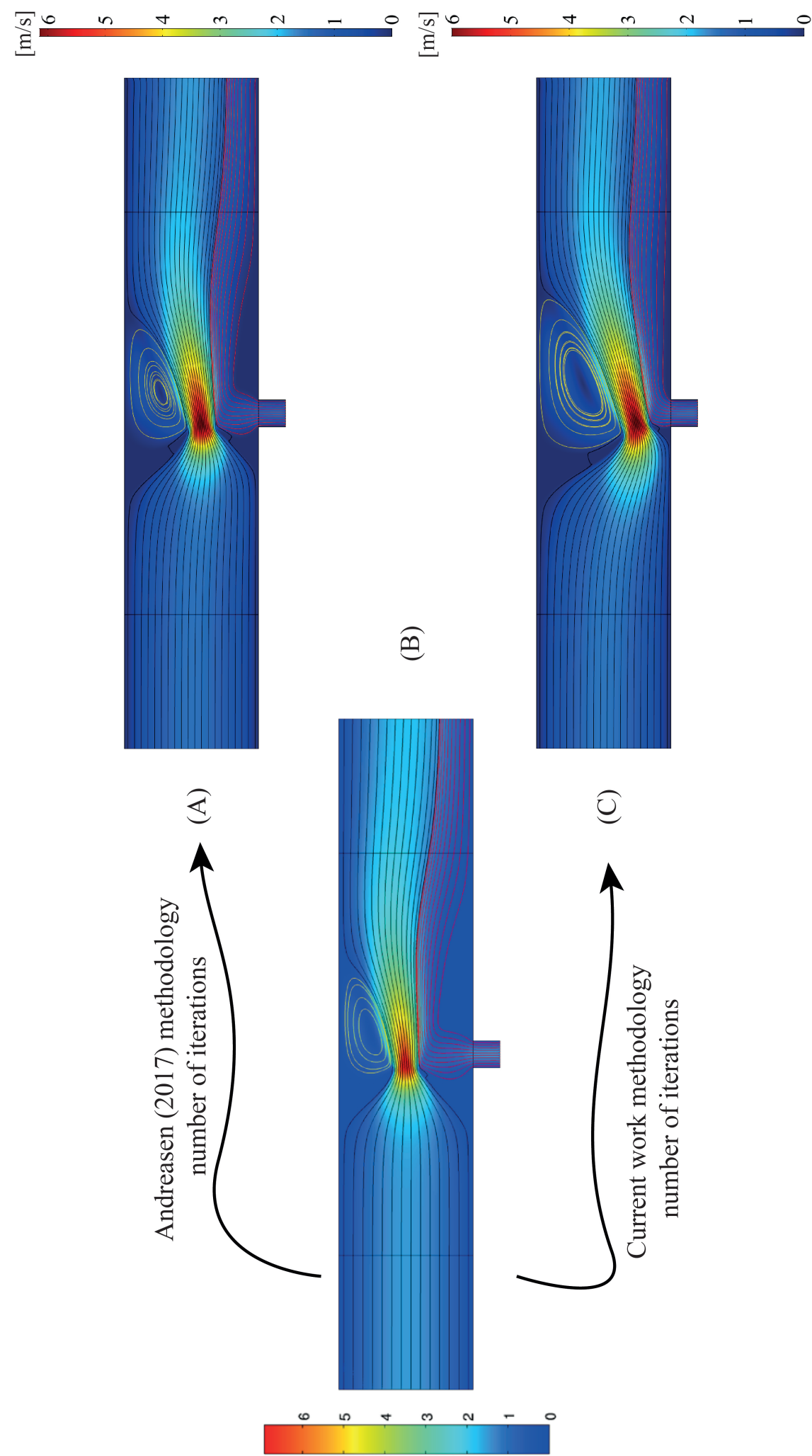

Source: Author. 
The major difference happens in the result obtained with PIII. In this, the upper structure had its size significantly increased, whereas the bottom significantly decreased. The right structure positioned after the secondary inlet at the bottom face, present in results " $A$ " and " $\mathrm{B}$ " in the Figures 28 and 27, disappear and the recirculation zone increases (yellow lines in Figure 28) in both simulations, with emphasis on the geometry that used the current strategy ("C" in Figure 28).

In terms of quantity, the objective function $J_{1}$ of the results " $\mathrm{A}$ " and " $\mathrm{C}$ " were compared. For "A" simulation, $J_{1}$ has the value of $-0.72487 N$ and, for "C" simulation, $-0.79302 N(9.4 \%$ of difference). The efficiency $(\eta)$ was not calculated because geometry 1 can not be considered a jet pump. The simulations conducted in Andreasen (2017) used dimensionless fields of pressure. However, due the lack of information about the denationalization, the comparison between the results was unfeasible.

Therefore, it was possible to infer that the proposed strategy found a better minimum than found by Andreasen (2017) strategy by comparing the results obtained in this dissertation.

\subsection{Objective functions analyses and comparison}

The subsequent simulations were carried out using the last two geometries, Figures 21 and 22 , listed in the section 4. Each domain was simulated with the Reynolds number equal to 1, 10 and 30, with each one assuming the value of parameter $\sigma$ equal to 5, 10 and 30. The motivation to choose these Reynolds number was twofold. First, these were the same Reynolds numbers used in Andreasen (2017). Second, these low Reynolds numbers ensure that the simulations were in the laminar regime (below Reynolds 2000 based on duct flows (ÇENGEL; CIMBALA, 2013)) even if the optimized domain has a nozzle structure. Furthermore, this procedure was repeated for each objective function proposed in section 3 . Therefore, for each objective function, 27 simulations were carried out, and, in total, 81 models were simulated.

The first question answered was " is there any difference in the results generated by each objective functions?". To this end, the geometries obtained after the optimization process were filtered, leaving only the external contours. For a given Reynolds number and $\sigma$ value, the results were plotted of all three objective functions together. For sake of clarity, Figures 44 to 61 were positioned in the appendix A.

These pictures indicate that, in general, no significant differences were produced by the 
objective functions. Exceptions were noted when the Reynolds was set equal to 1 . At this value, the objective functions linked with the fluxes, at the secondary inlet $\left(J_{2}\right)$ and outlet regions $\left(J_{3}\right)$, produced the same geometry, whereas the objective function linked with the pressure $\left(J_{1}\right)$ produced geometries slightly different.

In such a case, the velocity magnitude at the primary inlet was smaller than the other simulations, i.e. Reynolds number equal to 10 and 30. Moreover, it was possible to observe that, as the loss inside the optimization domain was further allowed (parameter $\sigma$ was increased), the jet structure obtained after the optimization process increasingly elevated the quantity of internal momentum defined by equation 6.2 (Figures 29 for geometry 2 and 30 for geometry 3 ) and the differences in the final geometry reduced (as shown in Figures 44 to 46).

$$
\rho \int_{\Omega} \mathrm{U}^{2} d \Omega[\mathrm{J}]
$$

Figure 29 - Plot of the momentum integral over the optimization domain and geometric parameter $r_{p} / r_{t h}$ as function of parameter $\sigma$ with Reynolds equal to 1 .

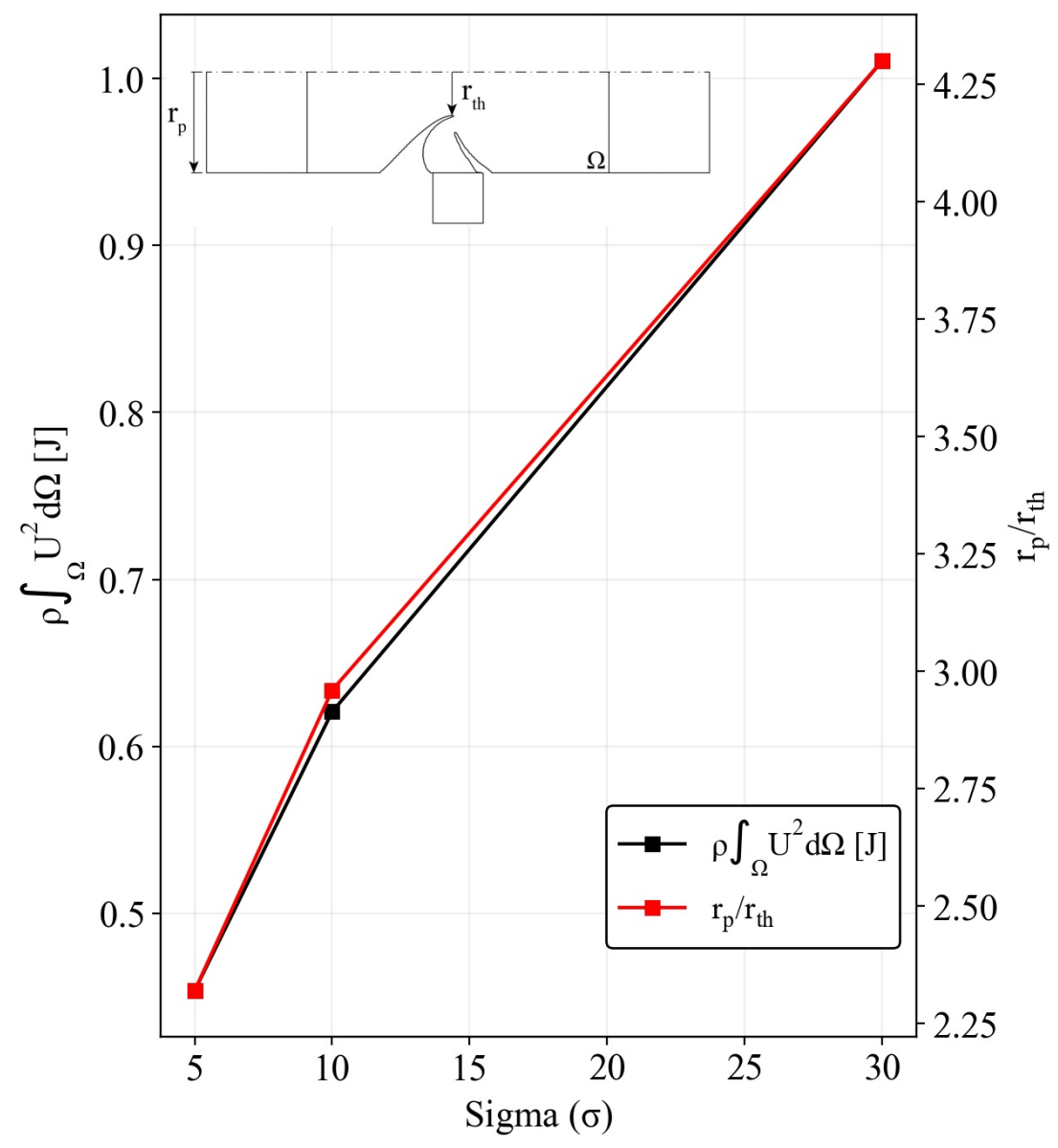

Source: Author. 
Figure 30 - Plot of the momentum integral over the optimization domain and geometric parameter $r_{p} / r_{t h}$ as function of parameter $\sigma$ with Reynolds equal to 1.

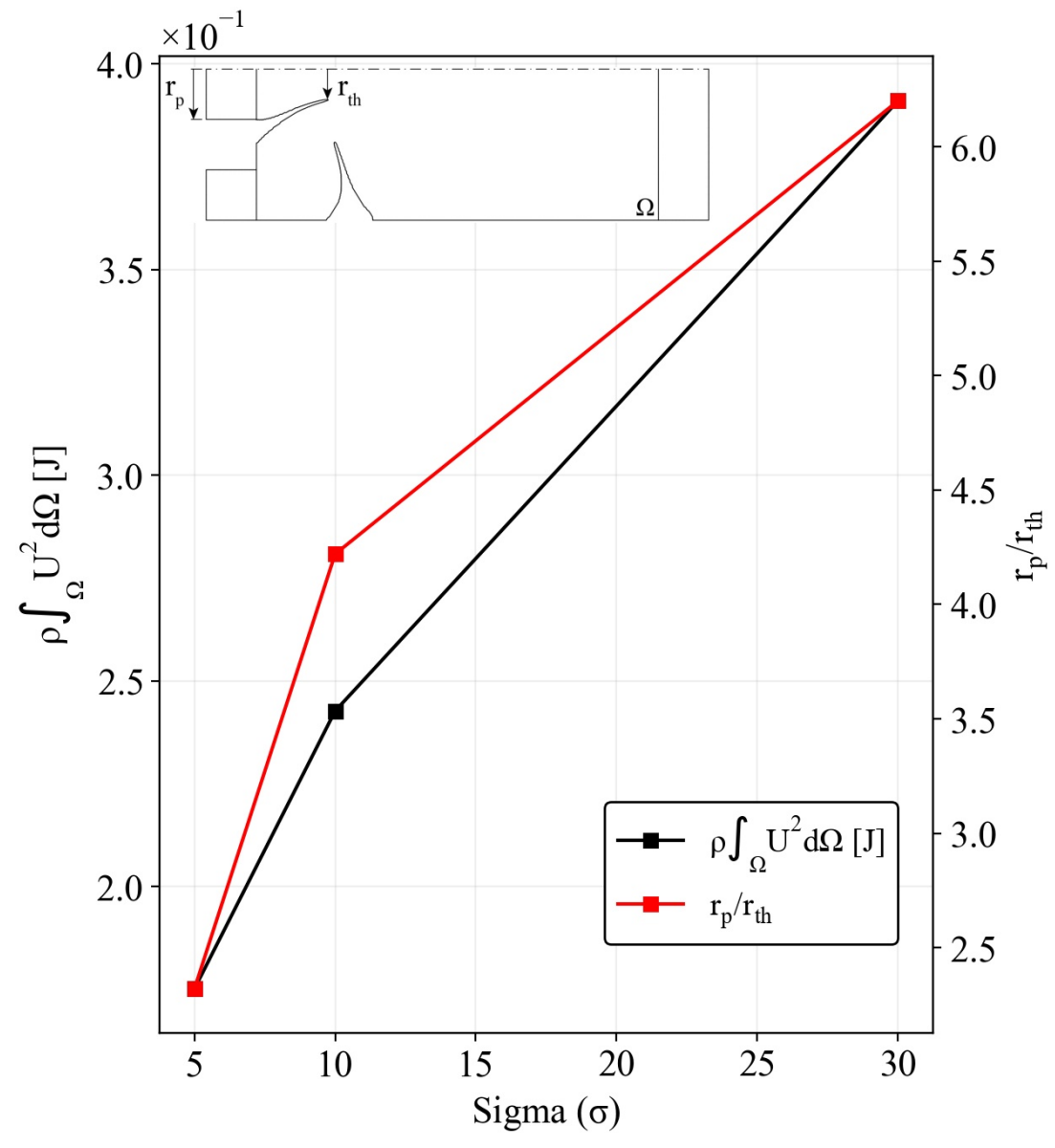

Source: Author.

Where $\mathrm{U}$ is the velocity magnitude field inside the hatched region (or $\Omega$ region). Moreover, it was plotted the flow ratio $M$ and the pressure coefficient (or $C p$, given by the equation 6.3) as a function of $\sigma$ for all Reynolds number and objective function (Figure 31 to the RSI and Figure 32 to the ASI) to complete the analysis with a quantitative comparison.

$$
C p=\frac{2\left(p_{\min }-\bar{p}_{\Gamma_{S}}\right)}{\rho \bar{U}^{2}}
$$

Where $p_{\min }$ is the minimum static pressure inside the optimized domain, $\bar{U}$ is the mean velocity calculated from the Reynolds number and the $\bar{p}_{\Gamma_{S}}$ is the average static pressure at the secondary inlet region.

From Figures 31 and 32, it is possible to conclude that the differences noted in the geometry comparison were most present in the pressure-related measures at low Reynolds numbers, whereas to the flow rate, the graphics did not show differences. To further verify the differences 
between the measures and better visualize the data due to the overlapping values, tables 3 to 6 were built to show the relative difference (given by equation 6.4) between the quantities used in Figures 31 and 32 considering the data collected from the results obtained by the objective function $J_{3}$ as the reference. The first two tables were related to the RSI and the last two to the ASI. At the last row of each table, the average value (AV) is depicted.

$$
\operatorname{Diff}_{\left(C_{p}, M\right) \%}=\frac{\left|\left(C_{p}, M\right)_{J 1, J 2}-\left(C_{p}, M\right)_{J 3}\right|}{\left(C_{p}, M\right)_{J 3}} \cdot 100
$$

Figure 31 - Flow rate $M$ and average static pressure at secondary inlet as functions of the parameter $\sigma$ for axial symmetric domain with radial secondary inlet. The values of $M$ and $C_{p}$ for Re 10 and 30 are overlapping.

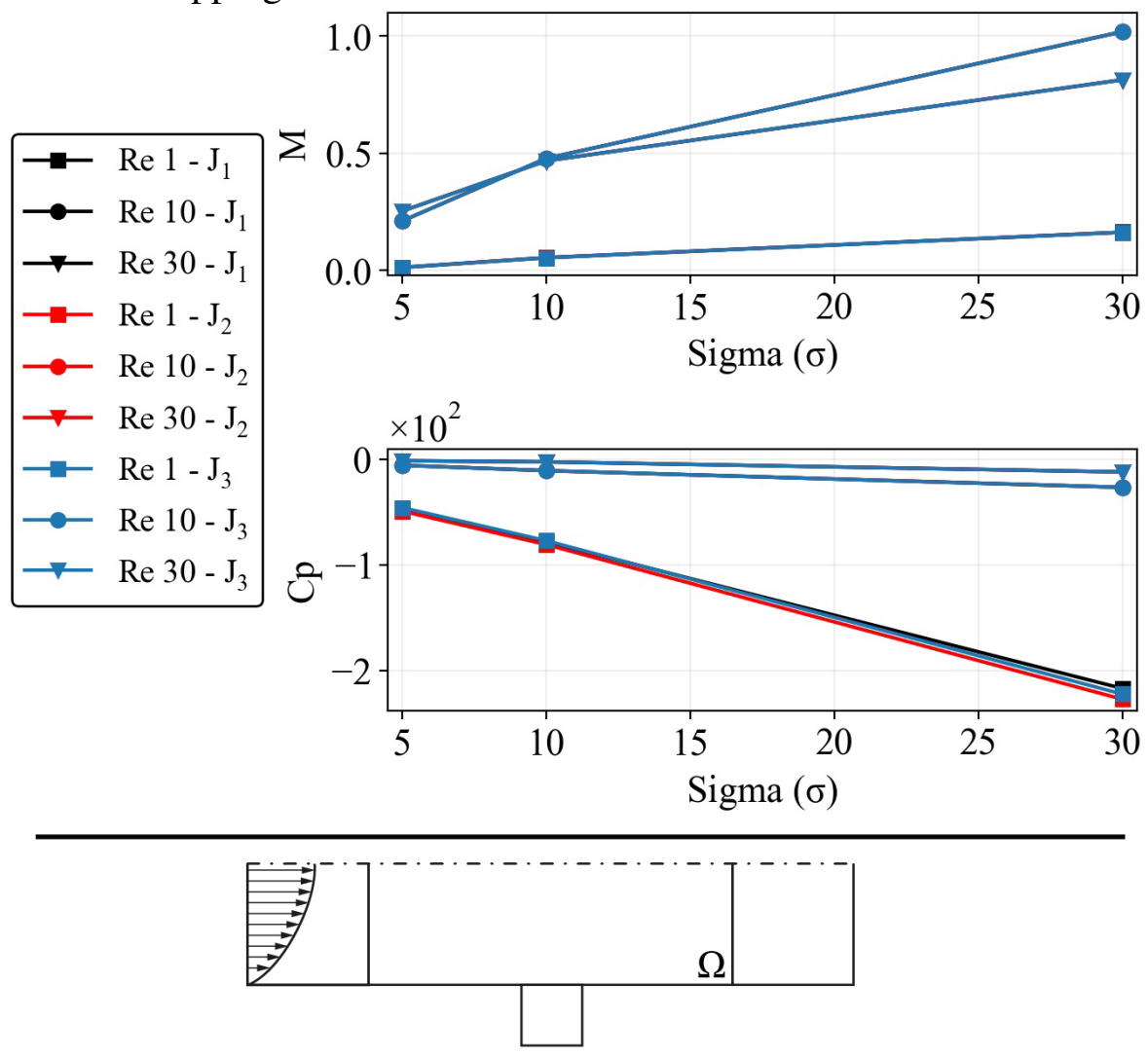

Source: Author. 
Table 3 - Relative difference between the values of $C p$ evaluated from the optimized domains obtained by each objective function to the radial secondary inlet. The values of domain calculated with $J_{3}$ was set up as reference All the used data is in percentage.

\begin{tabular}{|c|c|c|c|c|c|c|}
\hline \multirow{2}{*}{$\sigma$} & \multicolumn{2}{|c|}{ Reynolds $=1$} & \multicolumn{2}{|c|}{ Reynolds $=10$} & \multicolumn{2}{|c|}{ Reynolds $=30$} \\
\hline & $J_{1}-J_{3}$ & $J_{2}-J_{3}$ & $J_{1}-J_{3}$ & $J_{2}-J_{3}$ & $J_{1}-J_{3}$ & $J_{2}-J_{3}$ \\
\hline 5 & 1.76 & 7.22 & 0.34 & 0.19 & 0.02 & 0.03 \\
\hline 10 & 1.51 & 4.81 & 0.15 & 0.13 & 0.02 & 0.05 \\
\hline 30 & 2.40 & 2.18 & 0.02 & 0.00 & 0.06 & 1.48 \\
\hline $\mathrm{AV}$ & 1.89 & 4.74 & 0.17 & 0.11 & 0.03 & 0.52 \\
\hline
\end{tabular}

Source: Author.

Table 4 - Relative difference between the values of $M$ evaluated from the optimized domains obtained by each objective function to the radial secondary inlet. The values of domain calculated with $J_{3}$ was set up as reference All the used data is in percentage.

\begin{tabular}{|c|c|c|c|c|c|c|}
\hline \multirow{2}{*}{$\sigma$} & \multicolumn{2}{|c|}{ Reynolds = 1} & \multicolumn{2}{|c|}{ Reynolds $=10$} & \multicolumn{2}{|c|}{ Reynolds $=30$} \\
\hline & $J_{1}-J_{3}$ & $J_{2}-J_{3}$ & $J_{1}-J_{3}$ & $J_{2}-J_{3}$ & $J_{1}-J_{3}$ & $J_{2}-J_{3}$ \\
\hline 5 & 6.69 & 0.02 & 0.00 & 0.00 & 0.00 & 0.00 \\
\hline 10 & 1.35 & 0.52 & 0.00 & 0.00 & 0.00 & 0.00 \\
\hline 30 & 0.05 & 0.01 & 0.00 & 0.00 & 0.00 & 0.00 \\
\hline AV & 2.70 & 0.18 & 0.00 & 0.00 & 0.00 & 0.00 \\
\hline
\end{tabular}


Figure 32 - Flow rate $M$ and average static pressure at secondary inlet as a function of the parameter $\sigma$ for axial symmetric domain with axial secondary inlet. The values are overlapping.

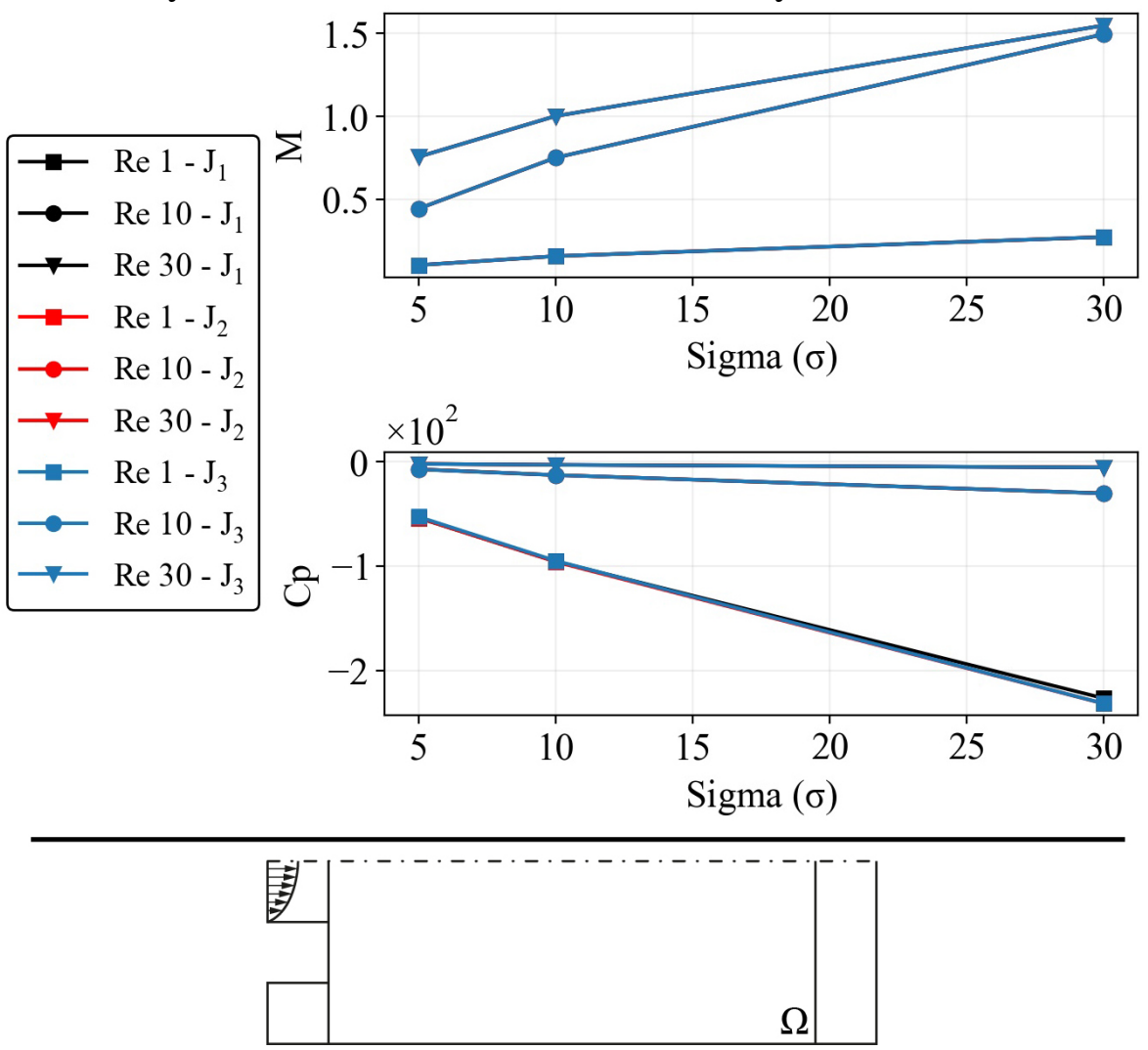

Source: Author.

Table 5 - Relative difference between the values of $C p$ evaluated from the optimized domains obtained by each objective function to the axial secondary inlet. The values of domain calculated with $J_{3}$ was set up as reference All the used data is in percentage.

\begin{tabular}{|c|c|c|c|c|c|c|}
\hline \multirow{2}{*}{$\sigma$} & \multicolumn{2}{|c|}{ Reynolds $=1$} & \multicolumn{2}{|c|}{ Reynolds $=10$} & \multicolumn{2}{|c|}{ Reynolds $=30$} \\
\hline & $J_{1}-J_{3}$ & $J_{2}-J_{3}$ & $J_{1}-J_{3}$ & $J_{2}-J_{3}$ & $J_{1}-J_{3}$ & $J_{2}-J_{3}$ \\
\hline 5 & 1.86 & 1.83 & 1.53 & 1.37 & 0.01 & 0.01 \\
\hline 10 & 0.61 & 1.01 & 0.11 & 0.12 & 0.97 & 0.00 \\
\hline 30 & 2.17 & 0.04 & 0.03 & 0.00 & 0.00 & 0.00 \\
\hline AV & 1.55 & 0.96 & 0.56 & 0.50 & 0.33 & 0.00 \\
\hline
\end{tabular}


Table 6 - Table of relative difference between the values of $M$ evaluated from the optimized domains obtained by each objective function to the axial secondary inlet. The values of the domain calculated with $J_{3}$ was set up as a reference. All the used data was in percentage.

\begin{tabular}{|c|c|c|c|c|c|c|}
\hline \multirow{2}{*}{$\sigma$} & \multicolumn{2}{|c|}{ Reynolds $=1$} & \multicolumn{2}{|c|}{ Reynolds $=10$} & \multicolumn{2}{|c|}{ Reynolds $=30$} \\
\hline & $J_{1}-J_{3}$ & $J_{2}-J_{3}$ & $J_{1}-J_{3}$ & $J_{2}-J_{3}$ & $J_{1}-J_{3}$ & $J_{2}-J_{3}$ \\
\hline 5 & 0.13 & 0.09 & 0.01 & 0.02 & 0.00 & 0.00 \\
\hline 10 & 0.05 & 0.00 & 0.00 & 0.00 & 0.01 & 0.00 \\
\hline 30 & 0.01 & 0.00 & 0.00 & 0.00 & 0.00 & 0.00 \\
\hline AV & 0.06 & 0.03 & 0.00 & 0.00 & 0.00 & 0.00 \\
\hline
\end{tabular}

Source: Author.

The data related to the fluxes objective functions $\left(J_{2}\right.$ and $\left.J_{3}\right)$ pointed almost no differences between the values of $M$ in all cases. This behavior was theoretical expected once the flow was incompressible and must satisfy the continuity equation (equations 3.6), nonetheless the verification was needed because the literature (ANDREASEN, 2017) pointed cases where objective functions $J_{2}$ and $J_{3}$ produced different results. For the measures concerned the difference between the objective function related to the pressure $\left(J_{1}\right)$ and flux objective function $\left(J_{3}\right)$ also to $M$ measure, the relative difference shown slightly higher values in the same cases where the geometrical differences were observed.

Major relative differences were observed for the measures of $C_{p}$, where its $\mathrm{AV}$ value tends to decrease (with exception of $J_{2}-J_{3}$ for RSI geometry) as the Reynolds number increase. The higher sensibility of the measures with regard to the pressure field was expected because the drawback of spurious pressure inside the solid model, this way, small differences in the final solid distribution can effect more the pressure field and consequently the $p_{\min }$ what translates in higher relative differences.

In the face of such dependence with the velocity, it was concluded that the objective functions produce slightly different results depending on the magnitude of inertia forces. However, considering the average value (AV) of the the relative difference of the tables 3 to 6 , there is 
no significant loss in considering the results of all objective function the same. Thus, to reduce the number of simulations, hereafter, all the analysis is made upon the data collected from the simulation that used the objective function $J_{2}$, once this objective function has a directly relation with the flow ratio $M$.

\subsection{Verification}

Since the density methodology uses a model for the solid region, it is essential to verify if the approach could correctly impose the solid condition to the flow. To this end, it was carried out simulations with the filtered domains depicted in Figures 44 to 61 with the same boundary conditions that were imposed on the optimization process, thus more 18 simulations were conducted. The objective function $J_{2}$ was plotted for all simulated Reynolds numbers as a function of the parameter $\sigma$ for the domain with radial secondary inlet and axial secondary inlet (Figure 33) to stress the difference between the topology optimization density approach and the verified geometry.

Figure 33 - Comparison between the $J_{2}$ value of the filtered and the optimized domain that used the density approach to the radial and axial secondary inlet.

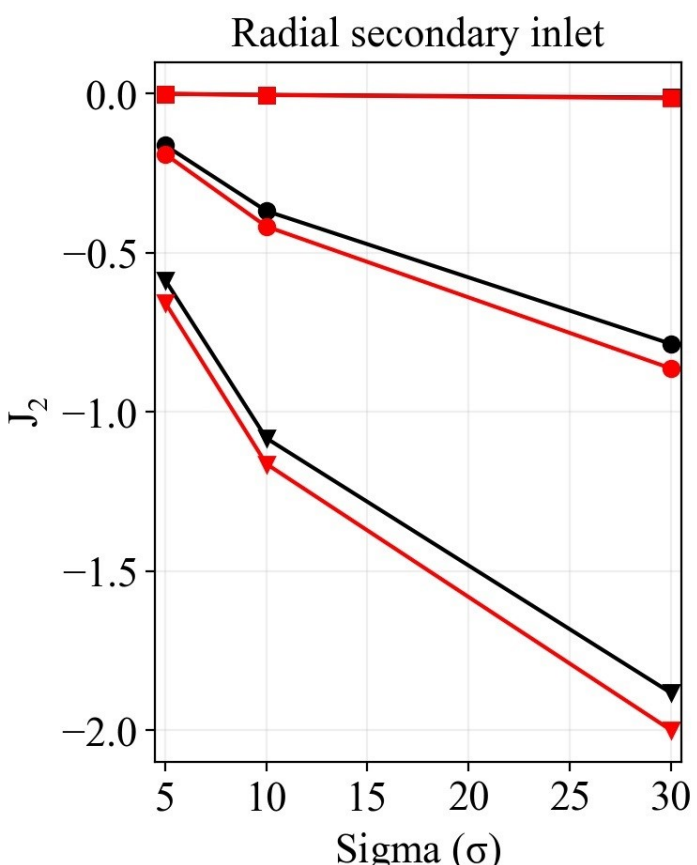

Sigma $(\sigma)$

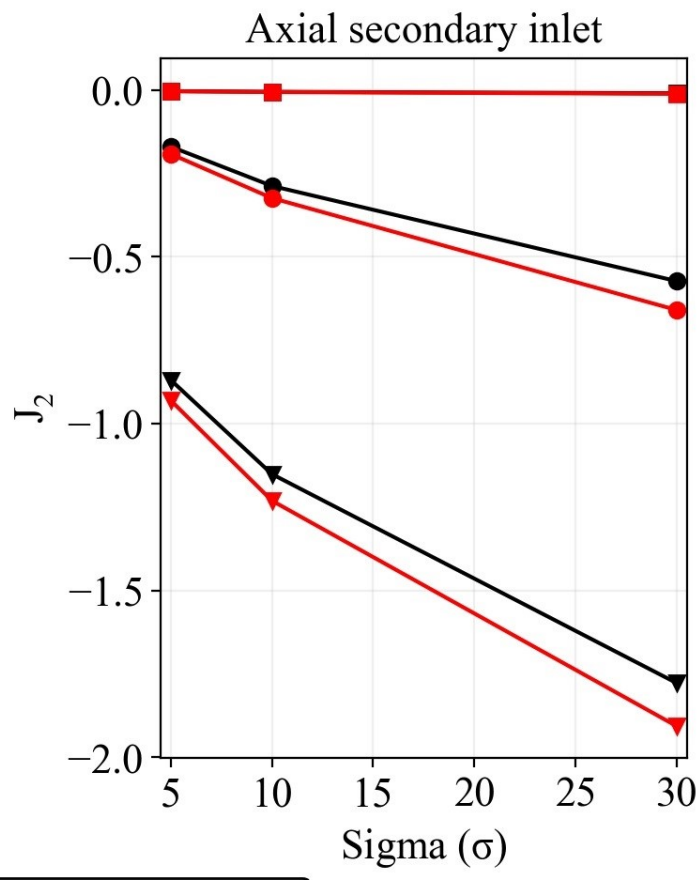

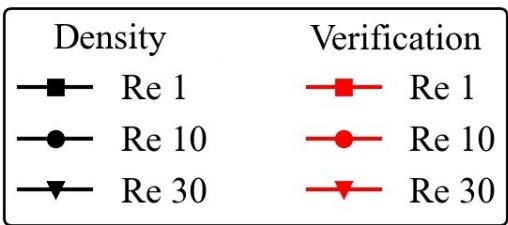

Source: Author. 
As depicted in Figure 33, the results obtained by the density approach were close to those obtained by the verification step for the Reynolds number equal to 10 and 30 . For the case with the Reynolds number equal to 1 , the graphic scale suppresses the deviations. To better visualize the data, table 7 presents the relative error between the verification and optimization simulations, where the former was considered the "exact" measure.

Table 7 - Table of relative error between the quantities measured in the verification and density optimization simulations of $J_{2}$ objective function for radial secondary inlet and axial secondary inlet geometries. All the used data is in percentage.

\begin{tabular}{|c|c|c|c|c|c|c|}
\hline \multirow{2}{*}{$\sigma$} & \multicolumn{2}{|c|}{ Reynolds = 1} & \multicolumn{2}{|c|}{ Reynolds $=10$} & \multicolumn{2}{|c|}{ Reynolds $=30$} \\
\hline & RSI & ASI & RSI & ASI & RSI & ASI \\
\hline 5 & 16.11 & 7.56 & 14.49 & 11.44 & 10.80 & 6.59 \\
\hline 10 & 13.83 & 9.62 & 11.65 & 11.20 & 6.94 & 6.46 \\
\hline 30 & 15.75 & 14.1 & 8.92 & 13.12 & 5.85 & 6.77 \\
\hline AV & 15.23 & 10.43 & 11.69 & 11.92 & 7.86 & 6.61 \\
\hline
\end{tabular}

Source: Author.

The data of table 7 pointed that the average error values tended to decrease with the Reynolds number increment and, in almost all cases, the error in each case was smaller for the ASI than to the RSI geometry. Furthermore, both geometries the error had its average value (AV) ranging from $15.23 \%$ to $6.61 \%$. Because the density methodology approach aims to model the solid region (not properly insert it in the domain) and the AV at high Reynolds number, target of the jet pump technology, were near the values present in the literature (about 7\%) (HAERTEL et al., 2018), the errors were considered acceptable. It is important to point out that the increase of the parameter $\alpha$ can represent a reduction in the errors. However, when used with higher values, the simulations presented problems of convergence. This error between a real solid interface and the solid model is inherent to the density approach selected to the study.

As the scope of this work is devices that have its secondary inlet in axial direction and no difficult were noted from geometry used by (ANDREASEN, 2017) to RSI geometry, the subsequent analysis will only focus on the ASI geometry. 


\subsection{Flow analysis}

As shown in the previous section, the characteristic of the flow ratio was benefited by the topology optimization and increase of parameter $\sigma$ (Figures 32 and 33). Nonetheless, there are other properties in the jet pumps that must be satisfied, so the final device works properly. In this case, the pressure efficiency (the second term in equation 1.7) needs to be, at least, kept constant during the constraint relaxation to ensure that the efficiency grows. If not, there is the possibility that the efficiency decreases despite the increase of $M$. It was plotted in Figure 34 the ejector's efficiency $(\eta)$ and the pressure efficiency $(N)$ for each Reynolds number as a function of $\sigma$ to verify this behaviour.

Figure 34 - The efficiency $(\eta)$ and pressure efficiency $(N)$ as a function of parameter $\sigma$ for each Reynolds number simulated.
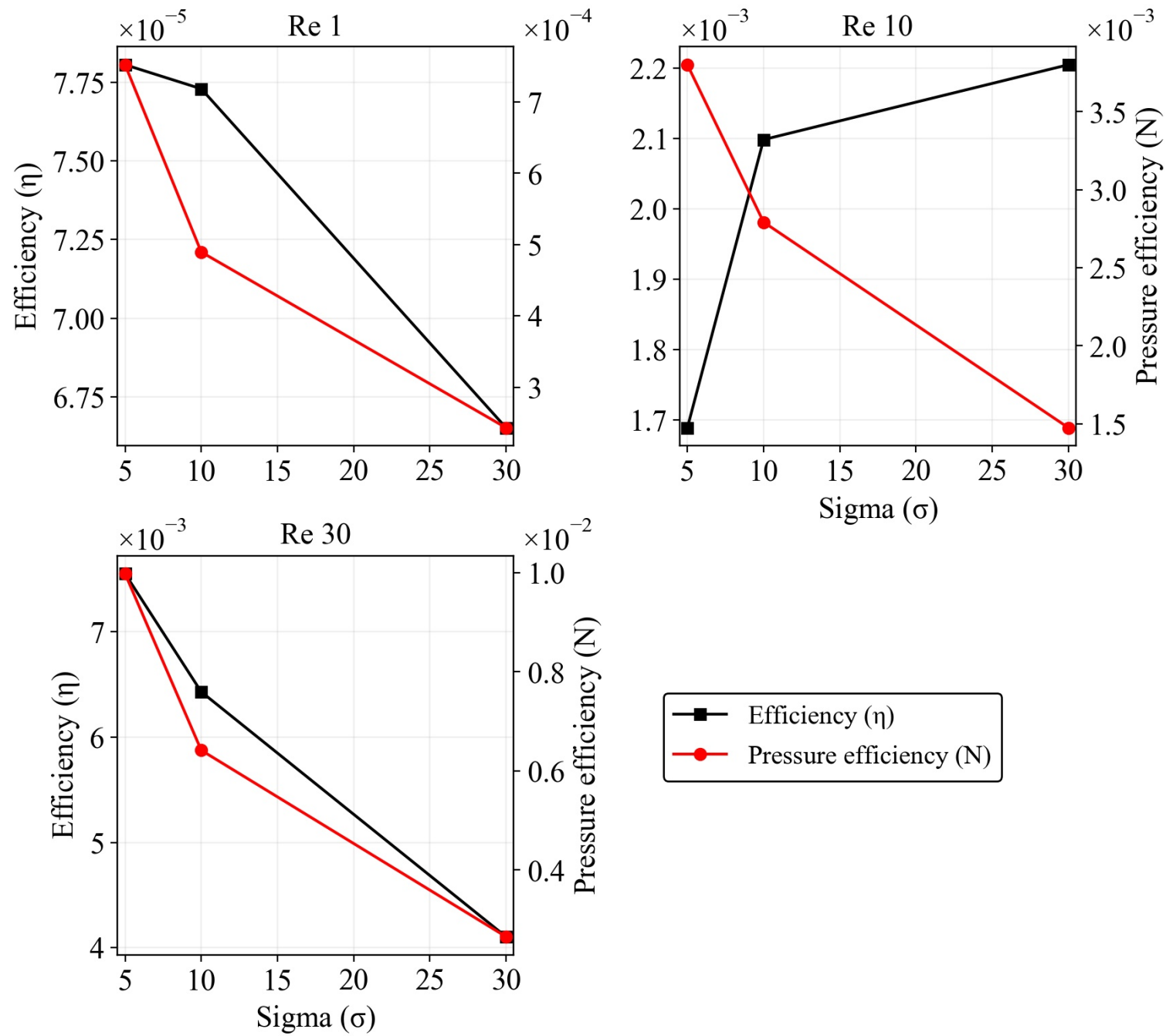

Source: Author.

As shown in this figure, the increment in the Reynolds number increased the efficiency 
$(\eta)$ values. Nonetheless, as discussed in the last paragraph, despite the gain in the flow ratio observed in Figure 32, the pressure efficiency decreased as the constraint was relaxed (parameter $\sigma$ increases). Especially in Reynolds number equal to 10, the gains of $M$ outweigh the loss in $N$. The reason for that was attributed to the slight relative difference between the values of pressure efficiency with $\sigma$ increment when compared to the other simulations with different Reynolds number (as pointed by table 8). Such combinations made it possible to achieve better values of efficiency the higher $\sigma$. Recording that,

$$
\eta=\overbrace{\frac{\dot{m}_{\text {secondary }}}{\dot{m}_{\text {primary }}}}^{M} \cdot \overbrace{\frac{\left(P_{\text {outlet }}-P_{\text {secondary }}\right)}{\left(P_{\text {primary }}-P_{\text {outlet }}\right)}}^{N}
$$

To the other Reynolds, the flow ratio does not outweigh the loss in the pressure efficiency. Thus, the efficiency $(\eta)$ curve decreased as the constraints were relaxed. Such behaviour stresses a trade-off between the gains in the flow ratio and pressure efficiency that, when the last was small enough, the efficiency can be improved. To further study the fluid dynamics that produced these phenomena, it was plotted the pressure contours, velocity field and streamlines seeded from the secondary and primary inlet (Figures 62 to 70). For clarity sake, the plots were positioned in appendix B.

Inspecting these plots, it was observed that, for all Reynolds number and $\sigma$ parameter, the structures of a nozzle and suction chamber were present. The former was responsible for accelerating the flow forming a jet that reduced the pressure inside the ejector domain (Venturi effect), whereas the last directed the flow to the low-pressure zone. In order to facilitate the subsequent discussions, it was plotted for each Reynolds the structure obtained from the parameter $\sigma$ equal to 5 (the value which all solid distribution resembles a jet pump despite the Reynolds number) with the dimensions that resemble a typical jet pump (Figure 35 to Reynolds equal to 1, Figure 36 to Reynolds equal to 10 and Figure 37 to Reynolds equal to 30). For the geometry of Reynolds number equal to 30, two red lines were drawn to subsequent analysis.

For the Reynolds number equal to 1, as the losses were further allowed, the suction chamber shifted in the direction of the outlet. The $N X P$ dimension gradually enlarge, whereas the radius of the outlet suction chamber $\left(r_{T h}\right)$ and the tip of the nozzle radius $\left(r_{N O u t}\right)$ gradually reduced. Meantime, after the secondary inlet structure, the flow had its direction abruptly changed. However, as a function of the low Reynolds number, no detachment was observed. Inspecting the behaviour of the corresponding efficiency curve in Figure 34, such configuration did not benefit 
the efficiency.

Figure 35 - Optimized geometry obtained for Reynolds equal to 1 and $\sigma$ equal to 5 plotted with its main dimensions.

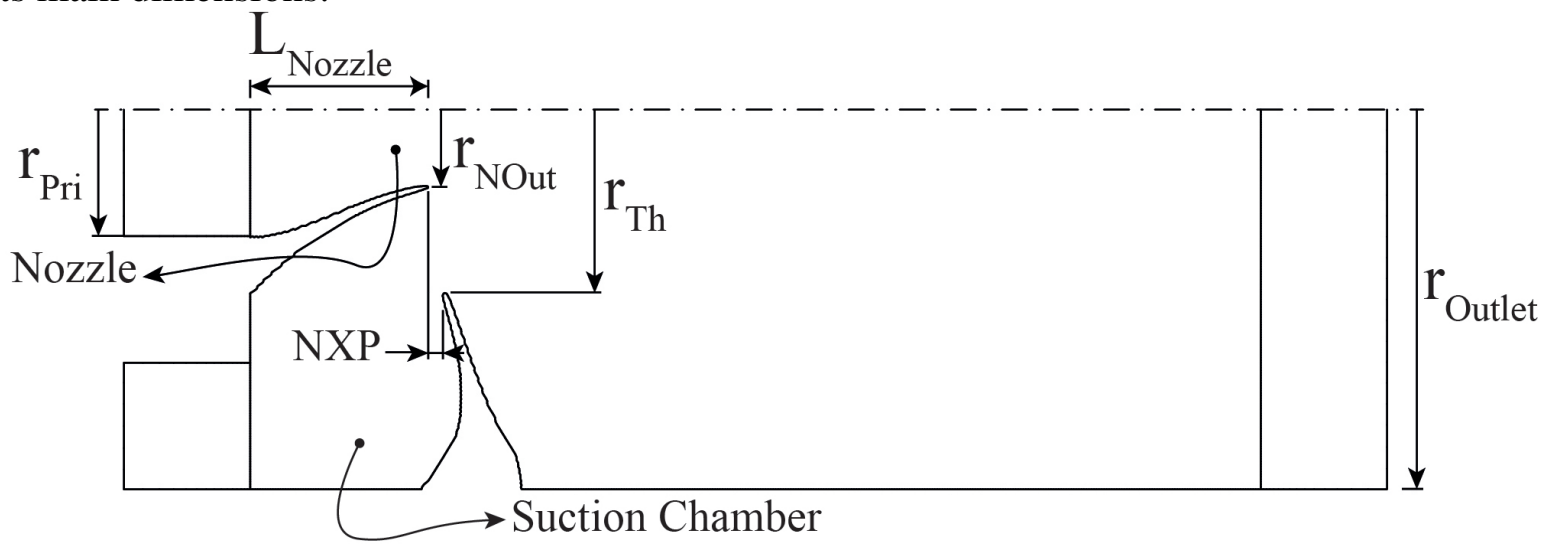

Source: Author.

Figure 36 - Optimized geometry obtained for Reynolds equal to 10 and $\sigma$ equal to 5 plotted with its main dimensions.

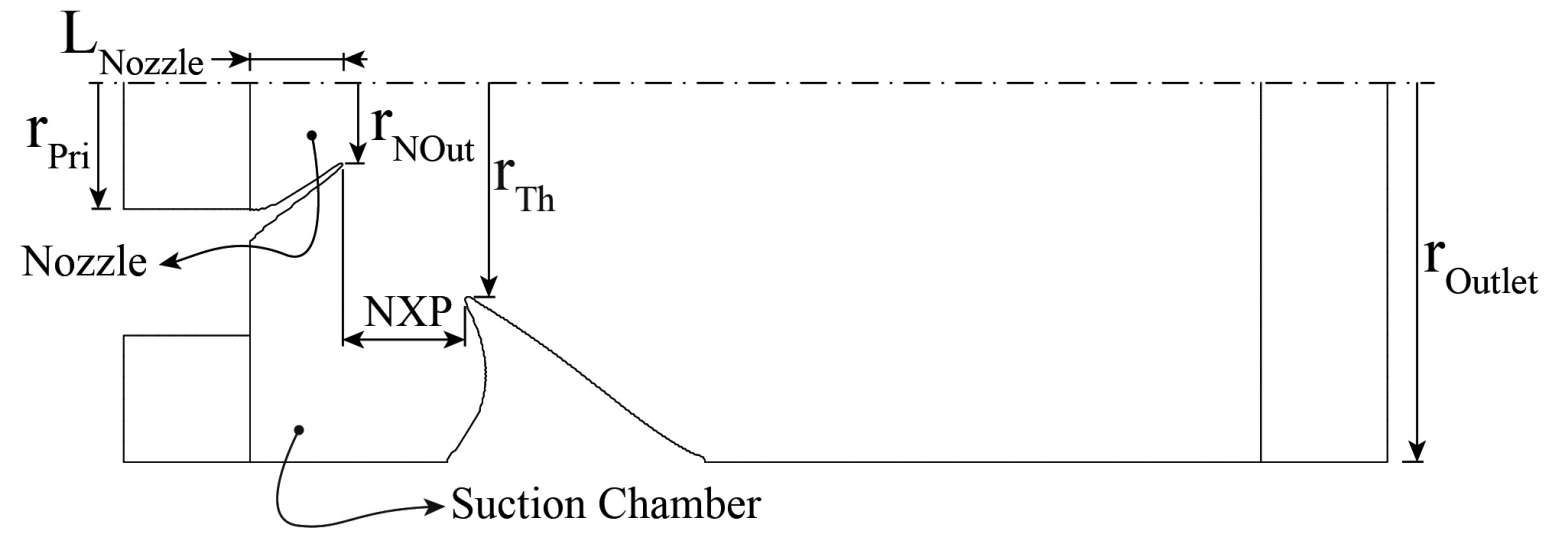

Source: Author. 
Figure 37 - Optimized geometry obtained for Reynolds equal to 30 and $\sigma$ equal to 5 plotted with its main dimensions and, in red, reference lines.

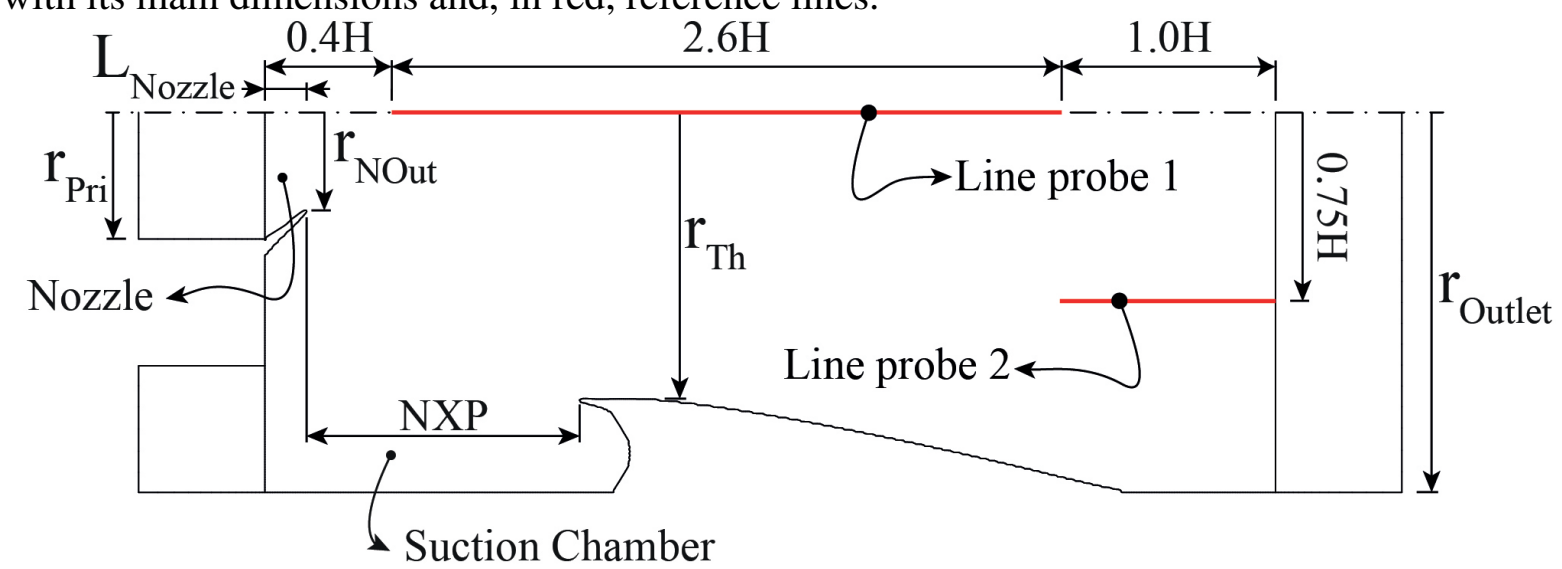

Source: Author.

For the Reynolds number equal to 10 , it was identified the same movement to the nozzle radius, and, with respect to its length, it was noted a reduction. The suction chamber radius was increased as well as the spacing between the outlet of the nozzle and upper part of the suction chamber (NXP dimension), which moved toward the outlet as the $\sigma$ increased. With Reynolds equal to 10 , the magnitude of the pressure efficiency lost between two consecutive values of $\sigma$ (table 8) was smaller among the simulations. Such behaviour was attributed to the structure formed after the suction chamber tip that, having a diffuser shape, promoted static pressure recovery. Hence, despite the trade-off between the flow ratio and pressure efficiency, the loss in the last due to the $\sigma$ increment was reduced, allowing the efficiency enhancement for higher $\mathbf{M}$.

Table 8 - Table of relative difference between two consecutive total pressure efficiency $N$ values. All used data are in percentage.

\begin{tabular}{ccc}
\hline \multirow{2}{*}{ Reynolds } & \multicolumn{2}{c}{$\sigma$} \\
\cline { 2 - 3 } & $5-10$ & $10-30$ \\
\hline 1 & -34.90 & -50.28 \\
\hline 10 & -26.54 & -47.20 \\
\hline 30 & -35.72 & -58.66 \\
\hline
\end{tabular}

Source: Author. 
For Reynolds number equal to 30, the same behaviour described above was observed for the nozzle radius, $N X P$, throat radius and nozzle length dimensions. However, exceptionally when the parameter $\sigma$ was incremented from 10 to 30, the nozzle length slightly increased and the throat radius retracted. In all geometries, the reduction of $r_{N O u t}$ was identified as the primary mechanism that linked the increment of $\sigma$ with large values of $M$ once this structure intensifies the jet flow favouring the Venturi effect. Nonetheless, for the geometries with Reynolds equal to 30, another structure near the outlet region appeared from $\sigma$ equal to 10 . This structure reduced the dimension fluid outlet area, further accelerating the flow, which reduced the static pressure (evidenced by Figure 38) inside the domain and enhanced the drag of fluid at the secondary inlet. Although this mechanism had benefited the $M$, the pressure efficiency $(N)$ was severely affected by the $\sigma$ growth, which proportioned the reduction of the efficiency (as depicted in Figure 34). Comments were made concerned the recirculation zones near the boundary condition in appendix E. Table 9 summarizes the trends in the geometries discussed above.

Figure 38 - Plot of the normalized static pressure $\left(p_{i} *\right)$ measured along the non dimensional probe line 1 and $2\left(z_{i} *\right)$ for Reynolds number equal to 30 and $\sigma$ equal to 5,10 and 30 . The reference static pressure to $p_{1} *$ normalization was $26.45 \mathrm{~Pa}$ and to $p_{2} *$ was $11.63 \mathrm{~Pa}$.
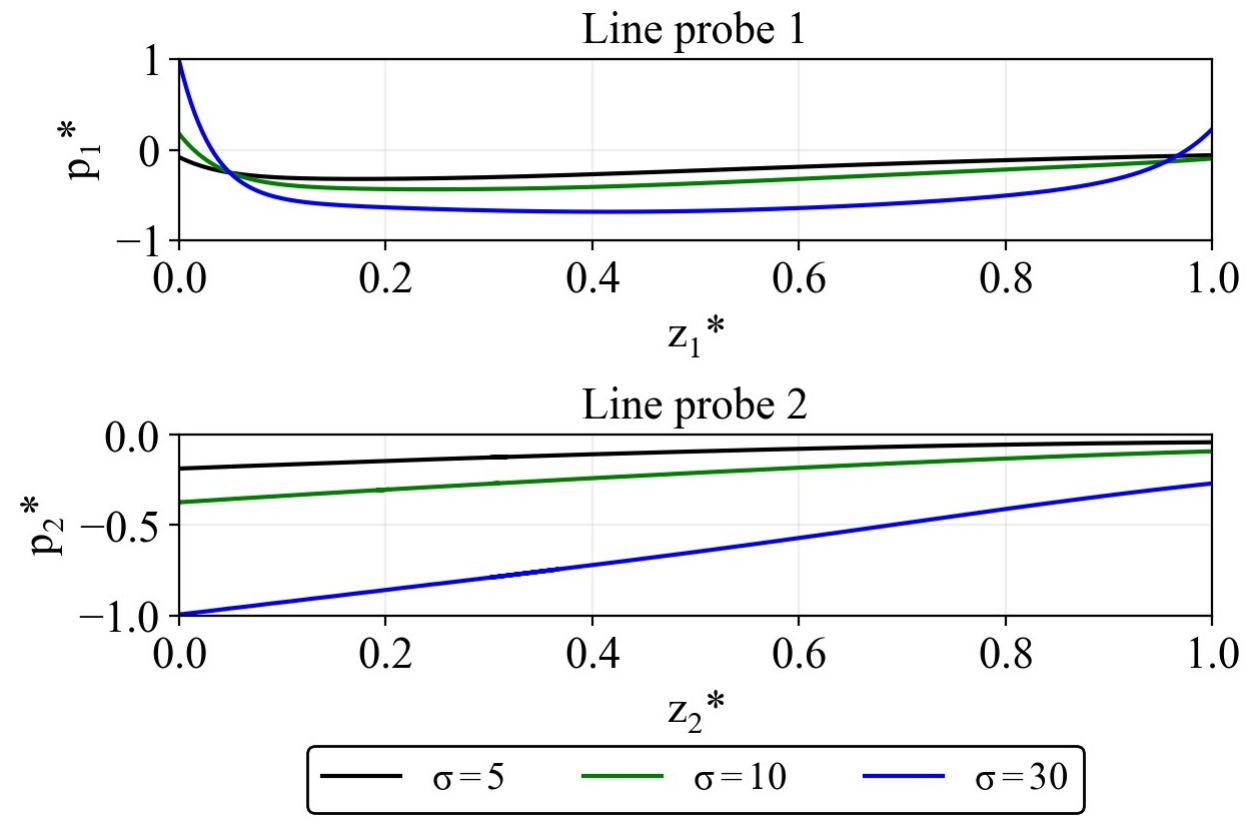

Source: Author.

It is evident that the solution present so far can not fully capture the physics of the ejectors, generating optimized geometries that sometimes benefit the efficiency (the case of Reynolds number equal to 10) and sometimes not (the cases of Reynolds number equal to 1 and 30). This happens because the objective function $J_{2}$ just benefited the flow ratio characteristic by the 
Table 9 - Geometrical trend during the parameter exploration.

\begin{tabular}{|c|c|c|c|c|c|c|c|c|c|}
\hline \multirow[b]{2}{*}{$\sigma$} & \multicolumn{3}{|c|}{ Reynolds = 1} & \multicolumn{3}{|c|}{ Reynolds $=10$} & \multicolumn{3}{|c|}{ Reynolds $=30$} \\
\hline & 5 & 10 & 30 & 5 & 10 & 30 & 5 & 10 & 30 \\
\hline$b$ & 0.19 & 0.11 & 0.06 & 0.14 & 0.09 & 0.05 & 0.12 & 0.09 & 0.06 \\
\hline$S$ & 0.19 & 0.33 & 0.68 & 1.51 & 2.34 & 4.54 & 2.82 & 3.69 & 6.21 \\
\hline$c$ & 4.53 & 4.61 & 5.24 & 3.12 & 3.12 & 2.89 & 1.76 & 1.74 & 1.80 \\
\hline$L_{T h} / d_{N O u t}$ & 2.31 & 3.28 & 5.61 & 1.16 & 1.51 & 2.16 & 0.43 & 0.58 & 0.84 \\
\hline
\end{tabular}

Source: Author.

augmentation of the flow at the secondary inlet and the constraint just deals with the loss inside the domain. The $N$ term was not considered in the analysis, and, therefore, the $\eta$ obtained in each sigma/Reynolds was not optimized.

$$
\eta=\overbrace{\frac{\overbrace{\text { secondary }}^{\dot{m}_{\text {primary }}}}{J_{2}}}^{J_{\text {Imposed }}} \cdot \overbrace{\frac{\left(P_{\text {outlet }}-P_{\text {secondary }}\right)}{\left(P_{\text {primary }}-P_{\text {outlet }}\right)}}^{N}
$$

There were two paths to correct it. One would be the introduction of another constraint in the flow ratio and the objective function aiming to minimize the difference between the static pressure at the primary inlet and outlet or, in a relative point of view, a constraint in the static pressure drop between the primary inlet and outlet with the objective to maximize the flow ratio. On the other hand, it could be introduced a second objective function and transform the original problem into a multi-objective one.

Because of the nature of the relation between the $M$ and $N$, which was shown inversely proportional, was drawn a comparison with the jet pump problem and the heat sink problem, where it is aimed the maximum heat transfer with the minimum pressure drop. In the literature, this optimization problem is often treated using the multi-objective approach (KOGA et al., 2013; DEDE, 2009; TAWK; GHANNAM; NEMER, 2019). For this reason, the same approach was selected for the present study. 


\subsection{Multi-objective approach}

After several tests (even with the total pressure efficiency $N$ ), the additional objective function that was shown to complete the physics of jet pumps is given by the equation

$$
J_{4}=\left(\frac{1}{L_{\Gamma_{P}}} \oint_{\Gamma_{P}} p d \Gamma_{P}-\frac{1}{L_{\Gamma_{O}}} \oint_{\Gamma_{O}} p d \Gamma_{O}\right)^{2}
$$

Where the first term of equation 6.7 is the average static pressure at the primary inlet, the second term of equation 6.7 is the average static pressure at the outlet, $L_{\Gamma_{P}}$ is the length of the primary inlet boundary condition and $L_{\Gamma_{O}}$ is the length of the outlet boundary condition. The difference was squared, so the minimum possible value of the objective function was equal to zero and not a negative value. It was selected the sum of objectives that gives rise to other two problems to treat the multi-objective problem: sum of two distinct quantities (pressure and flow rate) and two scales that were not close. To tackle it, the methodology used by Tawk, Ghannam \& Nemer (2019) results in

$$
J_{\text {Sum }}=w \cdot \frac{J_{4}-\overline{J_{4}}}{\underline{J_{4}}-\overline{J_{4}}}+(1-w) \cdot \frac{J_{2}-\overline{J_{2}}}{\underline{J_{2}}-\overline{J_{2}}}
$$

Where $w$ weights in how much of each characteristic represented by the objective functions $\left(J_{2}\right.$ and $J_{4}$ ) was taken into account during the $J_{S u m}$ evaluation, the overbar symbol represent the values of the objective function evaluated with $w$ equal to one and the underline represent the value of the objective functions evaluated with $w$ equal to zero. The other optimization variables like boundary conditions, constraints, mesh and optimization solver configuration were the same that used in the single objective function simulation. The methodology was applied in the case of Reynolds number equal to 30 and $\sigma$ equal to 30 .

\subsubsection{Multi-objective results}

The second round of results are presented in two forms. One depicting the jet pump metrics $M, N$ and $\eta$ as function of the $w$ multi-objective parameter (Figure 39) and another plotting the static pressure contours, solid distribution, stream lines (seeded from the primary and secondary inlet) and velocity colour map for each $w$ (Figures 71 to 86 in appendix C).

Figure 39 shows the relationship between the flow rate and total pressure efficiency described in the previous section. As the weight $(w)$ was increased, the static pressure difference 
between the secondary inlet and outlet was further taken into account, the $M$ dropped. In the Figures of appendix $\mathrm{C}$ (Figures 71 to 80 ), this relation was observed in the progressive reduction of the structure attached to the centerline until its disappearance, the nozzle diameter increment and the movement of secondary inlet structure towards the primary inlet. This trend augmented the jet pump efficiency up to the $w$ equal to 0.8 , establishing a plateau. For $w$ equal to 0.9 up to 0.975 , the nozzle disappears, and the efficiency $(\eta)$ decreased to a new plateau where no significant change in the flow field was observed (Figures 81 to 83 )

Between $w$ equal to 0.99 and 0.995 , the jet pump assumed another configuration, the flow ratio exercised even less influence and had its value diminished, the nozzle structure was substituted by a semi diffuser (that still allows the entrainment of fluid from the secondary inlet) and the secondary inlet structure was reduced. With higher values of $w$, the semi diffuser grew up to completely close the secondary inlet. 
Figure 39 - Plot of the metrics $M, N$ and $\eta$ as function of the weight $w$.

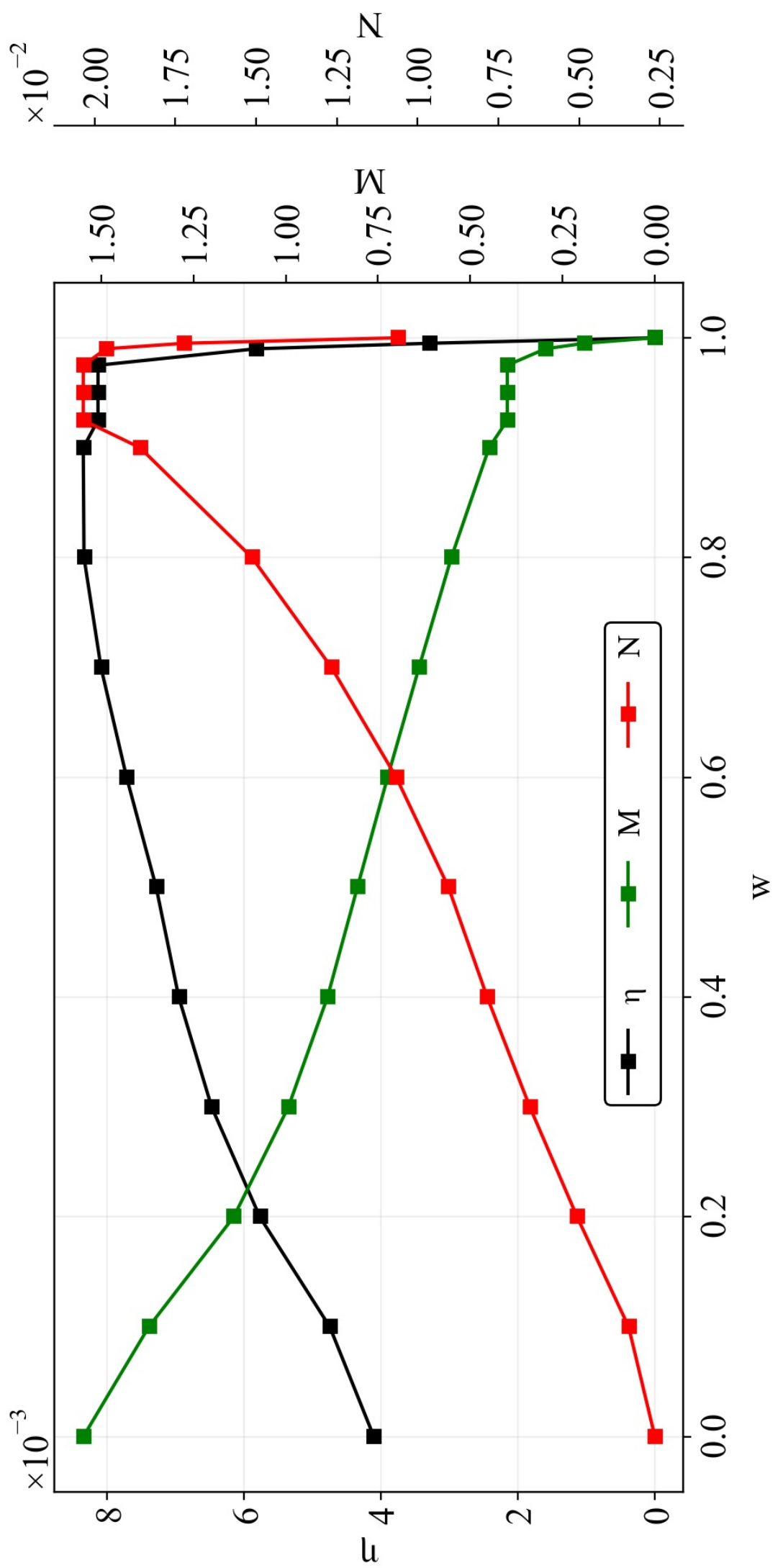

Source: Author. 
For $w$ equal to 1, the drastic reduction in the metrics evidenced that the optimized structure lost its operation principle, once for this case the multi-objective function considers only the static pressure difference $(w=1)$ and the flow through the secondary inlet had no weight $(w=0)$. Because of the blocked secondary inlet, the flow ratio went to zero converting the jet pump into a diffuser. As a consequence, the efficiency went to zero, and the $N$ decreased (once $P_{\text {secondary }}$ lost its term related to the velocity and $P_{\text {outlet }}$ had its velocity term decreased).

The best efficiency was achieved with the weight between 0.8 and 0.9. At these values, a gain of $102 \%$ compared to the final geometry obtained by the single objective function. Because of the available time, it was not possible to apply the multi-objective function with other Reynolds numbers and sigmas. Recording that:

$$
g(p) \equiv \frac{\oint_{\prod_{P}} p d \Gamma}{\sigma \oint_{\Gamma_{P}} p_{r e f} d \Gamma}-1
$$

\subsection{Alternative approach - single objective function}

As already discussed, there was the possibility to use a single objective function to enhance the total pressure efficiency with the introduction of another restriction to ensure a flow rate at the secondary inlet. To test this option, another simulation with the $J_{4}$ objective function and the flow rate at the secondary inlet restricted by $g_{s e c}$ (given by equation 6.12) was set up. Additionally, as the constraint of inlet pressure $(g(p))$ is not usual in the topology optimization literature, the division of the design variable integral over optimization region ( $\Omega$ region) divided by the volume of the same region (or solid volume constraint $g_{\text {new }}$ given by equation 6.11) was used in place of the pressure constraint. So, the new optimization problem can be defined as:

$$
\begin{array}{ll}
\text { Minimize : } & J_{4}(N(\breve{\xi}), \breve{\xi}) \\
\text { Subjected to: } & \text { 1) } \psi_{\text {inf }} \leq g_{\text {new }} \leq \psi_{\text {sup }} \\
& \text { 2) } g_{\text {sec }} \geq Q_{\text {ref }} \\
\text { 3) } 0 \leq \breve{\xi} \leq 1
\end{array}
$$

Where $\psi_{\text {sup }}$ is the upper limit of the volume constraint, $\psi_{\text {inf }}$ is the bottom limit of the volume constraint, $Q_{\text {ref }}$ is a predetermined flow rate at the secondary inlet and $\breve{\xi}$ is the design variable. 
Defining,

$$
g_{\text {new }} \equiv \frac{\int_{\Omega} \breve{\xi} d \Omega}{\operatorname{Vol}_{\Omega}}
$$

And,

$$
g_{s e c} \equiv-\int_{\Gamma_{S}} u_{n} d \Gamma \geq Q_{r e f}
$$

With $\operatorname{Vol}_{\Omega}$ being the total volume of optimization. Constraint number 1 is inherent to the topology optimization. Without it, the process is too loose, and convergence will not happen. Constraint number 2 was necessary to ensure that the simulation does not converge to a solution where the secondary inlet is closed. Constraint number 3 is natural from the density method.

The case chosen to run the multi-objective simulations was the one with Reynolds number and parameter $\sigma$ equal to 30 due to the presence of a structure that acts unfavourably under static pressure recovery in the first round of simulations. Thus, it is expected to clearly observe the influence of the new objective function under the optimized solid-void distribution. The other parameters of the optimization were $Q_{\text {ref }}$ equal to $0.7 \mathrm{~m}^{2} / \mathrm{s}, \psi_{\text {inf }}$ equal to 0.85 and $\psi_{\text {sup }}$ equal to 0.95 selected based on the results of the multi-objective function approach with pressure constraint.

\subsubsection{Results of the alternative approach (single objective function)}

Inspecting the external contours extracted from the optimized domains (Figure 40), it is possible to qualitatively infer that the same geometry was obtained by the single objective function (SOF) with volume constraint and multi-objective function (MOF) with pressure constraint. The case of the multi-objective function that matches with the single objective function was for the $w$ equal to 0.7 once the flow rate at the secondary inlet was 0.73 for the MOF and $0.70 \mathrm{~m}^{2} / \mathrm{s}$ for the SOF. With respect to the averaged volume factor (that in SOF is set up as the constraint $\left.g_{\text {new }}\right)$, the MOF had the value of 0.94 and SOF 0.89 . The similarity between the two approaches lies in the fact that the set up of the single objective function was based in the result of multi objective function (i.e. values of $\psi_{\text {sup }}, \psi_{\text {inf }}$ and $Q_{\text {ref }}$ ). 
Figure 40 - Geometric comparison between the two approaches.

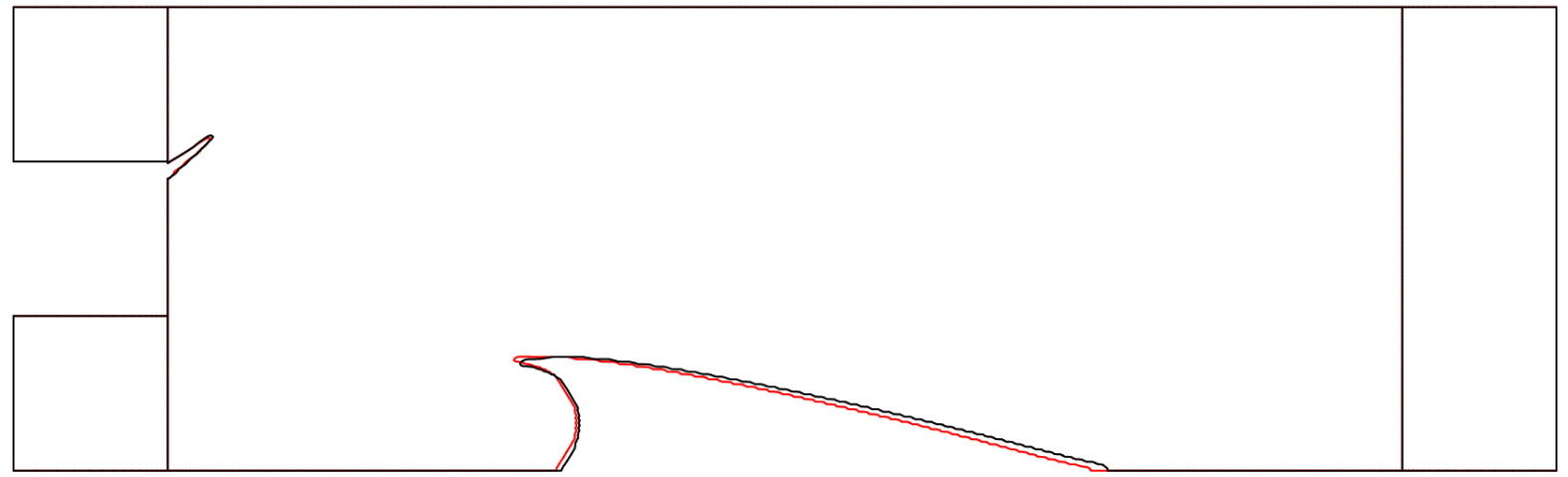

Multi-objective function (Pressure constraint)

Single objective function (Volume constraint)

Source: Author.

Additionally, the velocity (Figure 42) and pressure field (Figure 43) as well as the stream lines was plotted (Figure 41). The similarity in these figures reinforce the statement made above.

Figure 41 - Comparison between the stream lines (black seeded from the primary inlet, red from the secondary inlet and blue at the recirculation zone) obtained by the two approaches.

Volume constraint

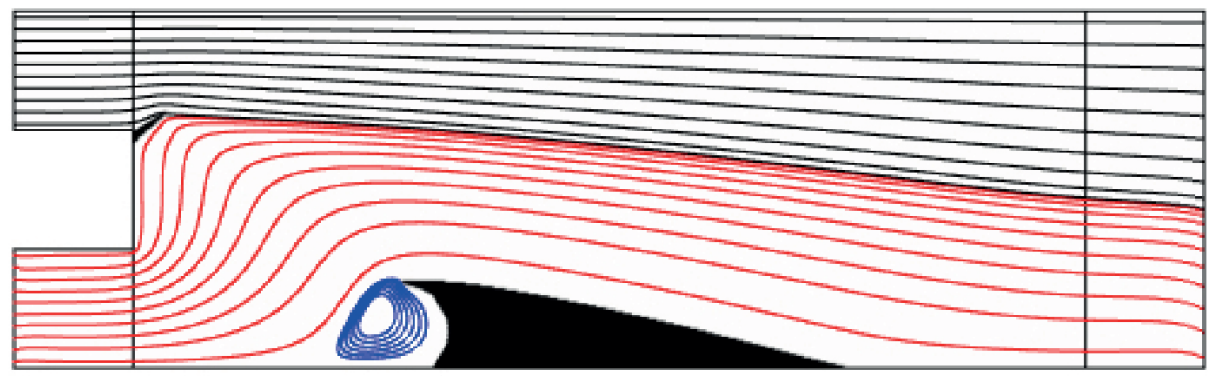

Pressure constraint

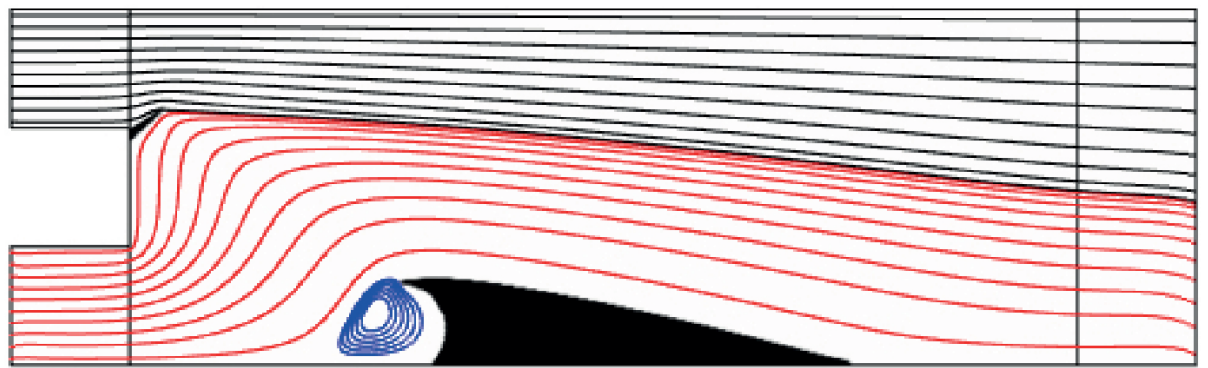

Source: Author. 
Figure 42 - Comparison between the velocity field obtained by the two approaches. At the upper part, the volume constraint and, at the bottom, the pressure constraint.

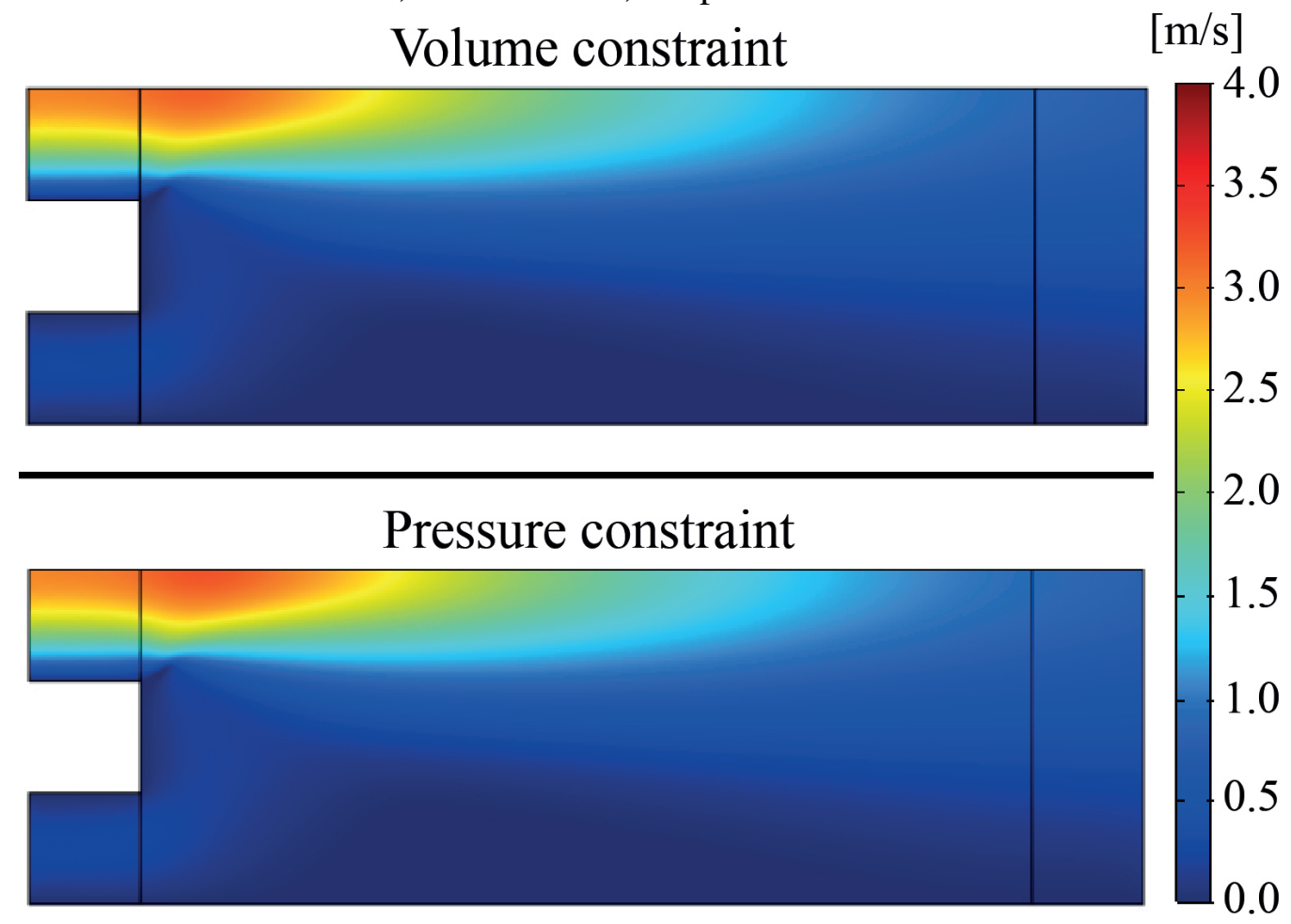

Source: Author. 
Figure 43 - Comparison between the pressure field obtained by the two approaches. At the upper part, the volume constraint and, at the bottom, the pressure constraint.

Volume constraint

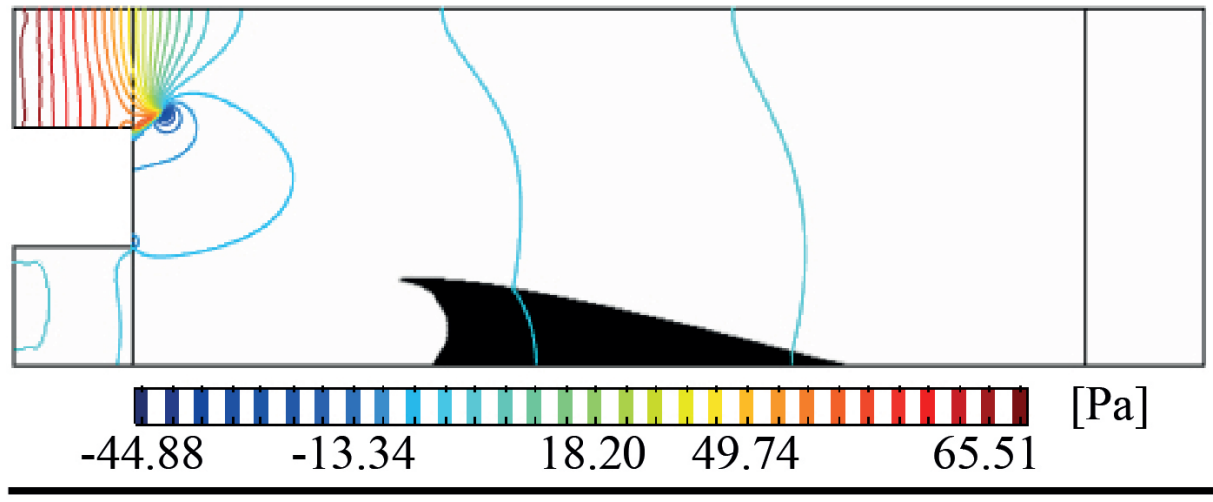

Pressure constraint

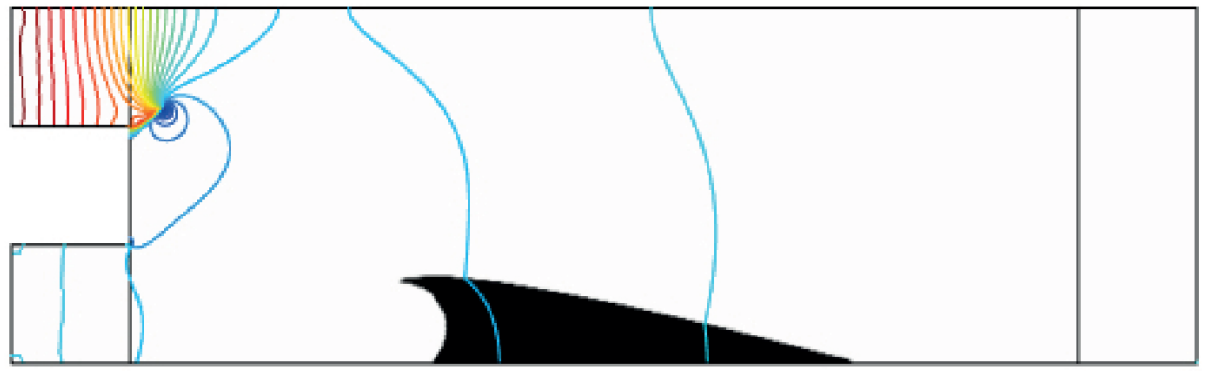

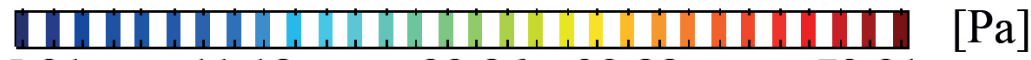
$\begin{array}{lllll}-45.21 & -11.18 & 22.86 & 39.88 & 73.91\end{array}$

Source: Author.

With respect to the efficiency, the volume constraint had a value equal to $0.82 \%$ and the pressure constraint equal to $0.81 \%$. In the face of this evidence, it was possible to conclude that both techniques could optimize and complete capture the ejector physics. 


\section{CONCLUSION}

The topology optimization method was applied to the field of jet pumps with the following simplifications of the flow field: incompressible, laminar, adiabatic, without body forces and in steady-state to make possible the application of the density approach in the available time. Indeed, the assumptions end up limiting the application range of ejectors. However, it still represents the main physics behind jet pumps. Thus, the results and discussions in the present study fill gaps in the field of the topology optimization applied in ejectors and the methodology can be used in future works that treat more complex flow.

In the section 5, the simulations indicated that if the number of iterations in each step increased, the objective function was benefited and the final solid distribution converged. This way, the number of iterations proposed to this study had its valued increased (600 in each step) with relation to the base article of Andreasen (2017). The change improved in $9.4 \%$ the the objective function compared with the case that uses the same number of iterations of the base paper.

It was verified that the first group of objective functions proposed by Andreasen (2017) $\left(J_{1}, J_{2}\right.$ and $\left.J_{3}\right)$ was able to enhance the flow rate characteristic of the ejectors and produce the same geometry. However, slight differences were noted for Reynolds number equal to 1 and parameter $\sigma$ equal to 5. These differences tend to disappear with higher parameter $\sigma$ and Reynolds number.

Although the simulations with Reynolds number equal to 10 present desired characteristics of a jet pump (i.e a nozzle and suction chamber), the simulations with Reynolds number equal to 1 and 30 pointed that the first set of objective functions could not capture the physics of static pressure recovery. Furthermore, the flow ratio $(M)$ and total pressure efficiency $(N)$ showed to be inversely proportional. As the $M$ increased, the $N$ decreased. Such characteristic complies with the working principle stressed in section 1 where the specific energy provided by the external source is dissipated higher the flow ratio $M$.

The multi-objective function approach was investigated between the two possibilities to treat this problem (due to its similarity with heat-sink problems). The tests showed that the squared difference between the static pressure at the primary inlet and the outlet $\left(J_{4}\right)$ and the 
measure of flow at secondary inlet $\left(J_{2}\right)$ were the most suitable functions to conduct topology optimization in jet pump field. The results show that the efficiency can be improved up to $102 \%$ by the method. Alternatively, the option of using a single objective function (squared difference between the static pressure at the primary inlet and the outlet) and another restriction (minimum flow rate at the secondary inlet) as well as the substitution of the pressure constraint by the volume constraint, was briefly tested and no significant differences were noted between both approaches. This result indicate that both constraints (volume and pressure) are equivalent to the case of jet pumps.

Future works can further investigate the optimal number of iterations of the methodology and increase the flow complexity by means of turbulence models. Still, more complex topology optimization techniques like level-set and topology optimization of binary structures (TOBSGT) can provide more insights into the field of topology optimization in jet pumps and overcome the difficult of the density approach to model the solid and the interaction of the fluid with it.

The discussion in the appendix E evidenced that the extension of the domain can affects the final solid and void distribution. Future works should investigate more about this subject.

Despite of the results presented in this dissertation shown unusual geometries of jet pumps, more optimization must be conducted to introduce other features like structural analysis, manufacturability and resonance frequency.

The axisymmetric flow was adopted to save computational time. However, as discussed in the literature review, there is the possibility of the optimized jet pump structure be nonaxisymmetric. This way, further investigation needs to clarify if the axisymmetric flow was imposed on the simulations or was the best solution for jet pumps. Also, the geometric and flow parameters can assume a number of configurations, which can substantially affect the behaviour of the optimized geometry. This way, further investigations can be made to point the best relation between these parameters.

Future works can improve the complexity of the topology optimization in jet pump field carrying out optimizations in turbulent and compressible fields. Such advance will march closer to the final objective of the RCGI project, i.e. biphasic, turbulent and compressible. 


\section{REFERENCES}

Jet Pump Performance With Secondary Fluids Differs in Density and Viscosity From Primary Fluid, All Days de Abu Dhabi International Petroleum Exhibition and Conference, (Abu Dhabi International Petroleum Exhibition and Conference, All Days).

ALLAIRE, G. et al. The homogenization method for topology optimization of structures: Old and new. Interdisciplinary Information Sciences, v. 25, n. 2, p. 75-146, 2019.

AMESTOY, P. R. et al. MUMPS: a general purpose distributed memory sparse solver. In: SPRINGER. International Workshop on Applied Parallel Computing. [S.1.], 2000. p. 121-130.

ANDERSON, J. Computational Fluid Dynamics: The Basics with Applications. [S.1.]: McGraw-Hill, 1995. (McGraw-Hill International Editions: Mechanical Engineering). ISBN 9780071132107.

ANDREASEN, C. S. Topology optimization of inertia driven dosing units. Structural and Multidisciplinary Optimization, v. 55, n. 4, p. 1301-1309, Apr 2017.

ANDREASEN, C. S.; GERSBORG, A. R.; SIGMUND, O. Topology optimization of microfluidic mixers. International Journal for Numerical Methods in Fluids, v. 61, n. 5, p. 498-513, 2009.

ATALAN, A. Is the lockdown important to prevent the covid-19 pandemic? Effects on psychology, environment and economy-perspective. Annals of Medicine and Surgery, v. 56, p. 38-42, Aug 2020.

BENCHARIF, M. et al. The benefit of droplet injection on the performance of an ejector refrigeration cycle working with r245fa. International Journal of Refrigeration, v. 113, p. 276-287, 2020.

BORRVALL, T.; PETERSSON, J. Topology optimization of fluids in stokes flow. International Journal for Numerical Methods in Fluids, v. 41, n. 1, p. 77-107, 2003.

BROWN, F. B. Discussion: "jet pump cavitation” (Cunningham, R. G., Hansen, A. G., and Na, T. Y., 1970, asme j. basic eng., 92, pp. 483-492). Journal of Basic Engineering, v. 92, n. 3, p. 492-492, Sep 1970.

CACUCI, D. G. et al. Sensitivity theory for general systems of nonlinear equations. Nuclear Science and Engineering, v. 75, p. 88-110, 1980.

CAIRNS, J. R.; NA, T. Y. Optimum design of water jet pumps. Journal of Engineering for Power, v. 91, n. 1, p. 62-68, Jan 1969.

ÇENGEL, Y. A.; CIMBALA, J. M. Fluid Mechanics: Fundamentals and Applications. 3rd. ed. [S.1.]: McGraw-Hill, 2013. 
CHALLIS, V. J.; GUEST, J. K. Level-set topology optimization of fluids in Stokes flow. International Journal for Numerical Methods in Engineering, v. 79, n. 10, p. 1284-1308, 2009.

CHAPMAN, L. Transport and climate change: a review. Journal of Transport Geography, v. 15, n. 5, p. 354-367, set. 2007.

CHEN, J. et al. Performance analysis of a novel organic rankine cycle with a vapor-liquid ejector. Energy Conversion and Management, v. 157, p. 382â395, 2018.

CHEN, W. et al. Numerical assessment of ejector performance enhancement by means of two-bypass inlets. Applied Thermal Engineering, v. 171, p. 115086, 2020.

COMSOL. COMSOL Multiphysics Reference Manual, version 5.5. [S.1.], 2019.

COOK, J. et al. Quantifying the consensus on anthropogenic global warming in the scientific literature. Environmental Research Letters, IOP Publishing, v. 8, n. 2, p. 024024, May 2013.

CUAMATZI-MELENDEZ, R.; FLORES-CUAMATZI, E. Modelling fluid-structure interaction of water recirculating flow to predict damage and/or failure in a jet-pump assembly of a nuclear boiling water reactor. Engineering Structures, v. 206, p. 110155, 2020.

CUNNINGHAM, R.; DOPKIN, R. Jet breakup and mixing throat lengths for the liquid jet gas pump. 1974.

CUNNINGHAM, R. G. Jet-pump theory and performance with fluids of high viscosity. American Society of Mechanical Engineers, v. 79, p. 1807-1820, 1957.

DEDE, E. M. Multiphysics topology optimization of heat transfer and fluid flow systems. In: in Proceedings of the COMSOL Users Conference. [S.1.: s.n.], 2009.

DEHGHANI, T. et al. Mixing enhancement through a micromixer using topology optimization. Chemical Engineering Research and Design, v. 161, p. 187-196, Sep 2020.

DILGEN, C. B. et al. Topology optimization of turbulent flows. Computer Methods in Applied Mechanics and Engineering, v. 331, p. 363-393, Apr 2018.

DILGEN, S. B. et al. Density based topology optimization of turbulent flow heat transfer systems. Structural and Multidisciplinary Optimization, v. 57, n. 5, p. 1905-1918, May 2018 .

DOBSON, A. P. et al. Ecology and economics for pandemic prevention. Science, American Association for the Advancement of Science, v. 369, n. 6502, p. 379-381, 2020.

DONG, J. et al. Numerical investigation on the influence of mixing chamber length on steam ejector performance. Applied Thermal Engineering, v. 174, p. 115204, 2020.

DVORÁK, V. Shape optimization of axisymmetric ejector. In: . [S.1.: s.n.], 2006.

EBDALE, B. Capabilities and limitations of pumps. Steam jet ejectors and liquid ring pumps. Vacuum, v. 28, p. 337-341, 1978.

ENGINEERING SCIENCES DATA UNIT. Ejectors and Jet Pumps. Design and Performance for Incompressible Liquid Flow. [S.1.], 1985. 
ERMOLIEV, Y.; GAIVORONSKI, A. On Optimization of Discontinuous Systems. IIASA, Laxenburg, Austria, 1991.

FAN, Y. et al. Bat coronaviruses in China. Viruses, Multidisciplinary Digital Publishing Institute, v. 11, n. 3, p. 210, 2019.

GERSBORG-HANSEN, A.; SIGMUND, O.; HABER, R. B. Topology optimization of channel flow problems. Structural and Multidisciplinary Optimization, v. 30, n. 3, p. 181-192, Sep 2005.

GIANNAKOGlOU, K. C.; PAPADIMITRIOU, D. I. Adjoint Methods for Shape Optimization. Berlin, Heidelberg: Springer Berlin Heidelberg, 2008. 79-108 p.

GILES, M. B.; PIERCE, N. A. An introduction to the adjoint approach to design. Flow, turbulence and combustion, Springer, v. 65, n. 3-4, p. 393-415, 2000.

GUILLAUME, D. W.; JUDGE, T. A. Improving the efficiency of a jet pump using an elliptical nozzle. Review of Scientific Instruments, v. 70, n. 12, p. 4727-4729, 1999.

GUILLAUME, D. W.; JUDGE, T. A. Improving the efficiency of a jet pump using a swirling primary jet. Review of Scientific Instruments, v. 75, n. 2, p. 553-555, 2004.

HAERTEL, J. H. et al. Topology optimization of a pseudo 3d thermofluid heat sink model. International Journal of Heat and Mass Transfer, v. 121, p. 1073-1088, 2018.

HAUKE, G.; HUGHES, T. A unified approach to compressible and incompressible flows. Computer Methods in Applied Mechanics and Engineering, v. 113, n. 3, p. $389-395$, 1994.

HOLLAND, J. H. et al. Adaptation in natural and artificial systems: an introductory analysis with applications to biology, control, and artificial intelligence. [S.1.]: MIT press, 1992.

HÖRNLEIN, H. R.; SCHNITTKOWSKI, K. Software systems for structural optimization. [S.1.]: Birkhäuser, 2013. v. 110.

HYAFIL, A.; MORIÑA, D. Analysis of the impact of lockdown on the reproduction number of the SARS-Cov-2 in Spain. Gaceta Sanitaria, May 2020.

IPCC. Climate change 2014: impacts, adaptation, and vulnerability. Part B: regional aspects. Contribution of Working Group II to the Fifth Assessment Report of the Intergovernmental Panel on Climate Change. Cambridge, United Kingdom and New York, NY, USA, 2014.

ISMAGILOV, A. R.; SPIRIDONOV, E. K.; BELKINA, O. V. Liquid jet ejector efficiency improvement. Procedia Engineering, v. 206, p. 99-106, 2017.

JAMESON, A. Optimum aerodynamic design using CFD and control theory. In: 12th Computational Fluid Dynamics Conference. [S.1.: s.n.], 1995.

JONES, K. E. et al. Global trends in emerging infectious diseases. Nature, v. 451, n. 7181, p. 990-993, Feb 2008. 
KANITKAR, T. The COVID-19 lockdown in India: Impacts on the economy and the power sector. Global Transitions, v. 2, p. 150-156, Jan 2020.

KARNIADAKIS, G.; SHERWIN, S. Spectral/hp element methods for computational fluid dynamics. [S.1.]: Oxford University Press, 2013.

KIM, S.; ALONSO, J.; JAMESON, A. A gradient accuracy study for the adjoint-based Navier-Stokes design method. In: 37th Aerospace Sciences Meeting and Exhibit. [S.l.: s.n.], 1999.

KOGA, A. A. et al. Development of heat sink device by using topology optimization. International Journal of Heat and Mass Transfer, v. 64, p. 759-772, 2013. ISSN 0017-9310.

LADYZHENSKAYA, O. A. The mathematical theory of viscous incompressible flow. [S.1.]: Gordon and Breach New York, 1969. v. 2.

LAZAROV, B. S.; SIGMUND, O. Filters in topology optimization based on Helmholtz-type differential equations. International Journal for Numerical Methods in Engineering, v. 86, n. 6, p. 765-781, 2011.

LIKNES, F. Jet Pump. Thesis (Master) - Norwegian University of Science and Technology, Trondheim, Norway, 2013.

LIU, F.; LI, D.; ZENG, X. Research on energy saving technology of distributing combined adjustable jet pump. Procedia Engineering, v. 205, p. 738 - 743, 2017. 10th International Symposium on Heating, Ventilation and Air Conditioning, ISHVAC2017, 19-22 October 2017, Jinan, China.

LIU, X.; ZHAN, Q.; LIA, Y. The application analysis of jet pump in heating system. Procedia Engineering, v. 205, p. 2208 - 2214, 2017. 10th International Symposium on Heating, Ventilation and Air Conditioning, ISHVAC2017, 19-22 October 2017, Jinan, China.

LONG, X.; HAN, N.; CHEN, Q. Influence of nozzle exit tip thickness on the performance and flow field of jet pump. Journal of Mechanical Science and Technology, v. 22, n. 10, p. 1959-1965, Oct 2008.

LONG, X. et al. Experimental investigation of the cavitation characteristics of jet pump cavitation reactors with special emphasis on negative flow ratios. Experimental Thermal and Fluid Science, v. 96, p. $33-42,2018$.

LONG, X. et al. An experimental study of cavitation damage on tissue of Carassius auratus in a jet fish pump. Ocean Engineering, v. 174, p. 43 - 50, 2019.

MALISKA, C. R. Transferência de calor e mecânica dos fluidos computacional . [S.1.]: Grupo Gen-LTC, 2017.

MANGIALARDO, A. et al. Numerical investigation on a jet pump evolving liquid lead for GEN-IV reactors. Nuclear Engineering and Design, v. 280, p. 608â618, 2014.

MEHTA, S. P. A study of water jet pumps. Thesis (Master) - Kansas State University, Kansas, EUA, 1968. 
MITROPOULOU, C. C. et al. 4 - evolution strategies-based metaheuristics in structural design optimization. In: GANDOMI, A. H. et al. (Ed.). Metaheuristic Applications in Structures and Infrastructures. Oxford: Elsevier, 2013. p. 79 - 102.

MUELLER, N. H. G. Water jet pump. Journal of the Hydraulics Division, ASCE, v. 90, n. 3, p. $83-113,1964$.

NARABAYASHI, T. et al. Flow analysis for single and multi-nozzle jet pump. JSME International Journal Series B, Fluids and Thermal Engineering, Japan, v. 49, n. 4, p. 933-940, 2006.

PAKENHAM, K. I. et al. The moderating roles of psychological flexibility and inflexibility on the mental health impacts of COVID-19 pandemic and lockdown in Italy. Journal of Contextual Behavioral Science, v. 17, p. 109-118, Jul 2020.

PAPOUTSIS-KIACHAGIAS, E. M.; GIANNAKOGLOU, K. C. Continuous adjoint methods for turbulent flows, applied to shape and topological optimization: Industrial applications. Archives of Computational Methods in Engineering, v. 23, p. 255-299, 2016.

PENG, Y. et al. Application and economic analysis of water jet pump in new district heating system. Procedia Engineering, v. 205, p. 996 - 1003, 2017. 10th International Symposium on Heating, Ventilation and Air Conditioning, ISHVAC2017, 19-22 October 2017, Jinan, China.

PICELLI, R.; SIVAPURAM, R.; XIE, Y. M. A 101-line matlab code for topology optimization using binary variables and integer programming. Structural and Multidisciplinary Optimization, v. 63, n. 2, p. 935-954, Feb 2021.

RANKINE, W. J. M. On the mathematical theory of combined streams. Proceedings of the Royal Society of London, v. 19, n. 123-129, p. 90-94, 1871.

REDDY, J. An introduction to the finite element method. [S.1.]: McGraw-Hill New York, 2004.

REDDY, J. N.; GARTLING, D. K. The finite element method in heat transfer and fluid dynamics. [S.1.]: CRC press, 2010.

REDDY, Y.; KAR, S. Theory and performance of water jet pump. Journal of the Hydraulics Division, ASCE, v. 94, n. 5, p. 1261-1282, 1968.

REIS, L. B.; GIORIA, R. dos S. Optimization of liquid jet ejector geometry and its impact on flow fields. Applied Thermal Engineering, v. 194, p. 117132, 2021.

RUANGTRAKOON, N.; THONGTIP, T. An experimental investigation to determine the optimal heat source temperature for R141b ejector operation in refrigeration cycle. Applied Thermal Engineering, v. 170, p. 114841, Apr 2020.

SANGER, N. L. Noncavitating and cavitating performance of two low-area-ratio water jet pumps with throat lengths of 5.66 diameters. Washington, D.C., USA, 1968.

SCHULZ, F.; FASOL, K. H. Wasserstrahlpumpen zur Förderung von Flüssigkeiten. 1. ed. [S.1.]: Springer-Verlag Wien, 1958.

SILVESTER, R. Water jet pump - its uses in hydroeletric schemes. Water Power, v. 12, p. 176, 1960. 
STEPANOFF, A. J. Centrifugal and Axial Flow Pumps: Theory, Design, and Application. 2nd edition. ed. New York: Krieger Publishing Company, 1957.

SUN, D.-W. Comparative study of the performance of an ejector refrigeration cycle operating with various refrigerants. Energy Conversion and Management, v. 40, n. 8, p. 873-884, 1999.

SUN, S.; LIEBERSBACH, P.; QIAN, X. 3D topology optimization of heat sinks for liquid cooling. Applied Thermal Engineering, v. 178, p. 115540, Sep 2020.

SURYANTO, S.; HAMZAH, N.; TAUFIK, A. The novel vacuum drying using the steam ejector. Drying Technology, Taylor Francis, v. 39, n. 7, p. 905-911, 2021.

SVANBERG, K. The method of moving asymptotes - a new method for structural optimization. International Journal for Numerical Methods in Engineering, v. 24, n. 2, p. 359-373, 1987.

SVANBERG, K. A class of globally convergent optimization methods based on conservative convex separable approximations. Society for Industrial and Applied Mathematics, v. 12, n. 2, p. 555-573, 2002.

TAWK, R.; GHANNAM, B.; NEMER, M. Topology optimization of heat and mass transfer problems in two fluids - one solid domains. Numerical Heat Transfer, Part B: Fundamentals, Taylor Francis, v. 76, n. 3, p. 130-151, 2019.

THERIAULT, K. Nuclear Engineering Handbook. [S.1.]: CRC Press, 2016.

THOMSON, J. On a jet pump or apparatus for drawing-up water by power jet. 1852.

TOTEFF, J.; TOVAR, M. A. Design and multiparameter optimization of jet-pumps in a pipeline loops using CFD tools. In: AMERICAN SOCIETY OF MECHANICAL ENGINEERS. Fluids Engineering Division Summer Meeting. [S.1.], 2018. v. 51562, p. V002T09A025.

TROUT, T.; KEMPER, W. D.; AUST, R. Development and design of watercourse junction jet pumps. [S.1.], 1980.

URBAN, M. C. et al. Improving the forecast for biodiversity under climate change. American Association for the Advancement of Science, v. 353, n. 6304, 2016.

VERSTEEG, H. K.; MALALASEKERA, W. An Introduction to Computational Fluid Dynamics: The Finite Volume Method. 2nd. ed. [S.1.]: Pearson, 2007.

WANG, F.; LAZAROV, B. S.; SIGMUND, O. On projection methods, convergence and robust formulations in topology optimization. Structural and Multidisciplinary Optimization, v. 43, n. 6, p. 767-784, Jun 2011.

WANG, H.; WANG, H. Enhance hydraulic balance of a district cooling system with multiple jet pump. In: 10th International Conference on Applied Energy - ICAE2018. Hong Kong, China: [s.n.], 2018.

WANG, J. et al. Experimental investigation of cavity length pulsation characteristics of jet pumps during limited operation stage. Energy, v. 163, p. 61-73, 2018.

WINOTO, S.; LI, H.; SHAH, D. Efficiency of jet pumps. Journal of Hydraulic Engineering, v. 126, 022000. 
XU, M. et al. Impact of fish locomotion on the internal flow in a jet fish pump. Ocean Engineering, v. 187, p. 106227, 2019.

YAN, T. et al. Study on energy conservation water injection system of offshore platform based on jet pump. Journal of Petroleum Science and Engineering, v. 170, p. 368-373, 2018.

YAPICI, R.; ALDAS, K. Optimization of water jet pumps using numerical simulation. Proceedings of the Institution of Mechanical Engineers, Part A: Journal of Power and Energy, v. 227, n. 4, p. 438-449, 2013.

YIN, L. et al. What drives the vegetation dynamics in the Hengduan Mountain region, southwest China: Climate change or human activity? Ecological Indicators, v. 112, 2020.

YOON, G. H. Transient sensitivity analysis and topology optimization for particle motion in steady state laminar fluid. Computer Methods in Applied Mechanics and Engineering, v. 367, p. 113096, Aug 2020.

ZIENKIEWICZ, O. C.; MORGAN, K.; MORGAN, K. Finite elements and approximation. [S.1.]: Courier Corporation, 2006.

ZILLOBER, C. A globally convergent version of the method of moving asymptotes. Structural optimization, v. 6, n. 3, p. 166-174, Sep 1993. 


\section{APPENDIX A - GEOMETRY COMPARISON}

For sake of clearance, the comparison between the geometries resulting from optimization were presented in this appendix. The figures compares the results that uses the same Reynolds number and $\sigma$ tuning parameter differing only from the objective function used. The color red was assigned to the objective function $J_{1}$, to the $J_{2}$ blue and the objective function $J_{3}$ kept with the black color.

\section{A.1 Second geometry}

Figure 44 - Contours of the resulting geometry using the second geometry, Reynolds equal to 1 and $\sigma$ equal to 5 . The blue and black lines matches.

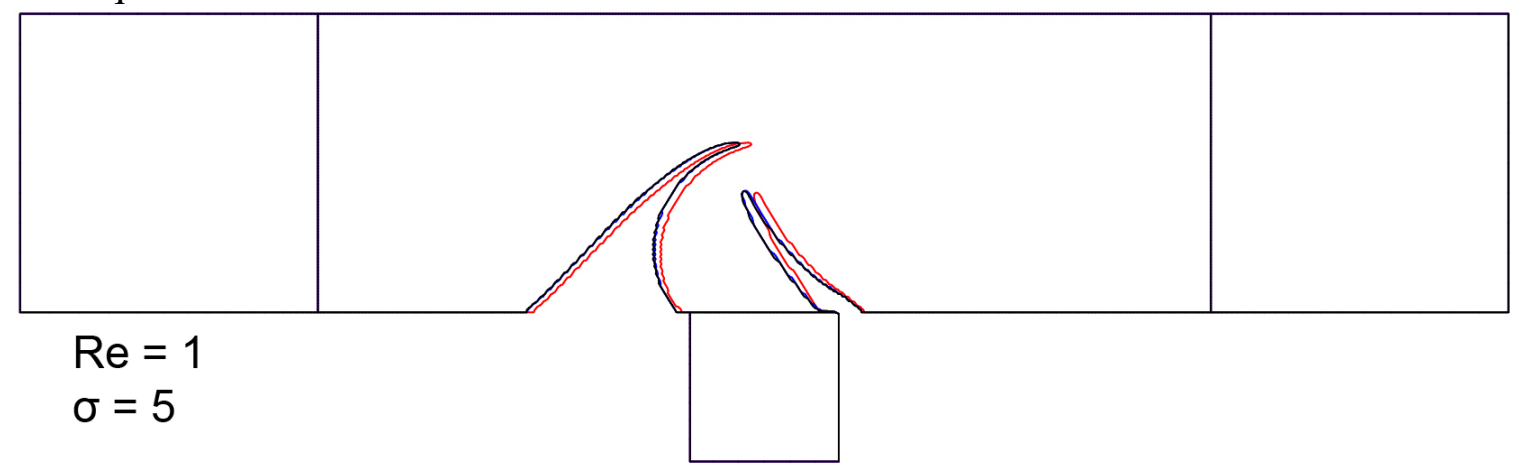

Source: Author. 
Figure 45 - Contours of the resulting geometry using the second geometry, Reynolds equal to 1 and $\sigma$ equal to 10 . The blue and black lines matches.

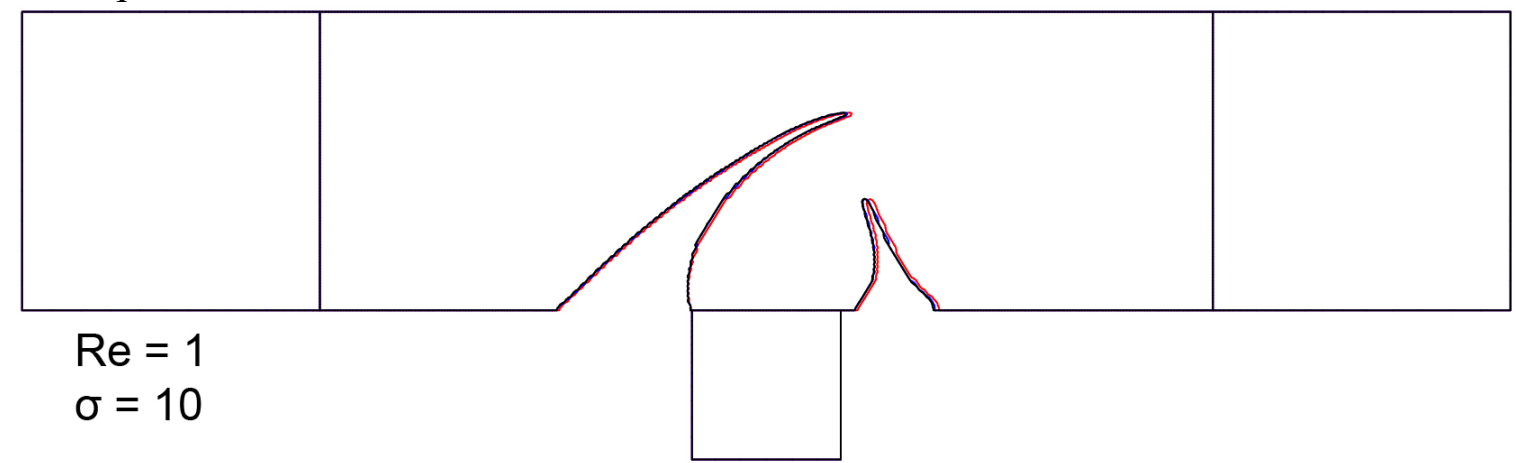

Source: Author.

Figure 46 - Contours of the resulting geometry using the second geometry, Reynolds equal to 1 and $\sigma$ equal to 30. The blue and black lines matches.

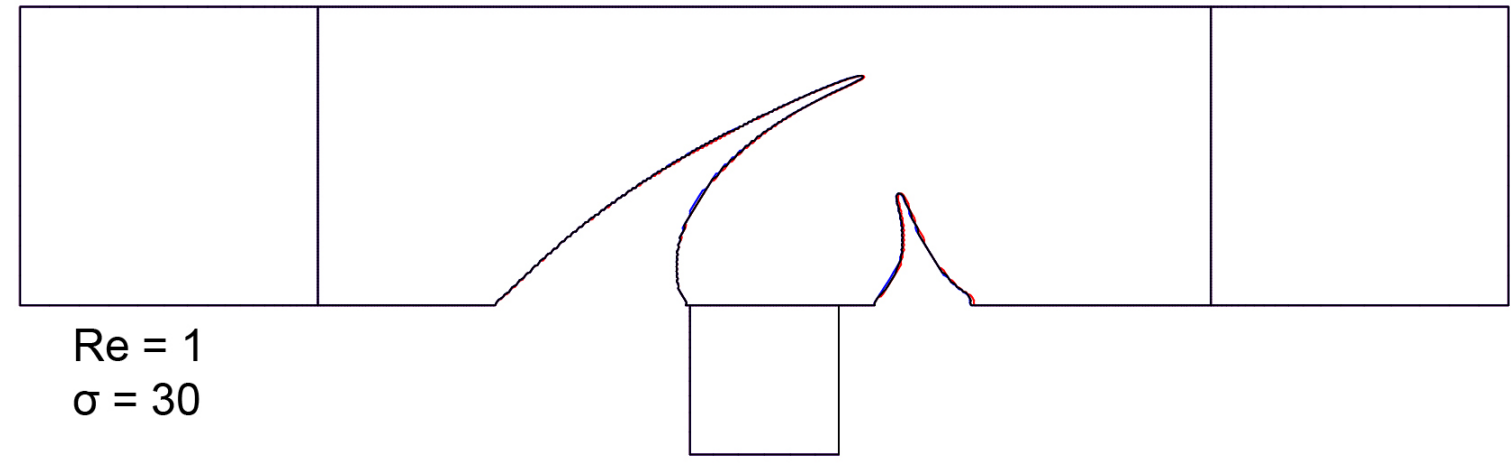

Source: Author.

Figure 47 - Contours of the resulting geometry using the second geometry, Reynolds equal to 10 and $\sigma$ equal to 5. The blue, black and red lines matches.

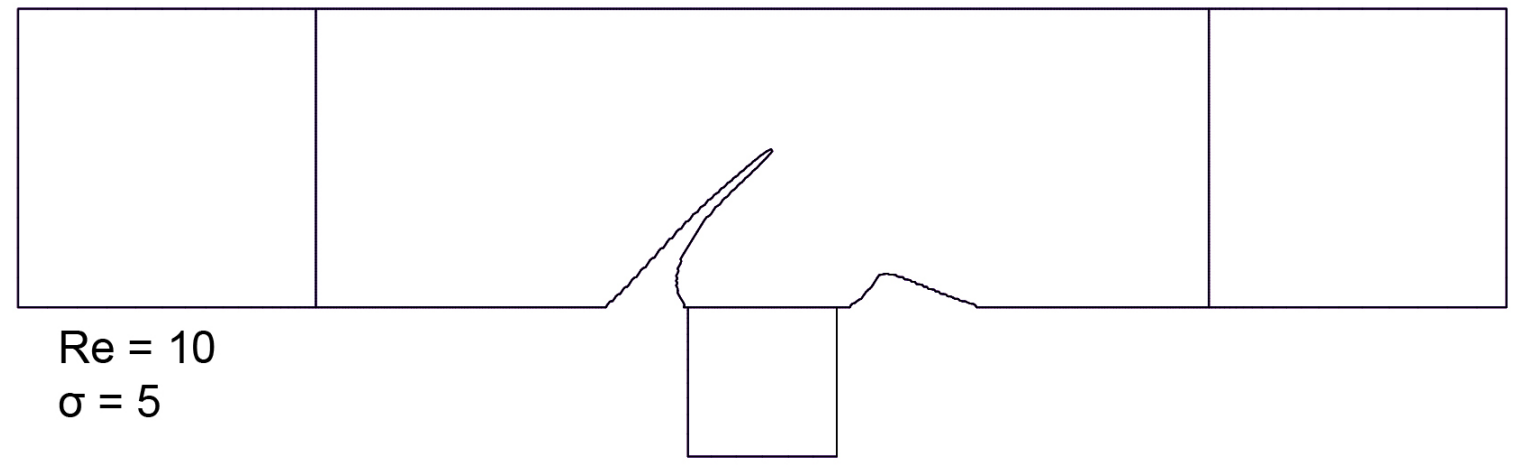

Source: Author. 
Figure 48 - Contours of the resulting geometry using the second geometry, Reynolds equal to 10 and $\sigma$ equal to 10 . The blue, black and red lines matches.

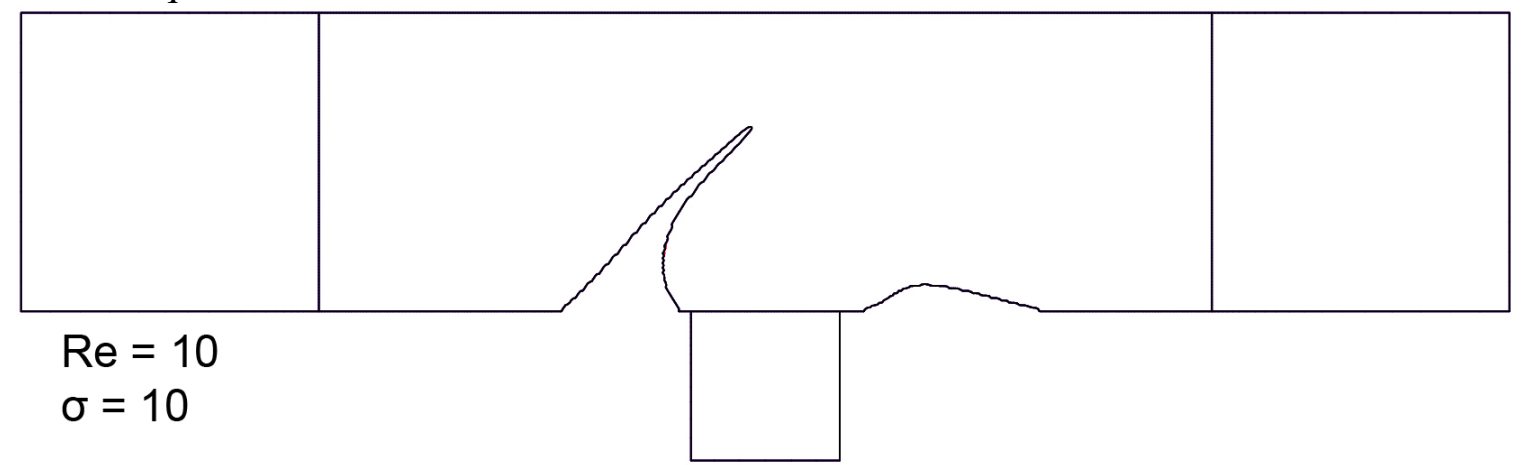

Source: Author.

Figure 49 - Contours of the resulting geometry using the second geometry, Reynolds equal to 10 and $\sigma$ equal to 30 . The blue, black and red lines matches.

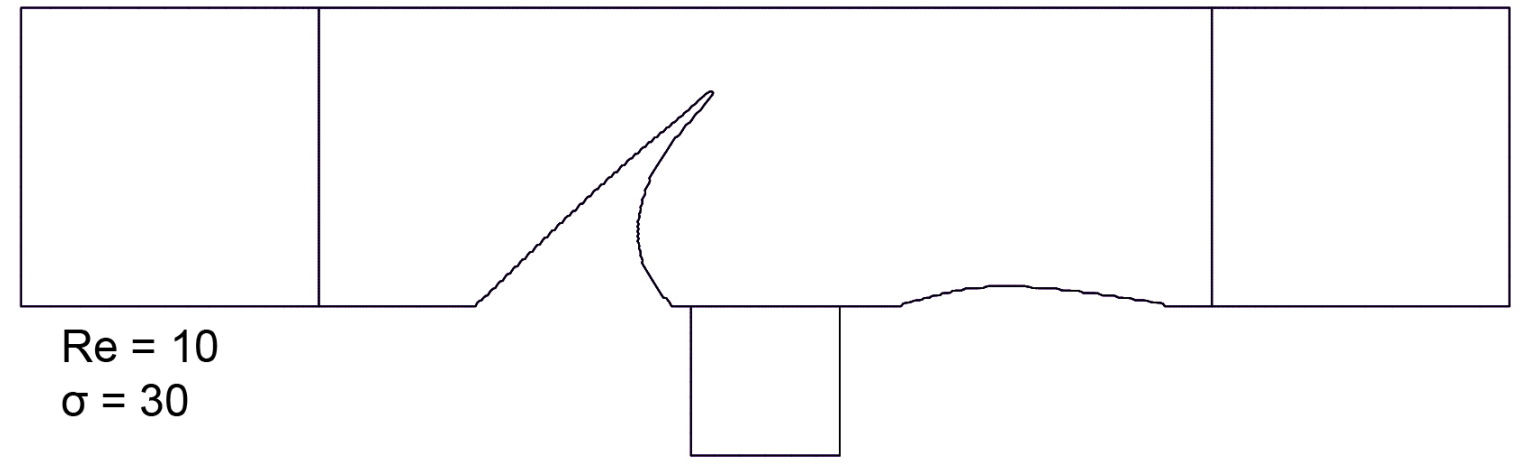

Source: Author.

Figure 50 - Contours of the resulting geometry using the second geometry, Reynolds equal to 30 and $\sigma$ equal to 5. The blue, black and red lines matches.

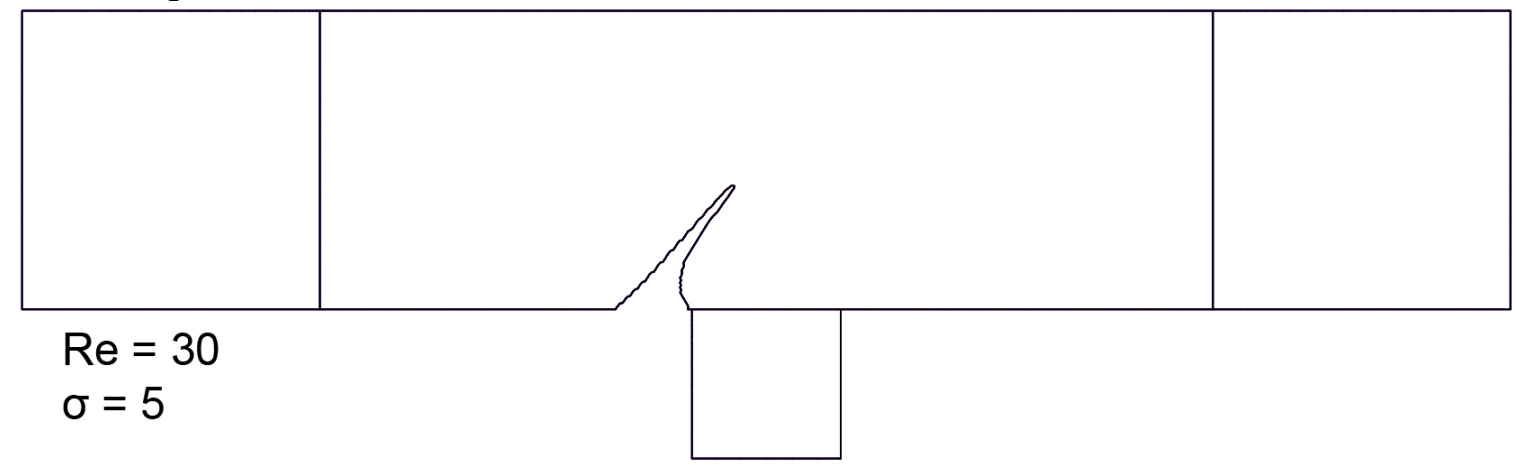

Source: Author. 
Figure 51 - Contours of the resulting geometry using the second geometry, Reynolds equal to 30 and $\sigma$ equal to 10 . The blue, black and red lines matches.

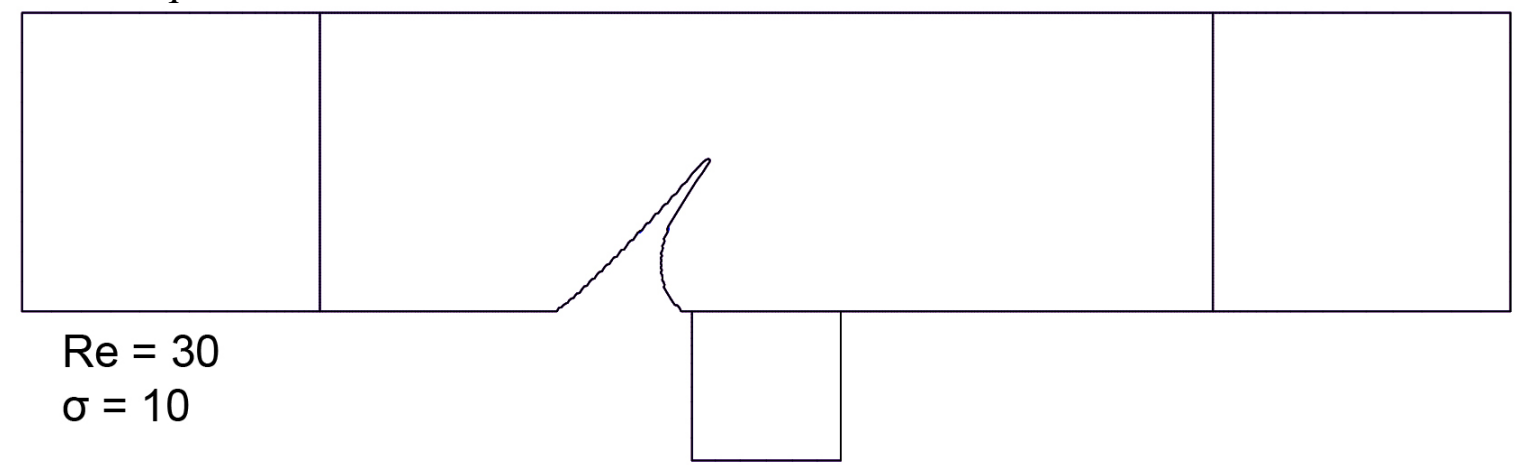

Source: Author.

Figure 52 - Contours of the resulting geometry using the second geometry, Reynolds equal to 30 and $\sigma$ equal to 30 . The blue, black and red lines matches.

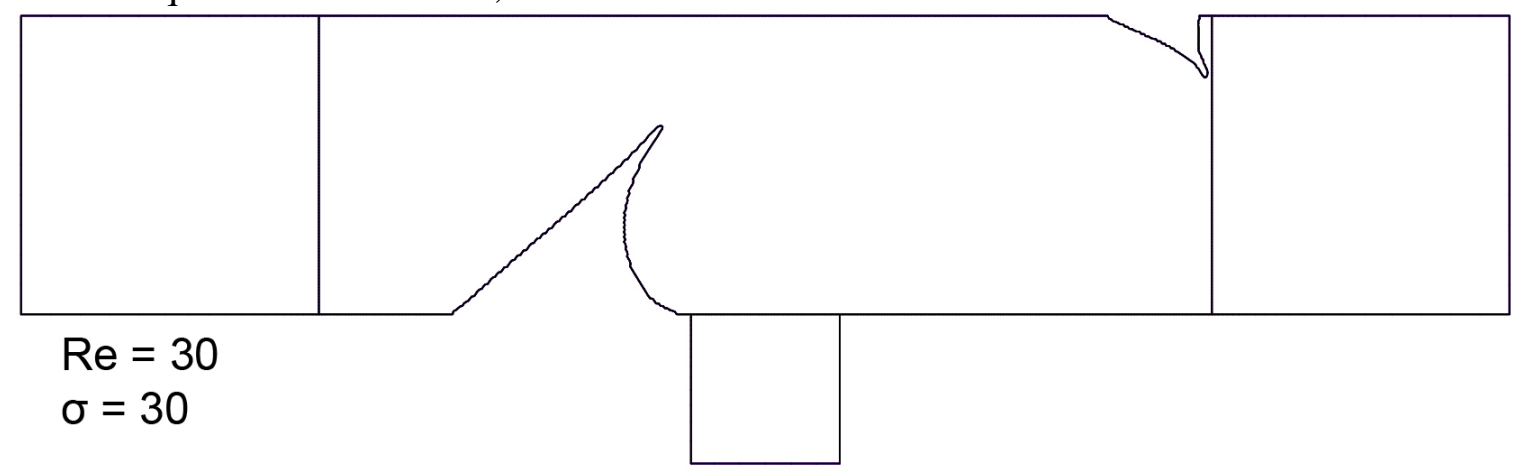

Source: Author.

\section{A.2 Third geometry}

Figure 53 - Contours of the resulting geometry using the third geometry, Reynolds equal to 1 and $\sigma$ equal to 5 . The blue and black lines matches. The red line slight diverges at the base of external wall.

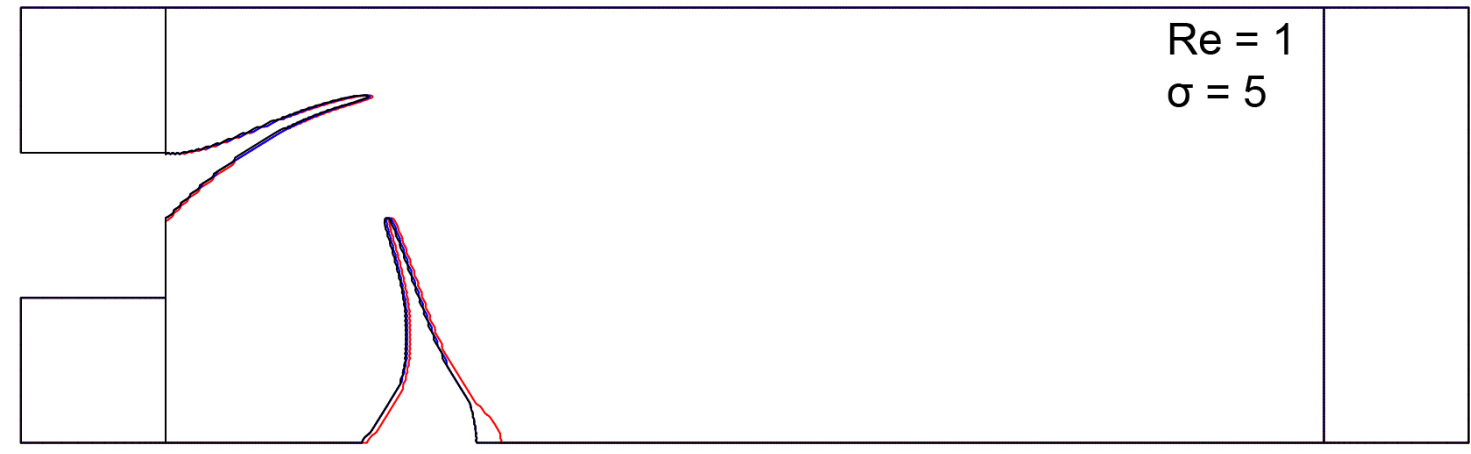

Source: Author. 
Figure 54 - Contours of the resulting geometry using the third geometry, Reynolds equal to 1 and $\sigma$ equal to 10 . The blue and black lines matches. The red line slight diverges at the base of external wall.

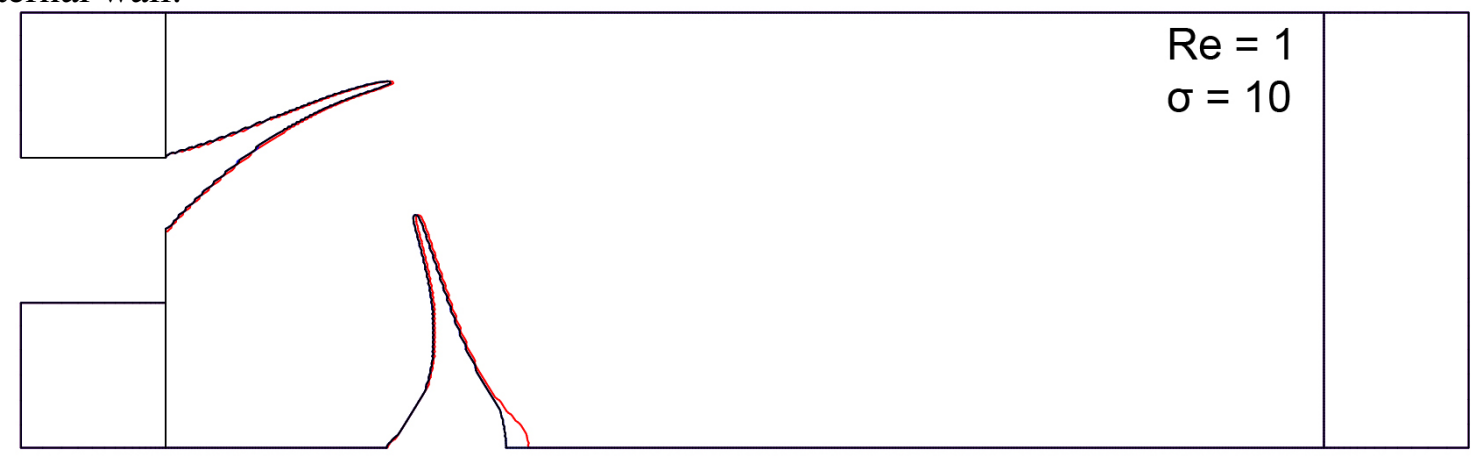

Source: Author.

Figure 55 - Contours of the resulting geometry using the third geometry, Reynolds equal to 1 and $\sigma$ equal to 5. The blue and black lines matches. The red line slight diverges at the base of external wall.

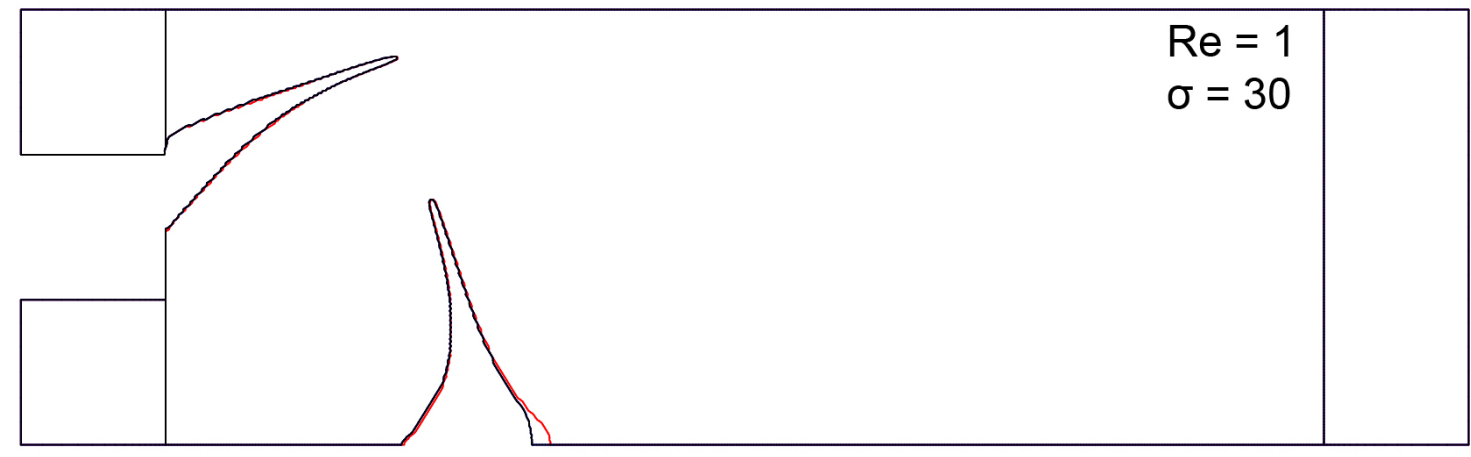

Source: Author.

Figure 56 - Contours of the resulting geometry using the third geometry, Reynolds equal to 10 and $\sigma$ equal to 5. The blue, black and red lines matches.

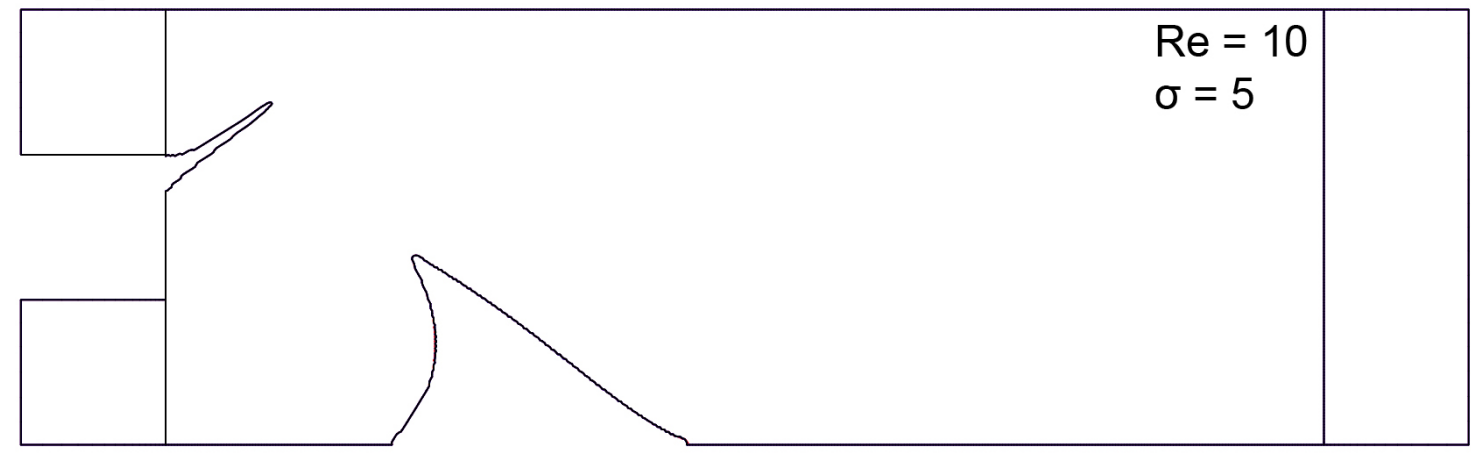

Source: Author. 
Figure 57 - Contours of the resulting geometry using the third geometry, Reynolds equal to 10 and $\sigma$ equal to 10 . The blue, black and red lines matches.

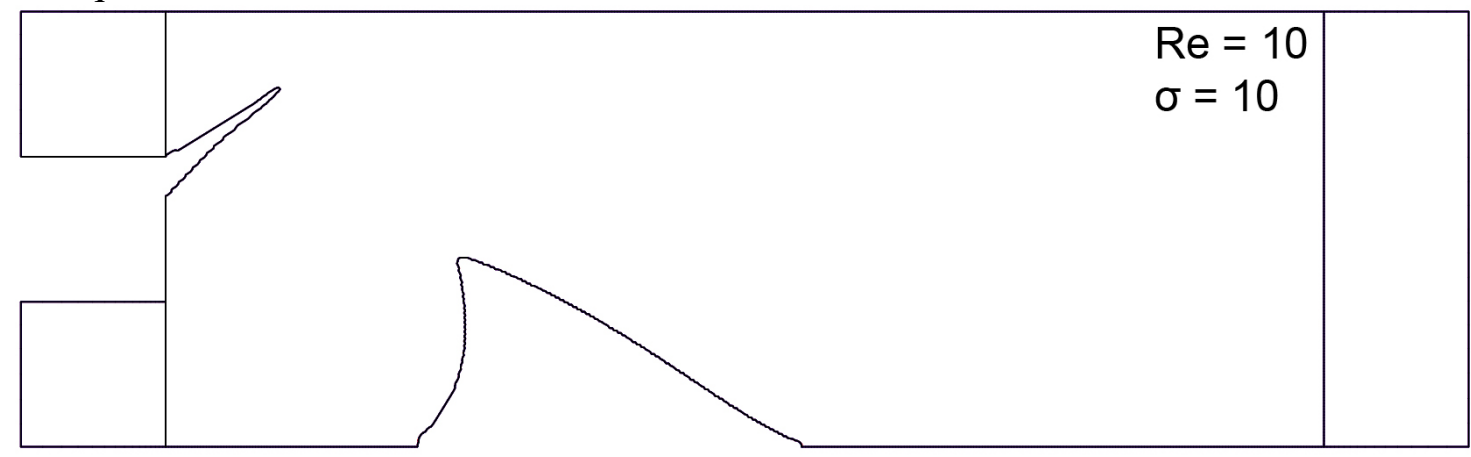

Source: Author.

Figure 58 - Contours of the resulting geometry using the third geometry, Reynolds equal to 10 and $\sigma$ equal to 30 . The blue, black and red lines matches.

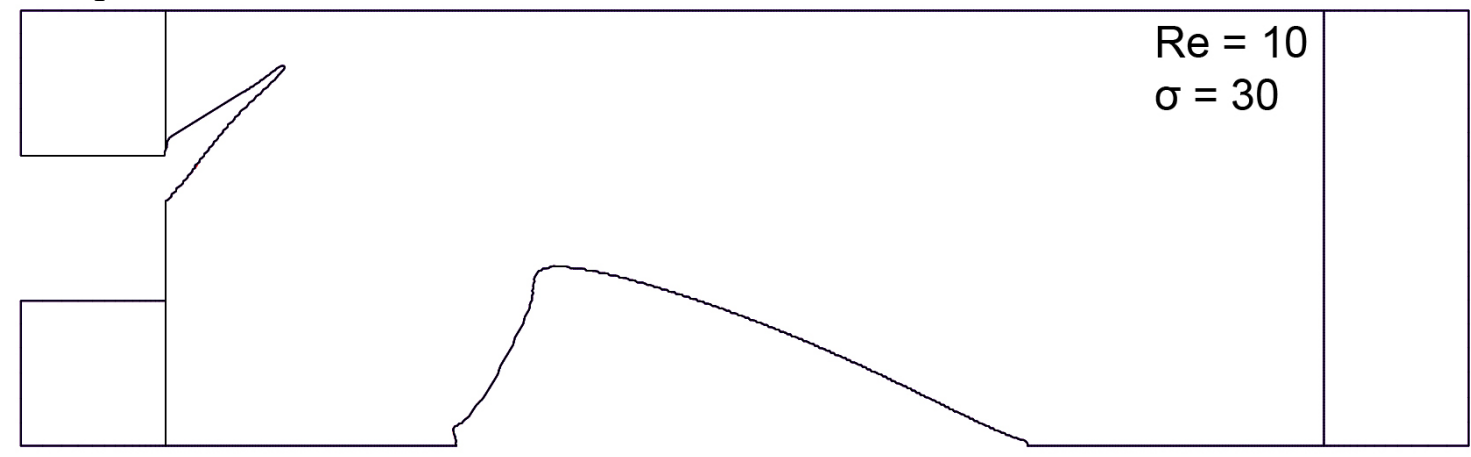

Source: Author.

Figure 59 - Contours of the resulting geometry using the third geometry, Reynolds equal to 30 and $\sigma$ equal to 5 . The blue, black and red lines matches.

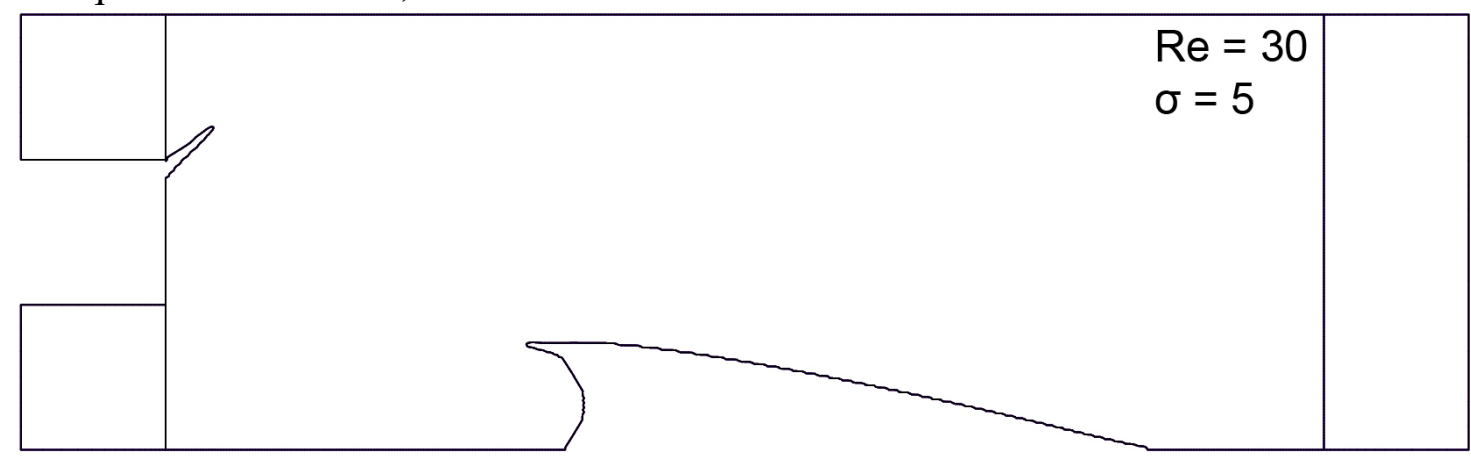

Source: Author. 
Figure 60 - Contours of the resulting geometry using the third geometry, Reynolds equal to 30 and $\sigma$ equal to 10 . The blue, black and red lines matches.

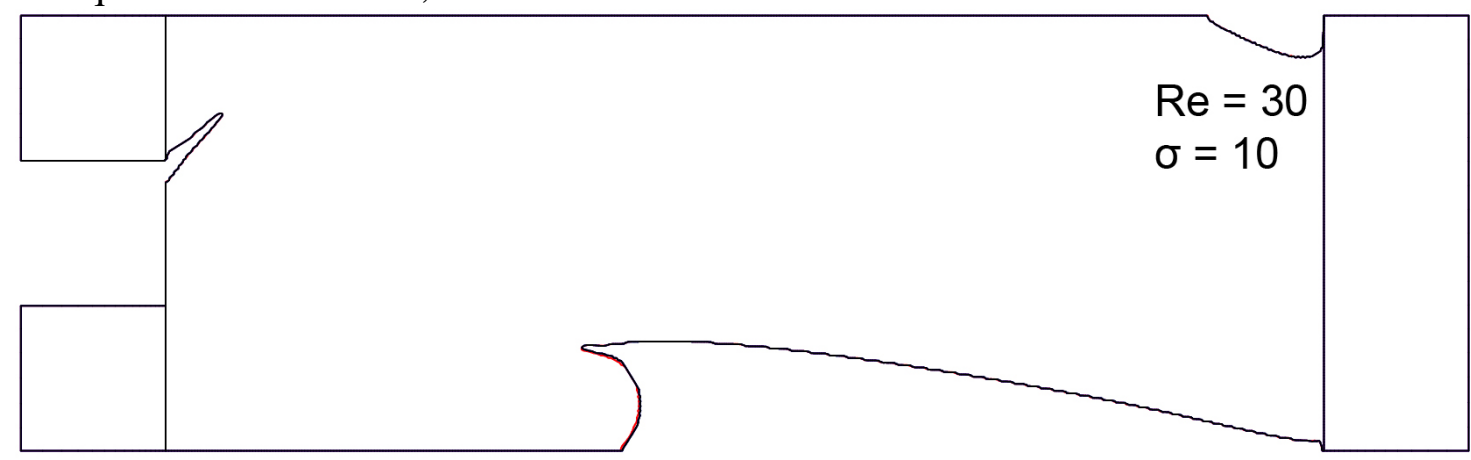

Source: Author.

Figure 61 - Contours of the resulting geometry using the third geometry, Reynolds equal to 30 and $\sigma$ equal to 30 . The blue, black and red lines matches.

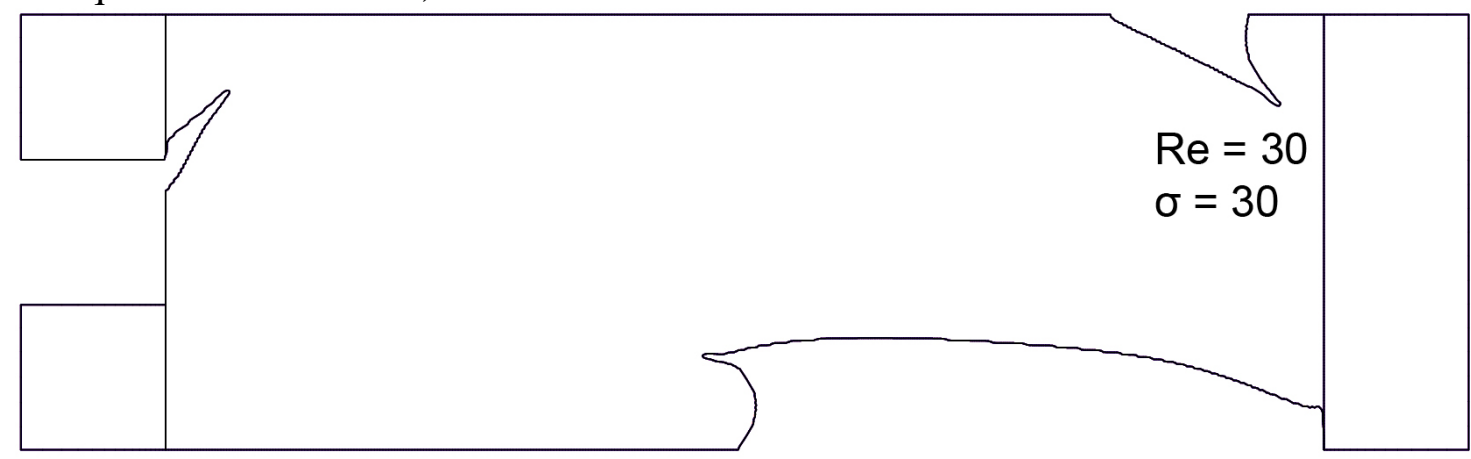

Source: Author. 


\section{APPENDIX B - FLOW FIELD}

For sake of clearance, the plot of the optimized domains using $J_{1}, J_{2}$ and $J_{3}$ were presented in this appendix.

Figure 62 - (a) Streamlines seed from the inlet (black) and secondary inlet (red) with the solid distribution (black structures), (b) velocity colour map and (c) static pressure contours with the solid distribution (black structures). Results obtained with Reynolds number equal to 1 and $\sigma$ equal to 5 .

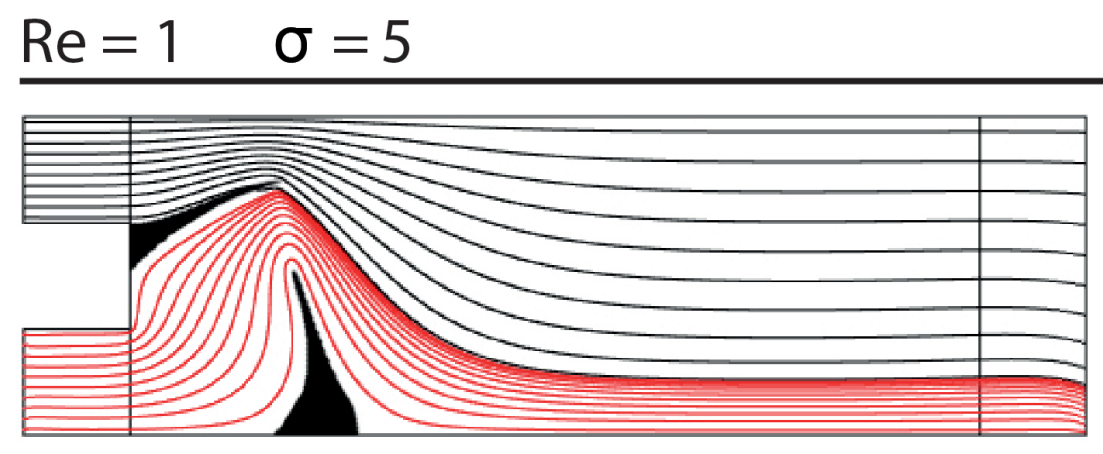

(a)

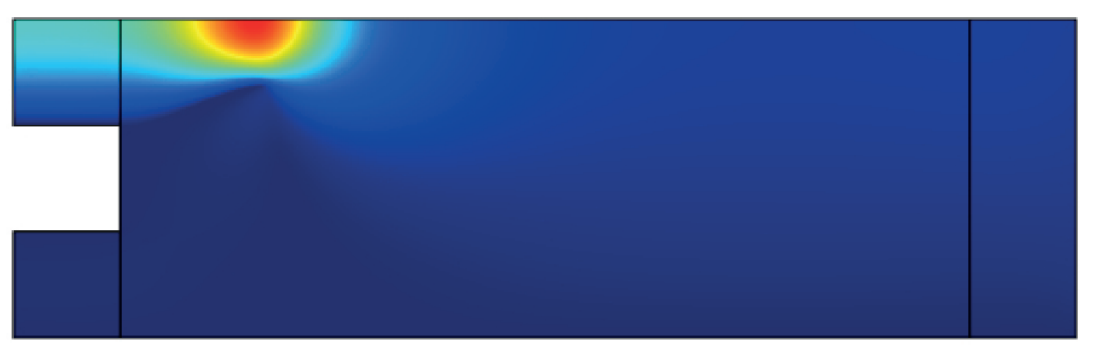

$\mathrm{m} / \mathrm{s}$

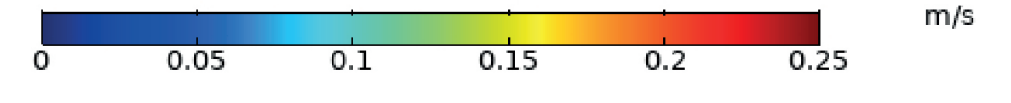

(b)

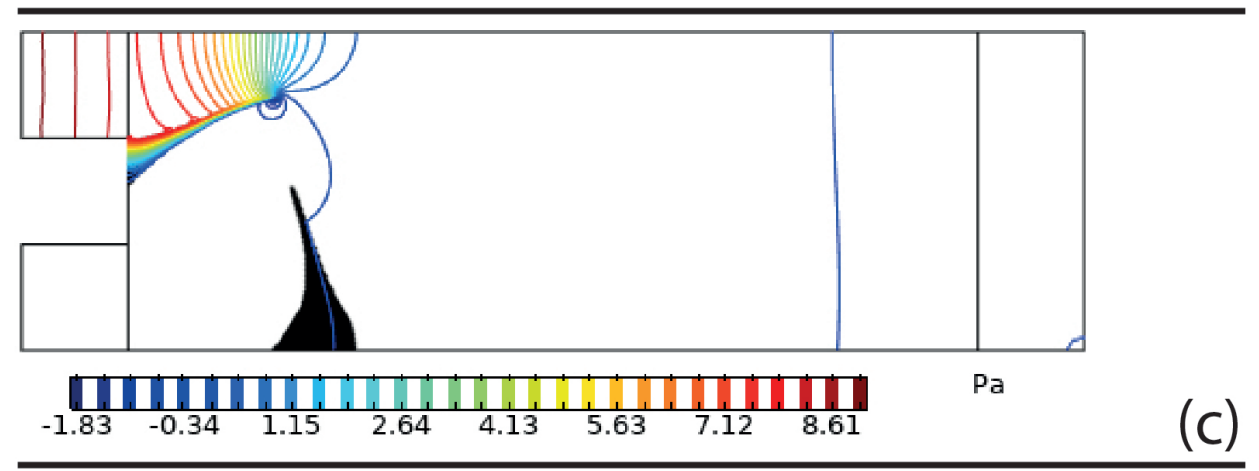

Source: Author. 
Figure 63 - (a) Stream lines seed from the inlet (black) and secondary inlet (red) with the solid distribution (black structures), (b) velocity colour map and (c) static pressure contours with the solid distribution (black structures). Results obtained with Reynolds number equal to 1 and $\sigma$ equal to 10.
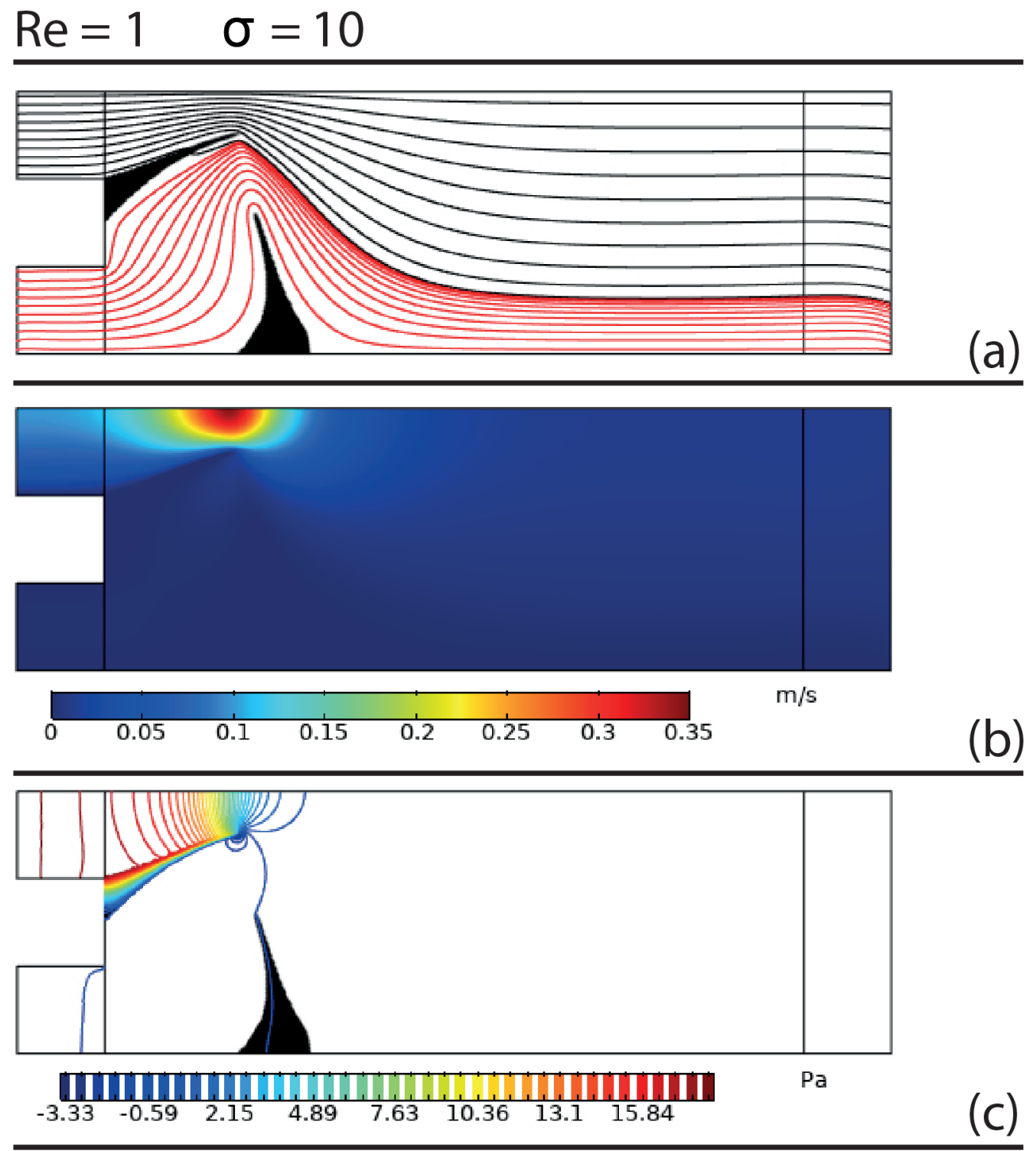

Source: Author. 
Figure 64 - (a) Stream lines seed from the inlet (black) and secondary inlet (red) with the solid distribution (black structures), (b) velocity colour map and (c) static pressure contours with the solid distribution (black structures). Results obtained with Reynolds number equal to 1 and $\sigma$ equal to 30.
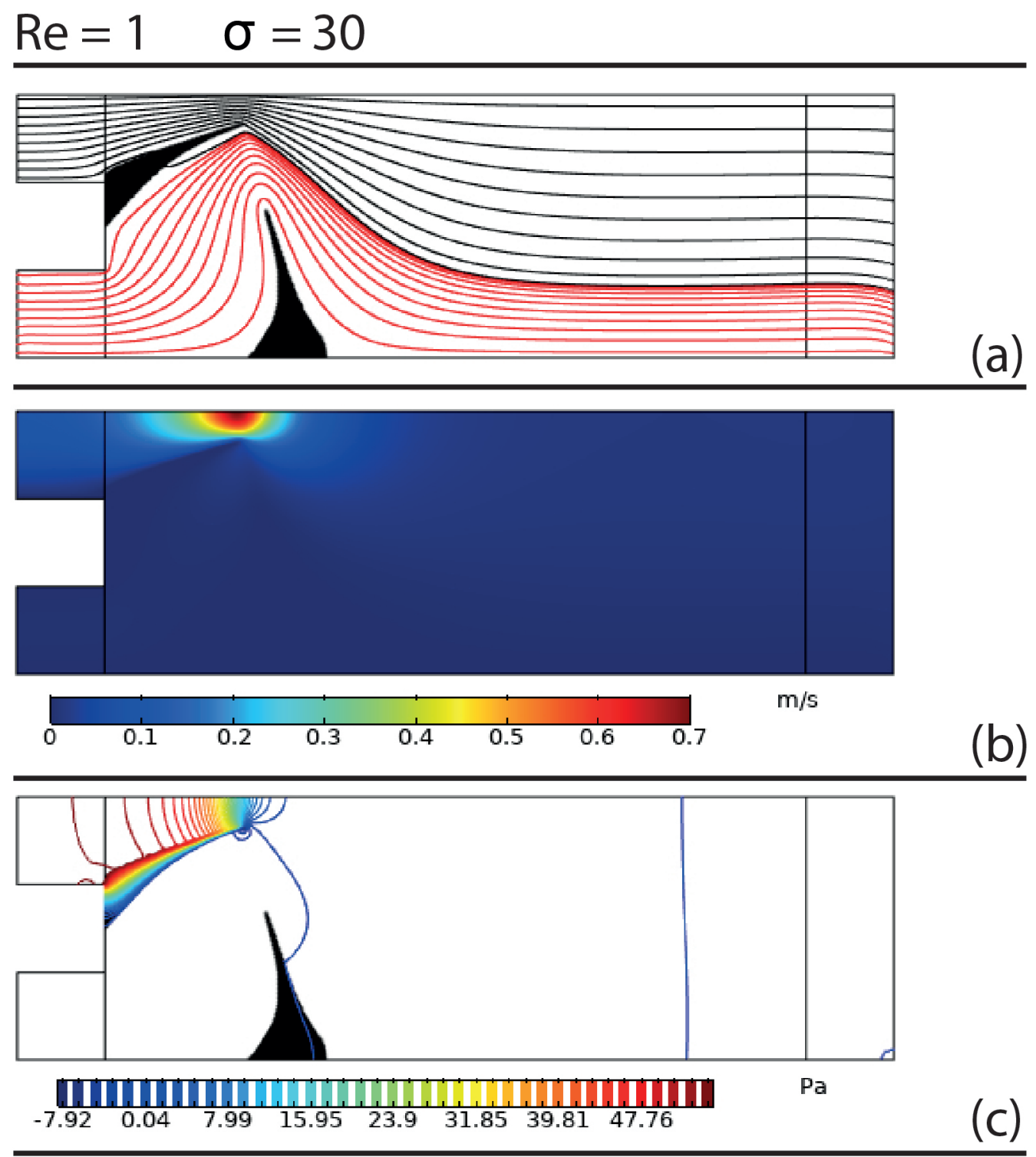

Source: Author. 
Figure 65 - (a) Stream lines seed from the inlet (black) and secondary inlet (red) with the solid distribution (black structures), (b) velocity colour map and (c) static pressure contours with the solid distribution (black structures). Results obtained with Reynolds number equal to 10 and $\sigma$ equal to 5 .

$$
\operatorname{Re}=10 \quad \sigma=5
$$
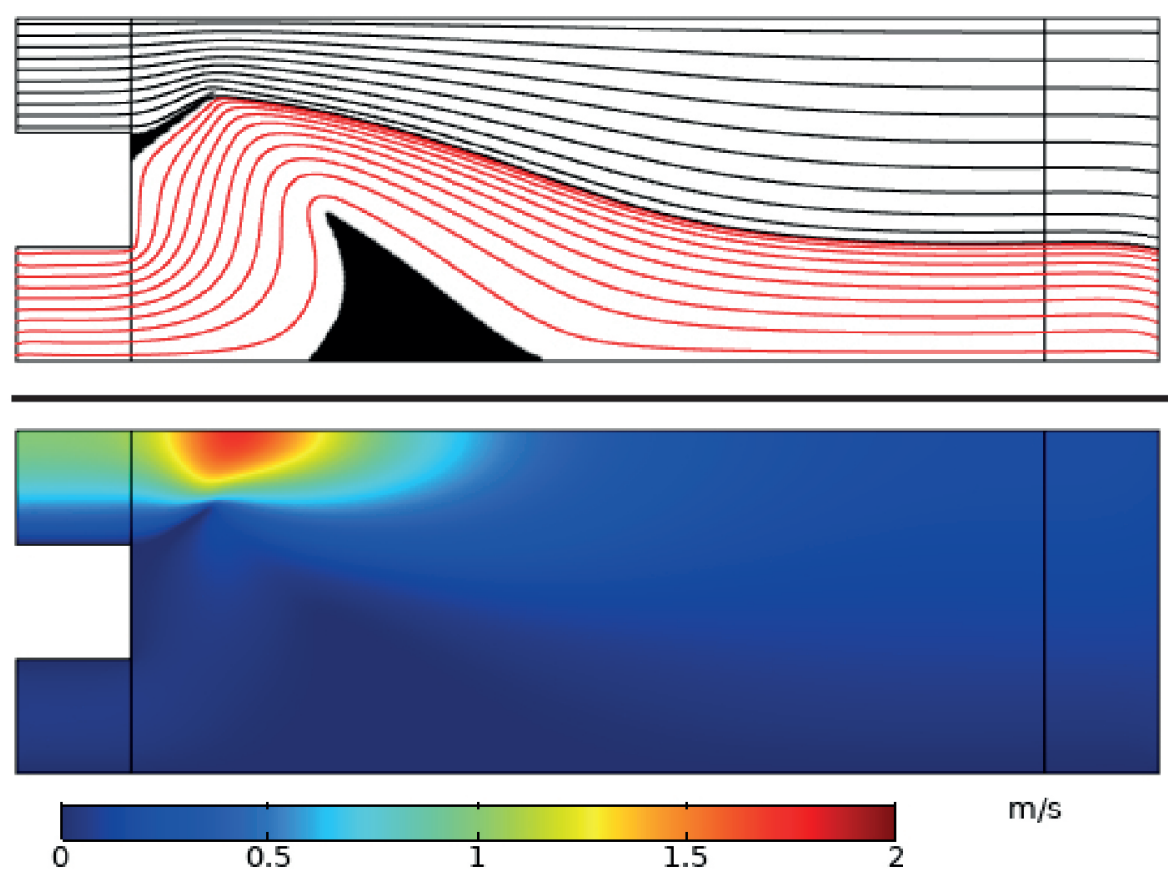

(b)

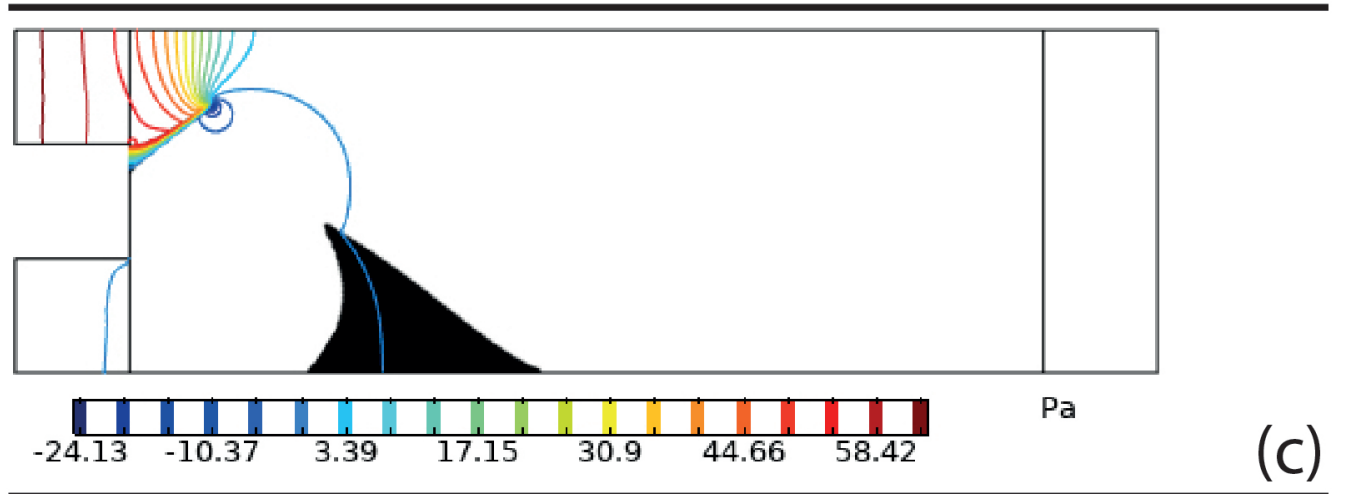

Source: Author. 
Figure 66 - (a) Stream lines seed from the inlet (black) and secondary inlet (red) with the solid distribution (black structures), (b) velocity colour map and (c) static pressure contours with the solid distribution (black structures). Results obtained with Reynolds number equal to 10 and $\sigma$ equal to 10.

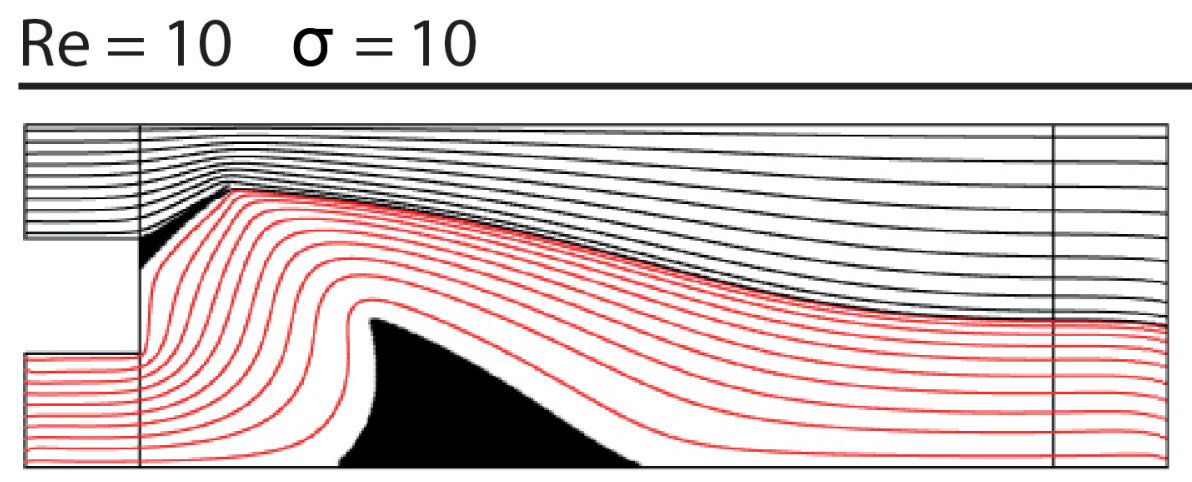

(a)
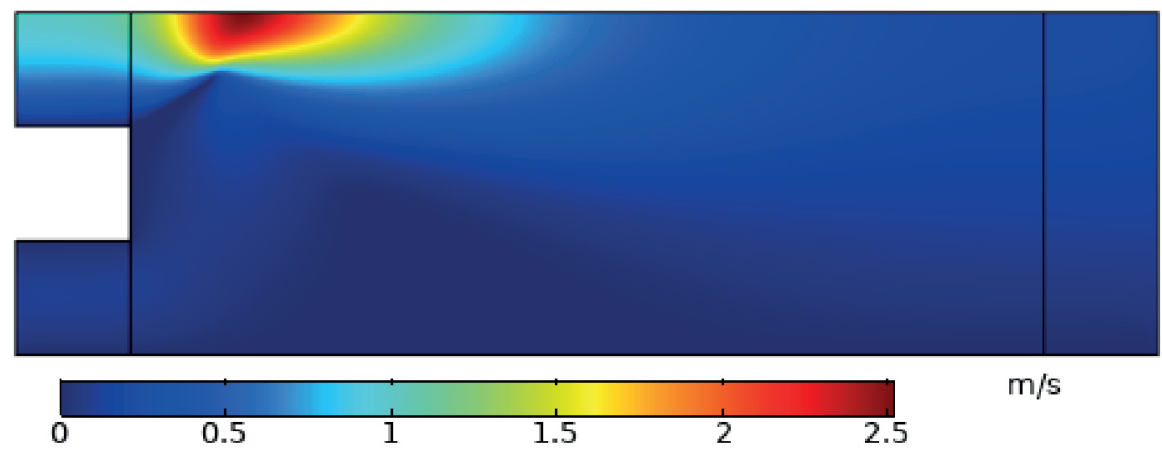

(b)

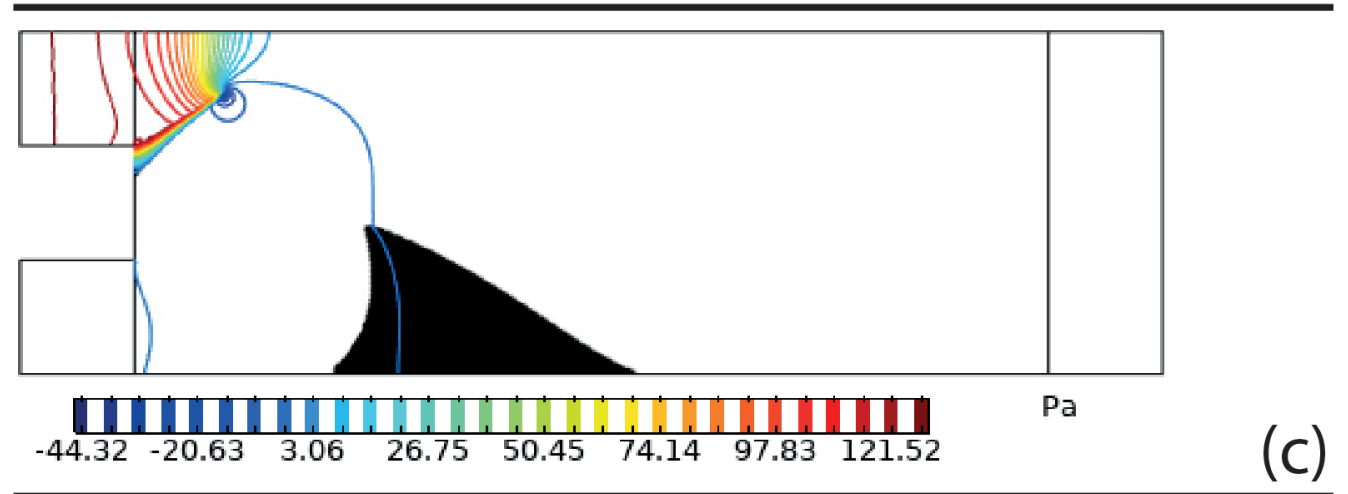

Source: Author. 
Figure 67 - (a) Stream lines seed from the inlet (black) and secondary inlet (red) with the solid distribution (black structures), (b) velocity colour map and (c) static pressure contours with the solid distribution (black structures). Results obtained with Reynolds number equal to 10 and $\sigma$ equal to 30 .

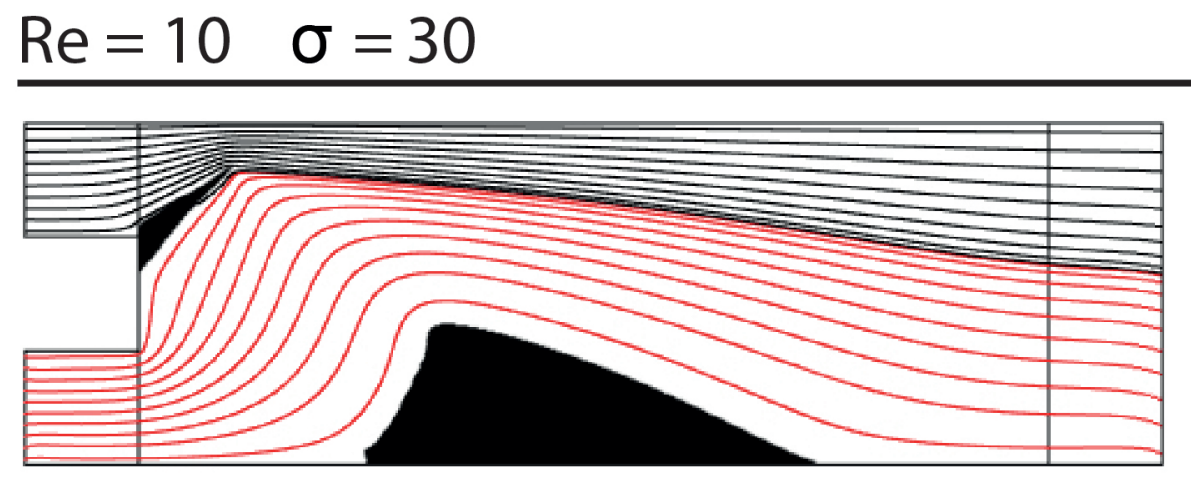

(a)
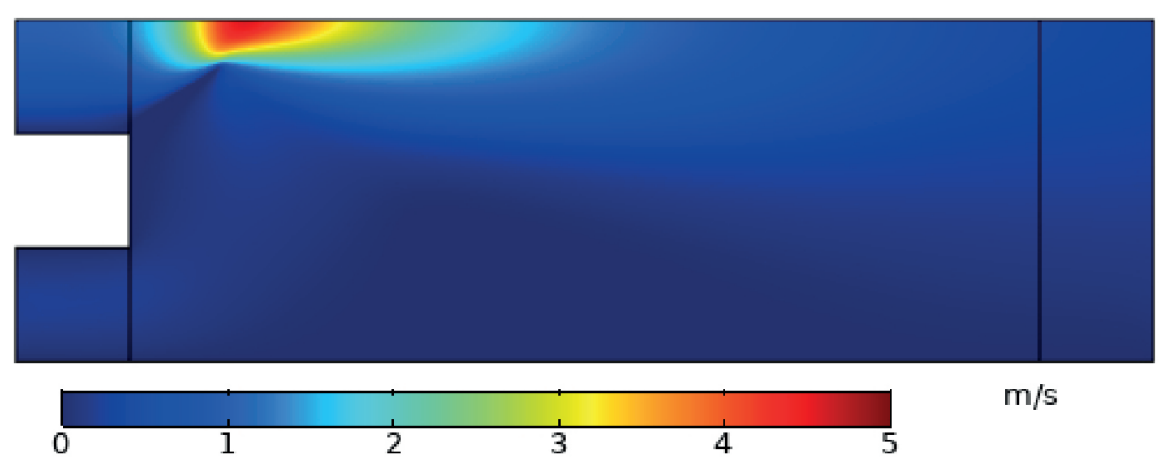

(b)

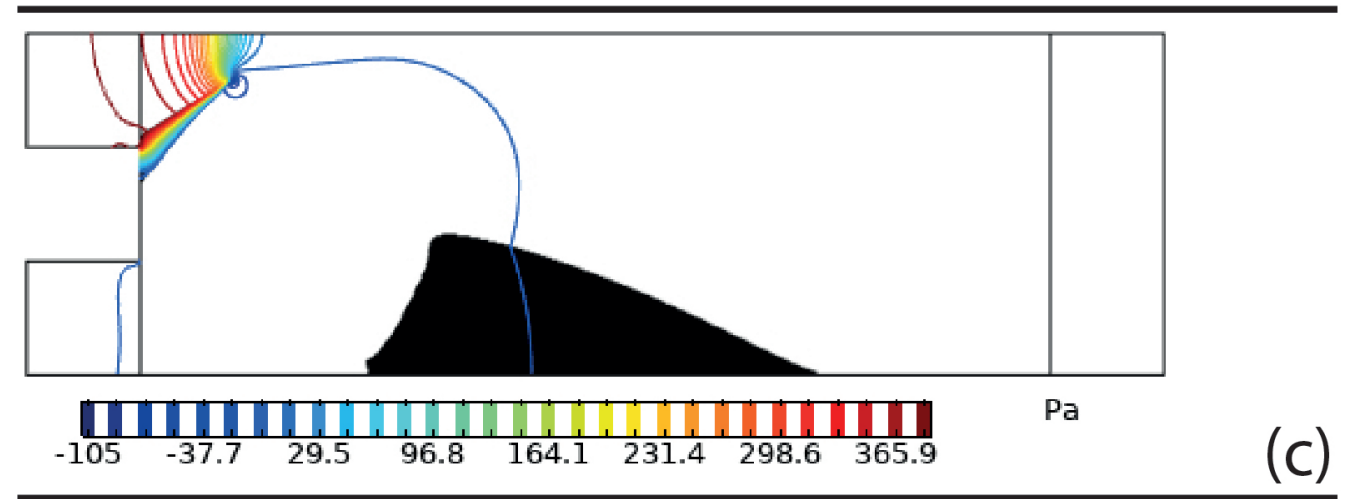

Source: Author. 
Figure 68 - (a) Stream lines seed from the inlet (black), secondary inlet (red) and recirculation zones (blue) with the solid distribution (black structures), (b) velocity colour map and (c) static pressure contours with the solid distribution (black structures). Results obtained with Reynolds number equal to 30 and $\sigma$ equal to 5 .
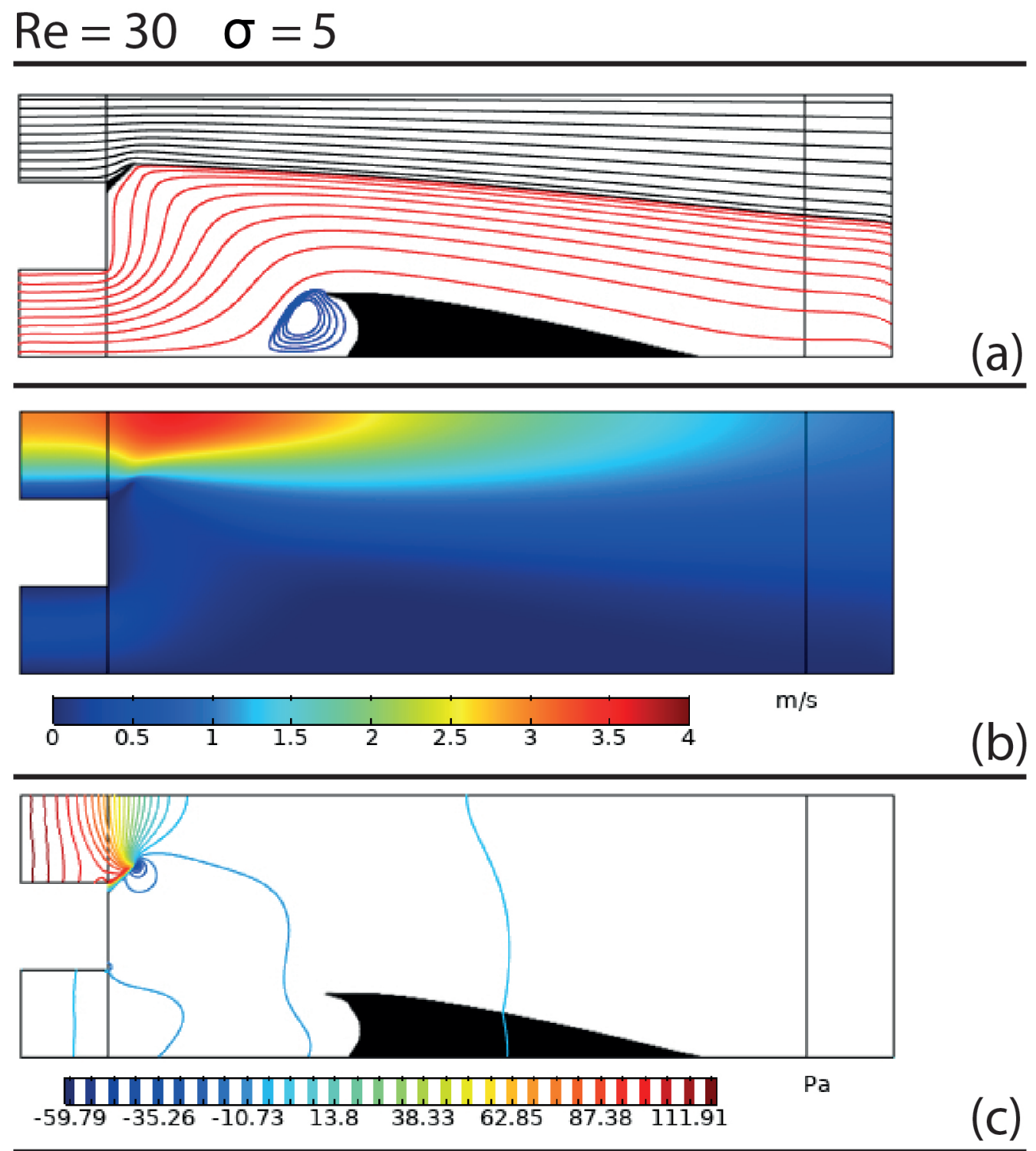

Source: Author. 
Figure 69 - (a) Stream lines seed from the inlet (black) and secondary inlet (red) and recirculation zones (blue) with the solid distribution (black structures), (b) velocity colour map and (c) static pressure contours with the solid distribution (black structures). Results obtained with Reynolds number equal to 30 and $\sigma$ equal to 10 .
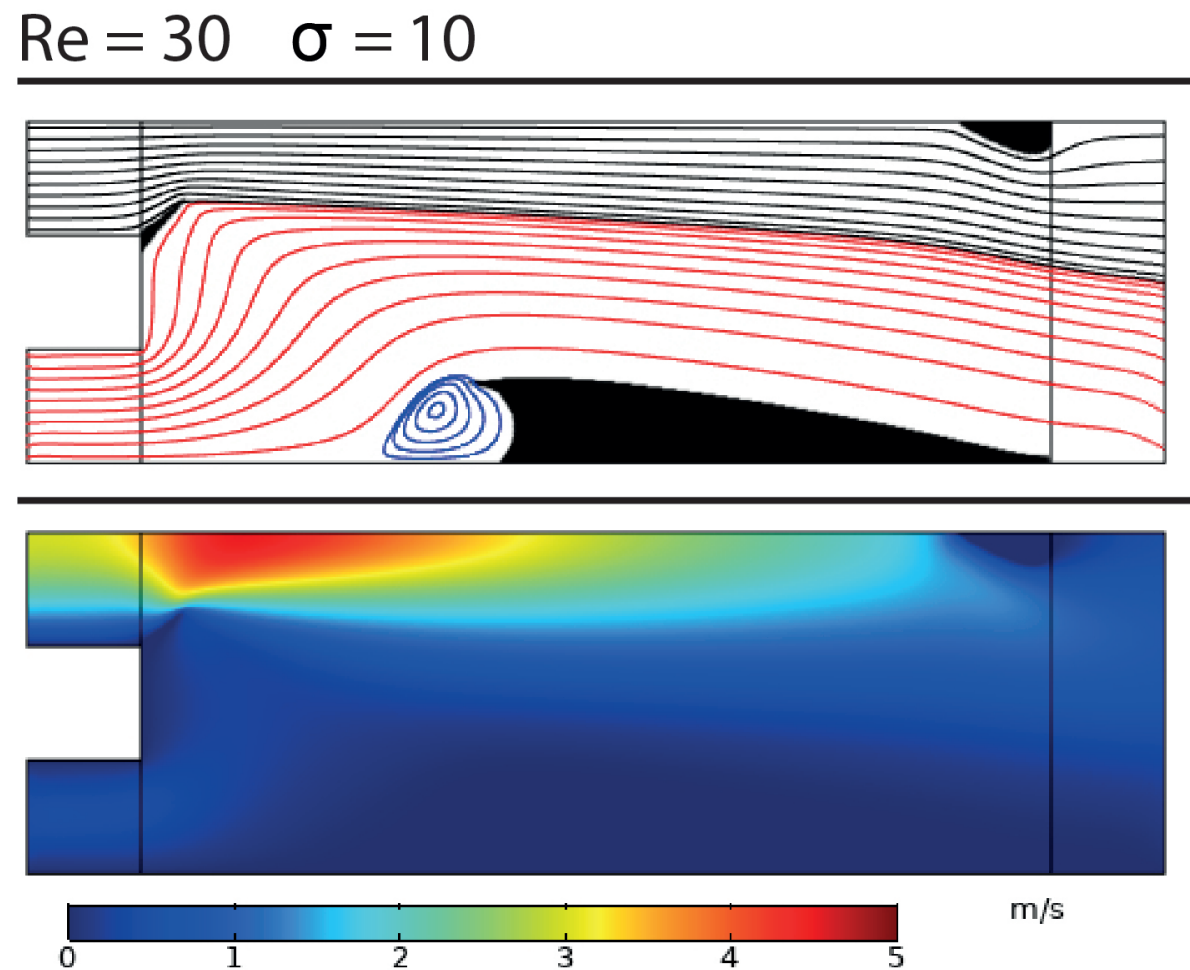

(b)

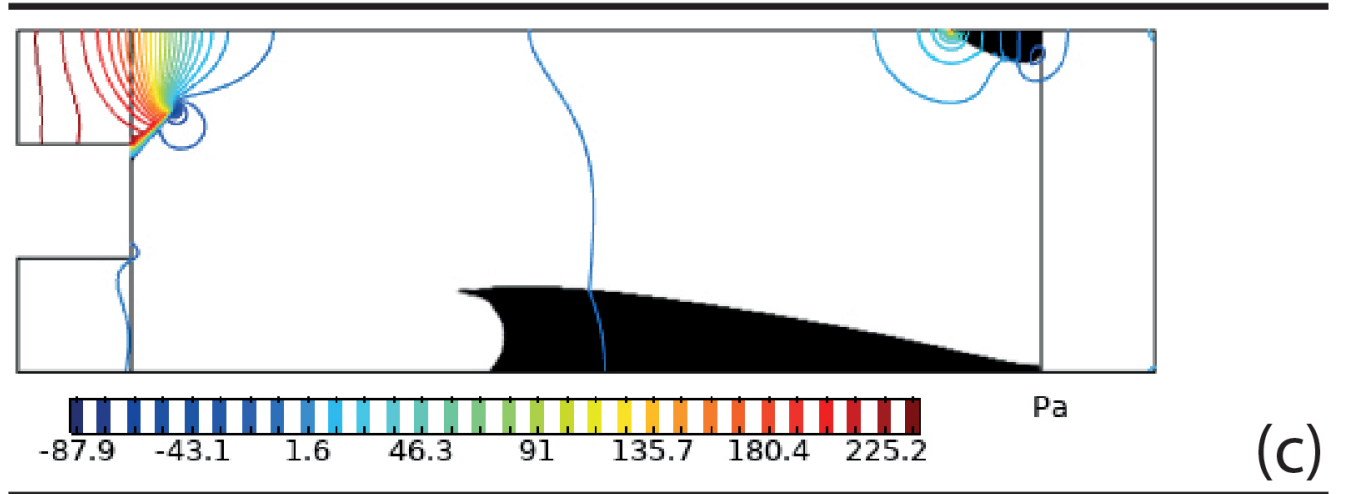

Source: Author. 
Figure 70 - (a) Stream lines seed from the inlet (black) and secondary inlet (red) and recirculation zones (blue) with the solid distribution (black structures), (b) velocity colour map and (c) static pressure contours with the solid distribution (black structures). Results obtained with Reynolds number equal to 30 and $\sigma$ equal to 30 .
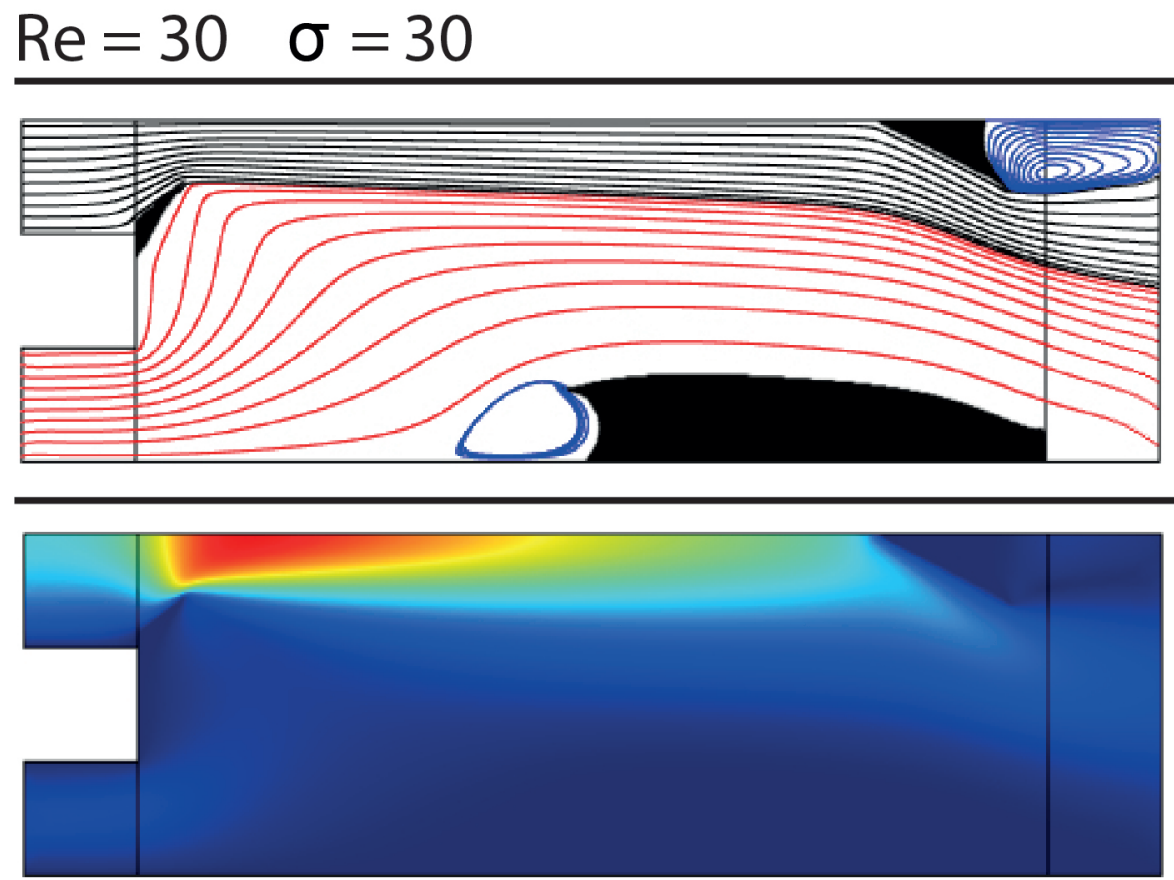

$\mathrm{m} / \mathrm{s}$
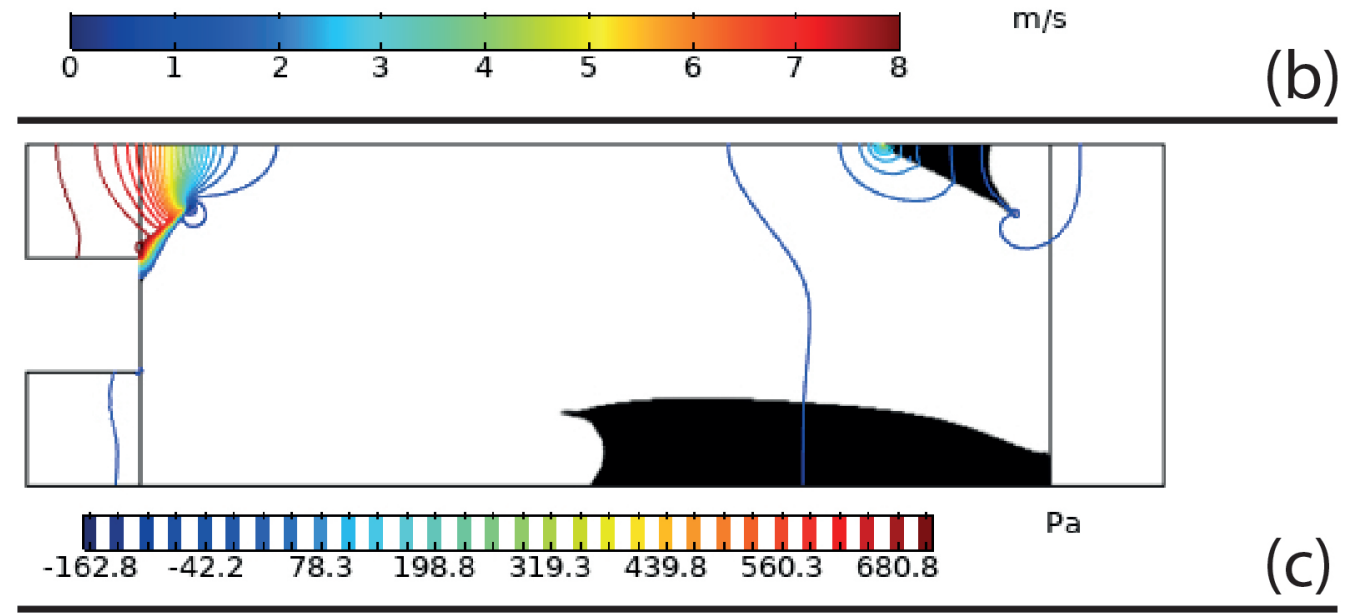

Source: Author. 


\section{APPENDIX C - FLOW FIELD}

For sake of clearance, the plot of the optimized domains using the multi-objective approach were presented in this appendix.

Figure 71 - (a) Stream lines seed from the inlet (black) and secondary inlet (red) with the solid distribution (black structures), (b) velocity colour map and (c) static pressure contours with the solid distribution (black structures). Results obtained with Reynolds number equal to 30, $\sigma$ equal to 30 and $w$ equal to 0.0 .
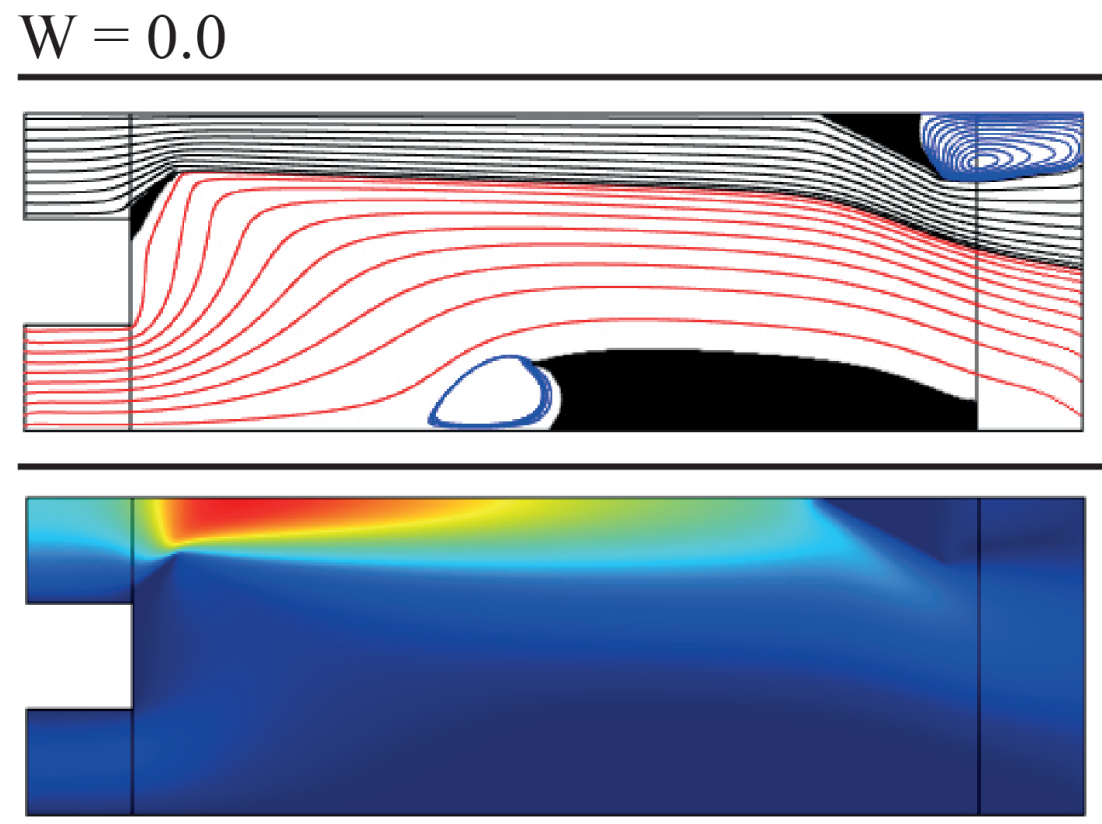

$\mathrm{m} / \mathrm{s}$

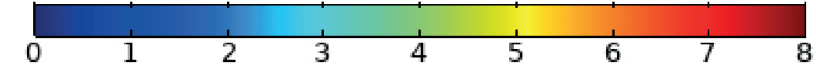

(b)

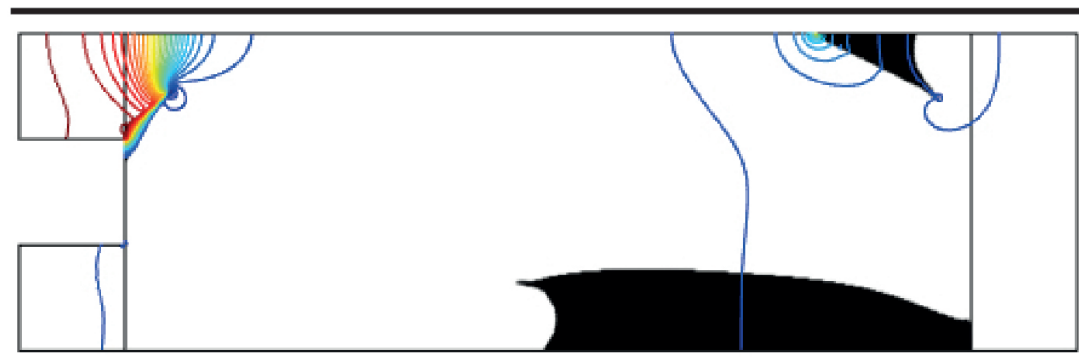

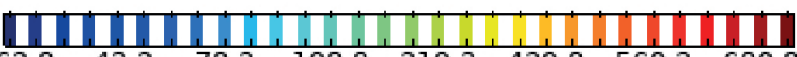

$\mathrm{Pa}$

Source: Author. 
Figure 72 - (a) Stream lines seed from the inlet (black) and secondary inlet (red) with the solid distribution (black structures), (b) velocity colour map and (c) static pressure contours with the solid distribution (black structures). Results obtained with Reynolds number equal to 30, $\sigma$ equal to 30 and $w$ equal to 0.1 .
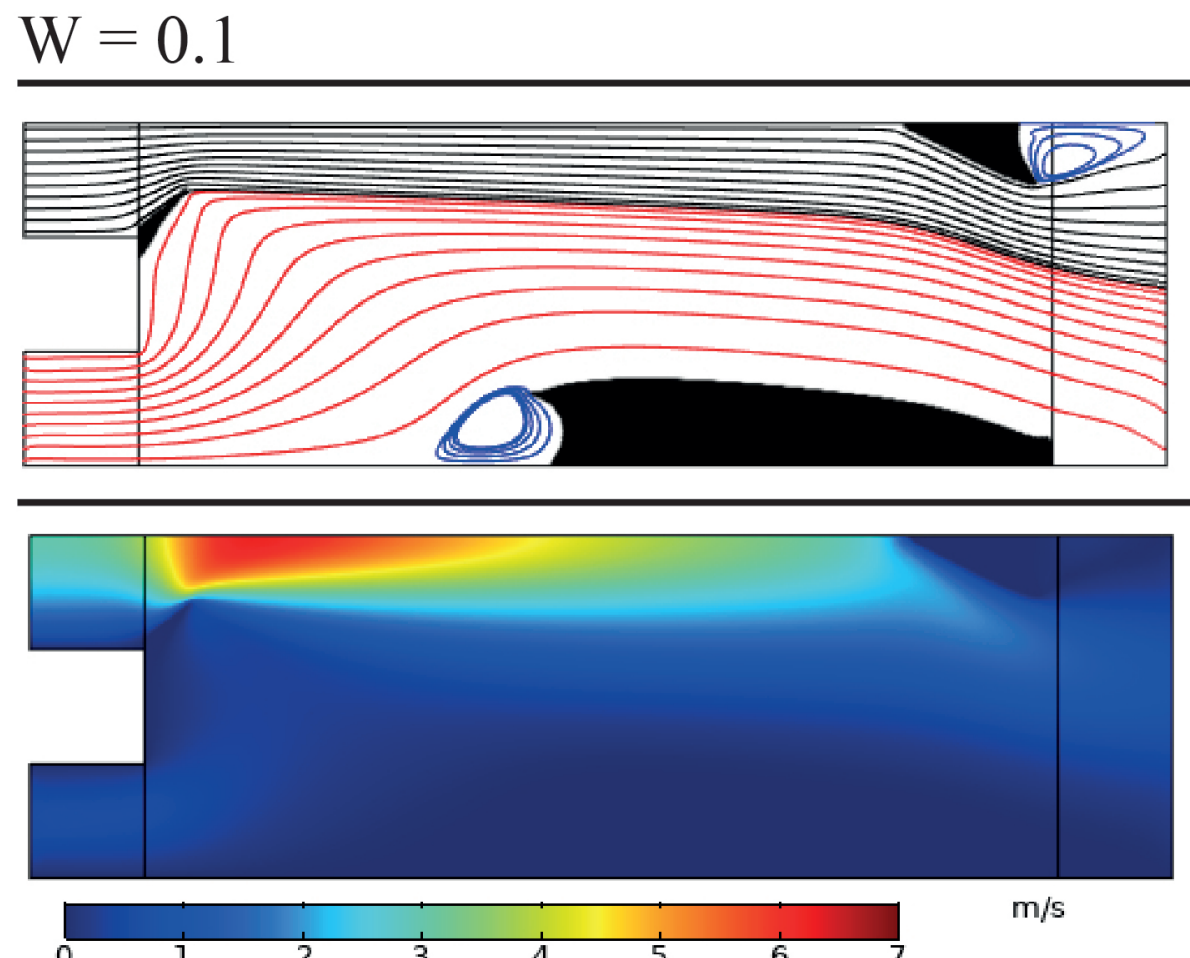

(b)

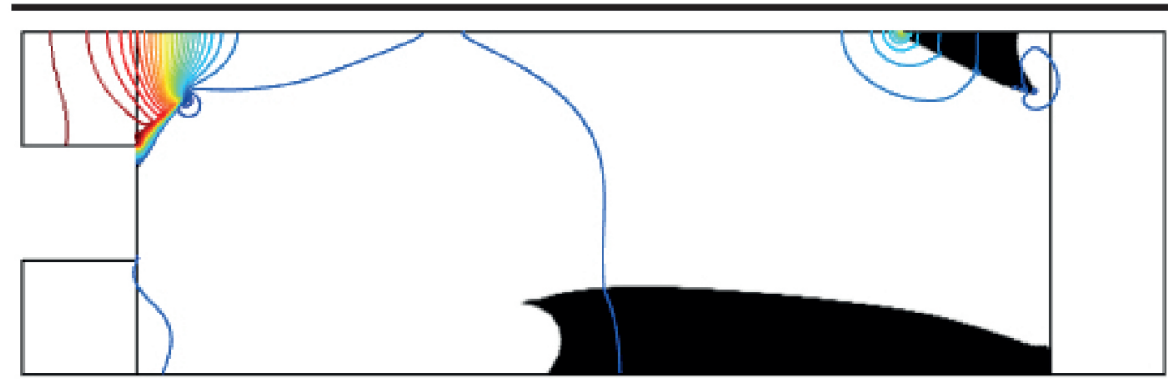

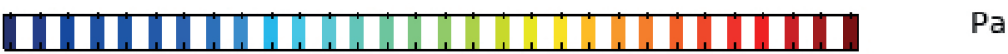

Source: Author. 
Figure 73 - (a) Stream lines seed from the inlet (black) and secondary inlet (red) with the solid distribution (black structures), (b) velocity colour map and (c) static pressure contours with the solid distribution (black structures). Results obtained with Reynolds number equal to 30, $\sigma$ equal to 30 and $w$ equal to 0.2 .

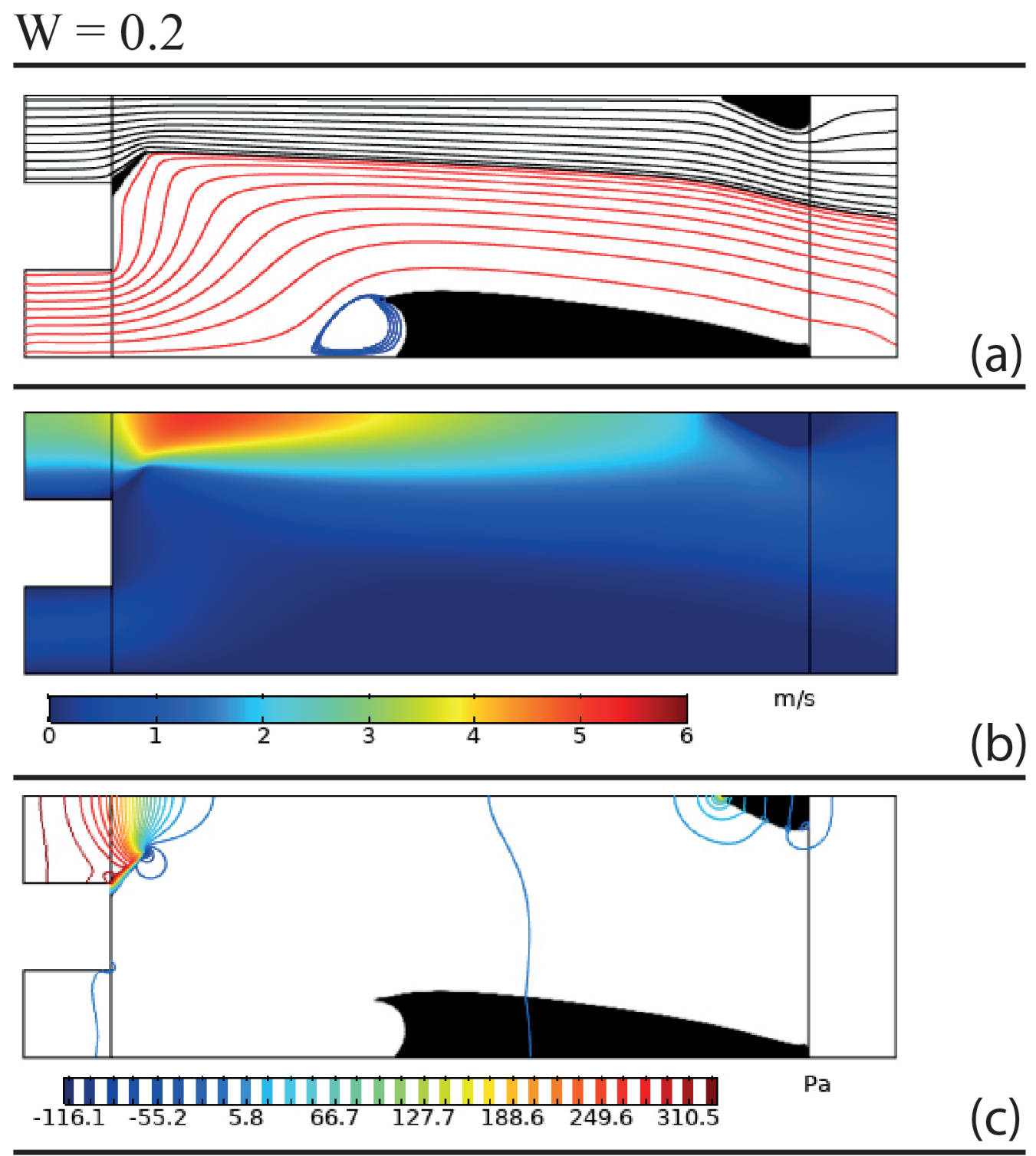

Source: Author. 
Figure 74 - (a) Stream lines seed from the inlet (black) and secondary inlet (red) with the solid distribution (black structures), (b) velocity colour map and (c) static pressure contours with the solid distribution (black structures). Results obtained with Reynolds number equal to 30, $\sigma$ equal to 30 and $w$ equal to 0.3 .

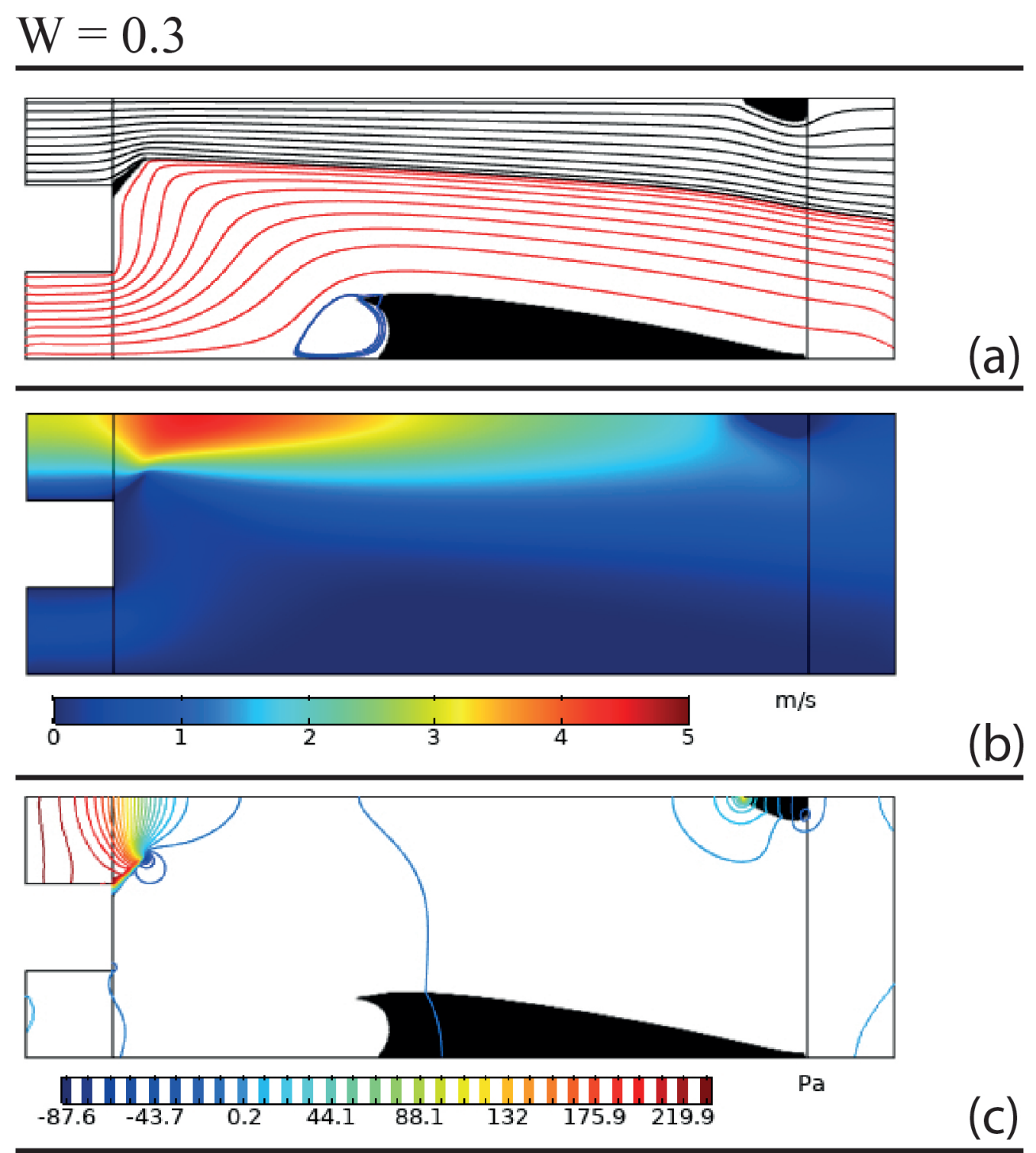

Source: Author. 
Figure 75 - (a) Stream lines seed from the inlet (black) and secondary inlet (red) with the solid distribution (black structures), (b) velocity colour map and (c) static pressure contours with the solid distribution (black structures). Results obtained with Reynolds number equal to 30, $\sigma$ equal to 30 and $w$ equal to 0.4 .

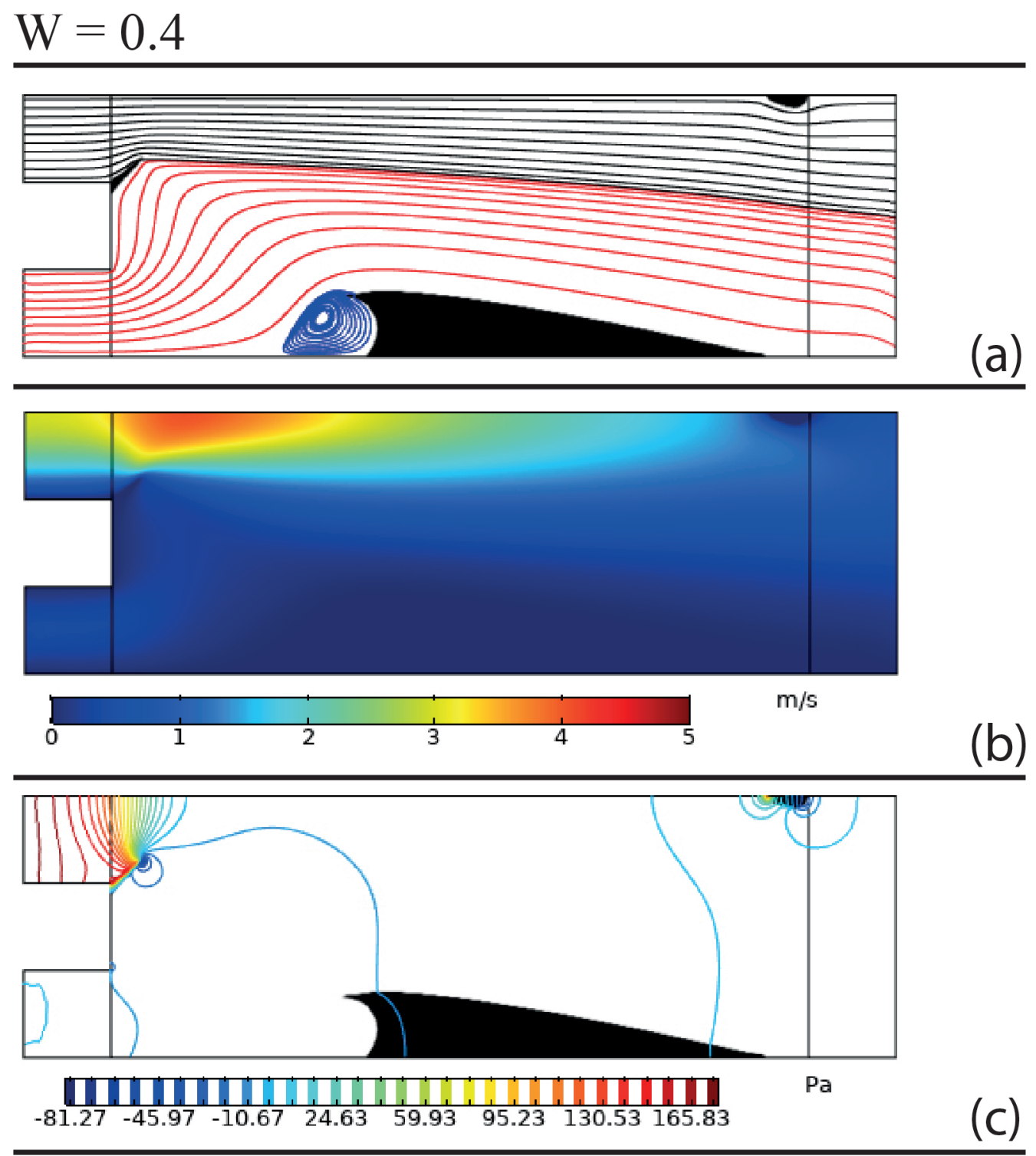

Source: Author. 
Figure 76 - (a) Stream lines seed from the inlet (black) and secondary inlet (red) with the solid distribution (black structures), (b) velocity colour map and (c) static pressure contours with the solid distribution (black structures). Results obtained with Reynolds number equal to 30, $\sigma$ equal to 30 and $w$ equal to 0.5 .

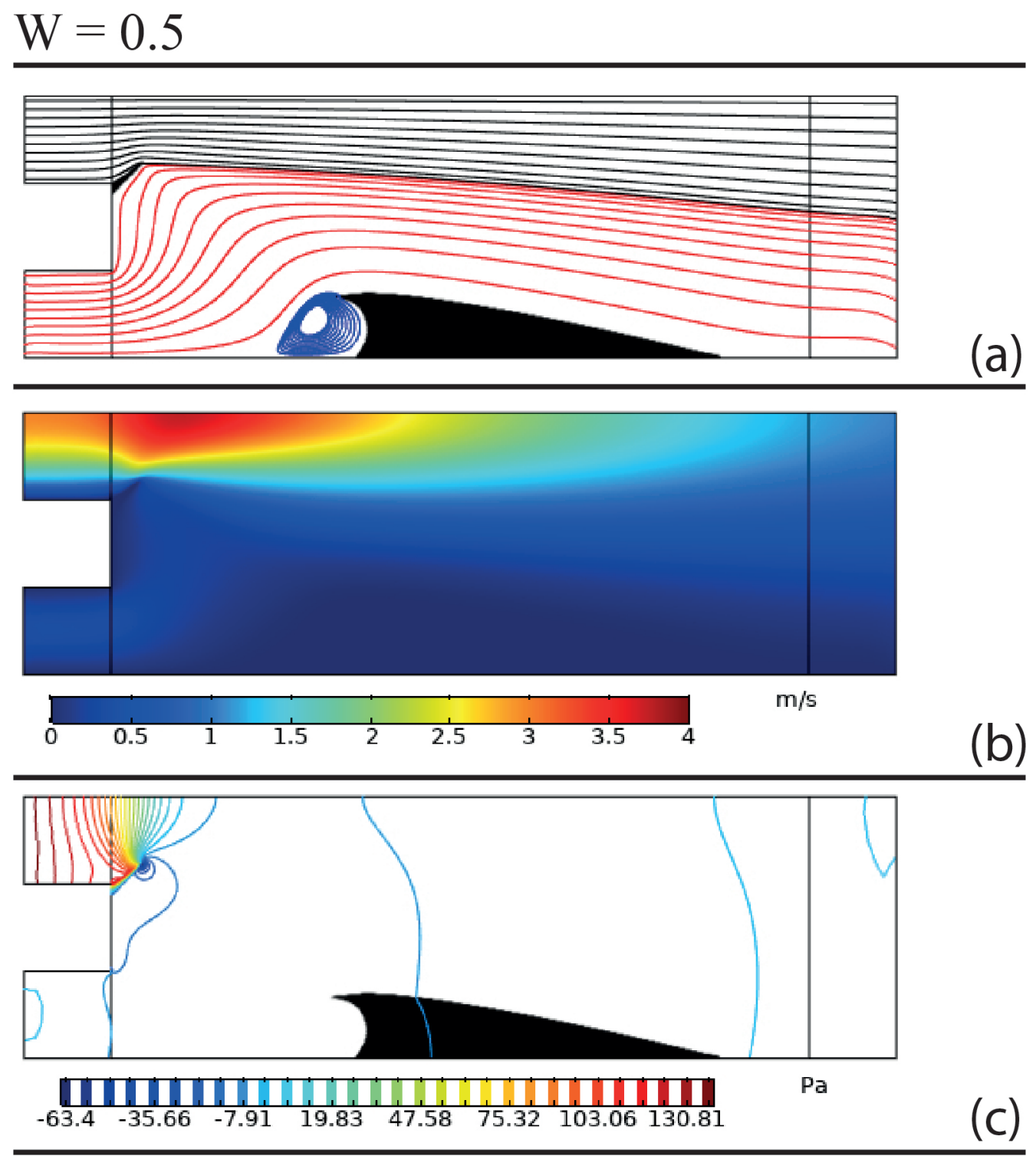

Source: Author. 
Figure 77 - (a) Stream lines seed from the inlet (black) and secondary inlet (red) with the solid distribution (black structures), (b) velocity colour map and (c) static pressure contours with the solid distribution (black structures). Results obtained with Reynolds number equal to 30, $\sigma$ equal to 30 and $w$ equal to 0.6 .

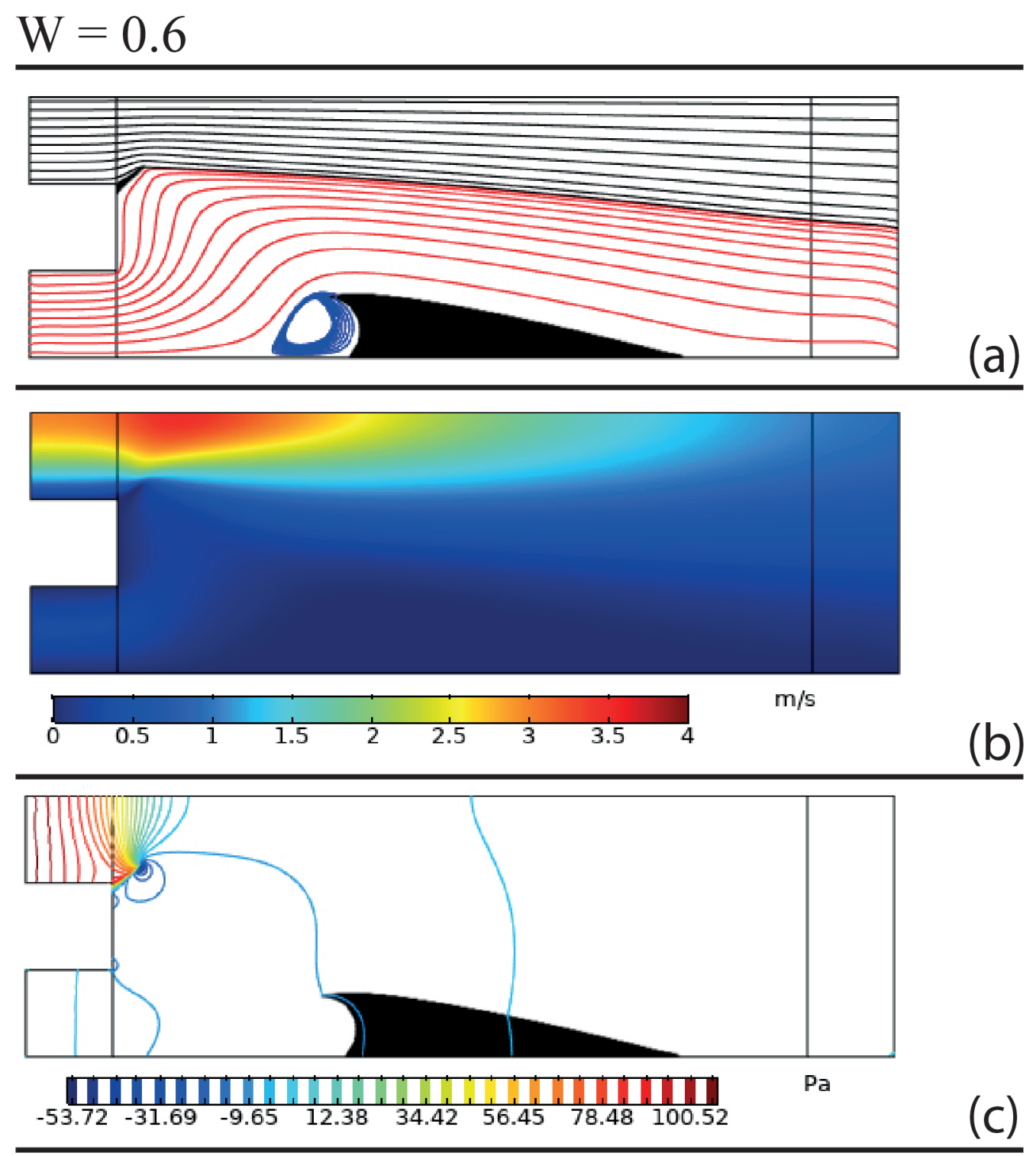

Source: Author. 
Figure 78 - (a) Stream lines seed from the inlet (black) and secondary inlet (red) with the solid distribution (black structures), (b) velocity colour map and (c) static pressure contours with the solid distribution (black structures). Results obtained with Reynolds number equal to 30, $\sigma$ equal to 30 and $w$ equal to 0.7 .

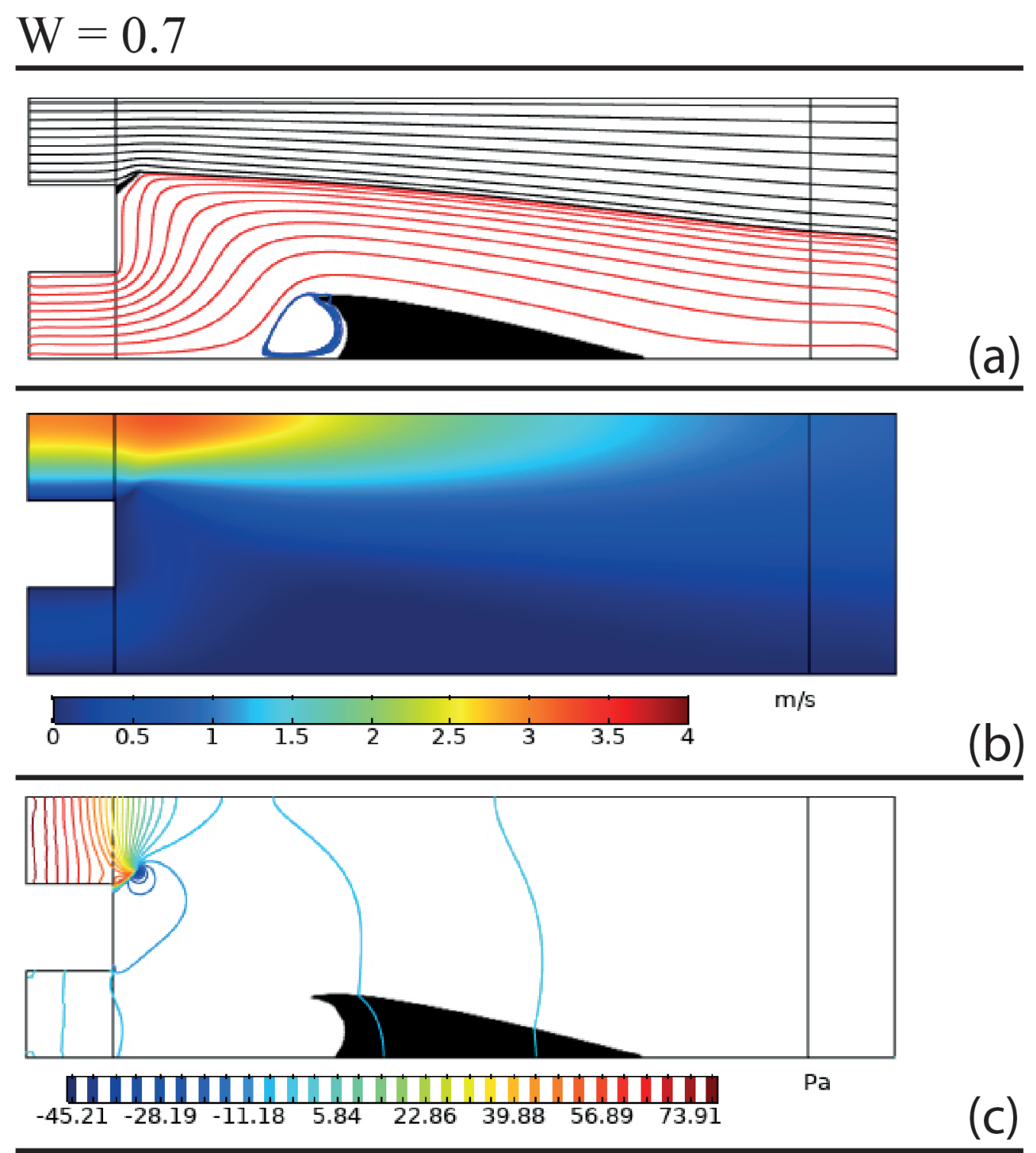

Source: Author. 
Figure 79 - (a) Stream lines seed from the inlet (black) and secondary inlet (red) with the solid distribution (black structures), (b) velocity colour map and (c) static pressure contours with the solid distribution (black structures). Results obtained with Reynolds number equal to 30, $\sigma$ equal to 30 and $w$ equal to 0.8 .

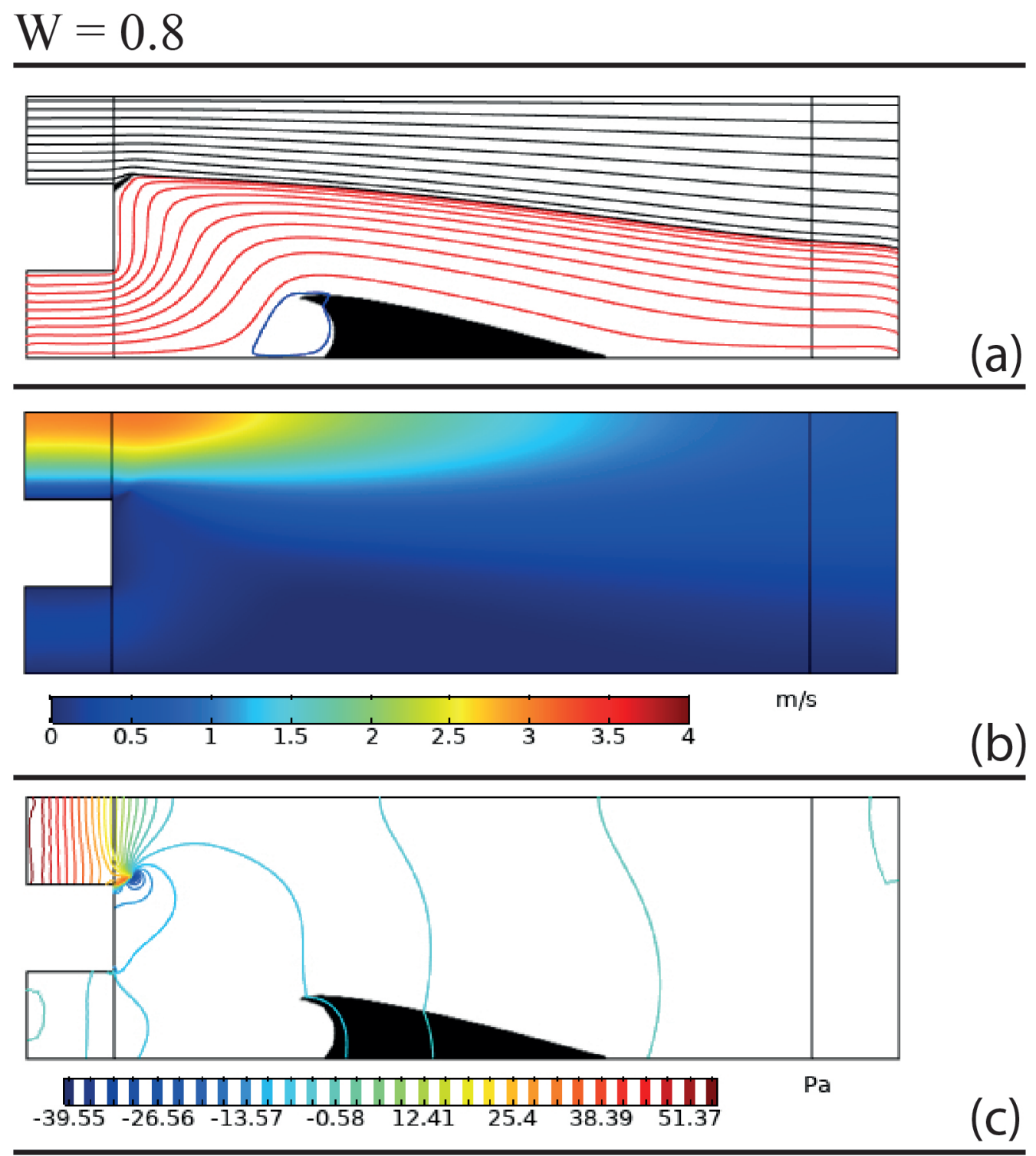

Source: Author. 
Figure 80 - (a) Stream lines seed from the inlet (black) and secondary inlet (red) with the solid distribution (black structures), (b) velocity colour map and (c) static pressure contours with the solid distribution (black structures). Results obtained with Reynolds number equal to 30, $\sigma$ equal to 30 and $w$ equal to 0.9 .

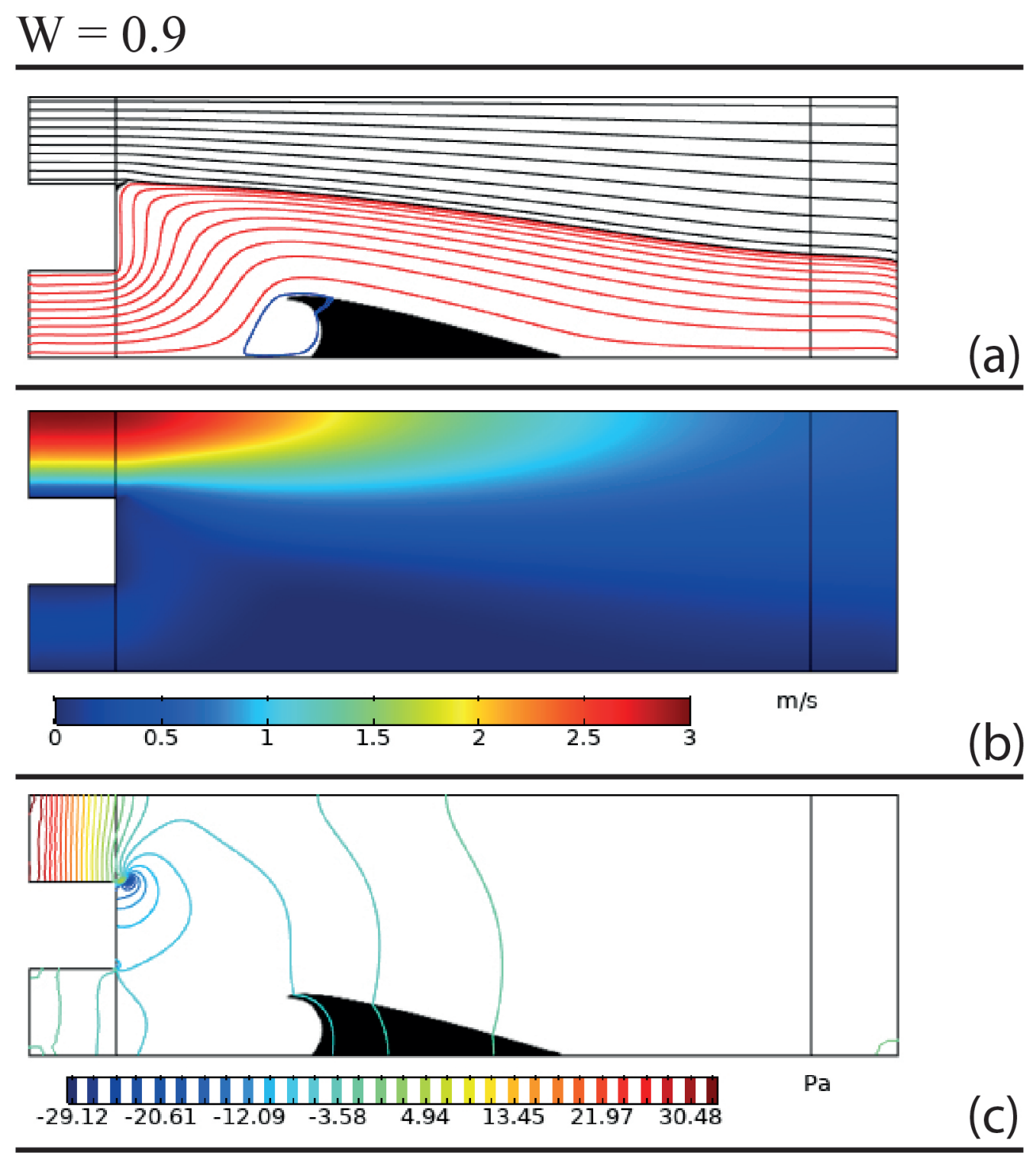

Source: Author. 
Figure 81 - (a) Stream lines seed from the inlet (black) and secondary inlet (red) with the solid distribution (black structures), (b) velocity colour map and (c) static pressure contours with the solid distribution (black structures). Results obtained with Reynolds number equal to 30, $\sigma$ equal to 30 and $w$ equal to 0.925 .

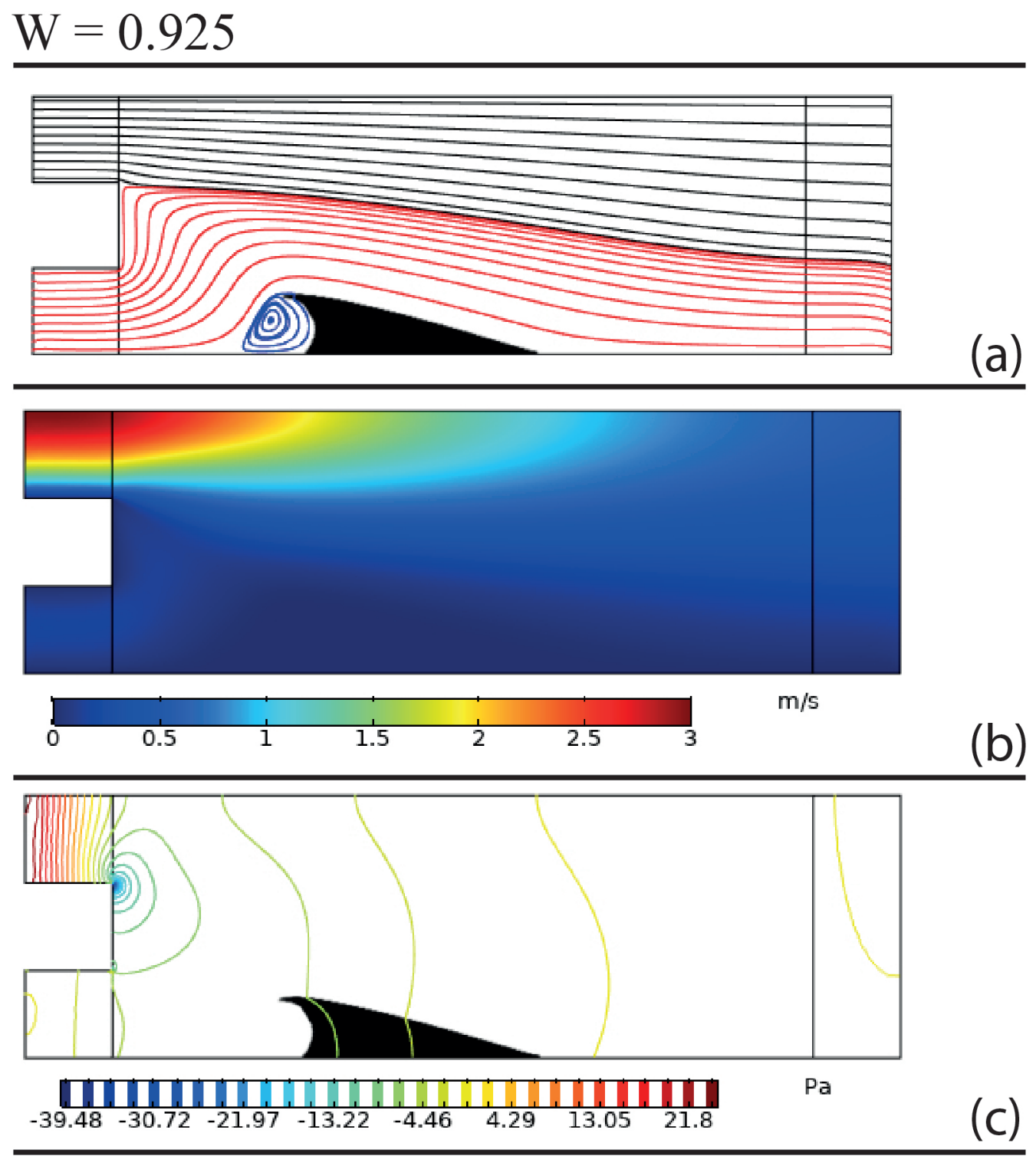

Source: Author. 
Figure 82 - (a) Stream lines seed from the inlet (black) and secondary inlet (red) with the solid distribution (black structures), (b) velocity colour map and (c) static pressure contours with the solid distribution (black structures). Results obtained with Reynolds number equal to 30, $\sigma$ equal to 30 and $w$ equal to 0.95 .

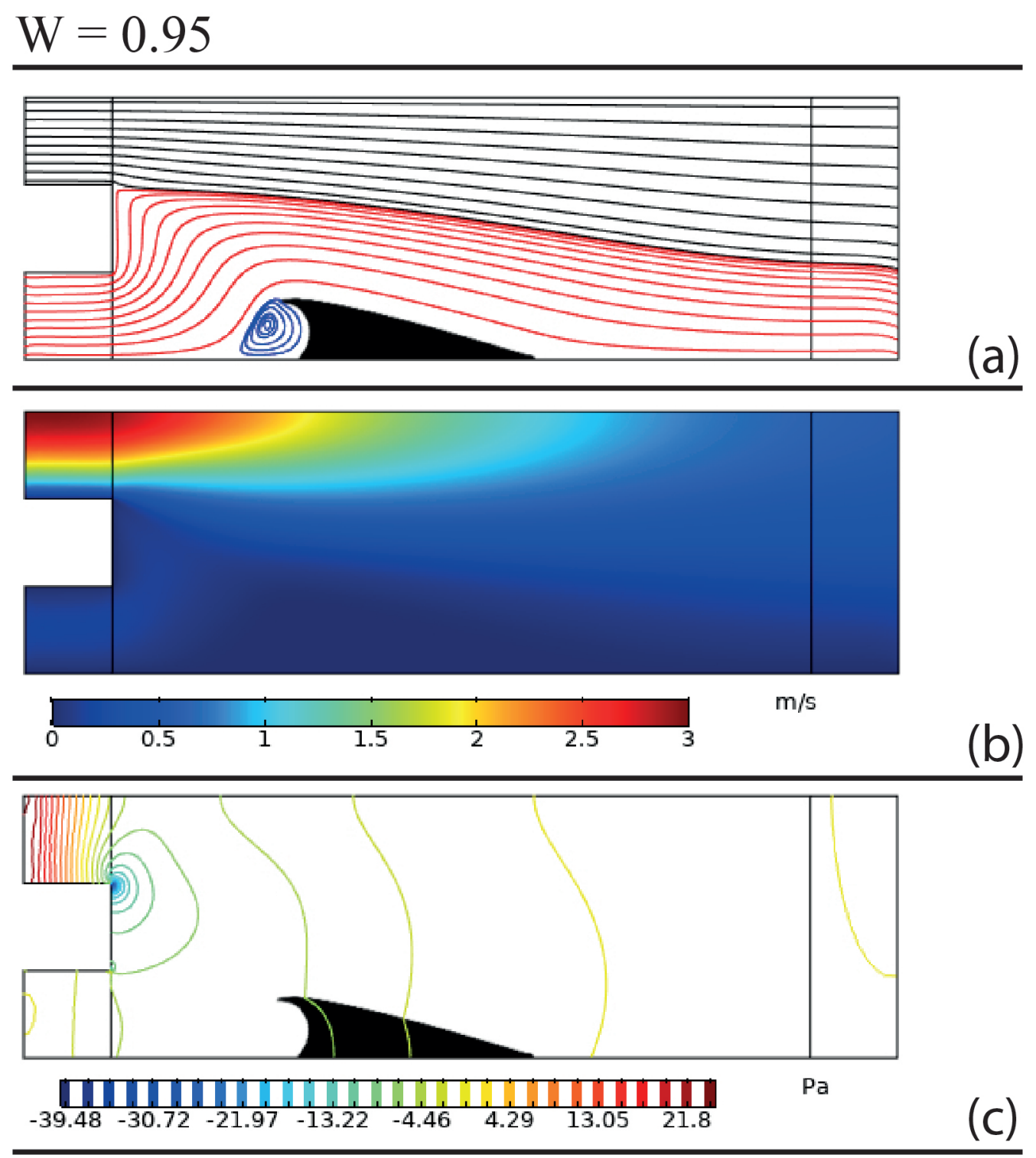

Source: Author. 
Figure 83 - (a) Stream lines seed from the inlet (black) and secondary inlet (red) with the solid distribution (black structures), (b) velocity colour map and (c) static pressure contours with the solid distribution (black structures). Results obtained with Reynolds number equal to 30, $\sigma$ equal to 30 and $w$ equal to 0.975 .

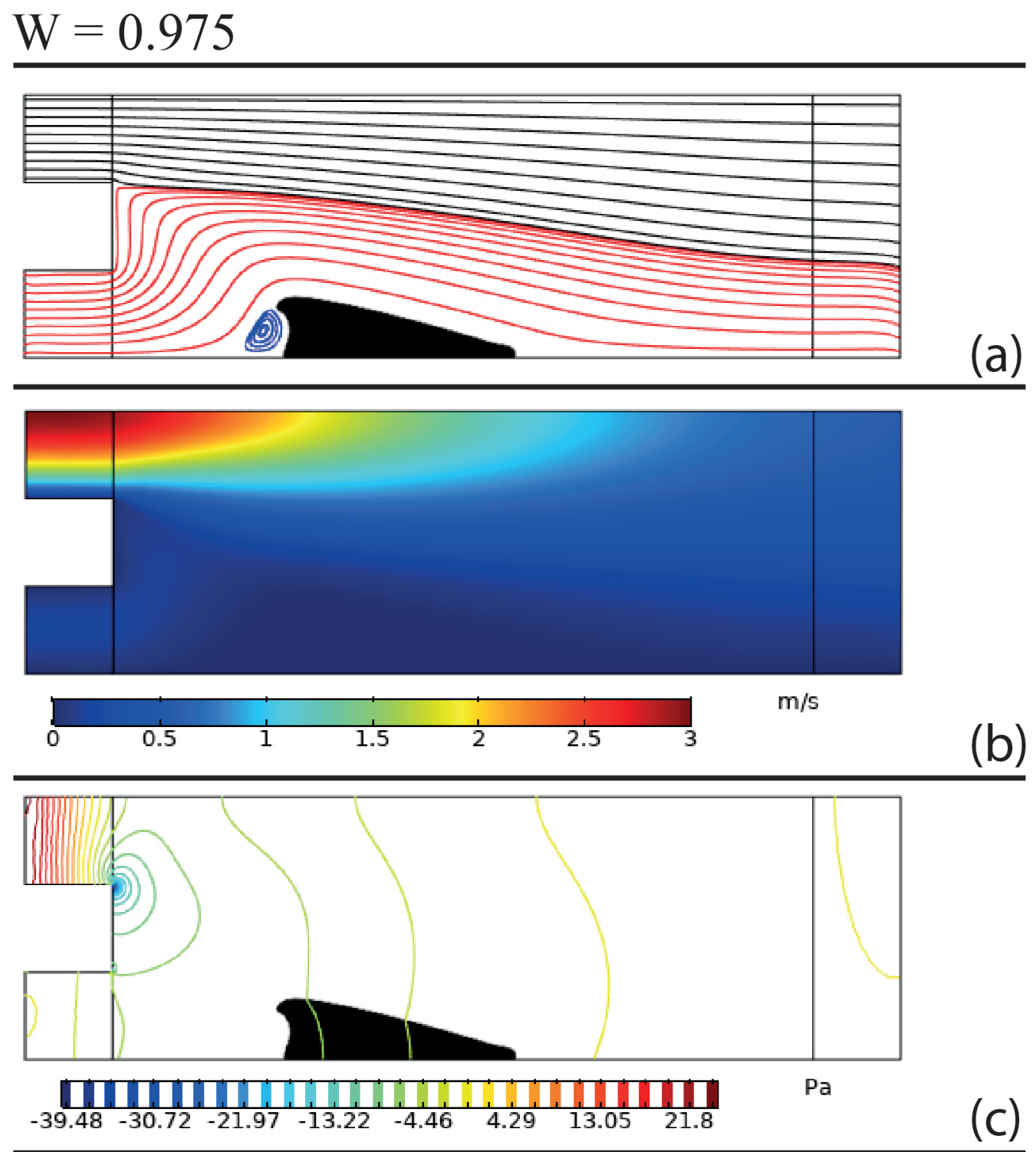

Source: Author. 
Figure 84 - (a) Stream lines seed from the inlet (black) and secondary inlet (red) with the solid distribution (black structures), (b) velocity colour map and (c) static pressure contours with the solid distribution (black structures). Results obtained with Reynolds number equal to 30, $\sigma$ equal to 30 and $w$ equal to 0.99 .

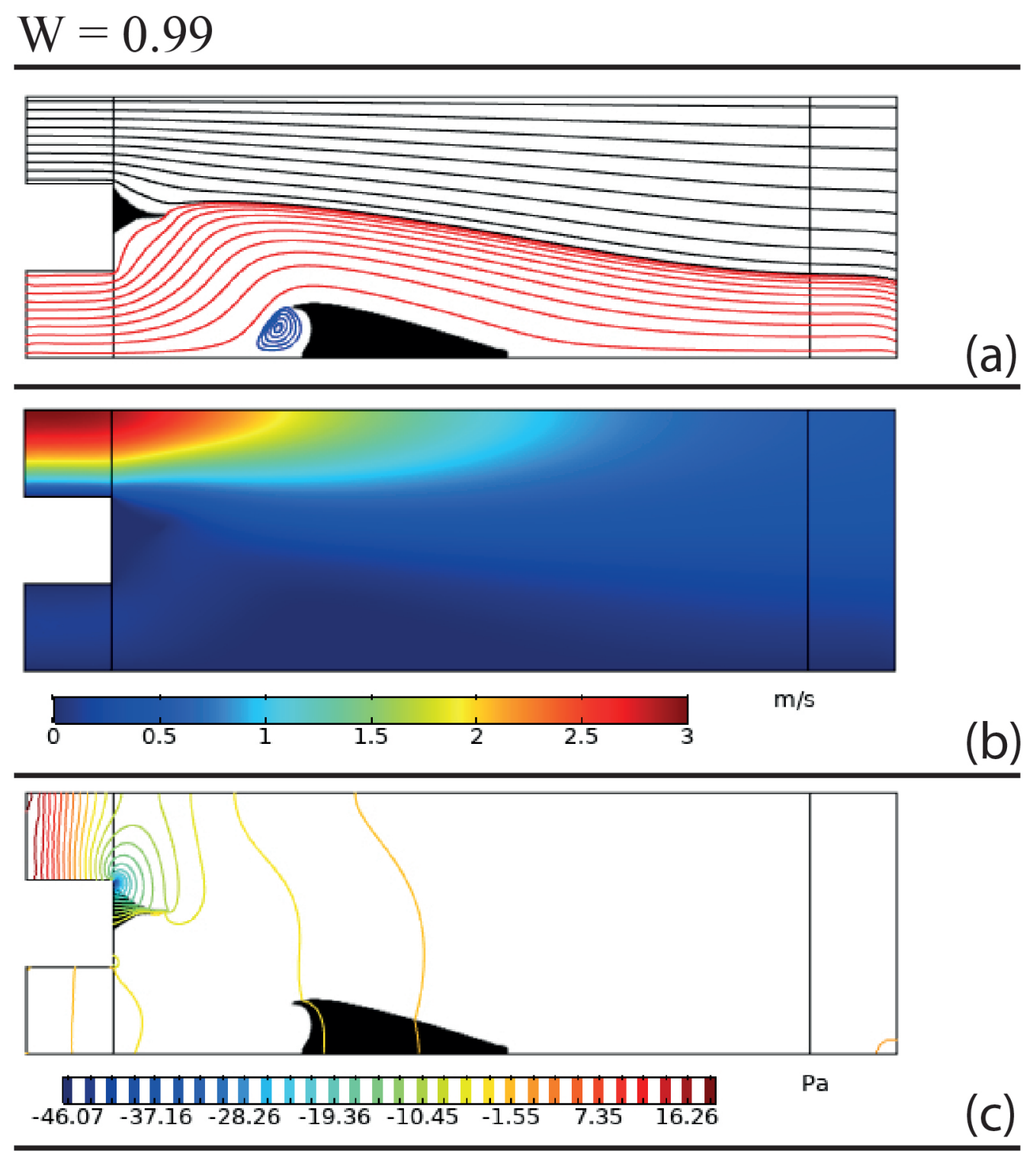

Source: Author. 
Figure 85 - (a) Stream lines seed from the inlet (black) and secondary inlet (red) with the solid distribution (black structures), (b) velocity colour map and (c) static pressure contours with the solid distribution (black structures). Results obtained with Reynolds number equal to 30, $\sigma$ equal to 30 and $w$ equal to 0.995 .

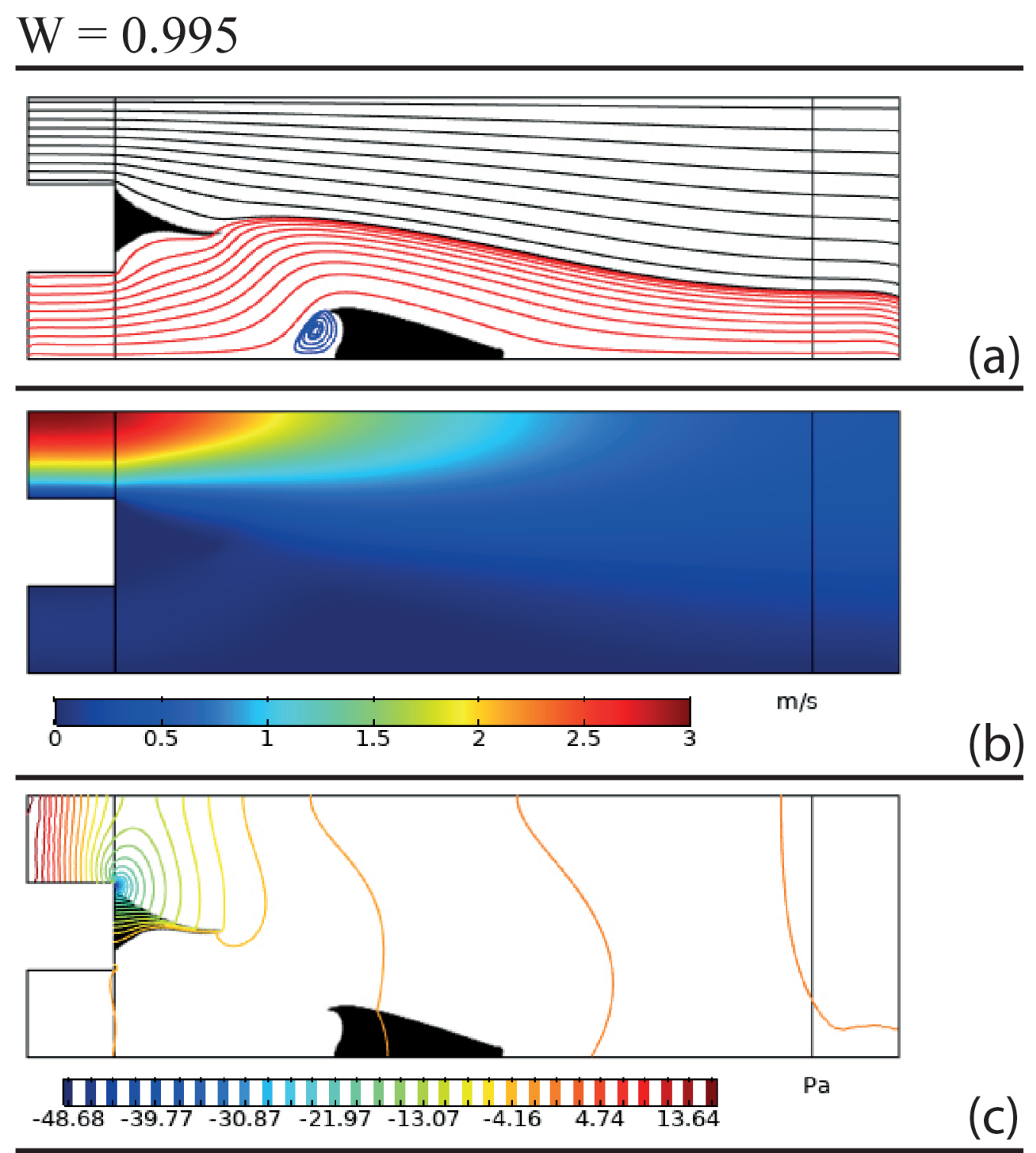

Source: Author. 
Figure 86 - (a) Stream lines seed from the inlet (black) and secondary inlet (red) with the solid distribution (black structures), (b) velocity colour map and (c) static pressure contours with the solid distribution (black structures). Results obtained with Reynolds number equal to 30, $\sigma$ equal to 30 and $w$ equal to 1.0.

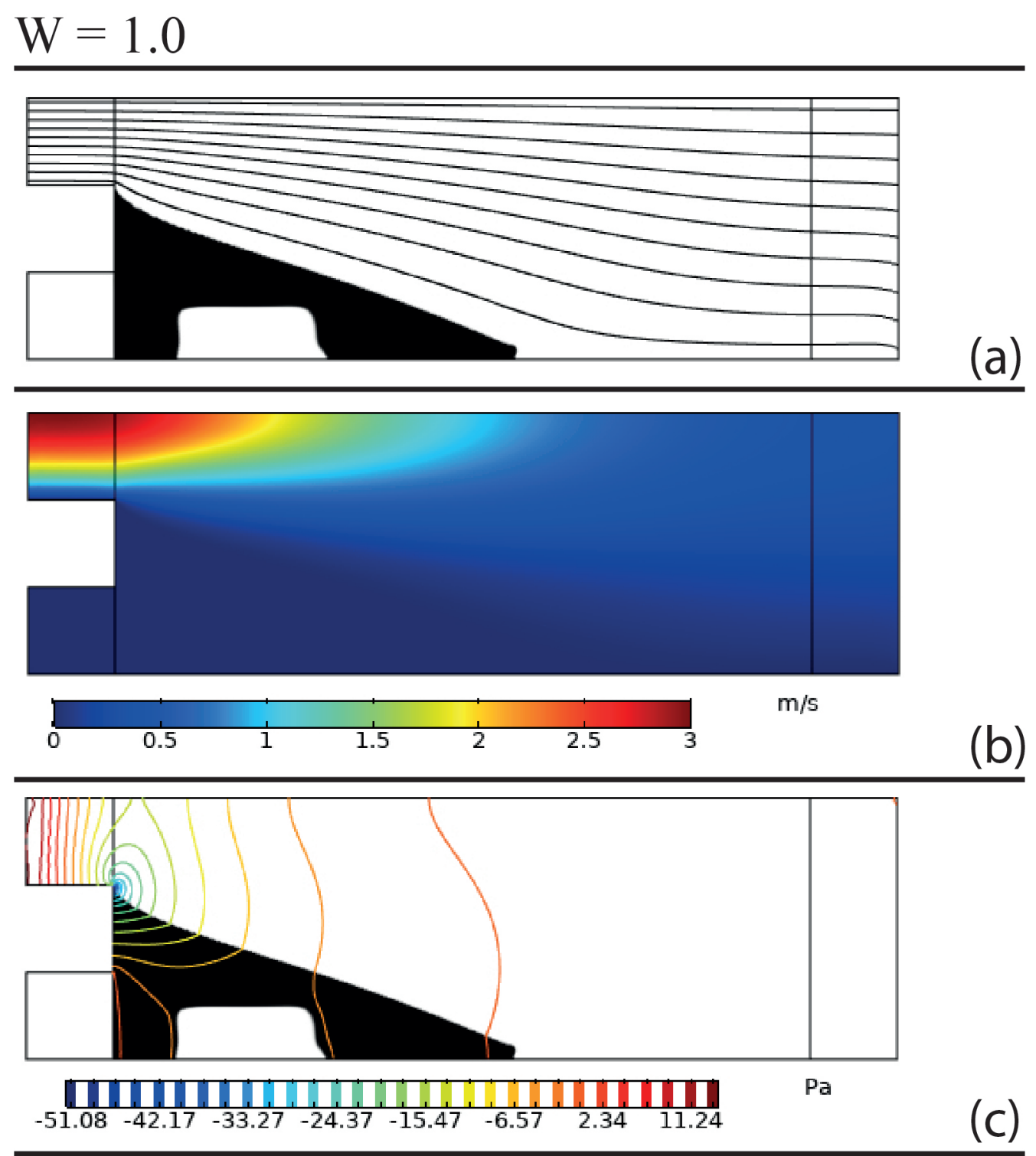

Source: Author. 


\section{APPENDIX D - MESH CONVERGENCE}

The geometries 1, 2 and 3 were simulated varying the ratio in equation D.1

$$
h_{m s h}=\frac{H}{\text { ratio }}
$$

Where $h_{m s h}$ is he maximum element size and ratio is a real number. To evaluate the flow modification due to the mesh refinement, the integral of the velocity magnitude $U_{m}$ over the entire domain $\Omega_{a}$ was evaluated for each proposed ratio. This quantity received the symbol $M_{u}$

$$
M_{u}=\int_{\Omega_{a}} U_{m} d \Omega_{a}
$$

The result of the sweep over ratio values is presented in Figure 87.

Figure 87 - Plot of quantity $M_{u}$ in function of ratio values for geometry 1 (black), geometry 2 (green) and geometry 3 (blue). The dashed indicates the ratio of 10 used in the course mesh.

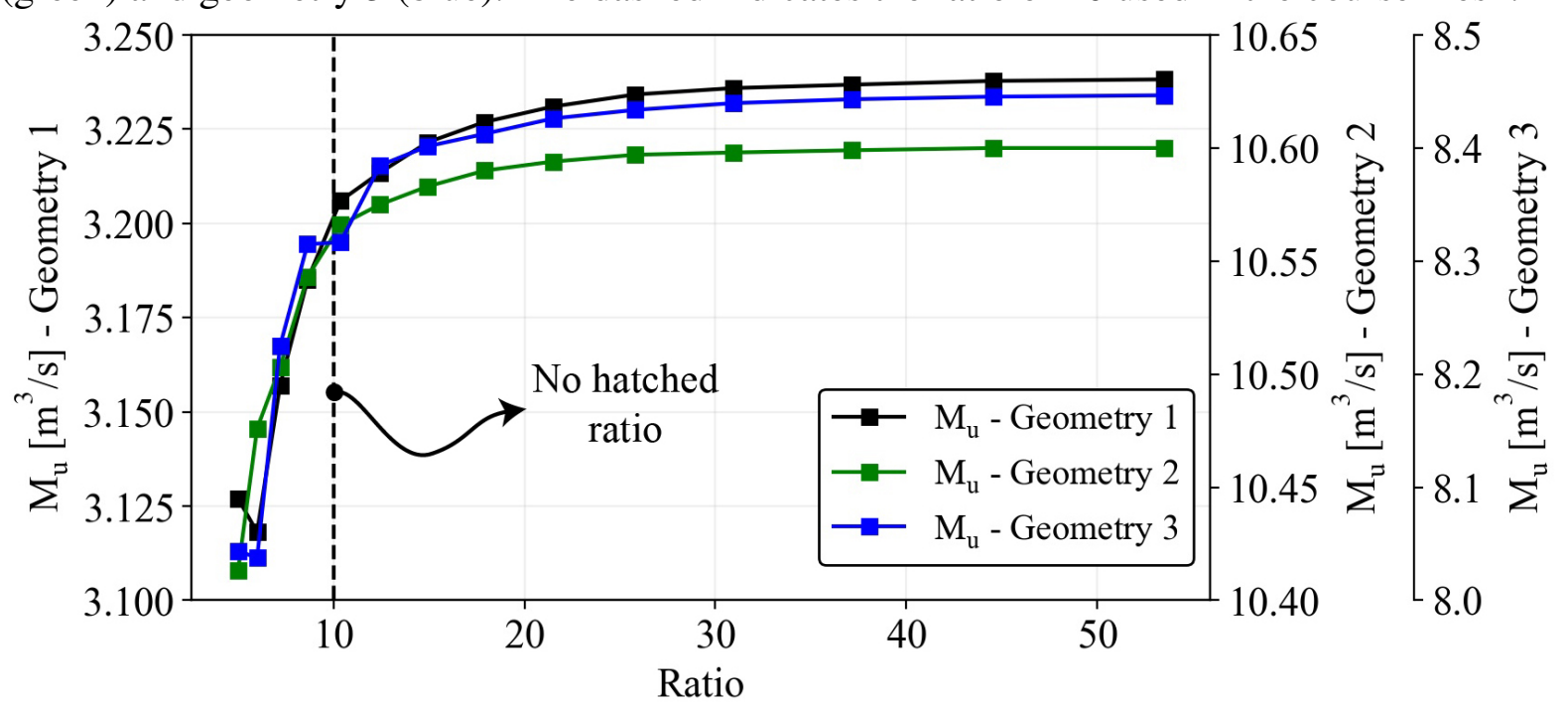

Source: Author.

As it is possible to observe, converge happens, for all geometries, with ratio about 30. However, due to topology optimization, the mesh inside the hatched region must be increased 
to a ratio equal to 100 , drastically increasing the computational cost. To reduce the simulation time, the mesh in the no hatched area was coursed below the ratio equal to 30 . This approach certainly reduces the accuracy of the flow in these areas and the value of objective function measured at outlet and secondary inlet. Nonetheless, the result of topology optimization was not effected. As depicted in Figure 88 to 90 the plot of external contours to the fine and coursed mesh are the same.

Figure 88 - Plot of external contours of solid distribution taken from results obtained with the fine mesh and course mesh for geometry 1 . The Reynolds number was set up equal to 20 and $\sigma$ equal to 10 .

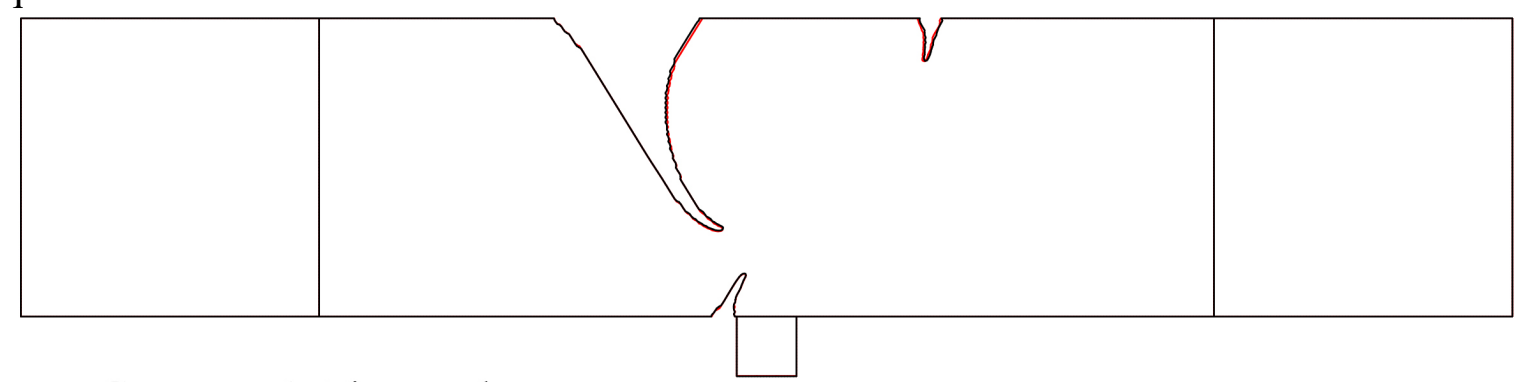

Geometry 1- Fine mesh

$\square$ Geometry 1 - Course mesh

Figure 89 - Plot of external contours of solid distribution taken from results obtained with the fine mesh and course mesh for geometry 2. The Reynolds number was set up equal to 30 and $\sigma$ equal to 30 .

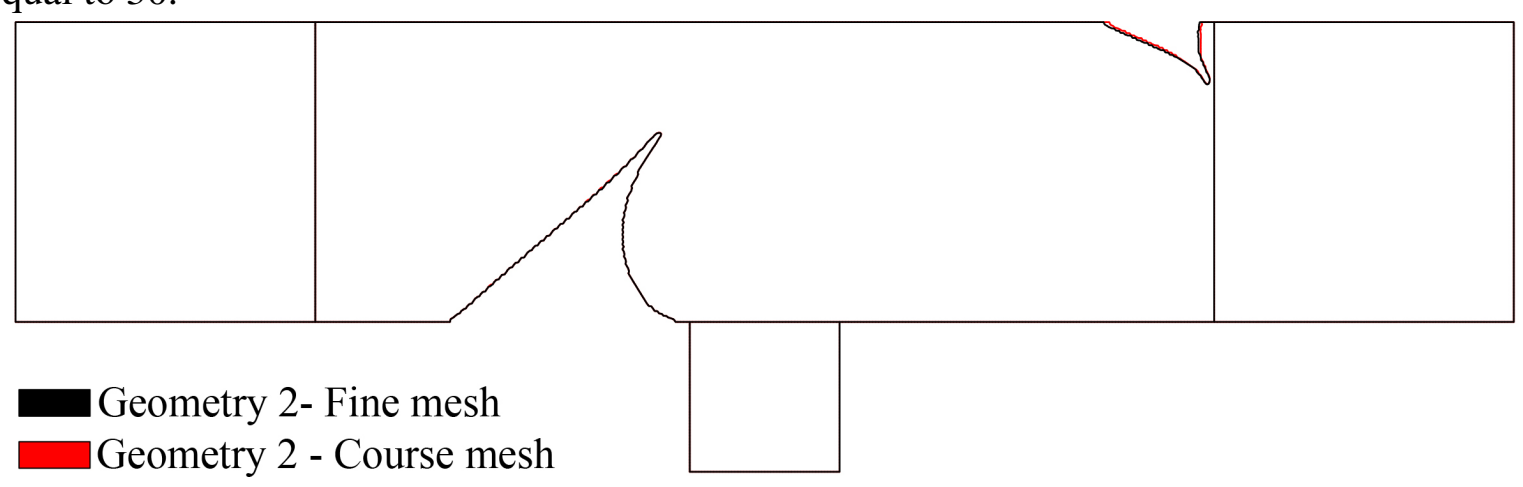

The importance of such approach with respect to the mesh can be visualized in table 10 that compares the time for simulating with the fine and course meshes. 
Figure 90 - Plot of external contours of solid distribution taken from results obtained with the fine mesh and course mesh for geometry 3. The Reynolds number was set up equal to 30 and $\sigma$ equal to 30 .

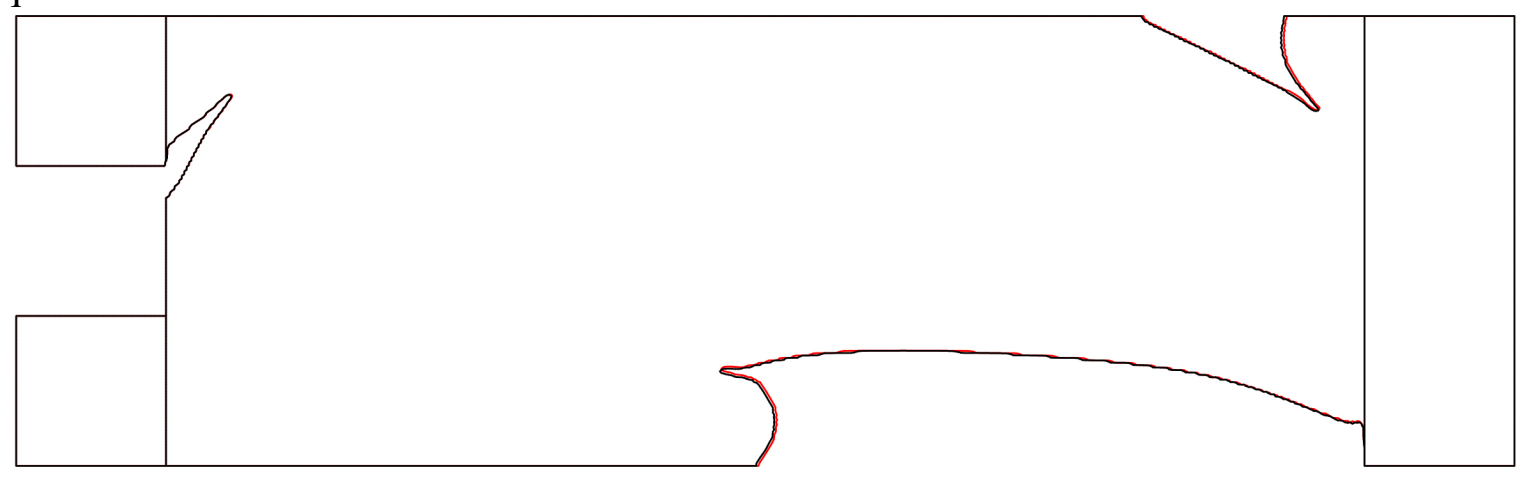

Geometry 2- Fine mesh

$\square$ Geometry 2 - Course mesh

Table 10 - Table of relative difference between two consecutive $N$ values. All used data are in percentage.

\begin{tabular}{ccc}
\hline Geometry & Fine Mesh & Course mesh \\
\hline 1 & $27 \mathrm{~h} 11 \mathrm{~min}$ & $13 \mathrm{~h} 37 \mathrm{~min}$ \\
\hline 2 & $36 \mathrm{~h} 4 \mathrm{~min}$ & $20 \mathrm{~h} 36 \mathrm{~min}$ \\
\hline 3 & $66 \mathrm{~h} 58 \mathrm{~min}$ & $59 \mathrm{~h} 27 \mathrm{~min}$ \\
\hline
\end{tabular}

Source: Author. 


\section{APPENDIX E - RECIRCULATION STRUCTURES AT OUTLET}

The simulations results for Reynolds number equal to 30 and parameter $\sigma$ equal 30 for RSI and Reynolds number equal to 30 and parameter $\sigma$ equal to 10 and 30 for ASI, shown recirculation structures at the outlet boundary region. Due to the boundary conditions set up to this region that is given in function of the normal velocity gradient (equations E.1 to E.3), it was given rise to the question of how much the recirculation behaviour affects the topology optimization result.

- Secondary inlet:

$$
\oint_{\Gamma_{S}}\left[-p+2 \mu\left(\frac{\partial u_{z, r}}{\partial x_{z, r}}\right)\right] w_{z, r} d \Gamma=0
$$

- Outlet:

$$
\oint_{\Gamma_{o}}\left[-p+2 \mu\left(\frac{\partial u_{z}}{\partial x_{z}}\right)\right] w_{z} d \Gamma=0
$$

With the imposition of this kind of boundary conditions, the pressure, at the same boundary, is implicitly constrained by

$$
\overbrace{p=2 \mu \frac{\partial u_{z}}{\partial x_{z}}}^{\text {at } \Gamma_{\mathrm{o}}} \text { and } \overbrace{p=2 \mu \frac{\partial u_{z, r}}{\partial x_{z, r}}}^{\text {at }}
$$

Where, for secondary inlet, $z$ coordinate was used for ASI and $r$ for the RSI. To answer the question, another simulation was conducted with ASI case, Reynolds number and parameter $\sigma$ equal to 30 (where the recirculation is more prominent) was chosen. The simulation had an outlet extension 4 times larger than the original one, the same strategy of mesh density that the original case and a proper reference static pressure for the constraint (once the geometry changes). All other simulations set up were the same that in original geometry simulation. 
Figure 91 shows the filtered contours of the solid regions. In this, it is possible to observe a significant difference in the structures. There, the upper structure decreases and the bottom increases around the outlet region of the design domain when compered with the original geometry 3. Hence, differences in the velocity (Figure 92), static pressure (Figure 93) fields and streamlines (Figure 94) were observed. Despite of these differences, the solution found by the optimization algorithm shown similarities with the solution found in the original case, specifically in the flow acceleration near the outlet region to drop the static pressure inside the design domain, thus, enhancing the objective function $J_{2}$. Therefore, because of the similarity involving the physics of the solution and the increase in simulation time (about 55 hours in original geometry and 72 hours to extended), the original domain was used.

Figure 91 - Comparison between the solid and void distribution of the original and extended geometries.

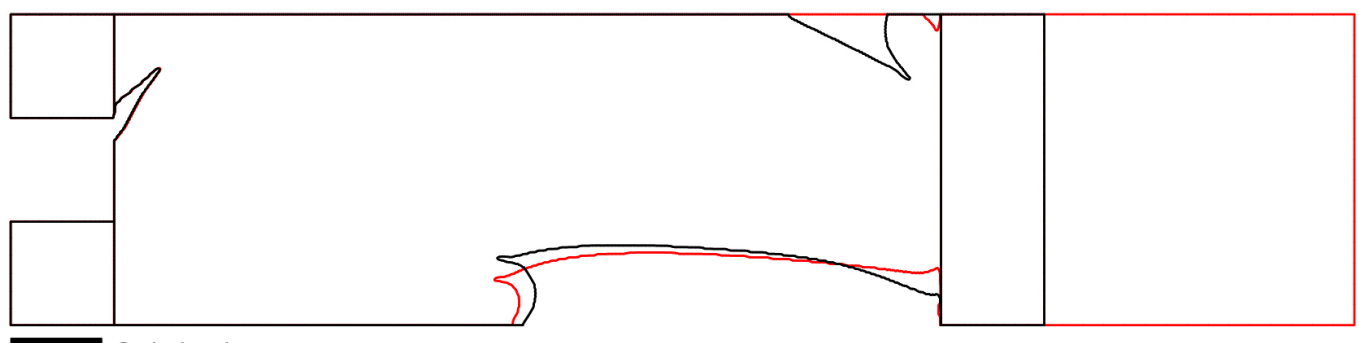

Original geometry 3

$\square$ Extended geometry 3

Source: Author.

Figure 92 - Comparison between the velocity field of the original and extended geometries.

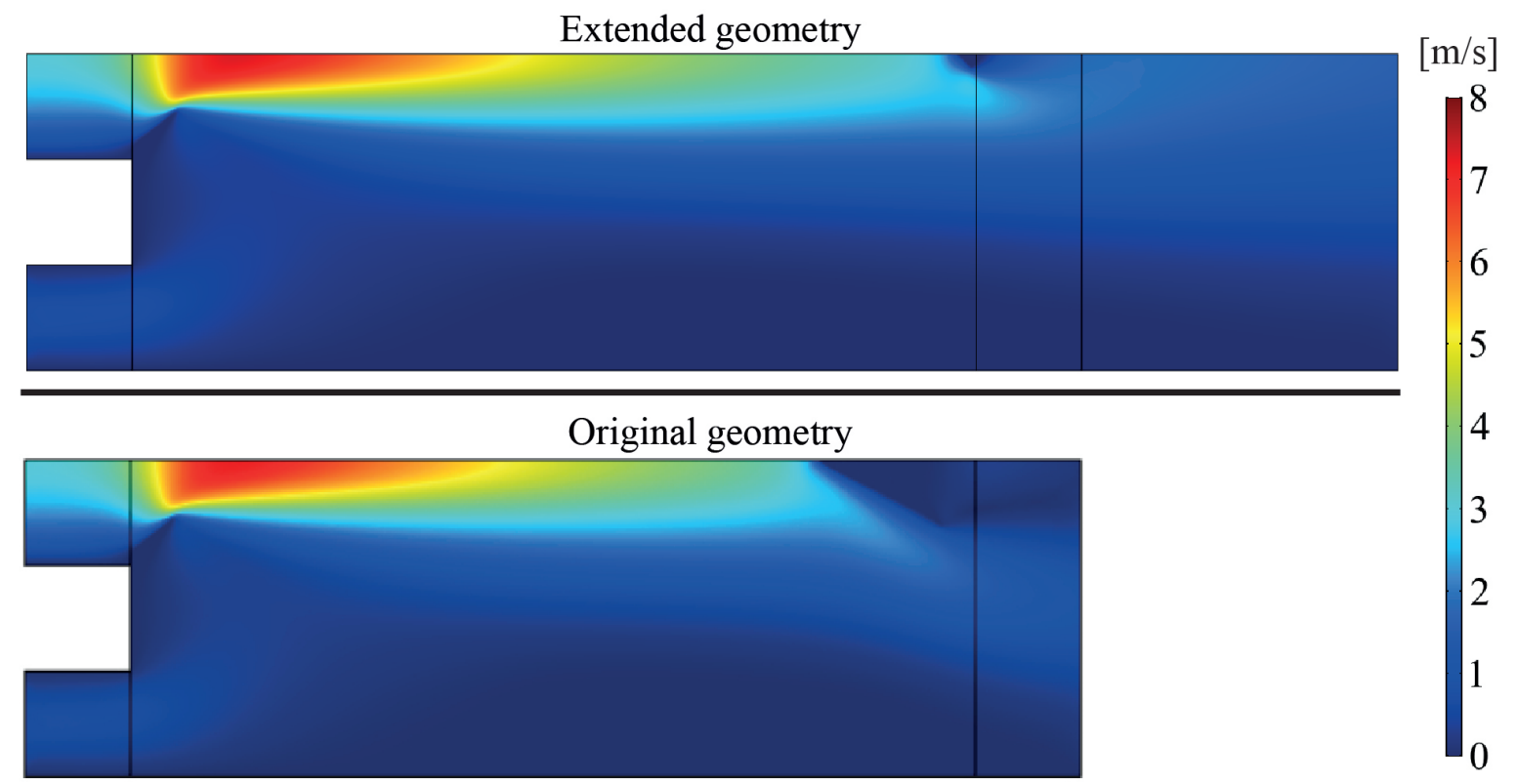

Source: Author. 
Figure 93 - Comparison between the pressure field of the original and extended geometries.

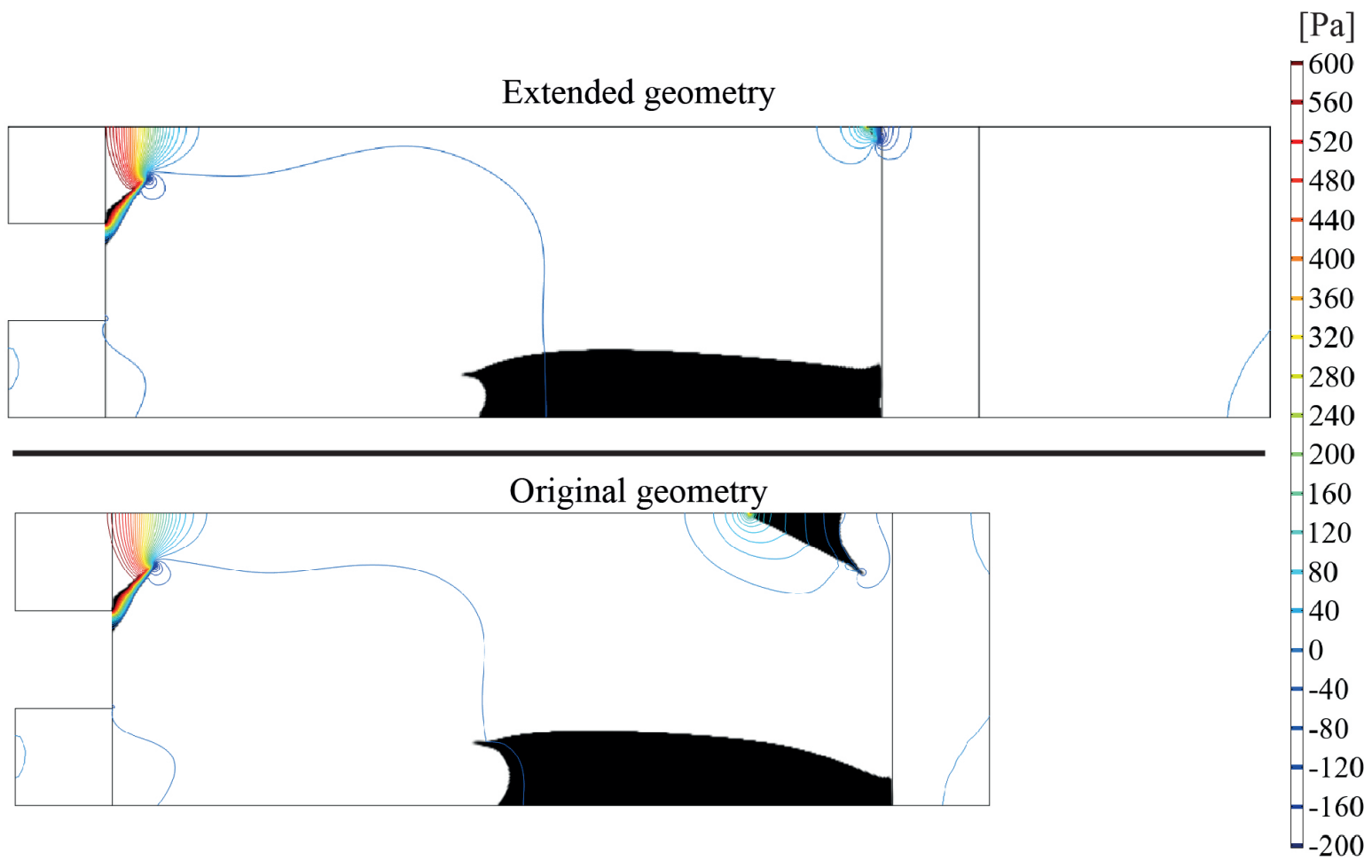

Source: Author.

Figure 94 - Comparison between the streamlines of the original and extended geometries.

Extended geometry

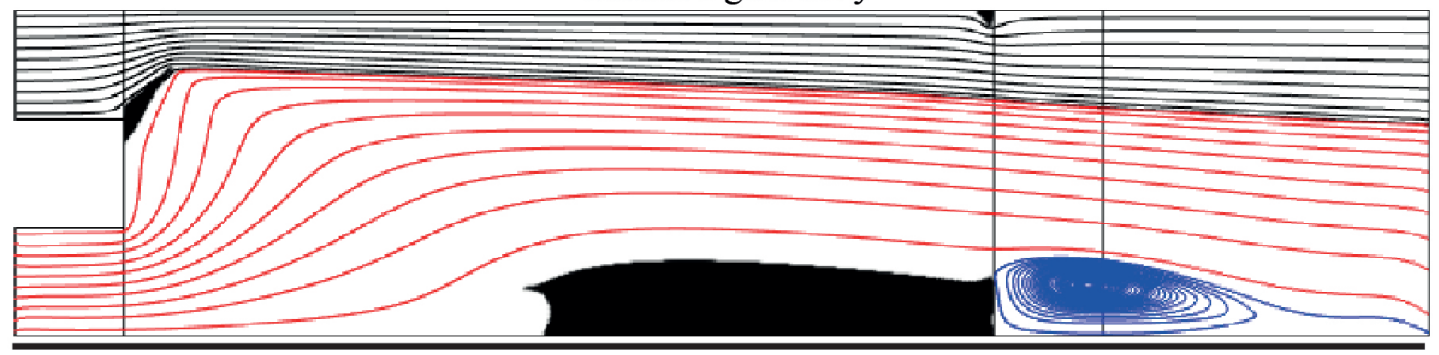

Original geometry

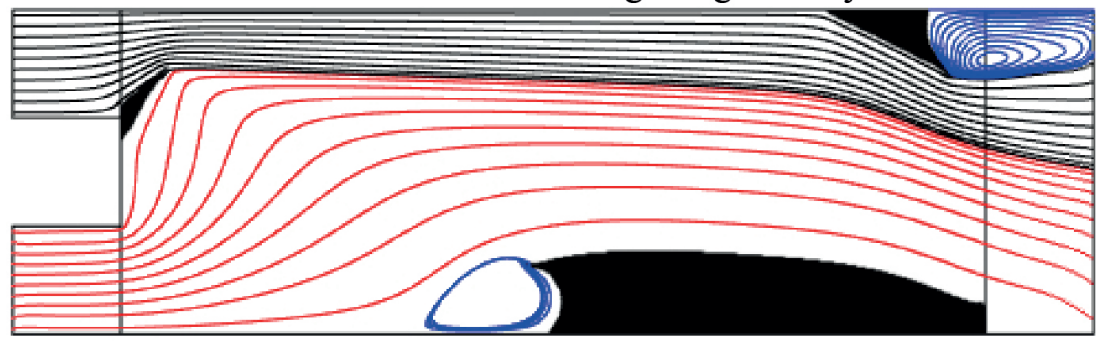

Source: Author. 